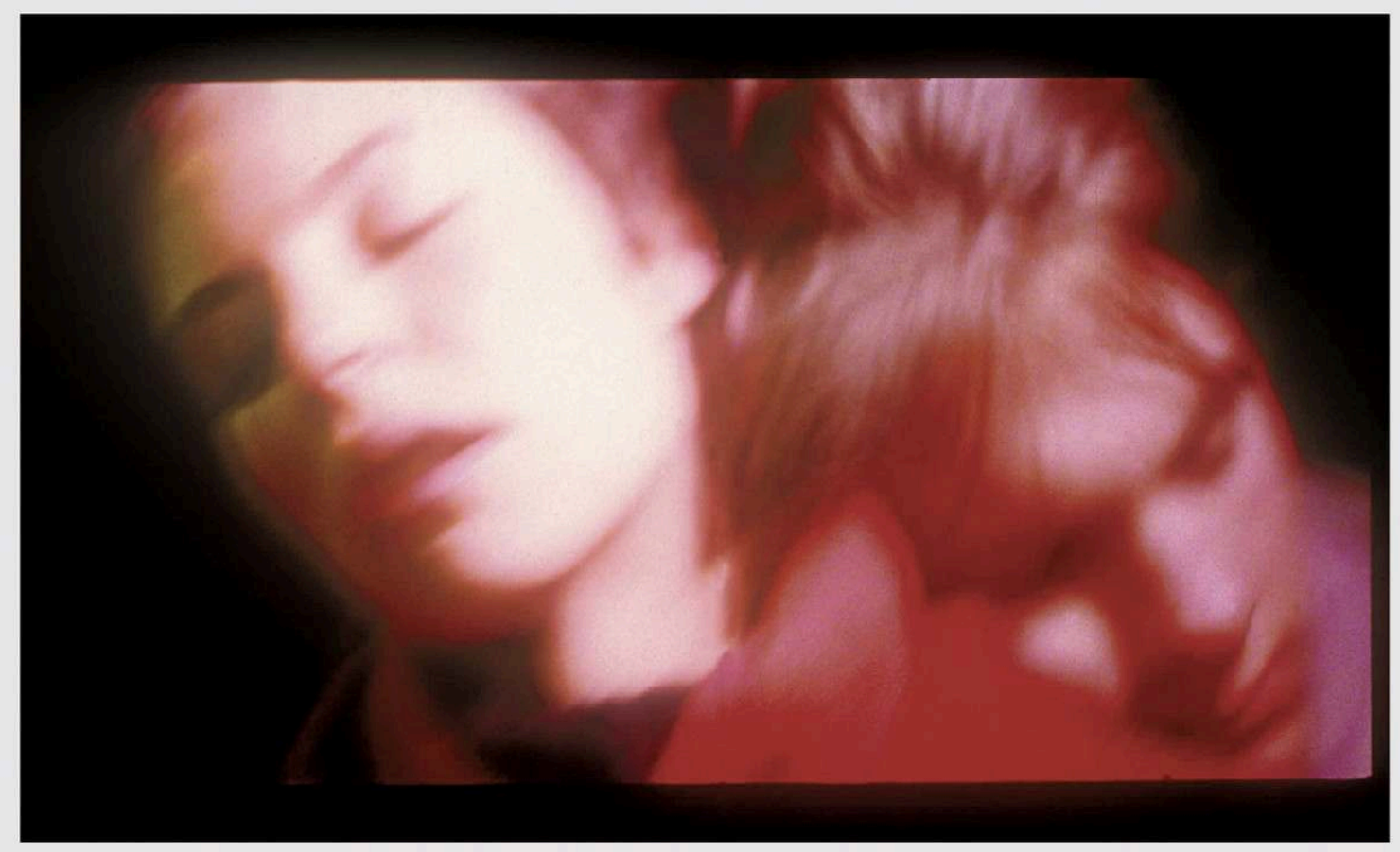

\title{
Images des corps / corps des images au cinéma
}

Sous la direction de Jérôme Game

ENS ÉDITIONS 


\section{Images des corps / Corps des images au cinéma}

\section{Jérôme Game (dir.)}

DOI : 10.4000/books.enseditions.9081

Éditeur: ENS Éditions

Lieu d'édition : Lyon

Année d'édition : 2010

Date de mise en ligne : 4 juillet 2019

Collection : Signes

EAN électronique : 9791036200489

\section{OpenEdition}

\section{Books}

https://books.openedition.org

\section{Édition imprimée}

Date de publication : 16 septembre 2010

EAN (Édition imprimée) : 9782847882124

Nombre de pages : 256

\section{Référence électronique}

GAME, Jérôme (dir.). Images des corps / Corps des images au cinéma. Nouvelle édition [en ligne]. Lyon : ENS Éditions, 2010 (généré le 28 janvier 2022). Disponible sur Internet : <http://books.openedition.org/ enseditions/9081>. ISBN : 9791036200489 . DOI : https://doi.org/10.4000/books.enseditions. 9081.

Légende de couverture

Illustration de couverture : photogramme extrait de La Vie nouvelle (2002), (C Philippe Grandrieux

(c) ENS Éditions, 2010

Conditions d'utilisation:

http://www.openedition.org/6540 


\section{RÉSUMÉS}

Ces dernières années ont vu se développer un important effort théorique et critique sur la question du corps au cinéma : comment ce dernier représente-t-il les formes du corps mais aussi ses puissances (gestes, désirs, pulsions, tendances hors cadre, hors champs, hors discours ou hors normes)? Comment rend-il compte des contextes cognitifs, culturels, politiques et technologiques associés à - ou affectés par - la fabrique des corps à travers l'histoire ? Les films eux-mêmes ont eu recours au corps comme figure ou motif privilégié et ont pleinement participé de ce questionnement. Contribuant à cette réflexion en cours le présent volume s'attache à étudier la question de la représentation corporelle en la rapportant à celle des devenirs concrets du cinéma, que ce soit dans ses dispositifs propres ou ses rencontres avec d'autres régimes d'image (notamment la vidéo et la photographie). L'ambition est en définitive d'offrir une sorte d'état des lieux de la question qui rayonnerait dans plusieurs directions : mise à l'épreuve des concepts ; articulation entre perspectives théoriques; poétique des oeuvres ; zoom sur certains points clés du rapport corps/image (la question de la vitesse notamment); enjeux culturels, politiques, esthétique et de genre ; question du récit, etc. C'est sur ces théâtres que se déploient les études réunies ici, chacune avec sa méthodologie et ses objets propres mais toutes envisageant la notion élargie de corps-image (au sens d'une matériologie générale du cinéma) comme possible paradigme critique.

JÉRÔME GAME (DIR.)

Maître de conférences en études cinématographiques et philosophie, Université américaine de Paris

\section{NOTE DE L'ÉDITEUR}

Illustration de couverture : photogramme extrait de La Vie nouvelle (2002), ( ) Philippe Grandrieux 
Cet ouvrage est diffusé en accès ouvert dans le cadre du projet OpenEdition Books Select.

Ce programme de financement participatif, coordonné par OpenEdition en partenariat avec Knowledge Unlatched et le consortium Couperin, permet aux bibliothèques de contribuer à la libération de contenus provenant d'éditeurs majeurs dans le domaine des sciences humaines et sociales.

La liste des bibliothèques ayant contribué financièrement à la libération de cet ouvrage se trouve ici :

https://www.openedition.org/22515.

This book is published open access as part of the OpenEdition Books Select project.

This crowdfunding program is coordinated by OpenEdition in partnership with Knowledge Unlatched and the French library consortium Couperin. Thanks to the initiative, libraries can contribute to unlatch content from key publishers in the Humanities and Social Sciences.

Discover all the libraries that helped to make this book available open access: https://www.openedition.org/22515?lang=en.

\section{IAI Sopenedition coupernoren}




\section{COLLECTION SIGNES}

dirigée par Éric Dayre 



\section{Images des corps / corps des images au cinéma}

Sous la direction de Jérôme Game

Avec les contributions de

Vincent Amiel, Martine Beugnet, Nicole Brenez, Sarah Cooper, Ludovic Cortade, Philippe Dubois, Jean-Michel Durafour, Elie During, Jérôme Game, Margara Millan, Pierre Sorlin, Emma Wilson 


\section{Éléments de catalogage avant publication}

Images des corps / corps des images au cinéma/ sous la direction de Jérôme Game ; avec les contributions de Vincent Amiel, Martine Beugnet, Nicole Brenez,... [et al.]. - Lyon : ENS Éditions, 20IO. - I vol. (256 p.) : couv. ill. ;22 cm. (Signes, ISSN I255-IOI5)

Notes bibliogr.

ISBN 978-2-84788-2I2-4 (br.) : 29 EUR

Tous droits de reproduction, de traduction et d'adaptation réservés pour tous pays. Toute représentation ou reproduction intégrale ou partielle faite par quelque procédé que ce soit, sans le consentement de l'éditeur, est illicite et constitue une contrefaçon. Les copies ou reproductions destinées à une utilisation collective sont interdites.

Illustration de couverture: photogramme extrait de La Vie nouvelle (2002),

(C) Philippe Grandrieux

(C) ENS ÉDITIONS, 2010

École normale supérieure de Lyon

I5 Parvis René Descartes

BP 7000

69342 Lyon cedex 07

ISBN 978-2-84788-2I2-4 


\section{Les auteurs}

Vincent Amiel, professeur d'études cinématographiques, Université de Caen

Martine BEUGNET, professeure d'études cinématographiques, Université d'Édimbourg Nicole BrEnEZ, maitre de conférences en études cinématographiques, Université Paris 1

Sarah CoOper, maître de conférences en études cinématographiques, Université King's College de Londres

Ludovic CORTADE, maître de conférences en études cinématographiques, New York University

Philippe Dubors, professeur d'études cinématographiques, Université Paris 3

Jean-Michel Durafour, chargé d'enseignement en études cinématographiques, Université Lille 3

Elie During, maître de conférences en philosophie, Université Paris 10 Nanterre Jérôme GAME, maître de conférences en études cinématographiques et philosophie, Université américaine de Paris

Margara Millan, professeure de sociologie, Université autonome de Mexico

Pierre Sorlin, professeur émérite, Université Paris 3

Emma Wilson, maitre de conférences en études cinématographiques, Université de Cambridge 
Ce livre a pour origine une journée d'étude sur les représentations cinématographiques du corps organisée le 6 avril 2007 à l'Université américaine de Paris sous la direction de Jérôme Game. L'ouvrage reprend les communications qui y furent présentées, réécrites et augmentées de quelques contributions.

Nous remercions tout spécialement Philippe Grandrieux d'avoir bien voulu nous autoriser à reproduire l'image en couverture, extraite de son film La Vie nouvelle (2002). 


\title{
Introduction Corps-cinéma
}

\begin{abstract}
Si la surface de notre très petit corps organisé (organisé précisément en vue de l'action immédiate) est le lieu de nos mouvements actuels, notre très grand corps inorganique est le lieu de nos actions éventuelles et théoriquement possibles: les centres perceptifs du cerveau étant les éclaireurs et les préparateurs de ces actions éventuelles et en dessinant intérieurement le plan, tout se passe comme si nos perceptions extérieures étaient construites par notre cerveau et projetées par lui dans l'espace. Mais la vérité est tout autre, et nous sommes réellement, quoique par des parties de nous-mêmes qui varient sans cesse et où ne siègent que des actions virtuelles, dans tout ce que nous percevons. Prenons les choses de ce biais, et nous ne dirons même plus de notre corps qu'il soit perdu dans l'immensité de l'univers.
\end{abstract}

H. Bergson, Les Deux Sources de la morale et de la religion.

Ces dernières années ont vu se développer un important effort théorique et critique sur la question du corps au cinéma, marqué notamment par l'ouvrage de Nicole Brenez, De la figure en général et du corps en particulier. L'invention figurative au cinéma, celui de Vincent Amiel, Le Corps au cinéma. Keaton, Bresson, Cassavetes, celui dirigé par Andrea Grunert, Le Corps filmé, ou encore celui de Steven Shaviro, The Cinematic Body.

I. N. Brenez, De la figure en général et du corps en particulier. L'invention figurative au cinéma, Bruxelles, De Boeck, I998; V. Amiel, Le Corps au cinéma. Keaton, Bresson, Cassavetes, Paris, PUF, 1998; A. Grunert, Le Corps filmé, Paris, CinémAction-Corlet, 2006; S. Shaviro, The Cinematic Body, Minneapolis, University of Minnesota Press, 1993. Tout récemment encore, R. Bellour revient sur ce thème dans Le Corps du cinéma. Hypnoses, émotions, animalités, Paris, POL, 2009, en étudiant le corps du spectateur pris dans celui 
Comment le cinéma est-il à même de représenter les facultés créatrices du corps, mais aussi ses puissances et ses formes irréductibles à toute typologie : gestes, désirs, pulsions, tendances hors cadre, hors champ, hors discours ou hors normes ?2 Comment peut-il rendre compte des contextes et instances cognitifs, culturels, politiques et technologiques associés à - ou affectés par - la fabrique des corps à travers l'histoire ?3 Ces questions essentielles, et les champs de recherche qu'elles recoupent, ont été bien entamées par les études récentes. Parallèlement, les films eux-mêmes, comme toujours, ont eu recours au corps comme topos, figure ou motif privilégié, et ont pleinement participé de ce questionnement.

Travaillant avec et sur ces différents discours ${ }^{4}$, le présent volume ${ }^{5}$ voudrait cerner une question précise: quel lien existe-t-il entre, d'une part, le cinéma compris comme dispositif de production/projection/perception

du cinéma. Voir aussi le dossier "Corps \& Cinéma», dirigé par P. Taliercio, Cadrage, mai-juin 2003, [en ligne], [URL: http://www.cadrage.net/dossier/corps_cinema/ corps_cinema.html], consulté le 2I février 20IO, ainsi que celui sur "La décomposition de l'humain. Le cinéma comme anthropologie virtuelle », dirigé par N. Rousseau dans la même revue en mars-avril 2002, [en ligne], [URL: http://www.cadrage.net/dossier/ anthropologie/anthropologievirtuelle.html], consulté le 2I février 2010.

2. Cette "peau fantasmatique de notre imaginaire corporel» que, "comme pellicule, il enveloppe", selon les termes de B. Andrieu, dans sa préface à E. Bayon, Le Cinéma obscène, Paris, L'Harmattan, 2007 , p. I7.

3. D'autant plus que, comme l'écrit C. Jaquet, «contrairement aux objets matériels, qui peuvent être appréhendés comme pure extériorité, le corps humain est un être complexe, car il brouille la distinction entre sujet et objet» (Le Corps, Paris, PUF, 200I, p. 7). Voir également C. Funtz éd., Les imaginaires du corps en mutation. Du corps enchanté au corps en chantier, Paris, L'Harmattan, 2008.

4. Pour ce faire, il convient de garder à l'esprit l'avertissement de M. Bernard dans son livre Le Corps: "Qu'on le veuille ou non, en effet, toute interrogation du corps s'opère toujours, directement ou indirectement, à l'intérieur du discours, tout comme ce discours luimême ne s'élabore que sur et par la résolution des processus matériels de la dynamique énergétique de nos pulsions et de leur projection fantasmatique. Il ne faut jamais perdre de vue que tout questionnement sur la réalité et la valeur du corps implique la reconnaissance en même temps que d'un certain statut corporel de ce questionnement lui-même, la discursivité inévitable du corps senti, conçu et interrogé. Questionnement, par conséquent, essentiellement inconfortable et périlleux dans la mesure où il ne faut jamais céder au mirage de la transcendance quasi immatérielle et neutre de l'omnipotence d'une parole scientifique maîtrisant un objet corporel, ni à l'illusion tout aussi séduisante de l'immanence matérielle d'un corps pulsionnel, réfractaire ou imperméable à toute verbalisation et donc à toute objectivation » (M. Bernard, Le Corps, Paris, Seuil, 1995, p. I63). Le cinéma, son écran, les images (de corps) qui y prennent corps constituent justement ici un objet complexe - à la fois construit comme dispositif et donné comme apparente «nature»à même de traduire ce double écueil dont parle Bernard, en quelque sorte de le figurer lui-même, pour lui donner le moins de prise possible et ainsi relancer l'étude.

5. Il est issu pour partie d'une journée d'étude que j'organisai à l'Université américaine de Paris le 6 avril 2007. 
d'un artefact plastique - écriture, mise en scène, cinématographie, montage, son, dispositif de projection, concourant tous, au final, à l'avènement d'une image et à sa perception physique -, et, d'autre part, l'informe natura naturans, la force dés-organ-isée/sante qu'est un corps - filmé, filmant ou regardant. ${ }^{6}$ En d'autres termes, il s'agit d'étudier ici, dans la voie notamment ouverte par les travaux déjà cités et ceux de Jacques Aumont, de Dominique Païni ou encore de Philippe Dubois ${ }^{8}$, le corps de l'image tout autant, sinon plus, que l'image du corps, et ce en vue de tester l'hypothèse d'une image-force faite de rapports intensifs, existant et opérant aux parages, ou dans, ou encore contre une image-forme, travaillant quant à elle l'objet.9 Et ainsi de participer à la réflexion en cours sur la question de la représentation corporelle au cinéma en la rapportant à celle des devenirs concrets de ce dernier, que ce soit dans ses dispositifs propres ou ses rencontres avec d'autres régimes d'image (notamment la vidéo et la photographie). L'ambition de ce volume est alors d'envisager la notion de corps-image comme paradigme critique rapportant directement la fabrique des corps et celle des images l'une à l'autre, faisant du récent propos de Jean-Marie Schaeffer - « $[\ldots]$ nous pouvons et devons dire indifféremment que notre pensée du corps est une pensée de l'image et que notre pensée de l'image est une pensée du corps ${ }^{10}-$ le principe d'une matériologie générale du cinéma.

«De quoi est-il donc question quand on parle de corps?» se demande Jean-Marie Brohm dans un article riche et synthétique intitulé « Le corps,

6. À cet égard c'est bien le corps pris dans la dialectique avec sa propre forme, et non la chair directement informe, qui est étudié ici. Voir B. Andrieu, «La chair sans image?», La Chair à l'image, S. Bernas et J. Dakhlia éd., Paris, L'Harmattan, 2006, p. 209-218. Voir aussi P. Weber, Le Corps à l'épreuve de l'installation-projection, Paris, L'Harmattan, 2003.

7. L'Invention de la figure humaine. Le cinéma, l'humain et l'inhumain, J. Aumont éd., Paris, Cinémathèque française (Conférences du Collège d'histoire de l'art cinématographique), I998, et J. Aumont, Matière d'images, Paris, Images modernes Cinéma, 2005 ; Projections, les transports de l'image, D. Païni éd., Paris, Hazan - Le Fresnoy - AFAA, 1997.

8. Rhétoriques du corps, P. Dubois et Y. Winkin éd., Bruxelles, De Boeck, I988; Cinéma et art contemporain III, P. Dubois éd., $\mathrm{n}^{\circ} \mathrm{I2}$ de Cinema \& Cie, 2009; et P. Dubois, «La question du figural», Cinéma. Art(s) plastique(s), P. Taminiaux et C. Murcia éd., Paris, L'Harmattan, 2004, p. 5I-76.

9. P. Vauday a traité un sujet similaire, d'un point de vue esthétique à la fois plus général dans son corpus et plus générique dans son approche: La Matière des images. Poétique et esthétique, Paris, L'Harmattan, 200I.

IO. J.-M. Schaeffer, «La chair est image», S. Breton éd., Qu'est-ce qu'un corps?, Paris, Musée du Quai Branly - Flammarion, 2006, p. 58-8I et particulièrement p. 62. 
un référent philosophique introuvable?»: «[...] quel corps? Il semble bien que le corps soit essentiellement un point de vue, un découpage de la réalité, car à la limite tout est corps et rien ne l'est. ${ }^{\mathrm{II}}$ De cette remarque générale se détachent deux paradigmes théoriques et critiques dans la période récente. Le plus connu est sans doute celui, phénoménologique, développé par Maurice Merleau-Ponty: le corps comme structure chiasmatique rapportant le monde externe au monde interne, ellipse entre

le dedans et le dehors, entre la chair et le monde (la chair dans le monde, le monde fait chair), entre la subjectivité et l'objectivité, entre le signifié et le signifiant, le conscient et l'inconscient. En tant que réalité sensible, chair, le corps est à la fois touché et touchant, senti et sentant, vu et voyant, même si le corps est fondamentalement le pivot de toute perspective sur le monde naturel ou socio-culturel, donc point de vue insurpassable et indépassé. ( $I b i d$.)

Le second paradigme est celui de Bergson. Contre tout dualisme, fûtil chiasmatique, Bergson pense le corps directement comme image. Dans l'océan infini des images pures qu'est le cosmos, l'une est particulière:

$[\ldots]$ en ceci qu'elle n'est pas seulement connue du dehors par des perceptions ou des sensations, mais aussi du dedans par des affections : le corps est une image vécue. [...] par elle, j'éprouve d'une part la dialectique de la variabilité et de l'invariabilité phénoménales, d'autre part la dialectique de la centralité et de la périphérie. (p. I39)

Qui plus est, le corps bergsonien n'est pas seulement une image dans l'espace, il l'est aussi dans le temps. Bergson écrit:

$[\ldots]$ nous pouvons parler du corps comme d'une limite mouvante entre l'avenir et le passé, comme d'une pointe mobile que notre passé pousserait incessamment dans notre avenir $[\ldots]$, replacé dans le temps qui s'écoule, il est toujours situé au point précis où mon passé vient expirer dans une action. ${ }^{12}$

Ainsi, dans ce second moment théorique, le corps-image (c'est-à-dire l'image comme corps plutôt que du corps) se caractérise par son immanente matérialité, fondamentalement ouverte, instable: en un mot, son devenir. Bergson de nouveau:

Voici un système d'images que j'appelle ma perception de l'univers, et qui se bouleverse de fond en comble pour des variations légères d'une cer-

I I. J.-M. Brohm, «Le corps, un référent philosophique introuvable?», Corps, $\mathrm{n}^{\text {os }}$ I2-I3 de Prétentaine, mars 2000, p. I29-I56 et particulièrement p. I4I.

I2. H. Bergson, Matière et mémoire, Paris, PUF, I939, p. 82-83. 
taine image privilégiée, mon corps. Cette image occupe le centre; sur elle se règlent toutes les autres; à chacun de ses mouvements tout change, comme si l'on avait tourné un kaléidoscope. (Ibid., p.20)

La centralité du corps-image n'est donc jamais fixe ni même statique: dans le flux incessant des images constitutif de la réalité, le corps-image est comme un pôle sans cesse effrangé, reconstitué sur et par ses marges. Les images alentour le déterminent en lui communiquant du mouvement, et vice versa:

Mon corps, objet destiné à mouvoir des objets, est donc un centre d'action [...]. [Les objets] renvoient donc à mon corps, comme ferait un miroir, son influence éventuelle; ils s'ordonnent selon les puissances croissantes ou décroissantes de mon corps. Les objets qui entourent mon corps réfléchissent l'action possible de mon corps sur eux [...]. Toutes les images se règlent sur une image centrale, notre corps, dont elles suivent les variations. ${ }^{13}$

Autrement dit, au sens le plus littéral qui soit, c'est avec mon corps que je vois, c'est lui qui regarde - autant qu'il est vu.

Ces deux conceptualisations du corps - celles de Bergson et de MerleauPonty - se doublent de deux façons d'approcher le cinéma. Pour Bergson, c'est l'«appareil» (de projection) qui origine le mouvement et le confère aux images: il est la machine abstraite ou virtuelle où se forme et d'où provient le «mouvement en général», «anonyme». Mais cette production est immédiatement réduite au statut d' «artifice» plus ou moins stérile et cantonnée à la pensée pratique ou celle qui a lieu «en général»: «[...] le mécanisme de notre connaissance usuelle est de nature cinématographique $»^{I 4}$, bien loin, ainsi, de notre capacité à percevoir "pour percevoir», bien loin de l'«accident heureux» - l'aptitude à créer - par où des «hommes surgissent dont les sens ou la conscience sont moins adhérents à la vie ». Is Bergson refuse au cinéma ce «détachement» qu'il consent pourtant aux «différents arts»-sculpture, poésie, musique, énumèret-il - et qui offre paradoxalement une «vision plus directe de la réalité». Puisque «c'est parce que l'artiste songe moins à utiliser sa perception qu'il perçoit un plus grand nombre de choses» (ibid.), le cinéma, à ce titre selon Bergson, est exclu.

I3. Ibid., p. I4-I5 et 2I, cité par J.-M. Brohm, «Le corps, un référent philosophique introuvable?" ", art. cité, p. I4O.

I4. H. Bergson, Euvres. L'Évolution créatrice, Paris, PUF, 1959 (Édition du centenaire), p. 753 .

I 5. H. Bergson, Euvres. La Pensée et le mouvant, Paris, PUF, 1959 (Édition du centenaire), p. 1373. 
Pour Merleau-Ponty, il s'agira d'étudier le cinéma sous l'angle de la psychologie de la perception : «[...] c'est par la perception que nous pouvons comprendre la signification du cinéma: le film ne se pense pas, il se perçoit.» Plutôt qu'une somme d'images, il est une structure qui nous donne directement la forme d'une «conduite» ou d'un «comportement», cette «manière spéciale d'être au monde, de traiter les choses et les autres, qui est pour nous visible dans les gestes, le regard, la mimique, et qui définit avec évidence chaque personne que nous connaissons $» .{ }^{16}$ Identifiant l'opération cinématographique à la signification philosophique du spectacle produit (la sensation qu'on en a) plutôt qu'à sa genèse technique, Merleau-Ponty en extrait la leçon: ce que le cinéma-psychologie nous donne et que la "philosophie phénoménologique ou existentielle» tente de décrire, c'est le "paradoxe» de la «conscience jetée dans le monde». Cette conscience, on peut la nommer conscience-corps. L'expérience d'un film devient ainsi niveau transcendantal à partir duquel saisir la nature chiasmatique de l'être: «[...] le cinéma est particulièrement apte à faire paraitre l'union de l'esprit et du corps, de l'esprit et du monde et l'expression de l'un dans l'autre» (ibid.). Et Merleau-Ponty d'insister sur cette identification: «le mélange de la conscience avec le monde, son engagement dans un corps, sa coexistence avec les autres », ce sujet-là «est cinématographique par excellence» (p.75). ${ }^{17}$

Dans son traité sur le cinéma - formé de L'Image-mouvement et de L'Image-temps - Gilles Deleuze se souviendra de ces deux monuments théoriques. En assonance avec Merleau-Ponty, il soutiendra que la formefilm n'est pas réductible à la somme de ses parties (visuelles ou sonores) en ce qu'elle génère un saut qualitatif qui révèle la nature profonde de toute image : «Le cinéma ne nous donne pas une image à laquelle il ajouterait du mouvement, il nous donne immédiatement une image-mouvement. $\gg^{18}$ «[C]oupe mobile» (ibid., p. 87), l'image-mouvement actualise sans cesse les paradoxes du mouvement et du tout par rapport au fixe et aux parties. Cependant, en lecteur assidu des stoïciens, Deleuze ne conçoit pas les images comme des copies de choses ou d'êtres mais bien comme les choses elles-mêmes. À strictement parler, les images sont dès lors pour lui toutes

I6. M. Merleau-Ponty, «Le cinéma et la nouvelle psychologie», Senset non-sens, Paris, Gallimard, 1996 [1966], p. 6I-75 et particulièrement p.74.

I7. Voir C. Zernik, " "Un film ne se pense pas, il se perçoit." Merleau-Ponty et la perception cinématographique », Rue Descartes, n 53 , 2006, p. IO2-IO9.

I8. G. Deleuze, L'Image-mouvement. Cinéma I, Paris, Minuit, I983, p. II. 
les choses du monde. Ce matérialisme radical de l'image - et la profuse taxonomie à laquelle il donnera lieu dans les deux volumes de Cinémaest en réalité profondément bergsonien, fût-ce contre ce que Bergson luimême avait soutenu du cinéma : l'identité corps / images l'emporte, dans ses implications, sur l'apparent dualisme réel / image-mouvement. C'est en fait cette dernière, explique Deleuze, qui confère toute sa portée à la manière dont Bergson a dépassé le dualisme matière/esprit. ${ }^{19}$ Maurizio Grande écrit:

Chez Deleuze l'image-mouvement est la réalité («ce qui apparaît»); l'image cinématographique l'est au même titre, étant donné que les objets sont des images, et que les qualités, les perceptions et les actions ne sont que des images «spécialisées» qui se forment dans l'interaction et la modification continue des images-corps. [...] on peut constater que chez Deleuze l'image-mouvement est à la fois la "matière-image» (le réel) et l'imagematière (le cinéma), et que les aspects de la «matière-image» donnent lieu aux différents genres et types de l'image cinématographique. [...] Cela revient à dire qu'on ne peut pas tracer une ligne de séparation entre la réalité et l'image-mouvement, et que le «réel» est une question de degrés de la matière et des «coupes» qu'on appelle qualités, perceptions, actions, et qui présentent les variations et les entrelacements de l'image-mouvement dans le "plan d'immanence» (de la matière et du cinéma). ${ }^{20}$

Dans le chapitre viII de L'Image-temps intitulé «Cinéma, corps et cerveau, pensée ${ }^{21}$, Deleuze fait passer sa grande idée : la pensée est comme ce qui arrive au corps depuis la surface où les devenirs se forment et ont lieu, agençant la matière depuis elle-même dans un temps a-chronologique. Et réciproquement: le corps est ce qui force à penser depuis la matière, à la faveur d'un geste ou d'une posture, à même les choses plutôt que par-dessus elles. Les dichotomies du dualisme s'estompent, inutiles - sujet/objet, interne / externe, donneur d'ordre / instrument, pensant / pensé (non pensant). Le renversement du platonisme, grand dessein deleuzien, est affaire de corps et de surfaces paradoxales (voir le mot de Valéry souvent cité par Deleuze: «le plus profond, c'est la peau»). Renversement

I9. Sur les rapports entre Deleuze et Bergson au sujet du cinéma, voir A. Sauvagnargues, «L’image. Deleuze, Bergson et le cinéma», L’Image, A. Schnell éd., Paris, Vrin, 2007, p. $157-176$.

20. M. Grande, «Les images non dérivées», Le Cinéma selon Deleuze, O. Fahle et L. Engell éd., Paris, Presses de la Sorbonne-Nouvelle, 1997, p. 284-302 et particulièrement p. 285-287. Sur la réaffirmation du bergsonisme par Deleuze autour de la question du cinéma, voir aussi, dans ce même ouvrage, J.-P. Esquenazi, «Deleuze et la théorie du point de vue. La question du signe », p. 372-387.

2I. G. Deleuze, L'Image-temps. Cinéma 2, Paris, Minuit, 1985. 
du cartésianisme aussi bien, car corrélativement, l'image de la pensée se transforme: elle n'est plus vue comme œuvre ou procès d'un sujet-source, cohérent et projeté ou rétrospectif, mais comme affect / signe d'un «cogito dissous », d'un "je fềlé » insistant dans l'Aiôn - c'est-à-dire d'un corps se (re-)faisant.

Jacques Rancière est celui qui a le plus précisément pointé les difficultés possiblement tautologiques de cette théorie du point de vue d'une étude du cinéma comme art. Si Deleuze a raison, soutient Rancière, alors "cinéma n'est pas le nom d'un art. C'est le nom du monde».22 Si les images existent purement en soi, au titre de la matière même, sans avoir à être constituées pour être (sans avoir à être cadrées notamment, ou agencées dans leur mouvement par le montage), comment parler du cinéma sans risquer le solipsisme?

Le point de départ de Deleuze n'était-il pas [que la perception] a toujours été [dans les choses], que ce sont les choses qui perçoivent, qui sont en rapport à l'infini les unes avec les autres? La définition du montage apparait alors paradoxale : le montage donne aux images, aux événements de la matière-lumière, des propriétés qui leur appartiennent déjà. (Ibid., p. I49 $)^{23}$

De même concernant l'aptitude qu'aurait le cinéma à fabuler le réel tout en restant fidèle à son ouverture fondamentale, Rancière énumère les objections. Commentant le rôle et les puissances que Jean Epstein assigne au cinéma, il résume tout d'abord la façon dont Deleuze en a été profondément influencé:

La vie ne connait pas d'histoire. Elle ne connait pas d'actions orientées vers des fins, mais seulement des situations ouvertes dans toutes les directions. Elle ne connait pas de progressions dramatiques mais un mouvement long, continu, fait d'une infinité de micro-mouvements. [...] L'automatisme cinématographique règle la querelle de la technique et de l'art en changeant le statut même du «réel». Il ne reproduit pas les choses telles qu'elles s'offrent au regard. Il les enregistre telles que l'œil humain ne les voit pas, telles qu'elles viennent à l'être, à l'état d'ondes et de vibrations, avant leur qualification comme objets, personnes ou événements identifiables par leurs propriétés descriptives ou narratives. [...] Ce que l'œil mécanique voit et transcrit, nous dit Epstein, c'est une matière égale

22. J. Rancière, La Fable cinématographique, Paris, Seuil, $200 \mathrm{I}$, p. I48.

23. Nous soulignons. C'est d'ailleurs là une ligne critique que Rancière porte à l'ensemble de l'esthétique deleuzienne: voir "Existe-t-il une esthétique deleuzienne?", Gilles Deleuze. Une vie philosophique, E. Alliez éd., Paris, Synthélabo - Les Empêcheurs de penser en rond, 1998 , p. 525-536. 
à l'esprit, une matière sensible immatérielle, faite d'ondes et de corpuscules. Celle-ci abolit toute opposition entre les apparences trompeuses et la réalité substantielle. [...] L'écriture du mouvement par la lumière ramène la matière fictionnelle à la matière sensible. [...] Tel est le drame nouveau qui a trouvé avec le cinéma son artiste. (p.8-9)

Rapportant ensuite ce projet moléculaire à celui, littéraire, de Flaubert - qu'il englobe dans ce qu'il nomme le «régime esthétique des arts » ${ }^{24}$-, Rancière précise :

C'étaient bien, en un sens, des "plans de cinéma » que cadraient les micronarrations flaubertiennes en nous présentant Emma à sa fenêtre, absorbée par la contemplation de ses échalas de haricots renversés par la pluie, ou Charles accoudé à une autre fenêtre, le regard perdu sur la paresse d'un soir d'été, les quenouilles des teinturiers et l'eau sale d'un bras de rivière industriel. (p.I6-I7)

Mais c'est pour mieux nous rappeler une spécificité technique essentielle au cinéma, vite noyée selon lui dans les ellipses epsteino-deleuziennes, et qui pourtant l'institue en véritable rupture: la caméra ne peut pas se rendre elle-même passive, n'a pas les moyens d'un devenir-passif apte à saisir la passivité des corps auxquels un devenir survient, et ce pour une raison simple, c'est qu'elle est déjà, et de toutes origines, passive elle-même; c'est une machine et non une âme, un cogito ou un sujet.

Les cadrages flaubertiens, c'était le travail de l'écriture qui contredisait par la fixité rêveuse du tableau les attentes et les vraisemblances narratives. Le peintre ou le romancier se faisaient les instruments de leur devenir-passif. Le dispositif machinique, lui, supprime le travail actif de ce devenir-passif. La caméra ne peut pas se faire passive. Elle l'est de toute façon. Elle est nécessairement au service de l'intelligence qui la manipule. (p. I7)

Pour saisir la passivité des corps et leur indétermination, le cinéma devrait donc d'abord se contraindre à une maîtrise technique mais ensuite immédiatement la contrarier, afin de s'empêcher de filmer en rond. Réfléchir au cinéma des corps est-ce alors autre chose que réfléchir au cinéma tout court, à son art(efactualité) comme à ses puissances? En tout état de cause, les différentes tensions possiblement aporétiques contenues dans et entre les paradigmes rapidement évoqués ci-dessus nous semblent utiles en ce qu'elles constituent - avec les films eux-mêmes - l'un des théâtres d'opération pour une intervention critique aujourd'hui, en permettant

24. Voir Jacques Rancière. Politique de l'esthétique, J. Game et A. Wald Lasowski éd., Paris, Archives contemporaines, 2009. 
une sorte d'état des lieux de la question qui rayonnerait dans plusieurs directions: mise à l'épreuve des concepts; articulation entre perspectives théoriques; poétique des œuvres; zoom sur certains points clés du rapport corps/image (la question de la vitesse notamment); enjeux culturels, politiques, esthétiques et de genre; question du récit; etc. C'est sur ces terrains que se déploient les études réunies ici, chacune avec sa méthodologie et ses objets propres.

La première partie du livre s'intitule «Flux » et s'attache à saisir le lien entre le corps comme mouvement et l'image cinématographique comme production de flux. Que ce soit sous la figure du rhizome chez Yoshida Kijū (Jean-Michel Durafour), du vecteur chez Hou Hsiao-hsien, Ozu et Wong Kar-wai (Vincent Amiel) ou encore de l'informe chez Philippe Grandrieux, Arnaud des Pallières, Douglas Gordon et Philippe Parreno (Martine Beugnet), il s'agit de cerner comment les corps - et les personnages avec eux se décomposent et recomposent dans le mouvement et ses perceptions. La deuxième partie, intitulée "Virtuel», interroge la fabrique technologique des corps autour de différents régimes de vitesse: du ralenti chez Jean Epstein, Dziga Vertov et les frères Wachowski (Elie During) à la modulation (accélération/ralenti) entre photo, cinéma et installation vidéo chez Jean-Louis Gonnet, Harmut Lerch, Klaus Holtz, Thierry Kuntzel, Emmanuel Carlier et Egbert Mittlestädt (Philippe Dubois). En une troisième partie titrée "Récits", ce sont les portées éthique, géographique et narrative d'une reconfiguration de l'économie corporelle par celle, filmique, du cinéma qui sont envisagées chez les frères Dardenne (Sarah Cooper), Bruno Dumont (Ludovic Cortade), Carlos Reygadas et Gus Van Sant (Jérôme Game). "Politiques», la dernière partie, analyse enfin les puissances de redistribution du sens d'un cinéma des corps - par exhibition, complication ou occultation - au travers des figures de l'amour chez Philippe Garrel, Pedro Costa et Sharif Waked (Nicole Brenez), de la foule dans le cinéma de la première moitié du $\mathrm{Xx}^{\mathrm{e}}$ siècle (Pierre Sorlin), du genre chez Kimberly Peirce (Emma Wilson) et du métis chez Julian Hernandez et Carlos Reygadas (Margara Millan).

Les corpus filmographiques comme les enjeux cernés par ces études, ou encore leurs approches critiques, sont suffisamment variés pour offrir une perspective large sur le thème du corps au cinéma. Ce qui les rassemble est l'importance qu'elles confèrent à ce qui fait image dans le corps, comme à ce qui, dans l'image, prend corps. Leur pertinence tient notamment à ce qu'elles se saisissent avec précision et originalité de cette question depuis l'intérieur des films, plutôt qu'en les surplombant. Par-delà leur 
diversité, leurs résultats font apparaittre la continuité d'une double détermination propre au corps cinématographique : site d'inscription de forces diverses où se lisent les vulnérabilités comme les stratégies de résistance à l'ordre des discours, il est aussi ce qui ne cesse de reposer la question de ce à partir de et à travers quoi le mouvement sur l'écran, la productivité interne au film comme l'économie de son sens demeurent actifs et en jeu. C'est le nouage de ce double statut, de cette simultanéité intensive du corps au cinéma - qu'on pourrait appeler faute de mieux le rapport sens / puissance ou fond / forme ou encore sujet / devenir - qui fonde la matériologie générale du cinéma dans laquelle ce volume ambitionne de s'inscrire: pouvoir iconoclaste et reconfiguration du réel, telles sont les deux faces, intimement liées, du corps-image. 

Flux 



\section{::: \\ Des corps effacés par le flux : Hou Hsiao-hsien, Ozu et Wong Kar-wai}

Le visage de Shu Qui, souriant, se retourne vers la caméra, qui flotte doucement au rythme de la démarche chaloupée de la jeune femme. Lumières, musique, évanescence des gestes et de la voix : nous sommes, spectateurs, dans une réalité diffuse, relative, portés par un mouvement proprement inqualifiable, jouant sur le ralenti, la molle suspension de la steadycam, l'incongruité des lieux. La silhouette s'enfonce dans un tunnel, au bas d'escaliers descendus en sautillant, et le noir occupe l'écran. C'est l'ouverture de Millenium Mambo de Hou Hsiao-hsien (200I). Un corps happé par le mouvement, un corps-mouvement qui impose à l'image son rythme propre. Comme si le corps-personnage s'effaçait au profit d'un corpsimage portant le regard du spectateur et l'empêchant en définitive. Un corps qui ne serait plus au centre de l'image, mais qui serait son vecteur. Son vecteur et sa victime, puisqu'il disparait avec elle, entraîné par son propre mouvement. Dans la représentation classique, un objet ne disparaît pas avec son support de représentation: il ne lui est que contingent, persistant dans la mémoire, la transparence et la reproduction. Ce que Millenium Mambo met en place, c'est précisément la condition (la mise en scène) d'un corps dont l'image ne serait pas distincte, c'est-à-dire d'un corps qui produit le regard, dont le mouvement emporte celui-ci, et qui ne peut exister indépendamment de la perception qu'il procure. Le ralenti n'est pas seulement le ralenti de l'objet, mais celui du regard, préparant ces moments où le flux des images, affolant le regard, effacera les corps.

Ce premier plan du film de Hou Hsiao-hsien est matriciel, au sens où il fournit une position d'où tout le reste du film découle, aussi bien en termes de narration que de perception. Un moment dans le temps, libéré de tout contexte historique - on est en 2012, arbitrairement -, libéré donc 
d'attaches de quelque sorte qu'elles soient, et duquel les images qui suivent seront regardées; comme regardées "de ce corps», mobile, emporté par le flux.

Tout le film décline une opposition entre des corps flottants, soumis aux mouvements qui les portent (bousculades, danses, virées automobiles), et la vaine tentative d'arrêter ces courses. Tout le film traite de ces flux incessants et de la peur sporadique qu'ils génèrent, par éclats, comme des éclairs de conscience. La vie de l'héroïne est ainsi faite de mobilités successives, non raccordées, non préméditées, et qui ne peuvent composer une totalité, puisque aucune raison ne les ordonne. Sa seule conscience est perceptive : la vitesse dans la voiture, l'ivresse dans la boîte de nuit. Perceptions sans mémoire, donc sans possibilité d'ordonnancement rétrospectif. Le récit lui-même en est impossible: il se perd en de multiples sources, comme s'il était impossible d'en fixer un point de départ. La voix off ne correspond pas aux images, les chronologies sont dispersées, comme s'il n'y avait effectivement pas de centre possible à la narration. Ni un personnage au présent ni une conscience rétrospective: juste les lignes emmêlées de dérives aux régimes autonomes.

\section{Cristallisation}

Un seul moment échappe à ces dérives accumulées, deux scènes dans l'hiver d'une station de ski japonaise. Parenthèse diégétique, mais aussi et surtout sensible, pendant laquelle non seulement les courses s'arrêtent, mais se heurtent à une temporalité différente, comme une cristallisation soudaine, un moment où le corps se repense comme unité. La première scène se déroule dans la cuisine d'une vieille femme qui prépare une soupe de légumes: son obstination tranquille, la lenteur de ses gestes, la patience avec laquelle elle attend la fin de la cuisson obligent les corps à se ramasser, à se retrouver autour d'une sensation inusitée qui est aussi bien celle de l'attente que celle du fumet de légume. Rôle essentiel que celui des perceptions culinaires, goût et odorat, dans les films asiatiques. Ils ont chez Hou Hsiao-hsien une forte connotation nostalgique, mais aussi, paradoxalement, sont des marqueurs très nets du présent, et de la puissance d'identité que celui-ci impose. Il y a une incontestable vivacité de l'être-là dans les scènes de dégustation, ces scènes où l'on goûte, par la bouche ou par le nez, ce qui s'offre là, dans l'épaisseur de la durée que donne la sensation. Comme si le corps entier se mettait à exister autrement, autour de l'objet 
du goût, et cessait d'appartenir uniquement aux courants fugitifs. Rien ne passe par la psychologie des personnages: ce n'est pas en ce sens un épisode narratif qui se déroule dans la cuisine. C'est une autre image: une autre façon de suivre les gestes, de fermer le cadre. L'atmosphère est différente : les corps y sont confrontés à une autre expérience de l'instant, qui semble exister pour lui-même. Une identique épreuve de soi a lieu un peu plus tard, lorsque les personnages vont marcher dans la neige. Ils s'amusent à tomber dans les congères, et à y imprimer leurs visages. Le contact du froid soudain sur la peau, là encore, fonctionne comme un arrêt brutal; et ce moment dans la neige devient un moment unique, marqué dans le souvenir comme il est marqué dans le présent, parce que la sensation, tout à coup, fige la durée. C'est un leurre, aussi éphémère évidemment que l'empreinte du visage dans la neige; mais comme lui, il cristallise une forme qui arrête le continuum entropique. Le voyage au Japon marque par antithèse le destin de ces corps toujours enfuis: en signalant la possibilité d'une conscience fulgurante de soi par une sorte de coagulation momentanée des sensations, elle met en relief la perte ordinaire de cette identité.

\section{Ni corps ni personnages}

Ce que propose Millenium Mambo, après sa fantomatique ouverture, c'est en définitive, plus que la disparition d'un corps, l'inaptitude à en composer un. L'éclatement du récit d'une part, et la diversité des perceptions instructurées d'autre part conduisent à empêcher de fait que s'organisent avec assez de permanence les gestes et les comportements qui ordonnent l'idée d'un personnage, lui donnent logique et congruence. Et au-delà, ce sont les perceptions d'une entité corporelle qui ne correspondent plus les unes avec les autres. Ce n'est pas seulement la silhouette qui se perd à l'écran dans la foule des rues ou des bars, qui disparait dans les nuits successives, c'est la conscience de soi, et plus encore la conscience de son propre corps. Ou plus exactement l'expérience de ce que Merleau-Ponty appelle «la chair», c'est-à-dire non pas une "conscience de » mais une «conscience d'être», une épreuve de soi. Dépossédée de son corps, l'héroïne du film l'est en termes diégétiques, épiée par son amant, traquée jusque dans les odeurs de sa peau, dans le plus intime de sa vie physique, mais elle l'est aussi en termes existentiels: ce n'est pas tant qu'elle ne s'appartienne plus, c'est qu'elle ne se constitue plus. La suite des mouvements qui la portent ne lui offre pas une continuité d'être, elle brouille au contraire la possibilité d'un lien identitaire. 
Il est intéressant sur ce plan de rapprocher Millenium Mambo du cinéma français des années 1920 , avec lequel le cinéma asiatique contemporain a tant à voir sur le plan formel. Dans un film comme La Glace à trois faces de Jean Epstein (1927), où la vitesse tient une place prépondérante, le personnage principal est l'objet d'expériences physiques extrêmes dues à la vitesse de son automobile. Cette vitesse, provoquée, recherchée, en affectant sa sensibilité, constitue son personnage. C'est un homme «construit» par la vitesse, et les différentes scènes, elles-mêmes architecturées par un montage singulier, s'organisent pour donner au spectateur un équivalent sensible de ce qu'éprouve le conducteur. Le personnage et ses perceptions sont ainsi les objets du film qu'ils organisent eux-mêmes. Montage rapide, effets de filage, mouvements vertigineux vont dans le même sens, d'une corporéité qui s'affirme à la mesure des expériences qui l'affectent. On pourrait dire la même chose de bien des films de l'avantgarde française des années 1920. Cela met d'autant plus en valeur l'évanescence dans laquelle Hou Hsiao-hsien maintient son personnage, tout juste suggéré, ébauché à grands traits pour mieux être effacé par le déroulement du film.

Formidable paradoxe cinématographique, au terme duquel on peut affirmer que plus on voit le personnage, moins il existe... Il y a quelque chose du New Rose Hotel d'Abel Ferrara (1998) dans ce retournement brillant, mais qui précisément ne regarderait jamais du côté de la performance ou du trompe-l'œil séduisant. Le vertige lui-même donne à sentir un corps dans son rapport au monde: or il n'y a pas de vertige dans Millenium Mambo. Juste des éclats, des bribes, des trajectoires sans aboutissement.

Hou Hsiao-hsien entreprend d'une certaine manière la démonstration de ce principe de disparition dans Three Times (2005), qui met en regard non seulement "trois temps», mais aussi trois histoires, et trois corps. Le titre du film corrobore le lien étroit entre ces corps et le temps dans lequel ils sont enfermés; que ce temps soit continu ou discontinu, la durée perçue ou non. L'héroïne de l'un des épisodes ( Le temps des amours») vit dans un temps que l'on pourrait qualifier de "proustien », mêlant les sensations, les retrouvant ou croyant les retrouver d'une époque à l'autre, construisant autour d'elle un système de perception aux balises précises, aux cadres stables, à l'intérieur duquel la nostalgie peut faire son œuvre. En contrepoint, l'un des deux autres épisodes («Le temps de la jeunesse ») retrouve la mise en scène de Millenium Mambo, et son temps sans armatures, pour corps qui s'effondrent. Là encore, le film s'ouvre sur la fluidité du mouvement, pris ici dans la trame de tant d'autres, croisés, 
suivis, perdus, oubliés, les flux ininterrompus et multiples des voitures dans la circulation périurbaine. Là encore, la caméra n'épouse que relativement le trajet du corps emporté de l'héroïne: elle a parfois la même allure qu'elle, entraînée à la même vitesse dans les mêmes courbes, et parfois elle prend son autonomie, change de tunnel, se laisse distancer. Ce corps dont nous éprouvons parfois les sensations, et qui devient parfois objet du regard peine alors, ici aussi, à se constituer comme unité. Il n'appartient à aucune histoire - toutes les séquences suivantes en embrouillent la possibilité -, à aucun des personnages - éclatés en facettes inconciliables -, à aucune perception.

À trois reprises le trajet dans la ville réactive cette sensation, et les flux en faisceau entraînent chaque fois la potentialité d'une dérive nouvelle. Chaque fois, effectivement, alors que les trajets semblent relativement semblables, les péripéties sur lesquelles ils ouvrent sont inattendues, mettant en scène des lieux nouveaux, des rencontres improbables. La répétition du cadrage pouvait créer dans d'autres circonstances - on y reviendra - une unité de perception, une continuité temporelle, elle irrite au contraire, ici, toute velléité d'unité - celle-ci fût-elle progressive. Avec cet épisode de Three Times, Hou Hsiao-hsien fait littéralement éclater l'équivalence entre répétition et continuité, la permanence des formes s'associant systématiquement à la dispersion des temps et des perceptions. Davantage qu'un paradoxe, c'est, en termes de narration, une disjonction particulièrement iconoclaste (au sens figuré comme au sens propre: une destruction de l'image comme signe, comme information persistante); la répétition des formes n'y est plus gage de pérennité. Les plans des jeunes personnages pénétrant dans la circulation de Taipei, se laissant glisser en moto dans son mouvement ininterrompu, ces plans répétés ne donnent aucune information temporelle, n'indiquent ni durée ni constance, ils sont au contraire les figures d'un temps happé par le mouvement.

Jing, la jeune héroïne du «Temps de la jeunesse», l'épisode moderne de Three Times, se trouve à plusieurs reprises confrontée à des situations de perte de soi. Des photographies lui sont comme «arrachées» lors d'un récital, pendant lequel l'objectif du photographe la cerne, vient la toucher presque, la saisir pendant qu'elle chante; sa vie, ses mouvements, son corps sont, dans l'instant de leurs pulsations les plus fiévreuses, arrêtés par la photographie, détournés, soumis à un autre régime. Comme ses crises d'épilepsie, régulièrement, la privent de la fluidité et de la continuité de ses mouvements. Ces deux façons de n'être plus soi - de n'être plus son corps -, de se voir dépossédée de fragments, de lambeaux arrachés 
au continuum pour devenir des entités, se rejoignent dans l'incapacité de tenir un corps. Jing qui suit les mouvements de la ville, qui danse à ses rythmes, n'y existe pas en tant que corps. Ou plutôt, si elle y existe, de sensations en sensations, c'est dans une dépossession permanente, le contraire d'une expérience aux résultats accumulés et aux inflexions résultantes. Corps tiraillé par les rencontres, corps enlevé de soi; corps filant, comme une étoile qui s'efface dans sa propre course.

Les personnages modernes de Hou Hsiao-hsien sont ainsi toujours à la limite de ne pas en être, à peine cristallisés en tant que tels, entraînés par leur corps vers la déliquescence. Par le mouvement de leur corps à l'image. Non seulement, donc, ceux-ci ne sont pas les supports quelconques d'une entité scénaristique transcendante, qui les dépasse et les asservit, mais ils empêchent que cette entité ne se constitue, ils en perdent la potentialité par leur figurabilité même. Apparence obligée de la période classique, représentation minimale parce que nécessaire d'un personnage qui lui préexiste, le corps cinématographique devient, dans ces films emportés par la vitesse, la cause première de l'extinction des unités individuelles: psychologiques, sociales, existentielles.

\section{Yasujiro Ozu, premier maître}

Dans les premiers films de Hou Hsiao-hsien, comme dans l'épisode «Le temps des amours» de Three Times, revient cette figure de mise en scène qui consiste à répéter un cadrage sur un espace singulier, et à y faire évoluer des personnages dans des situations différentes. On repère ces dispositifs dans Un été chez Grand-Père (1984), ou dans Poussière dans le vent (1986), par exemple, où les gares de banlieues sont les espaces de passage, tantôt grouillants, tantôt déserts, des voyageurs auxquels se mêle le couple principal. Dans La Fille du Nil (1987), c'est le seuil d'une maison: toujours un lieu de passage, et les occurrences différentes en fonction des visiteurs. Ce sont des cadres dans lesquels le temps se replie: où la répétition des angles de vision entraîne une réminiscence quasi automatique, partagée, selon les cas, par le spectateur et les personnages. Dans Three Times, la salle de billard occupe cette fonction, espace proustien dans lequel le corps se souvient, c'est-à-dire où la sensation accompagne la reconnaissance. Dans ces types de scènes, évidemment, le cadre vide prend une importance, et un intérêt, qu'il n'aurait pas autrement: il existe par le lien que la répétition instaure, et il désigne autant le vide que toutes 
ses alternatives possibles. Ici, face à l'absence, le corps se prend à exister au gré des souvenirs, des rêves, des possibles imaginés. Il existe parce qu'il a existé, ou qu'il pourrait exister. Dans le cadrage immuable de la salle de billard ou du quai de la petite gare de banlieue, c'est tout sauf le vide, ou l'abstrait, qui s'impose : c'est l'afflux irrationnel et simultané des corps possibles, des moments d'occupation, des manières d'habiter cet espace. Là se cristallise une identité par l'écho même des occurrences dispersées. Là s'identifie une sensibilité: sans cette unité, ni mélancolie possible, ni projets, ni palpitation du temps.

$\mathrm{Ce}$ «levier dramatique», cette forme singulière de la répétition / fixation, par le biais du cadrage, viennent sans doute, en droite ligne, du cinéma d'Ozu. Hou Hsiao-hsien a souvent dit l'admiration qui est la sienne pour le cinéaste japonais. À vrai dire, rares sont les cinéastes asiatiques qui n’ont pas, d'une façon ou d'une autre, une dette envers cet immense créateur. Mais ce qui, en l'occurrence, est particulièrement remarquable, c'est leur capacité commune à fixer le temps et le mouvement comme peu d'autres cinéastes, et de faire habiter cet espace-temps par des corps vivants.

Ce sont, chez $\mathrm{Ozu}$, des couloirs vides aux lignes géométriques où passent des silhouettes, des surcadrages de fenêtres ou d'encadrements de portes, des façades uniformes d'où se détachent des bustes immobiles. La grande question que posent ces films est une quête de sagesse : «Comment habiter le monde? » Comment l'habiter et comment s'en détacher, puisque aussi bien il faudra le faire? Le rapport des corps à l'espace de l'image n'est pas chez Ozu un jeu formel, il est le rapport du sujet au temps.

Un couple sur une terrasse couverte, au début de Voyage à Tokyo (1953): ils préparent leurs bagages, se penchent ensemble, se redressent ensemble, leurs têtes pivotent en même temps. Un peu plus tard, chez leurs enfants, le mari et la femme sont de profil, et agitent leurs éventails au même rythme. À la fin du film, veuf, le mari est sur la terrasse du début, face au même paysage; il agite doucement l'éventail. Rien ne marque l'absence, parce que rien n'a jamais été marqué: les mouvements sont aériens, les gestes ne laissent pas de trace. Ce sont, à proprement parler, des évanouissements. Chaque instant s'évanouit, s'est toujours évanoui : on ne trouvera pas une inscription qui déclenche la mélancolie, sur un cahier ou sur un arbre, on ne trouvera pas un objet patiné par les manipulations qui rappellerait une attitude, une main posée cent fois. Non, au geste qui s'est perdu succède un geste qui s'est perdu. C'est la leçon de ces vides, qui empêchent de se laisser prendre dans les plis du temps, d'habiter des coins ou des resserres du monde où les souvenirs peuvent devenir insupportables. Les chorégraphies 
des personnages d'Ozu sont détachées de la palpitation du moment: elles se déroulent sur fond de ciel, de mer, de murs nus. Elles dés-habitent le monde, elles s'en détachent en s'accomplissant. Il manque un geste à côté de soi, une autre main qui agite un éventail, mais rien n'est tenté pour arrêter ce geste. Si les corps chez Ozu hantent les cadres comme des fantômes, c'est qu'ils en sont déjà - toujours - partis. Au loin la grand-mère joue avec son petit-fils: ils occupent cette ligne aérienne que sont les chemins herbeux dans tous ces films. Leurs silhouettes se détachent, comme les cheminées, les enseignes, les objets qui balisent l'espace d'Ozu. Mais la caméra se rapproche, et l'on entend la femme évoquer l'avenir, et le temps où son petit-fils sera devenu médecin : «Et moi, que serai-je devenue alors? » La silhouette au loin, essentielle au cadre, n'est déjà plus là, en quelque sorte, et rien ne gardera sa trace, sur l'horizon infiniment ouvert.

Les cadres fixent les corps, mais les espaces qu'ils circonscrivent sont en même temps traversés d'une vie autonome, d'un souffle qui balaie ce qui pourrait malheureusement y rester attaché. Ce qui unit au-delà de tout le vieux couple et sa bru, c'est la manière qu'elle a de s'agenouiller avec eux, de saluer en même temps qu'eux, de mêler la courbure de son buste aux leurs, de s'associer pendant quelques scènes à leurs balancements communs. Plus qu'un geste, précis, qu'un acte digne de souvenir, davantage que tous les mots prononcés, c'est l'instant, quelques secondes, d'un mouvement du corps sans intention qui marque le lien. Indéfectible, parce qu'évanescent, volatil dans la permanence des encadrements. Autant de paradoxes qui mettent en avant le rôle primordial du corps, à la fois inscrit à l'image et dans le monde, et produisant ces mouvements infimes, fugaces, détachés de tout contrôle et de toute volonté. Cultiver ces gestes (c'est-à-dire en permettre la production aussi bien qu'en saisir le moment), c'est s'assurer d'une vie qui ne pèsera pas, d'une existence qui ne pourra pas produire de passé. Il n’y a chez Ozu que du temps qui passe; jamais de temps passé: parce que les corps qu'il filme sont dans le moment de l'effectuation, en même temps qu'ils semblent associés à la permanence des choses. C'est le paradoxe même de l'image mouvante, c'est le grand écart stupéfiant que réalise ce cinéma, fixant le temps - des natures mortes - et trouvant dans la matière même de sa figuration le ressort de l'évanouissement - le geste pur.

«Passons, passons, puisque tout passe. Je me retournerai souvent $»^{\mathrm{I}}$ : le beau vers d'Apollinaire est inadéquat ici, et c'est en cela que ce cinéma

I. G. Apollinaire, «Cors de chasse», Alcools [19I3], Euvres complètes, Gallimard (la Pléiade), Paris, 1965 , p. I 48. 
est une leçon de vie. Le temps de se retourner, et tout est à nouveau identique, le geste accompli, perdu dans le vent, et il reste, imperturbablement, le cadre de la porte, le balustre, le coin de rue. Le corps n'a pas marqué, n'a pesé en rien sur les choses; détaché du monde, il peut en accepter la perte avec plus de légèreté.

Ce détour par le cinéma d'Ozu est essentiel, je crois, pour comprendre ce qui se joue aujourd'hui chez Hou Hsiao-hsien. Parce que l'espace de ses films est, lui, hanté par la présence de corps qui laissent leur empreinte. De corps qui habitent le cadre: un père, une grand-mère, une hôtesse de bar. Il reste des lettres, des souvenirs sensibles, une empreinte dans la neige, fût-ce quelques secondes... Il reste - au moins dans les films de la première partie de l'œuvre, où les figures spatiales se répètent - trop de choses dans le cadre.

D'où peut-être ce changement de registre, qui fait des corps, fébrilement, les éléments d'un flux qui, d'une autre manière, les efface. Disparition encore, évanouissement, mais qui entraine avec lui l'évanouissement du monde. Comme s'il fallait qu'en se mêlant à ces courses, à ces trajets sans fin, les personnages abandonnent à leur corps le sort de leur identité et de leur existence. Les jeunes hérö̈nes de Hou Hsiao-hsien emportent dans leurs courses la condition même de l'épreuve, le réseau de sensations qui leur donne une conscience d'être. L'image de Millenium Mambo et du «Temps de la jeunesse» dans Three Times est image d'un corps en relation. Comme si, consubstantiellement, celui-ci se déplaçait avec le cadre, avec sa matière, couleurs, lumières, objets, avec l'épaisseur de sensations possibles dont il est constitué. Ainsi s'éloignent et réapparaissent, toujours les mêmes et toujours différents, les jeunes amants sur leur moto, dans le flux de la circulation. Ils ne vont nulle part et ne viennent de nulle part - contrairement aux personnages d'Ozu; mais ils nourrissent leur existence des rapports constants de leur corps à l'image. Ils sont, interminablement, et ne sont que, cette image.

\section{Sur la route d'Iguazu}

Plus violemment, plus radicalement - sans passer par la stabilité possible du cadre -, le cinéma de Wong Kar-wai accomplit cette fusion du corps et du mouvement - ou peut-être serait-ce plus juste de dire qu'il la prend en compte, et la décline de film en film. Chez Wong Kar-wai une absence d'ancrage est au principe de tout: ni lieu ni temps ne peuvent constituer 
le contexte stable d'un état, d'un souvenir, d'une représentation. Toute pensée y est fuyante, mobile, emportant les corps au-delà d'une forme situable, pour leur substituer une trajectoire. C'est l'histoire de presque tous ses films, de Chunking Express(1994) aux Cendres du temps(I994), et de Happy Together (1997) à 2046 (2004). Dans ses deux dernières œuvres en particulier, qui forment un réseau mouvant dans lequel flottent des corps sans identité, les images acquièrent vis-à-vis des repères diégétiques une autonomie étonnante et rare. Que ce soient les ralentis hypnotiques de Maggie Cheung et Tony Leung allant acheter leurs nouilles sous la pluie, ou le montage répété de gestes se détachant de toute subjectivité, les images de In the Mood for Love (2000) sont à ce titre exemplaires. Le récit les a abandonnées, comme livrées à leur propre mouvement, à leur chorégraphie triste et rituelle; ce pourraient être des souvenirs mélancoliques; ce pourraient être de timides et délicates rencontres; ce pourraient être des fantasmes nés d'une imagination musicale... Ce sont avant tout les dérives de corps qui ne valent que par leurs mouvements, c'està-dire par leurs rythmes, leurs rimes, les échos de leurs déplacements, tout ce dont les images sont elles-mêmes constituées. Un léger recul de la femme, une course sous la pluie, une main sur une autre, vite enlevée, autant d'éléments qui seraient autant de signes dans une narration classique, et qui se perdent ici dans l'indécision des identités pour former la matière sensible d'un monde où le geste et le moment se cristallisent dans la couleur et la lumière des choses.

Incarnée, la durée l'est, pourrait-on dire, par la rencontre du geste et de la texture de l'image dans laquelle il se déploie, qui est la texture du monde par lequel il se comprend lui-même. Les rideaux de pluie, les trottoirs luisants, la lumière rouge au travers des rideaux, l'étroitesse des portes de l'appartement commun, cette présence de la sensation physique, de l'épaisseur des choses, marque les corps d'une curieuse empreinte, un vade-mecum turbulent, qui participe au chaos des assemblages. Si, chez Ozu, les corps pouvaient s'évanouir de n'avoir pas adhéré aux aspérités du monde, ici ils s'évanouissent d'y avoir échangé trop intimement des sensations toujours différentes. Hong Kong, la Thaillande, le Cambodge, une année, un mois, un séjour, une odeur de soupe d'hiver, un plat de printemps, une musique de Nat King Cole, une valse triste, un air chinois: tout contexte est marquant, précis, tranchant, dans In the Mood for Love, et à aucun moment ces totalités autonomes ne se fondent en une trajectoire. Dans Happy Together, les sautes culturelles et sensitives sont encore plus marquées, et la voix off ne suffit pas - c'est l'un des intérêts du film - à en effacer le disparate. Ainsi, 
sautes ou répétitions ont le même effet, produisent la même dissémination vertigineuse de moments. Mais il ne s'agit pas seulement d'une question d'ordonnancement narratif ou de montage. Il ne s'agit pas d'une opération rétrospective, et qui viendrait surplomber les épisodes vécus : il s'agit bien au contraire de moments présents déconnectés dès le déroulement d'une unité plus large que pourtant ils opèrent. On est au cœur du système de Wong Kar-wai: si le corps ne se souvient pas de son passé, ou se coupe, pour une raison ou pour une autre, de ce qui pourrait le relier à une expérience vécue, c'est que, dans le présent même, sont étouffées les potentialités de continuité. Comme chez Alain Resnais, l'immédiat de la perception l'empêche par nature d'établir des histoires qui soient autre chose que des inventions. Dans In the Mood for Love, quand les gestes des personnages se veulent la répétition de ceux de leurs conjoints, ils créent ainsi de toutes pièces une histoire de ces gestes, mais se coupent alors radicalement de toute possibilité d'une histoire de leurs propres corps. Voilà pourquoi 2046 se présente avant tout comme une constellation d'images, qui sont toujours des moments du corps, mais que le récit ne peut en même temps convoquer dans leur puissance et résorber dans une continuité. On inverse précisément le constat de Wim Wenders notant qu'au cinéma «l'histoire suce le sang des images $»^{2}$; ici, les images ne sont plus que chair et sang, ce qui paradoxalement empêche tout corps de se constituer.

2046 et In the Mood for Love, au plus proche des corps et des gestes, désignent la limite où ces corps, emmenés par leur propre mouvement, désorganisent leur rapport au monde, c'est-à-dire en définitive disparaissent comme entités derrière ce rapport éclaté en une multitude d'occurrences. Les corps n'y sont plus que mouvements, désirs, échappées, puissances, jusqu'à l'ensevelissement. Se reproduit par exemple, dans l'un et l'autre film, la figure sidérante d'un personnage simulant l'action d'un autre, ou répétant la sienne à un autre moment. Figure qui cristallise ici le pli serré des désirs et du présent du corps. Dans le moment où le geste est fait, il est traversé de désir; dans le moment où le mot est prononcé - d'approche, de rupture, de passion - le corps accompagne sa dynamique.3 Que geste ou déclaration n'appartiennent pas au personnage à ce moment-là, puisqu'il

2. W. Wenders, La Logique des images. Essais et entretiens, M. Töteberg éd., traduction B. Eisenschitz, Paris, L'Arche, 1990.

3. Ce n'est pas vrai par principe : on connait dans les récits classiques cette scène du personnage répétant à l'avance l'aveu gênant qu'il va produire; la situation crée généralement d'abord une confusion, puis donne au contraire la mesure de la conscience qui régit un tel simulacre. Dans le film de Wong Kar-wai, rien de tel. 
les mime ou les reproduit, met précisément en valeur, par l'imposture du corps, l'absence de ce dernier comme unité. Seul existe le mouvement, celui du désir, de la honte, du déchirement. Ces mouvements s'accumulent comme des strates, plutôt que s'organiser en une figure éclatée; ajoutés les uns aux autres, ils laissent les corps à leur destin d'enveloppes vides.

Dans Happy Together, ce ne sont plus des strates fines aux infimes décalages, mais des courants fulgurants et des mouvements de houle profonds qui défont les corps - ou qui, plutôt, empêchent qu'ils ne se constituent. Là aussi, la matière de l'image dissémine: noir et blanc, couleurs, sépia, ralentis, accélérés perturbent l'unité de réception et de perception. Mais plus que cet éclatement des régimes d'images, c'est la puissance de leurs flux qui marque dans Happy Together le devenir des corps. Le dernier tiers du film, en particulier, reprenant sous d'autres formes les divers errements auxquels le personnage a été soumis, multiplie les brusques échappées, les épreuves sans lien d'un corps soumis à des contraintes hétéroclites. "D'une certaine manière, il s'agit toujours de saisir le fragment alors qu'il va s'évanouir et se dissoudre dans une prolifération désordonnée", écrivait Noël Herpe à la sortie du film. ${ }^{4}$ Or ce fragment qui s'évanouit, c'est d'abord l'expérience sensible que le corps éprouve d'une condition donnée. Ainsi se succèdent les lents travaux dans un abattoir, au contact répétitif des quartiers de viande et du sang qui se répand, puis les flux agités de la circulation à Buenos Aires, avant que ne se suspende la durée, le temps d'un trajet vers les chutes d'Iguazu. En une série de petits clips dont les musiques très typées rendent les coupures encore plus nettes, la dispersion des élans et des mouvements se donne à sentir à la mesure des lieux précisément mentionnés. Là encore, c'est l'affirmation des couleurs, des textures, des vitesses, qui, conjuguée au mouvement constant des corps, efface la possibilité d'un contour net de la trajectoire. Tout est tressautement, et en définitive tout est fantomatique; tout est embrasement, et tout est consumation. Dans une des rares séquences où la musique de tango semble raccorder des moments disparates et des gestes aux contextes différents, le corps qui danse finit son mouvement au ralenti, épuisant sa chorégraphie sur un trottoir de petit matin, et s'abandonne à l'immobilité comme à une disparition. Visuellement, dramatiquement, les corps s'effacent en effet d'être soumis à tant de forces divergentes, et à ces régimes d'images qui décentrent constamment la source de leur perception.

4. N. Herpe, «Happy Together. Je ne peins pas l'être, je peins le passage», Positif, $\mathrm{n}^{\circ} 442$, décembre 1997, p. 6. 
Après avoir ainsi ballotté son corps d'océan en océan, de trajet en trajet, de hurlements en mutisme, le personnage de Tony Leung rentre «chez lui»: dans un Hong Kong dont il parait avoir gardé les odeurs et le contact en souvenir, mais qui peut-être n'est qu'une expérience nouvelle de plus, l'ultime expérience de la vitesse. Après avoir erré entre les marchands de brochettes et de soupe - parfums du passé, sensations qui cadrent et arrêtent le temps -, et après n'y avoir rien trouvé qui le rende à lui-même, le voici happé par la déformation de la ville que lui propose la vitesse du métro aérien. À nouveau la fuite, le vertige de sens bouleversés qui entrainent avec eux le monde; les images filent, le corps disparaît. Heureux de nouveau, peut-être, de se déliter dans une autre succession d'impressions.

Comme Hou Hsiao-hsien, Wong Kar-wai impose aux corps autre chose qu'une présence ou une disparition diégétique: il leur donne une image nouvelle. Une image filée, qui ne fixe plus, qui ne "prend» plus; un assemblage de visions qui ne se rapporte pas à un centre, ni à une expérience sensible - qui évite l'expérience de soi-même. Le flux de ces images ne peut entraîner que l'enveloppe des choses, comme seule l'enveloppe du corps accompagne les déflagrations de la perception. La chair, la sensation de soi, les corps ramassés et consistants sont restés dans la stabilité des cadres lisses d'Ozu. 



\section{:::}

\section{D’un corps chez Yoshida Kijū. Le cinéma: du mouvement au déclenchement}

Le cinéma est l'art du mouvement: on l'a dit et redit, l'affaire semble entendue, comme si le mouvement à l'écran allait de soi sous prétexte que le mobile a effectivement existé devant la machine analogique. Mais ne pourrait-on pas plutôt y voir l'art du problème que constitue tout mouvoir (arrachement, commencement, engagement, effort, etc.)? L'art de ce qui pose problème et donc fait arrêt avant ou dans l'entame de tout mouvement, voire pour que le mouvement puisse tout simplement être? Je voudrais dans les pages qui suivent tester cette hypothèse : le mouvement sur l'écran fait migrer visuellement le problème mécanique et secret de son enregistrement (sa décomposition en photogrammes) comme de sa restitution (sa reconstitution pendant la projection). Il en constituerait ainsi comme une proposition esthétique éclairant ce qui, de tout corps en mouvement, butte dans le mouvement même, "au lieu de s'oublier dans la légèreté essentielle du sourire». ${ }^{\mathrm{T}}$

Où la question est ici abordée à partir de quelques propositions deleuziennes

Gilles Deleuze et Félix Guattari le rappelaient en un énoncé lapidaire : «Le réel flue. $»^{2}$ Une telle formule tire à l'eau de divers puits philosophiques et

I. E. Levinas, De l'existence à l'existant, Paris, Vrin, 2004, p.3I.

2. G. Deleuze et F. Guattari, L'Anti-CEdipe, Paris, Minuit (Critique), I972, p. 43. 
se tient à leur jonction nodale pour embrayer à partir de là. Au-delà du paradoxe, toujours reconduit, consistant à exprimer ce qui ne se fixe pas avec des mots (chosifiant), l'enjeu, qui traverse sous une forme ou une autre rien de moins que toute l'histoire de la pensée occidentale, est de taille: penser le réel sub specie durationis. C'est-à-dire le penser non pas comme un fait - sorte de ready-made primordial, self-ready-made pourrait-on dire - mais comme ce qui se fait, non pas comme ce qui est mais comme ce qui devient, non pas comme formes - détourées, murées mais comme forces - déroutées, expansives.

Dès son apparition, le Cinématographe, apte étymologiquement à «écrire le mouvement» - constat ou mot d'ordre? -, a participé à la composition du «catalogue des vecteurs de mouvement qui traversent le monde $\gg{ }^{3}$ Il n'avait en effet de prime abord qu'à poser l'appareil de prise de vue pour les enregistrer - en fait les morceler en autant de poses immobiles, ou photogrammes, dynamisées, tonifiées artificiellement a posteriori - sans faire, à ce stade, intervenir d'autre manipulation que le cadrage. Chez les frères Lumière et leurs opérateurs: arrivée ou départ d'un train ou d'un bateau, sortie d'usine, défilés et processions, bals, leçons de bicyclette, passants lyonnais, et ainsi de suite. On abordait là un chapitre supplémentaire de l'idée selon laquelle le réel n'a presque rien à voir avec la réalité mais presque tout avec la réalisation. L'objectif de la caméra devenait ainsi cet appareil, plus intègre que l'œil, plus impartial, permettant de mieux capter la «branloire perenne» du monde, là où notre vision ordinaire a tendance, au contraire, à substituer des choses aux actions, des états aux changements, des objets aux trajets pour mieux s'orienter, agir ou connaître. "Extérieur aux objets et invisible, le mouvement de l'appareil d'observation s'est reporté dans les figures des objets, où il est devenu visible comme une mobilité propre, animant chaque forme et lui permettant de se modifier. ${ }^{4}$

Le Cinématographe, et par la suite le cinéma, avec l'apparition des premières formes de montage en tant que tel ${ }^{5}$ - encore plus le cinéma comme

3. P.-A. Michaud, «L'apparition du réel. Le cinéma des Lumière», Sketches. Histoire de l'art, cinéma, Paris, Kargo, 2006, p. 73.

4. J. Epstein, «Le monde fluide de l'écran» [1950], Écrits sur le cinéma, t. II, Paris, Seghers (Cinémaclub), 1975, p. I48.

5. Nous entendons par là le montage immanent au film. Il existait dès les premières projections de vues ou de films courts une sorte de montage extrinsèque opéré par le conférencier qui présentait les vues, choisissait l'ordre de leur passage, regroupait certains films en série et liait oralement les films les uns aux autres. 
on va le voir ${ }^{6}-$, devaient donc finir par rencontrer la philosophie des devenirs. Le point culminant, théoriquement parlant, de cette rencontre en serait, peut-être, la description proposée par Deleuze dans Cinéma I de la perception engagée par le film comme «perception aquatique». Quel en est le principe? Notre vision commune est organisée pour l'existence terrestre. Nous percevons sur terre (ou, certes, parfois ailleurs - mer, air mais comme sur terre: bateau, avion, etc.), et cette perception possède trois caractéristiques précises: I / la subjectivité (la perception est perception d'un je conscient); 2 / la transcendance (ce je entretient avec ce qu'il perçoit des rapports d'extériorité sujet/objet); 3/l'unicentrement (la perception part du $j e$, centrifuge, puis y revient, centripète). Le cinéma va liquéfierliquider cette perception, en nous plongeant in medias res, nous donnant accès, non à de nouveaux objets à voir, mais à une nouvelle manière de voir les objets. Soit désormais: objectivité, immanence, acentrement. La perception n'est plus rapportée à un je conscient extérieur aux choses comme un terminus a quo et ad quem. Avec la mobilité de la caméra et le montage, avec la variabilité des points de vue et des échelles de plan, le cinéma fait quitter l'immobile dans lequel nous aménageons d'ordinaire - en cherchant, à partir de lui, à «guetter le mouvement au passage»-, et nous permet de «nous replacer dans le mouvant pour traverser avec lui les positions immobiles $\gg .^{7}$ Or cet état de la perception, poursuit Deleuze, est exactement celui que l'on trouve, non pas sur terre donc, mais sur l'eau. «Sur terre, le mouvement se fait toujours d'un point à un autre, est toujours entre deux points, tandis que, sur l'eau, c'est le point qui est entre deux mouvements. $»^{8}$ Autrement dit, si la perception terrestre fonctionne par points fixes - privilégiant l'arrêt sur le mouvement, tenant le mouvement pour un moment provisoire entre deux arrêts -, sur l'eau, sur la mer par exemple, il y a «perte vertigineuse des points fixes» (ibid.) - là où la terre est ferme, fermée - et tous les points cartographiques se valent. De fait, «l'eau est le milieu par excellence où l'on peut extraire le mouvement de la chose mue, ou la mobilité du mouvement lui-même» (p. II2). C'est précisément ce que fera le cinéma, par le montage ou les mouvements

6. Le Cinématographe était encore par trop statique : prises de vues généralement fixes, un plan par scène ou simple bout-à-bout, narration souvent théâtrale, caméra trop lourde et peu maniable. Les expérimentations visuelles existaient mais sont restées isolées jusque vers la fin de la première décennie du $\mathrm{xx}^{\mathrm{e}}$ siècle.

7. Pour ces deux citations, H. Bergson, «Introduction à la métaphysique » [I903], La Pensée et le mouvant, PUF (Quadrige), I998, IO édition, p.2I2.

8. G. Deleuze, L'image-mouvement. Cinéma I, Paris, Minuit (Critique), I983, p. II5. 
d'appareil: donner à voir le mouvement en «le dégageant de son enveloppe sensible", à condition de «le dissocier de ce corps qu'il traverse, qui le porte et auquel il ne s'identifie pas».9

\section{Le cinéma: jaillissement rhizomatique entre les corps}

Que trouve-t-on dans le réel? Mille plateaux apporte une première réponse: d'un côté, des «espaces lisses ", proprement définis par la «nomadologie », et, de l'autre, des "espaces striés », sédentaires. ${ }^{10}$ Si les espaces striés ne s'intéressent qu'aux formes et aux substances (extensives) en introduisant des arrêts et des poses dans les mouvements du monde, les espaces lisses en laissent intacts les flots: singularités et intensités. Si les premiers sont les plus courants, eu égard à notre perception terrestre usuelle, dont on vient de rappeler qu'elle ne cesse d'endiguer l'universel déferlement de la matière (sous forme d'arrêts et de choses), les seconds composent le courant même du réel, qui n'est que fugue.

Dans les espaces lisses, qui sont comme une "enfilade de portes $»^{\text {II }}$, des corps se baignent, barbotent, nagent, crawlent, flottent, dérivent; sont attirés, sont repoussés; désirent. Ces corps, il faut donc les distinguer de ceux qui habitent les espaces striés, hiérarchisés et polarisés; il faut les différencier des organismes. Les organismes sont constitués d'organes clos, formés et nettement distincts les uns des autres, qui ne communiquent entre eux que sous certaines conditions (de subordination notamment) et en conservant leur identité métrique. Les corps sans organes, à l'opposé, puisqu'il s'agit d'eux, détricotent l'organisme et sont non différenciés, incessamment ouverts à «des connexions qui supposent tout un agencement de circuits, des conjonctions, des étagements et des seuils, des passages et des distributions d'intensité, des territoires et des déterritorialisations mesurées à la manière d'un arpenteur» (p. 198). Ils ne sont que des « usines à gaz », «ensemble de clapets, sas, écluses, bols ou vases communicants » (p. I89). Le corps sans organes, c'est là où ça (se) passe, "on est déjà sur lui », mais, toujours se débordant, "on n'a jamais fini d'y accéder, c'est une limite» (p. I86). Jamais formé, il n'est jamais fermé: c'est un ensemble de virtualités et de «pratiques», un «voyageur du désert» (ibid.).

9. P.-A. Michaud, «Étienne-Jules Marey et la poétique des mobiles», Skteches, ouvr. cité, p. 55 .

IO. On m'excusera de laisser de côté pour la clarté de l'exposé les passages des uns aux autres.

II. G. Deleuze et F. Guattari, Mille plateanx, Paris, Minuit (Critique), I980, p. 633. 
Le cinéma doit pouvoir donner, dans la continuité du paradigme montagique aqueux rapidement esquissé plus haut, une expérience ruisselante des corps, au-delà de la pause picturale et photographique, iconicoindicielle, débordant la nature du montage figurant sur le corps figuré, une expérience du corps comme exsudation. Le corps au cinéma serait, à suivre la logique de Deleuze et Guattari, expressément, et uniquement, « rhizomatique» : corps non plus rapporté au régime chose / centre / point fixe, mais à des «directions mouvantes» (p.3I). Les corps de cinéma, même immobiles - ce qui n'est souvent, par ailleurs, qu'une appréhension relative due à nos seuils de perception -, parce qu'ils pourraient ne pas l'être (à la différence des corps de peinture ou de photographie), sont autant de lignes de fuite, me traversant, remontant le long de mes veines, se prolongeant dans les méandres de mes intestins, les alvéoles de mes poumons, les lobes de mon cerveau, les synapses de mes nerfs, le stroma de ma peau, «par variation, expansion, conquête, capture, piqûre» (p. 19). Comme la chaleur, dont parle Jacques Derrida dans La Carte postale, ces corps ont «envahi tout, à la fois très lentement et d'un seul coup, on ne sait plus, mais du dedans, jamais du lieu de la transfusion ». ${ }^{22}$

Le cinéma a agencé, distribué ses corps, dans chaque film, d'un film à l'autre, par connectivité, dérivation, effusion, par filature. Les corps cinématographiques ont surgi par meutes. Comme la ligne rhizomatique, qui caractérise les espaces lisses, le cinéma n'est pas une droite tirée apodictiquement d'un corps à l'autre (qu'ils soient des acteurs, des personnages, du réalisateur, de l'équipe technique, des spectateurs..., se combinant entre eux, tissant, tressant des écheveaux de laine, de toiles d'épeire, de lacets, de bretelles). Il ne peut que passer toujours entre les corps. Le cinéma manque tous les corps. Il en tracerait moins les contours (photographiques), ce qui ne serait encore que de la figuration optique (lignes du regard), qu'il ne les contourne, et les contournant, en délimite les pourtours entropiques.

\section{Le cinéma comme image du problème qu'est le mouvement: du mouvement à son engluement}

Néanmoins, les corps de cinéma, emportant avec eux les processus imageants, rabattant sur le montage, sont aussi susceptibles d'un mouvement qui ne se donne pas à appréhender sous le signe du rhizome, mais qui,

I2. J. Derrida, La Carte postale. De Socrate à Freud et au-delà, Paris, Flammarion, I980, p. I34. 
tout en étant moupement, relève d'un autre type de propagation, qui nous fera quitter la stricte orthodoxie deleuzienne, une propagation par calcification, sclérose, compaction, tassement, qui serait ce moment où le mouvement se lance tout en se présentant comme problème.

Qu'est-ce qu'un rhizome? Il suffira de rappeler ici qu'il permet de substituer au paradigme causal, rectiligne, mono-orienté et téléologique, caractéristique des espaces striés, des «plans d'organisation» et de la métaphysique traditionnelle (finissant toujours, peu ou prou, par remonter à une première cause unique), une explication labyrinthique, comme un lacis, une chevelure, un tallage ${ }^{\mathrm{I} 3}$ ne se laissant pas rapporter à l'unité ou à la multiplicité (ne sortant pas de l'idéal de l'unité), réseau fait d'embranchements incessants et de déviations successives qui maintient sauves les multiplicités. Au sein de la prolifération et de la dissémination rhizomatiques, tout est milieu et bord à la fois, chaque nodule, chaque joint, chaque glène n'est chaque fois qu'un «point quelconque », carrefour possible de directions nouvelles, toujours disponibles pour d'autres jugulaires tubéreuses, d'autres cheminements tubulaires. Visiter plutôt que viser. ${ }^{\text {I4 }}$ Dans le rhizome, il n'y a ni sujets ni substances, stables et fixes, il n'y a que du mouvement, autrement dit des vitesses, des quantités de mouvement (longitudes), et des intensités, des affects (latitudes).

Ma perspective se tiendra, pour le dire un peu brutalement, dans la déconstruction du mouvement rhizomatique. Cette déconstruction, au demeurant, n'est-elle pas déjà à l'œuvre dans le rhizome lui-même ? Aussi appellerai-je maintenant le moment liminaire d'arrêt qui lance tout mouvement, pour le distinguer de la dimension proprement rhizomatique du rhizome, tout en marquant ma dette à son égard, le rhizomique. Le rhizomique, qui est toujours inscrit dans le mouvement lui-même, est double: I / ce qui du mouvement préalablement se compresse, se rassemble pour qu'une propulsion, libératrice d'énergie, ait lieu et temps, comme dans le corps tout mouvement commence par un moment de crispation, de raccourcissement musculaire avant la détente, en vue de la détente, qui est déjà la détente et l'allongement (comme pro-jet du jet); 2 / ce qui, dans ce mouvement émergeant, vacille, hésite, adynamique, devant la précarité de l'étant et l'impermanence de ce qui ne se soutient que du jaillissement seul qui le constitue. Infinitésimal, différentiel, le rhizomique est ce

I3. Propriété de certaines plantes graminées qui produisent de multiples tiges, formant une touffe dense, à partir d'une plantule unique.

I4. J'emprunte cette opposition à J.-L. Nancy, À l'écoute, Paris, Galilée, 2002, p.59. 
qui des mouvements rhizomatiques d'emblée «se gèle» dans leur propre consistance pour que ces mouvements aient, à chaque instant, la puissance d'être les mouvements qu'ils sont - le rhizomatique consiste en son effusion -, dans le même temps que, venant à l'être, ils se jettent dans l'indétermination et l'extinction. C'est notamment cette dualité, toujours fendue d'un aspect à l'autre, qui inscrit le rhizomique comme stase immanente au mouvement.

\section{Empêtrements du mouvement}

Je donnerai, à partir de là, un exemple de ce caractère rhizomique des corps et des images de cinéma. Comme pour toute analyse, choisir un film pour illustrer mon propos ne fut pas chose facile, et ce pour deux raisons au moins - qui sont en fin de compte toujours les mêmes: d'une part, il fallait retenir un film suffisamment « ordinaire » pour qu'il n'ait rien d'une exception, trop restrictive pour faire expérience, et, d'autre part, comme n'importe quel film ne vaut pas, pour autant, n'importe quel autre pour situer le rhizomique, il me fallait distinguer un film dans lequel les processus imageants (visuels et sonores, seulement visuels pour ce qui m'intéressera ici) sont manifestement au service d'une intuition pertinente de ce qui était en jeu; donc un film somme toute peu ordinaire.

À cela venait s'ajouter le soin, comme le rappelle à fort juste titre Jacques Aumont dans $\grave{A}$ quoi pensent les films?, de ne pas plier les films, dans leur concrétude, aux réquisits d'un système a priori (et verbal) qu'on voudrait les voir vérifier. Quoique j'aie commencé par des remarques relativement théoriques, il s'agit là d'un ordre synthétique d'exposition, que l'on ne confondra pas avec l'ordre empirique des observations et des enquêtes. Il ne faut pas y voir un raisonnement déductif - tel qu'il se présente, uniquement pour la conviction de l'écriture et la clarté du commentaire -, mais bien une recherche inductive, lente et hésitante, principalement orientée par le souci de déterminer les problèmes que les films posent par leurs moyens propres, et non ceux que l'on ferait peser sur eux depuis un extérieur conceptuel.

Mon choix s'est finalement arrêté sur Le Lac des femmes de Yoshida Kijū (Onna no mizunmi, 1966). Me retiendra exclusivement une certaine scène à l'ouverture du film. En voici très succinctement le contexte: Miyako (Okada Mariko) est une jeune épouse infidèle qui vient d'accepter de poser nue pour son amant. Le soir même, rentrant chez elle, elle se fait dérober 
son sac à main, contenant les précieux négatifs - le moment est filmé avec la brusquerie d'un viol -, par un homme qu'elle prend d'abord pour un voleur à la tire.

Posons cela comme un modeste programme : le rhizomique, ou élan serrant / perplexité hasardée des mouvements, est donné à voir dans cette scène par différents mécanismes filmiques - au tournage et au montage -, lesquels vont constituer autant d'étapes progressives dans la mise en place d'une invention figurative rhizomique originale - qui n'en épuise nullement, cela va de soi, la diversité d'un film à l'autre. On pourra distinguer trois mécanismes en particulier, trois effets d'images qui vont mener à une approche visuelle du corps rhizomique dans cette scène du Lac des femmes: les effets de surplace, de pellicule et de vis.

\section{Le surplace}

La scène est constituée, pour l'essentiel, d'une même combinaison - une vue par-derrière et une vue par-devant, par rapport au corps de la femme -, qui se répète selon une cadence régulière : un plan par angle, à deux exceptions près - les deux plans d'introduction et une arythmie disruptive de gros plans à deux reprises - sur lesquelles la suite de ce texte reviendra.

Dans la première classe de plans (de moyens à rapprochés taille), on voit Miyako s'enfoncer de dos dans le noir total d'une rue (aucun détail d'objet ou de bâtiment n'est visible), seule éclairée - toute vêtue de blanc par ce que l'on supposera in fine, compte tenu du développement ultérieur de l'action, être une lampe de poche braquée, hors champ, par son poursuivant (la caméra est portée: nuance de bougé de reportage, style emprunté à Pasolini ou à Robert Aldrich, la femme adultère est assimilée par les conventions à une prostituée). L'impression est forte: celle d'un mouvement à la surface, inscrit dans nulle perspective illusionniste, dédiégétisé, dé-fictionnalisé, comme pour un personnage de flip book ou dans l'animation d'une série chronophotographique d'Étienne-Jules Marey. La femme ne semble pas avancer, mais bouger sans se déplacer - la caméra la suit, après l'avoir rejointe, en gardant la même distance -, mais pédaler dans un trou noir, "suspendue » dans un vide spatial angoissant - passant de la gauche à la droite de l'écran comme une figurine, découpée dans du papier, sur l'affiche où elle doit trouver place et être collée. Le mouvement, que Christian Metz tenait pour hautement substantialisant du fait qu'il détache une figure mobile d'un fond statique, tourne ici à vide en raison de l'élision du fond, seulement maintenu par la bande-son au 
titre de présence creuse (l'écho des talons de Miyako sur le bitume). On pense également devant ces plans quelque peu à une sorte de bunraku, le théâtre japonais de marionnettes plates et articulées, mais inversé: on ne voit plus l'ombre du personnage par transparence sur un écran translucide rétro-éclairérs, mais "l'ombre blanche», fantomale - une allusion à l'association traditionnelle femme/spectre? -, d'un corps sur un fond noir uniforme, la lumière du projecteur venant frapper l'écran de cinéma frontalement.

Ces plans sont montés en alternance avec des plans enregistrés face à Miyako, de l'autre côté de son corps pris comme point de symétrie sur le segment de sa trajectoire. On aura reconnu un «faux » raccord - en quoi est-il faux, au sens du faux pas? -, plus fréquent qu'on ne le croit, avec rupture de ce que l'on appelle l'axis of action; la règle classique voulant au contraire que, pour des raisons de croyance spectatorielle dans l'homogénéité de l'espace fictionnel, la caméra reste toujours du même côté de l'axe fictif qui oriente l'action, c'est-à-dire ni ne le franchisse ni ne stationne dessus. Cette rupture est accentuée par le contre-jour, le seul éclairage provenant, encore une fois, de «quelque part» derrière le dos de la femme - toujours hors champ, mais dorénavant parce que celé par son corps à l'avant-plan, réintroduisant si l'on y tient (imaginairement) de la perspective. De tels plans, quoique différents des premiers, produisent le même effet. On y croirait moins voir une femme qui marche, figure noire sur fond noir, que flotter des fibres blanches - le liseré de lumière sur toute la bordure de sa silhouette -, comme un filament d'ampoule, comme un grattage à la lame à même plusieurs photogrammes de la pellicule. Ainsi la femme, dont on devine quelques traits dans le noir, paraitelle de nouveau immobile, simplement déplacée parce que portée par un cercle incandescent comme une méduse par son ombrelle, corps désormais tiré par les cheveux - on en devine, irradiantes, quelques mèches.

Rhizomique 1 - Bouger sans se déplacer, être déplacé plutôt que se déplacer: tout mouvement est mouvement d'immobilisation.

I5. Dispositif qu'investira, avec une incroyable densité, Femmes en miroir (Kagami no onnatchi, 2002), superposant bunraku, traumatisme familial (impliquant mère, fille et petite fille), projection cinématographique et irradiations nucléaires de Hiroshima et Nagasaki (éblouissement, ombres projetées). 


\section{La pellicule}

Dans quelle matière exactement s'injecte le corps de Miyako, creuse-t-il, ronge-t-il, comme un ver xylophage et lucifuge (le Celluloïd n'est-il pas composé pour partie de camphre arboricole)? Car cette image n'est pas ici qu'une surface, elle a une épaisseur : le dernier plan, apportant désormais quelques données visuelles, mais déconnectées les unes des autres, de profondeur mimétique - la texture granulée d'une route «sur» la ligne de fuite, la masse plus claire d'un poteau téléphonique un peu plus loin sur la droite du cadre -, le confirmera avec l'arrêt de la caméra qui, ne suivant plus Miyako, la laisse s'éloigner, rapetisser dans le «fond» de l'image, s'y engloutir. De quoi le noir, ici, est-il l'image? Pour quoi vaut-il image?

Miyako n'est pas en effet n'importe quelle femme. Ici, il faut revenir un instant au récit. Miyako transporte sur elle un objet bien précis: des négatifs photographiques. Ceux-ci ne nous sont pas complètement inconnus: non seulement parce que nous avons précédemment observé Miyako les regarder dans la chambre d'hôtel, après que son amant l'a photographiée, mais surtout parce que nous les avons vus nous-mêmes, ou d'autres qui passent diégétiquement et rétrospectivement pour eux - même s'ils n'étaient pas présentés comme tels: au générique, en alternance avec les crédits, apparaissant justement dans le flash d'un fondu au blanc, comme sur le vif de la prise, avant de se dissoudre dans le noir sur fond duquel se détachaient les intertitres, également blancs. Cette précision permet de voir dans la question noire de la rue autre chose qu'une couleur informe, mais plutôt une sorte de métonymie extrêmement ingénieuse: le noir pour chambre de développement, le noir pour chambre de logement de la pellicule dans l'appareil (photo/caméra ${ }^{16}$ ), le noir, donc, pour camera obscura. La nuit comme appareil photo à ciel ouvert.

Trouverait-on cette association un peu hardie? Il faudrait alors revenir au deuxième plan de cette courte séquence. Celui-ci présente une trouvaille figurative, glissant d'une figuration mimétique vers une sorte de figuration récursive, qui va montrer que notre scène s'apparente bien à une mise en abyme pelliculaire. Qu'y voit-on? Un champ presque entièrement noir, ressemblant à une pièce quelconque pas éclairée?, à l'exception d'un double cadre, petit parallélogramme allumé sur la gauche faisant comme

I6. Le japonais, comme l'anglais, dit kamera dans les deux cas. 
une fenêtre mais paraissant plutôt être une cabine téléphonique bordée par le noir (qui n'est donc pas d'une pièce, mais d'une sorte de cache?...), à travers les vitres de laquelle on aperçoit Miyako remonter le trottoir de face, seule partie identificatoire du cadre. Ce plan très complexe et incertain ne fait pas que réduire l'écran - dans l'écran - et le déplacer, il est précisément fait pour rappeler, par un geste disruptif (déréalisant, déphotographiant) et figurativement fracturant, le support primitif de l'image. Est-ce trop forcer le trait que de voir, comme je tends à le proposer, dans la disposition de ce carré lumineux, là où il se trouve, avec le reste, une figure qui évoque un morceau de pellicule vierge accompagné de l'une de ses perforations, ce que l'on pourrait appeler pour l'occasion, bien que le vocabulaire en soit quelque peu flottant, un plan-photogramme, où, inversion encore, la partie perforée et la partie photosensible de la pellicule «échangent", figurativement parlant, leurs attributs, le site de la perforation portant désormais l'image? N'est-ce pas ce que Yoshida a recherché ici: l'invention, métaphorique, d'une image délogée de son espace chimique de figuration, expulsée là où ça enchaîne, où ça entraîne, où ça défile parce que l'image stricto sensu se défile, où ça permet de faire mouvement (la succession régulière et régulée des photogrammes au moment de la projection) : dans ce qui n'est après tout qu'un trou, lacune, non pas fausseté - illusionniste - mais fosseté organique.

Rhizomique 2- Tout mouvement est mouvement dans la pellicule et, plus précisément, là où rien ne s'image de la pellicule. Tout mouvement butte. Le cinéma image ses processus mécaniques dans l'élaboration de ses figures.

\section{La vis}

Par deux fois, la succession des plans à effet de surplace est détraquée par une série de gros plans sur le visage de Miyako se retournant vers son poursuivant. Précisons d'abord comment ces gros plans s'inscrivent dans la démarche pelliculaire rencontrée dans le point précédent. Le mouvement de la tête y est photographié selon la même alternance que celle précédemment examinée: d'abord plan de dos et rotation du visage, puis plan de face, en contre-jour, et rotation de la nuque. Mais la circularité produit désormais une symétrie spéculaire qui invite à voir, plus que les autres plans où Miyako ne latéralisait jamais son mouvement - de marche, 
donc toujours dans l'axe du viseur et de l'objectif de la caméra -, dans les gros plans de face/nuque le «négatif photographique» de ceux de dos/visage. Comme si cette jeune femme était moins vue par des yeux humains (cadreur, cinéaste, spectateur...) que par le film depuis son tréfonds, regard terrifiant qu'elle cherche à fuir : la lumière qui semble venir de l'homme qui la talonne n'est-elle pas aussi celle de l'éclairagiste qui la traque pour la faire rester dans le film? La caméra ne doit-elle pas toujours la doubler pour la prendre de plus loin et la garder dans le champ?

Mais ce n'est pas tout. Attardons-nous un peu sur la première occurrence de cette cellule, constituée de trois plans, en examinant tour à tour le passage du premier plan au deuxième, puis celui des deux premiers au troisième.

I. On remarque d'abord que le deuxième plan ne prend pas exactement la suite du premier. Le tour de tête y est raccordé en deçà de la position à laquelle l'avait suspendu la fin du plan précédent, à son point de départ, si bien que ce mouvement est intégralement répété durant le plan suivant - seule la position de la caméra a changé -, comme si tout en avait été effacé pendant le temps de la collure: mouvement bègue, impression de scratching, ou comme d'un saphir sur un microsillon rayé, mouvement qui déraille. Le «vis-à-vis» des plans (figurants) correspond à une vis du mouvement (figuré). L'effet sera le même lorsque la femme se retournera une seconde fois pour faire face à l'homme qui va bientôt lui arracher son sac à main.

2. Le troisième de ces plans semble revenir à l'angle de prise de vue du premier: nouveau mouvement de tête de la femme, qui dirige de nouveau son regard face à la rue et reprend sa marche. Toutefois, à y regarder de plus près, et comme le laissent deviner quelques traits perceptibles de son visage, nous n'avons pas changé de mouvement. Deux conséquences alors, très surprenantes, et qui ne sautent pas d'emblée à la vue: premièrement, lorsque Miyako avance, elle marche de dos en reculant vers la caméra, quand la suite la montrera cheminer le visage en avant, comme dans les plans précédents; deuxièmement, il s'agit d'une seconde répétition du même mouvement qu'au premier plan, qui ne se justifie pas, comme pour le deuxième, par le changement d'angle mais prend le premier à rebours comme par une simulation de rembobinage partiel de la pellicule - propagée par sa singulière mise en route, reculée -, de même que la présence diégétique de la pellicule photographique (dont le vol va lancer le film) avait déjà invité à revenir sur le générique, le positif développé précédant ici le négatif. Nous tenons là un plan, audacieux et névral- 
gique, réunissant en une seule, matricielle, toutes les figurations imageantes, toutes les fulgurations figuratives de la séquence.

Rhizomique 3-Se mouvoir: aller par soi, ne jamais aller de soi. Tout mouvement est l'affrontement de sa contrariété.

\section{L'image-déclenchement}

Que peut-on conclure de cette trop brève analyse? Rivé, gêné, sanglé, mis en pièces, incohérent, le mouvement y fait sens, non pas comme mouvement impossible - il se fait bel et bien -, mais comme difficulté de commencer à se mouvoir: lutte permanente contre l'inertie, bandage du corps toujours défait-refait ou fonctionnant à vide - pour se projeter, avancer malgré la paralysie devant l'ouverture des possibles pour arriver aux derniers pas, geste ou jet. Le mouvement n'est fait que de cela: de réfractions, de spasmes, de blocages évités de justesse, de déséquilibres chaque fois rattrapés, de fractures provisoirement réduites.

Quoi de plus remarquable alors, peut-être, dans cette scène de Yoshida que le corps de la femme y rejoue, et avec lui un certain traitement figuratif, les arrêts et relances perpétuels dont est fait l'entraînement de la pellicule dans la chambre de la caméra puis de la projectionneuse ${ }^{17}$ De quoi dure le film sinon de cet effort inconscient, et auquel on finit très vite, sinon d'emblée, par ne plus prêter attention, de la pellicule pour parvenir à défiler? Que faire, pour le rendre sensible, sinon déplacer notre perception anthropomorphique commune, déloger l'homme qui est dans notre regard vers une perception de cellulose, comme si la pellicule pouvait se percevoir elle-même, dans l'immanence de ce que l'on pourrait qualifier comme son imageance (effets de négatif, plan-photogramme), prolongeant le geste jusque dans l'arrêt sur tel plan du regard spectatoriel ? $^{18}$

I7. L'association de la femme avec le film est par ailleurs classique, se trouvant déjà, par exemple, dans Laura d'O. Preminger (1944) ou La Mort aux trousses (North by Northwest, I959) d'A. Hitchcock.

I8. Yoshida Kijū, «Composition verticale, étendue horizontale» (rédigé en I966), Logique de la négation de soi - Métamorphoses par l'imaginaire, Tōkyō, San.ichi Sōbō, I97O, p. 88: «Qu'est-ce qu'un film, en d'autres termes, capable d'immobiliser ce regard spectatoriel qui, horizontalement, glisse indéfiniment? Un film où le regard ne suscite pas simplement une critique facile, mais s'élève jusqu'au niveau d'une création?» Je remercie Mathieu Capel pour avoir porté ce texte à ma connaissance et pour la présente traduction. 
Telles sont les inventions d'images qu'on trouvera dans Le Lac des femmes, non pas pour donner à voir ce que j'ai appelé à cette occasion rhizomique - la pensée «des» images n'a rien d'intentionnel-, mais parce qu'elles rendent possible, par leur passibilité, par leur entrelacs, l'agencement idiomatique d'une perception d'images dans laquelle le mouvement se met en question. Ces quelques plans, sans prétendre faire valoir avec un film un fait générique: il faudrait en examiner plusieurs autres, tendraient à laisser voir qu'en cinéma, en dépit des apparences, c'est moins le mouvement stabilisé et assuré - un tel mouvement existe-t-il seulement, quel qu'il soit? - que le mouvement comme apparition-disparition toujours continuée, qui fait image, mouvement mouvementé pour lequel le commencement n'en finit jamais de commencer. Le cinéma, ou l'art des déclenchements inlassablement reconduits, l'art de chaque instant comme envoi du temps. 


\section{::: \\ La forme et l'informe: de la dissolution du corps à l'écran}

Le verbe s'efface devant le bruit, le motif disparait dans le grain. Nous devinons les formes par ce qui les entoure, non par ce qu'elles sont, nous comprenons la situation par ses conséquences, non par sa nature. Nous cherchons une forme familière à laquelle nous raccrocher mais elle se dérobe aussitôt. Chaque image est empreinte d'un manque qui déforme son environnement tout en l'aspirant, comme un trou noir. Elle nous attire aussi, nous invitant à fouiller, attisant nos projections, extrayant notre propre matière pour se combler.

A. Gimel, «La forme soustraite», La Vie nouvelle: nouvelle vision.

Dans l'une des premières séquences du film de Philippe Grandrieux La Vie nouvelle (2002), surgit une suite d'images éphémères mais saisissantes: une silhouette humaine apparait, très floue, qui semble courir vers la caméra, comme si elle tentait de s'arracher à l'arrière-plan indistinct qui menace de l'engloutir, de se détacher de la matière même du film. Le bourdonnement sourd de la bande sonore accompagne la trajectoire incertaine et les distorsions de cette forme aux contours changeants, qui, telle une tache mouvante sur la pellicule celluloïd, parait sur le point de se dissoudre dans la texture neigeuse qui emplit le reste du cadre. ${ }^{I}$

I. Le contenu de cette contribution émane en partie d'un projet plus large qui a fait l'objet d'une publication en 2007: M. Beugnet, Cinema and Sensation. French Cinema and the Art of Transgression, Édimbourg - New York, Edinburgh University Press - Columbia University Press, 2007. 
Grandrieux fait partie de ces quelques cinéastes français contemporains qui, en réinjectant à l'univers du long métrage une dose salutaire d'expérimentations visuelles et sonores, radicalisent à nouveau notre expérience de spectateur, la manière dont nous regardons, ressentons et pensons par le cinéma. Ses longs métrages font partie de ce type de cinéma hybride qui, naviguant entre film expérimental et cinéma classique («la zone bâtarde du cinéma français", comme l'appelle Dominique Noguez²), participe, à sa manière, à un retour à une corporéité filmique. ${ }^{3}$ C'est un cinéma de la sensation; un cinéma qui fait la part belle à la matérialité des corps et des objets mais aussi à la matière des images et des sons eux-mêmes. Ici, les techniques cinématographiques, mises au service des qualités sensorielles du film, participent à l'élaboration d'une synesthésie, plus précisément d'une vision «haptique » : il ne s'agit plus seulement de construire un espace correspondant au regard distancié de l'observateur omniscient, et destiné à une interprétation principalement narrative et fondée sur la représentation, mais aussi et surtout, en jouant sur les textures et les densités visuelles et sonores, de faire fonctionner notre « œil tactile».4

Flou, brouillage, ou au contraire saturation de détails et hyper-précision photographique, extrêmes gros plans, surimpressions, sous-expositions et surexpositions, variations d'intensité et de tonalités sonores: autant de techniques qui génèrent un monde d'images et de sons vacillant entre le figuratif et l'abstrait, et où la figure humaine, en tant qu'entité autonome, tend à perdre sa fonction de principal point de référence, voire à se dissoudre entièrement.

$\mathrm{Si}$ le processus est familier dans le cinéma expérimental et d'animation', il est généralement proscrit du long métrage, où la composition de l'image et du son reste communément gouvernée par les conventions du réalisme photographique et par une organisation spatio-temporelle visant au respect de la perspective et de la clarté du récit. De là découle sans doute le caractère subversif propre dont jouissent les procédés de décentrage et de dissolution de la figure humaine dans le cinéma de format classique. Comme nous allons le voir, certains films créent d'ailleurs

2. D. Noguez, Éloge du cinéma expérimental, Paris, Paris expérimental, I999, p. I85.

3. J. Game, «Cinematic bodies. The blind spot in contemporary French theory on corporeal cinema ", Studies in French Cinema, vol. I, n ${ }^{\circ}$ I, 200I, p. 47-53 et particulièrement p. 48.

4. P. Bonitzer, Le Champ avengle. Essais sur le réalisme au cinéma, Paris, Cahiers du Cinéma, I999, p. 28.

5. Voir M. Beugnet, "Experimental French cinema: the figural and the formless », Avantgarde Cinema, A. Graf et D. Sheuneman éd., Amsterdam, Rodopi, 2007. 
d'étranges moments d'inversion: c'est l'inerte, le paysage ou l'objet inanimé, qui semble détenteur du regard, et le sujet - la forme humaine à laquelle le spectateur s'identifie - qui, pris dans son champ de vision, semble se décomposer.

C'est à certaines manifestations et ramifications théoriques de ce processus de dissolution, de ce passage du figuratif à l'informe où la forme humaine s'atrophie, que je propose de consacrer les pages qui suivent, inspirées de séquences extraites des longs métrages de Philippe Grandrieux, Bruno Dumont, Léos Carax, Arnaud des Pallières, Pascale Ferran, Douglas Gordon et Philippe Parreno.

\section{De la figuration à l'abstraction et à l'informe}

Dans le cinéma expérimental abstrait, la forme humaine n'a pas sa place, elle s'est dissoute entièrement dans l'image-matière. Le cinéma figuratif (narratif) classique opère un autre processus d'abstraction, ou de conceptualisation : le corps du personnage-acteur est littéralement désincarné; il devient signe, au service de la lisibilité de l'image-texte. ${ }^{6}$ C'est entre ces deux pôles qu'Antonin Artaud, dans un texte écrit en 1927, situe la troisième voie du cinéma - celle qui correspondrait à la vocation authentique de l'art des images en mouvement:

Deux voies semblent s'offrir actuellement au cinéma, dont aucune certainement ne peut revendiquer être la meilleure. Le cinéma pur ou absolu, d'une part, et de l'autre, cette sorte d'art véniel hybride qui s'obstine à traduire en images plus ou moins heureuses des situations psychologiques qui seraient parfaitement à leur place sur une scène de théâtre ou dans les pages d'un livre, mais pas sur l'écran, n'existant guère que comme le reflet d'un monde qui puise ailleurs sa matière et son sens. Entre l'abstraction visuelle purement linéaire (un jeu d'ombres et de lueurs est comme un jeu de lignes) et le film à fondements psychologiques qui relate le

6. V. Amiel résume ainsi le processus de dématérialisation des corps dans le cinéma classique: «Dans sa dimension classique, c'est-à-dire narrative et romanesque, le cinéma instrumentalise le corps, le défaisant de cela même qui le tient, pour n'en donner plus que l'idée. [...] Obligé de privilégier l'effacement, il joue d'une figuration qui se suffit à ellemême; il dématérialise les corps pour n'en faire plus que des éléments de reconnaissance, de l'ordre de l'idée» (V. Amiel, Le Corps an cinéma. Keaton, Bresson, Cassavetes, Paris, PUF, I998, p.3). On pense aussi aux commentaires de T. Docherty qui, se référant à J.-F. Lyotard, parle de «la priorité donnée au "lisible" sur ce qui est simplement "visible", de sorte qu'on nie la visibilité du visible, la traduisant sans cesse en ce qui, par essence, peut être appréhendé comme "texte" ( (T. Docherty, Alterities. Criticism, History, Representation, Oxford, Oxford University Press, I996, p. I55, traduction M. Beugnet). 
développement d'une histoire dramatique ou non, il y a une place pour un effort vers le cinéma véritable dont rien dans les films jusqu'ici présentés ne fait prévoir la matière ou le sens.7

À l'autre bout du Xx' siècle, Vincent Amiel lui fait écho lorsqu'il s'interroge sur la manière dont s'effectue, au cinéma, le passage de la matière au symbolique, du matériau audiovisuel à la représentation:

Car si l'image est une présence, d'abord lumière et matière, si ces couleurs sont à nos yeux de véritables objets, comme les formes et les mouvements qui l'animent, comment les transformer assez en représentation sans qu'ils ne deviennent pour autant, à l'instar de la plupart des mots, de simples «renvois $»$ ?

Dans Logique de la sensation, le philosophe Gilles Deleuze s'était également lancé dans une réflexion sur la figuration et l'abstraction, en s'attachant lui aussi à décrire une autre voie - celle par laquelle l'art moderne s'engage au-delà de la figuration sans pour autant s'identifier à l'abstraction pure.

Il a fallu l'extraordinaire travail de la peinture abstraite pour arracher l'art moderne à la figuration. Mais n'y a-t-il pas une autre voie, plus directe et plus sensible??

L'analyse que développe ici Deleuze est une introduction à son traité sur la peinture de Francis Bacon. Elle semble pourtant tout aussi appropriée dans le contexte de l'art cinématographique, puisque le cinéma est un art du temps et de la fluctuation où l'image, comme le son, est en constante mutation. L'ensemble des techniques élaborées par le cinéma classique $^{\mathrm{IO}}$ vise d'abord à positionner le regard et son objet, et à simuler une relation de sujet à objet qui serait stable, voire immuable. Pourtant, parce qu'ils sont saisis dans la durée, les éléments de figuration, au cinéma, ne sont jamais fixés; ils se font et se défont au rythme des images et au gré de leurs modifications internes - lumière, grain, point de vue, angle de prise de vue... Ainsi, par-delà la référence picturale, la description que donne Deleuze d'un art qui échapperait aux règles figuratives,

7. A. Artaud, «La coquille et le clergyman» [1927], Euvres complètes, vol. III, Paris, Gallimard, I978, p.2I.

8. V. Amiel, Le Corps au cinéma, ouvr. cité, p. 4.

9. G. Deleuze, Francis Bacon. Logique de la sensation, Paris, Seuil, I98I, p. 19. Voir aussi J. Aumont, Matière d'images, Paris, Images modernes, 2005.

IO. Ce qu'on appelle pertinemment en anglais le continuity system, c'est-à-dire les procédés permettant de maintenir la continuité spatiale et temporelle qui caractérise les dispositifs de narration filmique classique. 
illustratives et narratives crée d'indubitables résonances dans le contexte d'un «cinéma de la sensation $»^{\mathrm{I}}$ :

Il y a deux manières de dépasser la figuration (c'est-à-dire à la fois l'illustratif et le narratif): ou bien vers la forme abstraite, ou bien vers la Figure. Cette voie de la Figure, Cézanne lui donne un nom simple: la sensation. La Figure, c'est la forme sensible rapportée à la sensation [...] à la fois je deviens dans la sensation et quelque chose arrive par la sensation, l'un par l'autre, l'un dans l'autre. Et la limite, c'est le corps qui la donne et qui la reçoit, qui est à la fois objet et sujet. [...] C'est pourquoi la sensation est maitresse de déformations, agent de déformations du corps. (Ibid., p. 39-40)

Dans l'étude de Deleuze, le domaine de la figure, par contraste avec celui de la figuration, correspond à un espace où la forme, humaine, est présente et, initialement au moins, reconnaissable, mais apparaît indépendamment des structures spatio-temporelles liées à la fois à la perspective et au narratif. La figure humaine demeure donc un élément clé de la composition, mais elle est isolée de tout contexte narratif direct et présentée dans un espace où la perspective est absente ou mise à mal - où la relation entre figure et fond ne se définit pas par la profondeur, mais en tant que coexistence sur un même plan. Figure et fond sont ainsi «saisis par et dans une vue proche, tactile ou "haptique" ", dans une "corrélation de deux secteurs sur un même plan également proche» (p. I4).

Ces commentaires, par lesquels Deleuze anticipe le passage du formalisme moderniste à «l'informe » postmoderne tel qu'on peut le repérer dans les œuvres des artistes et les écrits des théoriciens de l'art contemporains $^{12}$ sont pertinents non seulement dans le domaine du cinéma expérimental mais aussi, comme nous allons le voir ici, dans le contexte d'un cinéma de la corporéité et de la sensation oscillant entre cinéma expérimental et cinéma classique. En effet, ce qui est en jeu ici, c'est à la fois le passage, ou plutôt la fluctuation entre figuration et figure rendue possible par l'élaboration d'une "vision haptique », et ses implications. Car mettre en valeur la matérialité de l'image et du son au cinéma, c'est brouiller la frontière entre sujet et objet, figure et fond - c'est-à-dire la base même de

I I. L'expression "cinéma de la sensation» n'est nullement destinée à donner l'impression qu'il s'agit d'un mouvement, d'un genre, ou même d'une catégorie établie de films. Elle signale simplement une tendance, approfondie ou ponctuelle selon les films, à privilégier l'aspect matériel du film.

I2. H. Foster, The Return of the Real, Cambridge (MA), MIT Press, I996; R. Krauss, The Originality of the Avant-Garde and Other Modernist Myths, Cambridge (MA), MIT Press, I985; R. Krauss et Y. Bois, Formless. A User's Guide, Cambridge, Zone Books, 1997. 
notre conception et de notre représentation du moi comme entité indépendante. La vision haptique et le déni de la perspective perturbent la hiérarchie visuelle conventionnelle qui érige la figure humaine en entité autonome et la place au centre de la représentation.

\section{L'haptique et l'optique}

Plus que jamais depuis la naissance du cinéma, la critique du mode de perception et de représentation dominant - le mode «optique», fondé sur la perspective, et que le cinéma classique adopte comme modèle - est au centre des discussions sur les systèmes épistémologiques occidentaux. Si l'argument est familier au point d'en devenir un truisme de la pensée contemporaine, il n'en garde pas moins sa pertinence dans le contexte des systèmes de représentations d'aujourd'hui: le régime de représentation optique défini par les règles de la perspective et de la distinction entre figure et fond implique le principe d'un sujet cartésien, autonome et rationnel, qui pose sur le monde un regard objectif distancié. Certains théoriciens de l'art, parmi lesquels Deleuze, ont eu à cour de rappeler que ce modèle épistémologique et représentationnel, quoique dominant, n'en est pas moins historique:

Si l'on peut parler d'une représentation classique, c'est au sens de la conquête d'un espace optique, à vision éloignée qui n'est jamais frontale: la forme et le fond ne sont plus sur le même plan, les plans se distinguent, et une perspective les traverse en profondeur, unissant l'arrière-plan au premier plan; $[\ldots]$ le contour cesse d'être limite commune sur le même plan pour devenir autolimitation de la forme ou primauté de l'avant-plan. [...] L'art peut alors être figuratif, on voit bien qu'il ne l'est pas d'abord, et que la figuration n'est qu'un résultat. ${ }^{\mathrm{I3}}$

Comme Deleuze, Laura Marks fait référence aux travaux des historiens de l'art du XIX ${ }^{\mathrm{c}}$ siècle (en particulier aux écrits d'Aloïs Riegl, dont Deleuze extrait notamment le terme «haptique $»^{14}$ ) et contraste l'espace haptique avec celui qui s'est élaboré en privilégiant une construction spatiale conceptuelle, fondée sur la distance et la profondeur:

I3. G. Deleuze, Francis Bacon, ouvr. cité, p. II8.

I4. "Riegl et Auerbach [... ont décrit comment l'art occidental est parvenu à instaurer une relation de similarité sensorielle avec son objet par la représentation plutôt que par le contact» (L. Marks, The Skin of the Film. Intercultural Cinema, Embodiment and the Senses, Durham, Duke University Press, 2000, p. 167). 
De façon déterminante, c'est la création d'un espace abstrait dans l'art byzantin qui permet au détenteur du regard d'identifier les figures non plus comme des éléments concrets sur une surface, mais comme des figures dans l'espace. L'espace haptique, au contraire, est concret : c'est sur la surface, et sur elle seule, qu'il cherche à établir une unité. L'apparition de la représentation optique marque un mouvement général vers un idéal culturel d'abstraction qui aura des conséquences importantes. Le principe d'abstraction a favorisé la création d'un plan pictural basé sur l'illusion et permettant l'identification de, et avec, les figures dans le sens où nous comprenons «identification» de nos jours. Autrement dit, la représentation optique a permis d'établir une plus grande distance entre sujet et objet, grâce à laquelle le sujet peut désormais se projeter, en imagination, dans et sur l'objet. ${ }^{\mathrm{Is}}$

Tandis que le régime de représentation optique crée un espace en profondeur duquel se détachent des éléments de figuration autonomes, le mode haptique ignore la hiérarchie qu'instaure l'effet de perspective entre les objets de la perception. Dans le mode haptique, l'attention va aux détails tactiles et à la surface matérielle de l'image où figure et fond fusionnent. Les images haptiques encouragent ainsi un mode de perception visuelle proche du sens du toucher, où l'œil, rendu sensible à l'aspect concret de l'image, enregistre des qualités habituellement associées au contact avec la peau: "Enfin on parlera d'baptique [...] quand la vue elle-même découvrira en soi une fonction de toucher qui lui est propre, et n'appartient qu'à elle, distincte de sa fonction optique. ' $^{16}$ Le son n'a évidemment pas sa place dans l'étude de Deleuze; il n'en est pas moins aisé de rattacher la notion d'haptique à un espace sonore déhiérarchisé, où les voix et les dialogues, par exemple, ne seraient qu'un élément parmi d'autres de la bande-son.

Marks fait partie des théoriciens qui, dans la filiation de Deleuze, rejettent le principe communément accepté selon lequel la représentation optique est l'aboutissement d'un processus logique qui mène à l'établissement des règles de la perspective comme seul système de représentation figurative valable. Mais, de manière cruciale, c'est dans le cinéma que Marks trouve la confirmation de la persistance et de la pertinence de la vision haptique ${ }^{\mathrm{I7}}$ :

I 5. Ibid., p. I66, traduction M. Beugnet.

I6. G. Deleuze, Francis Bacon, ouvr. cité, p. I46.

I7. Voir aussi, entre autres, P. Bonitzer, Le Champ avengle, ouvr. cité, et V. Sobchack, Carnal Thoughts. Embodiment and Moving Image Culture, Berkeley - Los Angeles, University of California Press, 2004. 
[...] nous retrouvons ce qui intéressait les théoriciens du cinéma moderne, comme Benjamin, Béla Balázs, et Dziga Vertov : la relation intime entre le corps du spectateur et l'image cinématographique, reliant ainsi des époques de la théorie du cinéma que séparent des décades de théorie dominée par les modèles linguistiques. (p.I7I)

C'est à la fois dans la théorie et dans la pratique, comme le souligne Jérôme Game, et au cour même du domaine du cinéma classique - le long métrage de fiction - que l'on trouve des exemples de ce fertile retour vers un cinéma envisagé dans sa corporéité, en tant que matière et sensation, comme espace/surface haptique. ${ }^{18}$ Les films mentionnés dans cet article font ainsi partie d'un large corpus d'œuvres où, de manière systématique ou ponctuelle, une esthétique de la sensation défait l'organisation optique classique.

Dans le cinéma classique, regarder un film, c'est distinguer des formes humaines (des personnages) et des objets tels qu'ils apparaissent dans l'espace de la représentation, éléments à la fois distincts et déjà codifiés du récit filmique que le spectateur appréhende de son point de vue d'observateur omniscient. Un cinéma de la sensation joue au contraire sur les qualités matérielles du médium pour construire un espace déhiérarchisé où peuvent se former des relations d'intimité et de proximité entre spectateur et objet du regard. Dans cet espace, l'identification primaire, avec le film même en tant qu'objet sensoriel, peut prendre le pas sur l'identification secondaire, avec les personnages. Changement de focale et de mise au point, juxtaposition de différents types de supports (film de 8 , I6 ou 35 millimètres, numérique, haute et basse définition...) permettant des variations de grain et de densité ; angles inhabituels de prise de vue et de cadrage; montage rythmique ou plastique, etc. : le choix de certaines techniques permet d'opérer un passage de l'optique à l'haptique, où la présence matérielle de l'image entre en concurrence et parfois prend le pas sur ses qualités représentationnelles. Comme le remarque Marks, les images haptiques, «lorsqu'elles deviennent figuratives, ne le deviennent que graduellement» (p. I63). Amoindrir la lisibilité d'une séquence, c'est offrir au spectateur la possibilité d'une expérience plus sensuelle et moins contrôlée avec l'objet-film. Mais c'est aussi le priver de ses repères, et en particulier du point d'ancrage que représente le corps du personnage comme objet de projection et comme relais interne du regard du spectateur - expérience d'autant plus perturbante lorsqu'une relation d'iden-

I8. J. Game, «Cinematic bodies», art. cité, p. 46. 
tification classique s'est établie auparavant. Il n'est pas surprenant que le cinéma d'horreur, en particulier, use et abuse - dans la mesure où ceuxci deviennent de simples conventions de genre - des effets de la visualité haptique, puisqu'elle souligne à la fois la vulnérabilité du corps et la facilité avec laquelle la forme humaine, entité distincte, enveloppe du moi, peut être rendue à la matière indistincte.

À la fois trop proche de la notion de concept, et trop loin de la sensation, le terme "abstraction» semble décidément inadéquat à décrire ce passage de la figuration à l'indifférenciation : abstraire, c'est mettre à part, isoler. C'est donc la vision et l'expression de Georges Bataille qui s'impose ici ; terme plus obscur qu' «abstraction », et qui a aussi l'avantage de juxtaposer en un même mot la forme et sa négation, l'«informe» semble plus apte à rendre l'ambiguïtér' qui est au cœur du processus décrit ici : l'évocation d'une attraction du néant où la notion même de sujet se désagrège, l'oscillation entre les plaisirs de la communion sensorielle et la terreur de l'être en soi qui se dissout. ${ }^{20}$ Hal Foster décrit l'informe selon Bataille comme «la condition où la forme signifiante se dissout parce que la distinction fondamentale entre figure et fond, je et l'autre, a disparu ». ${ }^{2 \mathrm{~T}}$ Pour Foster, l'informe est indissociable de certaines des intuitions lacaniennes. Envisagé comme l'évocation d'une individualité qui se désagrège sous le regard inerte du «Réel $»^{22}$, il renvoie à la fois au «Réel troumatique» de

I9. On pourrait aussi évoquer la notion d'«abject» telle que la décrit J. Kristeva, Pouvoirs de l'horreur. Essai sur l'abjection, Paris, Seuil, I980.

20. Au début du $\mathrm{xx}^{\mathrm{e}}$ siècle, W. Worringer décrit la tendance à l'abstraction (dans le sens d'«extraire») qui se manifeste à certaines périodes de l'histoire de l'art comme l'expression d'une profonde anxiété existentielle qui résulte dans un désir d' «abstraire» les objets de la représentation au chaos qui les entoure. Au contraire, les mouvements artistiques qui tendent à intégrer harmonieusement les objets dans leur environnement s'offrent au spectateur comme une expérience de plénitude existentielle (einfühlung). Dans la même veine, les modèles psychanalytiques, ainsi que les approches inspirées par les écrits de G. Bataille, s'attacheront surtout à trouver, dans le passage de la figuration à l'indifférenciation ou à l'«informe ", l'expression de l'angoisse d'un anéantissement possible du moi. Au contraire, la phénoménologie de M. Merleau-Ponty et les écrits de G. Deleuze, notamment en collaboration avec F. Guattari, forment la base d'une réflexion plus positive, où la diminution de subjectivité signifie aussi la possibilité d'une relation plus riche au monde. Entre ces deux pôles s'ouvre un champ particulièrement fertile d'intuitions dont se nourrit aussi l'univers du cinéma de la sensation. W. Worringer, Abstraktion und Einfühlung: ein Beitrag zur Stilpyschologie, Munich, R. Piper, 1908.

2I. H. Foster, The Return of the Real, ouvr. cité, p. I49, traduction M. Beugnet.

22. Dans la terminologie lacanienne, le «Réel» est proche de l'«informe» de Bataille: le Réel est ce qui échappe à la fois à l'imaginaire et au symbolique, ce qui ne saurait être entièrement symbolisé et réduit en discours ou représentation. Le Réel correspond au règne de l'homogène ou de l'indifférencié. 
Lacan, et à sa description du sujet dépossédé de sa condition de sujet, et devenant simple «tache» dans le «spectacle du monde». ${ }^{23}$

\section{La tache, le trou, le corps dissous}

Étrange road movie où le récit, soumis au rythme de la répétition et des longs plans-séquences, «semble faire du sur-place »²4, le Twentynine Palms de Bruno Dumont (2003) décrit la trajectoire d'un couple dont la relation osmotique est mise à l'épreuve de la solitude et du vide. Personnage principal du film, le désert, filmé en scope, est comme une présence implacable, à la fois visuelle et sonore, témoin et catalyseur de la désagrégation de la relation humaine. La bande-son joue ici, remarque Philippe Rouyer, un rôle crucial: «Les bruits englobent les personnages. Dumont n'hésite pas à rendre, par exemple, une discussion inaudible du fait de son environnement sonore» (ibid., p.I6). Le paysage enveloppe ainsi le couple dans un rapport de plus en plus ambigu entre fond et forme, où la figure humaine parait alternativement réduite, absorbée, voire invalidée. L'une des séquences tournées sur la route crée ainsi un curieux hiatus visuel. Le couple, filmé de dos dans une voiture, a un échange d'une inquiétante banalité: «À quoi tu penses? - À rien. » Silhouettées sur un fond de paysage blanchi par la lumière, la tête du conducteur et celle de sa passagère se dessinent dans le cadre telles deux taches sombres masquant la vue. On retrouve ce troublant motif visuel dans le long métrage suivant de Dumont, Flandres (2006). À la fin de la première scène d'amour entre les deux personnages principaux, la caméra s'attarde sur les amants allongés l'un sur l'autre, écrasés contre le sol par l'objectif qui les cadre en plongée. Tandis que la jeune femme affronte sans ciller le regard de la caméra, la tête de son amant, recouverte d'un bonnet noir, forme un trou sombre au centre de l'image.

Plus encore que la mise en scène classique de l'annihilation physique telle que nous la proposent les films de genre, la dématérialisation de la forme humaine - qui devient tache ou trou et semble voiler ou crever la surface de l'image - est l'expression visuelle d'une subjectivité qui oscille entre présence et néant. Le contrechamp appartient alors à l'inanimé, au regard inerte qui nie la forme humaine prise dans son champ de vision.

23. J. Lacan, Le Séminaire, livre XI, Les Quatre Concepts fondamentaux de la psychanalyse, Paris, Seuil, 1973, p. 73-74.

24. P. Rouyer, "Twentynine Palms: wild at heart», Positif, n' ${ }^{\circ}$ II, 2003, p. I5-I6 et particulièrement p. 15 . 
Dans les films de Dumont, ce rôle échoit aux paysages et aux machines. Ainsi, au début de Twentynine Palms le face-à-face annonciateur du couple et des éoliennes, où les personnages, fascinés par l'inexorable rotation des pales géantes, semblent saisis à la fois d'admiration et d'effroi devant la puissance des machines. La terreur nait, souligne Kent Jones, de ces moments où l'objet semble retourner le regard des personnages ou de la caméra, sans offrir de réponse à leur questionnement implicite :

Dumont utilise le champ/contrechamp classique, mais il reste sur l'objet pendant une durée inconfortable sans que l'image révèle quoi que ce soit pour autant: au contraire des quatre-vingt dix-neuf pour cent des films que nous voyons, il n'y a, chez Dumont, aucune connexion psychologique ou poétique entre celui qui regarde et l'objet de son regard. De telles séquences d'images sont imbues d'une énergie terrible : un désir élémentaire, basique, que le monde fasse sens. ${ }^{25}$

C'est grâce à son aptitude à accorder une place égale et interchangeable aux objets et aux êtres animés, au subjectif et à l'objectif, que le cinéma peut rendre manifeste ce que l'entendement peine à saisir : l'intuition d'un regard qui échappe au sujet et menace son hégémonie. Deleuze et Guattari, dans la filiation de Béla Balász, reconnaissent au cinéma une capacité inégalée à rendre au monde des objets son regard, qui se manifeste notamment dans un processus de visagéification ${ }^{26}$ :

Même un objet d'usage sera visagéifié: d'une maison, d'un ustensile ou d'un objet, d'un vêtement, etc., on dira qu'ils me regardent [...]. Le gros plan de cinéma porte aussi bien sur un couteau, une tasse, une horloge, une bouilloire, que sur un visage ou un élément de visage; ainsi Griffith, la bouilloire me regarde. ${ }^{27}$

Le gros plan n'est pas le seul moteur ici : le processus s'applique aussi bien à un objet qu'à une maison. Comme le démontrent les univers cinématographiques de Dumont, de Grandrieux ou de des Pallières, pour ne nommer que les réalisateurs dont les films sont cités ici, même avant la dissolution de la figure humaine, un paysage naturel ou urbain peut

25. K. Jones, «L'Humanité», Film Comment, vol. 36, nº 6, 2000, p. 73, traduction M. Beugnet.

26. Pour Deleuze et Guattari, l'effet de visagéification opère véritablement lorsqu'un détail saisi en gros plan, ou l'image d'un objet inanimé, se trouve pris dans un processus de déterritorialisation et d'abstraction où se retrouve la notion du «trou noir». La transformation (en visage ou en paysage: un objet ou un visage peut être paysagéifié) met en jeu l'aspect physique de l'image telle qu'elle est perçue - ce que Deleuze et Guattari appellent le processus «mur blanc - trou noir» (G. Deleuze et F. Guattari, Mille plateaux. Capitalisme et schizophrénie 2, Paris, Minuit, I980, p.2I4-215).

27. Ibid., p.2I4. 
s'affirmer à l'image non comme simple fond, mais comme une présence indépendante du réfèrent humain et de la notion d'échelle qui prend généralement le corps de l'homme comme unité de base. Absence de la figure humaine, attention dévolue à l'objet et à la matière, effet du scope où la silhouette peine à s'imposer, du zoom qui écrase la figure contre le fond, angles de prise de vue et décadrages, contraste entre haute définition photographique et flou - autant de techniques qui contribuent à déstabiliser le regard et s'attaquent à l'intégrité de la forme humaine.

L'une des séquences les plus terrifiantes de La Vie nouvelle ne contient d'autre violence que celle, implicite, de la vision qu'elle offre de la place de l'humain dans le monde contemporain. Le contexte est celui des Balkans, une zone hantée par le souvenir et les fantômes d'un conflit meurtrier où, à la périphérie d'une grande ville sans nom, dans un hôtel qui sert de bordel, un soldat américain - peut-être de retour du Kosovo - parachève sa descente aux Enfers. La séquence en question est filmée dans un couloir de l'hôtel, lieu anonyme, sans repère - un «espace quelconque». La première partie s'annonce un peu comme une variation sur un thème de cinéma expérimental: un plan très long, qui cadre le corridor en légère contre-plongée, et progresse lentement vers l'unique fenêtre qui perce le mur au fond du couloir, au centre du cadre. L'inéluctabilité du mouvement, sa constance mécanique créent un puissant effet d'emprisonnement. Un paysage urbain désolé apparaît, se découpant dans l'encadrement sombre de la fenêtre avec la précision et l'immobilité d'une photographie en haute résolution. Le paysage emplit bientôt tout le cadre: perspective barrée par des strates de béton, immeubles sans âme perforés de rangées de fenêtres identiques dont le regard inerte retourne celui de la caméra. Le plan s'interrompt, et la séquence reprend avec une vue du même corridor, mais qui inclut cette fois la silhouette du soldat américain debout devant la fenêtre: forme sombre contre la lumière blême, elle semble comme dissoute par la force concentrée et indistincte du regard de la cité.

\section{Liminalités}

Ce jeu sur les rapports entre la forme et le fond, où le corps humain saisi dans le champ figural semble en porte-à-faux avec ce qui l'entoure, est un élément crucial de l'univers cinématographique de Grandrieux. La figure humaine y apparait comme piégée entre deux niveaux d'apparence, et sans cesse soumise à la force d'attraction de l'informe. Le passage entre l'inexo- 
rable concrétude de la photographie haute définition à la confusion du flou et de la surimpression évoque une réalité en état de flux. La figure et le fond, l'interne et l'externe, le corps individuel, subjectif, et son environnement objectif, y paraissent tantôt désynchronisés, tantôt proches de la fusion. Grandrieux décrit la composition du son de ses films, comme celle des images, en termes de modelé: «Le son s'est fabriqué par couches, épaisseurs successives, comme une pâte sonore qui enveloppe le film. $\gg^{28}$ Les dialogues, dans Sombre (1999), et plus encore dans La Vie nouvelle, ne sont pas un élément prédominant. Comme dans les images, la trace sonore de la présence humaine fait souvent corps avec la matière, la rumeur et les grondements qui se mêlent à la musique et aux compositions électroniques et circulent à travers la bande-son comme une respiration.

Ni Sombre, singulier portrait d'un tueur en série, ni La Vie nouvelle ne sont structurés comme une progression classique, un mouvement linéaire qui mènerait d'un niveau de réalité familier aux profondeurs obscures cachées sous la surface de cette réalité. C'est la matière audiovisuelle ellemême qui est en flux constant; aussi les distorsions auxquelles sont soumises les images, qu'elles soient dues aux variations de la vitesse de prise de vue ou aux changements d'objectif et d'éclairage, surviennent-elles souvent au cours d'une prise. Le travail sur la lumière et sur les contrastes est essentiel: la matière visuelle est composée, sculptée, de sorte que l'image, fluide et changeante, sa surface tantôt obscurcie, tantôt animée comme par les pulsations du hors-champ infiltrant le cadre, oscille entre le figuratif et l'informe. C'est comme si les univers créés par Grandrieux étaient faits d'une multitude de strates de vitesses et d'intensités différentes à travers lesquelles les personnages semblent soit se désintégrer, soit servir de passeurs.

Les orifices, la bouche et le sexe des femmes obsèdent Jean, le tueur de Sombre; c'est pourtant son propre corps qui crée un trou dans la texture du film. Les plans le présentent de préférence de dos, la tête et les épaules formant une masse floue qui masque une partie du cadre. Évoquant le choix du lieu de tournage pour Sombre, les paysages densément boisés de la Haute-Loire, le réalisateur souligne l'importance de la faible variation de luminosité et de couleur entre terre, arbres, et ciel. ${ }^{29}$ En

28. P. Grandrieux, "Un cinéma visionnaire», Le Technicien du film, ${ }^{\circ} 530,2003$, p. 24-28 et particulièrement p. 27.

29. P. Grandrieux, «Entretien avec Philippe Grandrieux», par N. Renaud, S. Rioux, N. L. Rutigliano (1999), Hors Champ, [en ligne], [URL: www.horschamp.qc.ca/Emulsions/ grandrieux.html], consulté le 22 février 20 IO. 
filmant à l'heure où le soleil est bas sur l'horizon, en jouant sur la sousexposition et en diminuant le nombre d'images par seconde, Grandrieux crée une atmosphère d'opacité, où la limite entre avant-plan et arrièreplan ainsi que les contours de la figure humaine deviennent périodiquement indiscernables..$^{\circ}$ À la fin du film, Jean semble finalement s'abandonner à l'attraction de l'informe: dans la dernière séquence, filmée en DV, il s'enfonce toujours plus avant dans un épais sous-bois avant de s'allonger et de laisser son corps se fondre à la matière organique de l'humus qui recouvre le sol forestier.

Il y a, dans la première partie du Pola X (1999) de Léos Carax, un épisode comparable à la séquence énigmatique sur laquelle se clôt Sombre. Le personnage principal, Pierre ${ }^{31}$, est un garçon à la jeunesse dorée, dont l'existence insouciante bascule lorsque surgit le spectre d'une histoire qui lui était jusque-là étrangère. Le passé ressurgit sous les traits d'une demi-sœur, Isabelle, venue des Balkans, née et abandonnée dans un pays en guerre (la Bosnie?). ${ }^{32}$ La scène qui décrit leur première rencontre se déroule dans une atmosphère crépusculaire. Pierre surprend Isabelle à l'orée d'une futaie, et se lance à la poursuite de la jeune femme qui fuit dans la forêt. Très sombres et contrastées, les images donnent presque l'impression de visionner un négatif. Nimbée d'une lueur blafarde, la chair est cadavéreuse. À moitié cachés par sa longue chevelure, les yeux de la jeune femme sont deux cavités obscures. Plus le couple s'enfonce dans le dense réseau végétal du sous-bois, plus les contours s'estompent, et plus les corps semblent absorbés par ce qui les entoure. Dans la suite du film, plus le lien - que l'on pourrait qualifier de vampirique - qui se tisse entre les deux personnages se resserre, plus l'image semble vidée de sa lumière, et plus les corps tendent à fusionner entre eux et avec la texture de l'image.

De même, La Vie nouvelle construit un univers incertain, liminal, où les lumières rasantes dominent - c'est l'heure entre chien et loup dont l'ambivalence est capturée dans les images sous-exposées, les aubes et crépuscules indistincts, ou encore l'obscurité d'un night-club trouée par

30. M. Beugnet, "Cinema of evil: Philippe Grandrieux Sombre and La Vie nouvelle», Studies in French Cinema, vol. 5, nº 3 , 2006, p. 175-184.

3I. Le film s'inspire du roman de H. Melville, Pierre or the Ambiguities (I852).

32. Même en tenant compte du contexte formel spécifique créé par les films cités dans cet article, on doit évidemment s'interroger sur l'imprécision avec laquelle la région des Balkans et de l'ex-Yougoslavie est évoquée. Les personnages féminins, comme représentation de «l'autre», ne sont pas moins problématiques. Sur la notion de Transylvanie dans l'imaginaire contemporain, voir M. Beugnet, Cinema and Sensation, ouvr. cité, p. I44-I49. 
les rais des éclairages artificiels. Les corps et les visages apparaissent rarement dans leur intégralité ; la lumière ou, au contraire, l'obscurité semblent toujours sur le point de restituer les corps à la matière indistincte dont ils sont issus. Plus qu'aucun autre des protagonistes du film, le personnage de la prostituée, Mélania, parait irrémédiablement captif d'un faisceau de regards et de désirs antagonistes au sein desquels elle semble se dissoudre. Figure élusive, elle dessine une silhouette mince et sombre contre le jour blafard qui baigne les couloirs de l'hôtel-bordel, se transformant en un trait de lumière tourbillonnant sur la piste du night-club, avant de se métamorphoser en monstrueuse créature, mi-femme mi-bête, à la fin du film. C'est l'effet synesthétique d'une caméra thermique ${ }^{33}$ qui transforme cette scène, et l'ultime apparition de Mélania, en vision infernale; un magma informe et hurlant emplit le cadre, agglomérat de corps en fusion duquel les figures humaines peinent à s'extirper, yeux et bouches béants comme des cratères noirs.

Dissolution de la forme et de la voix humaine dans la matière filmique; évocation de l'informe et du regard de l'objet qui menacent l'intégrité du corps à l'écran, et désavouent à la fois la notion de sujet autonome et celle d'un monde objectif entièrement soumis à l'emprise du regard subjectif: il n'est pas surprenant que le type de motifs visuels que je viens d'évoquer se retrouvent notamment dans des films qui parlent d'exil. L'exilé, c'est à la fois le «voyant»- pour reprendre le terme de Deleuze -, celui qui se trouve confronté à un monde dont il voit parfois les failles mais dont le sens, souvent, lui échappe; et «l'autre» exotique, incompréhensible ou abject, qui, soumis au regard inquisiteur ou consommateur, se trouve réduit à la condition d'objet.

C'est à cette juxtaposition que l'énigmatique séquence de début de $L a$ Vie nouvelle doit sa force d'évocation: l'œil prédateur de la caméra traversant à toute vitesse un no man's land crépusculaire pour s'écraser contre les regards sidérés d'un groupe de voyageurs égarés dans la nuit. À ces regards de «voyants» (groupe de réfugiés quittant une zone de conflit?) répondra bientôt le regard inerte et terrifiant des «espaces quelconques», «friches», «terrains vagues», "cités en cours de démolition ou de reconstruction», «auxquels nous ne savons plus comment réagir».34

33. Caméra qui enregistre les ondes de chaleur émises par les corps.

34. Dans la préface à l'édition en anglais de L'image-temps, Deleuze résume ainsi le passage de l'image-mouvement à l'image-temps, et du personnage «actant» au personnage «voyant». Si la rupture s'effectue pour Deleuze après la seconde guerre mondiale, les 
L'exil est également au cœur d'Adien (2003) d'Arnaud des Paillières, dans lequel la perte de soi est comparée à l'expérience du deuil. Deux narrations se chevauchent en effet dans Adieu: le récit du voyage d'un immigrant clandestin qui a laissé sa fille en Algérie, et celui d'une famille française d'exploitants agricoles, un père et ses fils, confrontés à la mort d'un fils pour le premier, d'un frère pour les seconds.

"C'est un soir gris, crépusculaire. La route est plongée dans la pénombre.» En voix off, un prêtre lit l'homélie d'une messe d'enterrement. Sur l'écran défilent les images d'un paysage mystérieux filmé en contre-plongée: la terre, une colline sombre qui occupe presque tout le cadre; le ciel, une masse nuageuse à travers laquelle le soleil peine à percer. Comme dans Sombre et La Vie nouvelle, dans Adieu, la lumière est souvent crépusculaire et la limite entre ciel et terre est ténue. Comme les films de Grandrieux, le long métrage de des Paillières crée un champ figural où la forme humaine, silhouette fragile que les longues focales tendent à écraser contre le fond de l'image, est confrontée à la réalité de la matière et se révèle dans sa vulnérabilité. Images floues et tremblées, surimpressions, sous-exposition, grain de l'image et absence de contraste, hétérogénéité et métamorphoses de la bande sonore: les distorsions de l'image et du son manifestent les variations de densité de la «matière» du réel, cette gangue où la forme humaine est captive.

Adieu se tient aux confins de la représentation, dans un espace-limite flirtant avec l'abstraction (la caméra oscille de part et d'autre d'une barre de flou), voire tout bonnement avec l'invisibilité, creux de l'absence où se tient le vrai. Antifigurative, la stratégie d'Adieu s'inscrit dans une logique de décollement visant à extraire l'Être de la gangue de la présence. ${ }^{35}$

Dans cet affrontement entre l'Être et la matière que met en scène des Paillières, la figure du père est emblématique. La mort du fils le transforme en un bloc de refus silencieux, hésitant, comme nous le rappelle

termes de la citation n'en résonnent pas moins avec une remarquable pertinence dans le contexte contemporain des populations déplacées: "The fact is that, in Europe, the postwar period has greatly increased the situations which we no longer know how to react to, in spaces which we no longer know how to describe. These were "any-spaces-whatever", deserted but inhabited, disused warehouses, waste ground, cities in the course of demolition or reconstruction. And in these any-spaces-whatever a new race of characters was stirring, a kind of mutant: they saw rather than acted, they were seers» (G. Deleuze, Cinema 2. The TimeImage, traduction H. Tomlinson et B. Habberjam, Minneapolis, University of Minnesota Press, 1989, p. XI).

35. F. Bégaudeau, «Trop fort (à propos d'Adieu)», Cahiers du cinéma, n' 596, 2004, p. 76-77 et particulièrement p. 77 . 
la voix off, entre «ce qui est vivant, et ce qui devrait l'être». Tandis que se déroulent autour de lui les préparatifs de l'enterrement puis la mise en terre du fils, la silhouette du père fait "tache » : elle forme à l'écran une masse sombre et floue qui obscurcit une partie du cadre. Le chaos envahit alors le «corps» du film: images et sons qui vibrent, perdent leurs contours, se désagrègent, avant de s'évanouir dans le vide de la surexposition. ${ }^{36}$ Le contrechamp pourrait être, ici aussi, le regard de l'inanimé, celui qui s'affirme à l'image avec une évidence effrayante dans la séquence du générique de début. Une série de longs plans-séquences pris dans une usine documentent avec précision le montage d'un poids lourd - celui qui emmènera dans ses flancs l'immigré clandestin dont le parcours se déroule parallèlement à celui de la famille en deuil. La figure humaine a peu de place dans cet environnement quasi automatisé où la machine prend progressivement forme et domine le cadre: corps fragmentés et silhouettes floues, les ouvriers y apparaissent comme de simples éléments épars d'une chaîne d'assemblage bien plus complexe. Et lorsque finalement le poids lourd quitte l'usine pour prendre la route, la cabine du conducteur semble vide: la surface sombre du pare-brise, impénétrable, reflète le paysage.

\section{Un rapport esthétique au monde et aux autres}

S'ils construisent des espaces liminaux où la place de l'humain (corps, personnage, ou simple forme) est toujours incertaine, les réalisateurs que l'on vient de citer ne se complaisent pas pour autant dans un relativisme nihiliste. L'expérience sensuelle et esthétique qu'ils nous offrent à la fois se nourrit de la force destructrice qui habite l'univers de leurs films et la contrebalance.

Est-il nécessaire, d'ailleurs, d'envisager la réduction ou la dissolution de la figure humaine comme la manifestation d'une angoisse existentielle? Vivian Sobchack nous renvoie à Maurice Merleau-Ponty, et nous rappelle qu'une «diminution de la subjectivité» ne signifie pas forcément atrophie perceptuelle et épistémologique. Le regard de l'inanimé n'est pas obligatoirement celui du mauvais oil, la négation du vivant, la confirmation d'un mal-être-au-monde. Faire l'expérience de la fragilité

36. La remarquable scène du cimetière d'Adieu rappelle d'ailleurs la séquence du bombardement d'un cimetière qui sert d'ouverture au film de L. Carax Pola X. 
de l'être-sujet, c'est aussi accéder à un état de conscience accrue de la présence physique du monde extérieur, s'ouvrir à un «rapport esthétique au monde et aux autres ».37

On pense alors à cette séquence, au début du dernier film de Pascale Ferran, Lady Chatterley (2006), où le personnage éponyme se rend pour la première fois seul dans la forêt. La jeune femme porte des vêtements aux teintes automnales. Elle passe le portillon qui sépare le jardin du domaine boisé, et sa silhouette, pressée contre le fond par l'effet de la longue focale, semble fusionner avec la matière végétale saturée de textures et de couleurs.

On pense aussi au singulier film-poème réalisé par Douglas Gordon et Philippe Parreno. Dix-sept caméras pointées sur Zinedine Zidane pendant toute la durée d'un match de football: le résultat aurait pu être le portrait atypique d'une star mondiale ${ }^{38}$, mais c'est tout autre chose qui est proposé au spectateur. Zidane, portrait $d u X X I^{e}$ siècle (2006) est un film dans la lignée d'un Dziga Vertov, entre l'expérimental et le populaire, qui utilise tout ce que les technologies actuelles de l'image et du son ont à offrir pour créer le portrait sensible et captivant d'un monde dominé par l'audiovisuel. Modelé à partir d'images et de sons enregistrés sous tous les angles, Zidane forme une sorte de «corps filmique» que le spectateur est invité à investir: une «façon de voir, de vivre le football autrement, comme de l'intérieur ».39 On est alors tenté de comparer aux battements d'un vaste cœur le martèlement sourd qui vient en vagues des tribunes.

Ce corps filmique est composé d'une multiplicité de textures: images tournées en DV haute définition ou en 35 millimètres format scope, issues d'un satellite ou refilmées sur un écran de télévision; son direct, commentaires télévisés et compositions musicales. Le matériau visuel et sonore présente ainsi une variété de grains et de tonalités qui confère au film (à la fois comme support / surface et comme spectacle / contenu) cette tactilité caractéristique de la vision haptique. Du son isolé au mixage composite, du plan large à l'extrême gros plan, en passant par l'écrasement de la perspective opérée par le téléobjectif, un rapport dynamique s'instaure entre le fond et la forme. Le champ visuel est soumis à une constante fluctuation entre l'abstrait et le figuratif. Tantôt la figure humaine appa-

37. V. Sobchack, Carnal Thoughts, ouvr. cité, p. 286 et p. 289-290.

38. Un projet similaire, prenant G. Best comme sujet, a été tenté par H. Costard en I97I: Fussball wie noch nie.

39. J.-M. Colard, «Zidane. Un portrait du XXI siècle de Douglas Gordon et Philippe Parreno », Les Inrockuptibles, ${ }^{\circ} 547,2006$, p. 54. 
raît dans son intégralité, tantôt elle se dissout dans la texture même de l'image (ce sont les surfaces abstraites, pixellisées qu'offre notamment le générique de début); ou encore elle apparait morcelée ou floue. De même, le champ sonore est une alternance entre des bruits isolés et fugitifs (respiration, soupirs, frottement de semelle sur le gazon), et les clameurs explosives et assourdissantes de la foule. Pris dans cette oscillation entre le distinct et l'indistinct, la forme singulière et l'informe collectif, le corps du joueur est une figure aux contours instables. Soumise au faisceau des regards d'une foule innombrable, elle devient imprécise, elle se dissout. À moins que, l'espace d'un instant, dans le passage du net au flou, elle ne fasse corps avec l'arrière-plan, avec la multitude des spectateurs.

Le film ouvre ainsi un espace de multiples «devenirs ${ }^{4}$ où le corps matériel (individuel ou collectif, sur ou devant l'écran) et le corps comme métaphore ou comme signe (le spectateur, le joueur et la foule comme entités abstraites, constructions psychologiques, socio-économiques ou culturelles) entrent en résonance au sein du corps filmique. Les séquences d'actualités télévisées insérées au milieu du film, parce qu'elles incluent, entr'aperçue parmi les images d'un attentat à Basra, la vision fugace d'un Irakien portant un tee-shirt Zidane, n'apparaissent dès lors plus comme un hors-champ se rappelant à notre bon souvenir, mais comme l'expression d'une irréductible continuité. Cette fois c'est par le montage, en réunissant au sein du film des éléments hétérogènes qui font pourtant partie d'une même matière du réel, que le cinéma évoque cette continuité déjà à l'œuvre dans le jeu de la dissolution de la figure individuelle au sein du tout collectif. ${ }^{41}$

Permettre à la forme humaine de se fondre à la matière ; relier, ne seraitce que fugitivement, la figure distincte à l'informe, c'est en souligner la fragilité, et, par extension, relativiser la notion même de sujet. Mais c'est

40. C'est-à-dire, dans le sens où l'entendent G. Deleuze et F. Guattari, des processus ininterrompus de métamorphose par contiguité: Mille plateaux, ouvr. cité, p. 284.

4I. À propos du cinéma de C. Marker, F. Niney remarque : «L'histoire apparait comme une histoire en cours, non exclusive dans le sens où d'une part le spectateur ne la regarde pas défiler de l'extérieur, d'autre part n'est pas supposée une fin, une seule. » Le cinéma devient alors un moyen de «recoudre, réparer, remailler le fil interrompu du tissu, de l'histoire, de la vie» (F. Niney, «L'éloignement des voix répare en quelque sorte la trop grande proximité des plans", Théorème, $\mathrm{n}^{\circ} 6,2002$, p. IOO-III et particulièrement p. IO8 et p. IO2). Voir aussi M. Beugnet, "Du film d'art à l'art du film: Les statues meurent aussi de Chris Marker et Alain Resnais», CinémAction, ${ }^{\circ}{ }^{\circ}$ 22, 2007, p.39-48. 
aussi manifester la possibilité d'un rapport au monde différent, à savoir un rapport empathique, que le cinéma, mieux que toute autre forme d'expression semble-t-il, sait évoquer. Certains films nous offrent ainsi l'intuition d'un état de flux où la dissolution de l'être dans la matière n'est plus simple anéantissement, mais aussi «extension sensuelle et sensible de nous-mêmes ».42

42. V. Sobchack, Carnal Thoughts, ouvr. cité, p.290. Le terme sensible en anglais renvoie à la fois à la perception sensible et à «sensé». 
Virtuel 



\section{::: \\ L'image ralentie: \\ de la caméra-œil au bullet-time}

Plaçons-nous au centre vivant de ce monde en formation, comme une nébuleuse dont la densité s'accroît et qu'une force irrésistible entraîne, avec une vitesse accélérée, sur les routes du devenir. Décomposons ces éléments mobiles en suivant, pour nous y guider, ce Ralenti qui nous dévoile les reptations musculaires patientes d'un cheval ou d'un chien au galop, la nage lente d'un boxeur ou d'une danseuse dans le fluide atmosphérique, la danse solennelle du vol des oiseaux et des insectes, la caresse ondulante de l'eau que bouleverse la tempête, le méticuleux travail de rupture de la balle de revolver.

Élie Faure, "Introduction à la mystique du cinéma », Fonctions du cinéma.

Tous ses mouvements, cachés ou apparents, l'appareil les accomplit, en effet, pour le compte de l'œil humain. Celui-ci en devient, tantôt comme un oil fixe à facettes, comme un multiple d'yeux, dont chacun possède une perspective particulière; tantôt comme un œil mobile d'escargot, un œil monté sur une tige extensible et rétractile, un œil pouvant recueillir ses informations, non plus toujours à distance plus ou moins fixe, plus ou moins grande, mais aussi au proximum de la visibilité, presque au contact de l'objet, et pouvant maintenir ce contact si l'objet se déplace.

Jean Epstein, «Le monde fluide de l'écran», Écrits sur le cinéma. 
Matrix ${ }^{1}$, la trilogie des frères Wachowski, entend figurer - on n'a pas manqué de le lui reprocher - la réalité virtuelle sous sa forme radicale, celle d'une simulation intégrale et complètement immersive du réel. Nous avons développé ailleurs la question pragmatique qu'instruit cette fable eschatologique. ${ }^{2}$ La résistance opposée par une fraction héroïque de l'humanité à la puissance aliénante de la Matrice prend généralement la forme du «coup de main», tactique bien connue des théoriciens de la guérilla: l'attaque surprise, menée coup sur coup sur deux fronts isolés, mobilise en pratique toutes sortes de dispositifs techniques susceptibles d'accroître la rapidité et l'efficacité de la manœuvre. Comme dans le genre du western ou du film de guerre, il s'agit le plus souvent de trouver la passe, en l'occurrence de se donner une prise sur un univers simulé qui, sans pouvoir être situé nulle part, n'en a pas moins sa géographie et sa topographie propres. La réalité virtuelle a alors moins à voir avec un territoire éthéré ou un mirage collectif émanant de cerveaux en cuve, qu'avec une interface où la possibilité d'entrer et de sortir, autrement dit de se localiser, s'avère le problème principal. Or parmi la débauche d'artifices et d'effets spéciaux qui rendent sensibles la texture du virtuel et les prises qu'elle offre à l'action, un effet de ralenti d'un genre nouveau occupe une place particulière; il propose une solution technique et esthétique remarquablement bien ajustée à une problématique des mondes virtuels que le film aborde de manière beaucoup moins naïve qu'on ne le dit souvent. Popularisé par le film sous l'appellation de «bullet-time» - littéralement, le temps de la balle (de revolver) -, cet effet a le plus souvent échappé à l'attention des commentateurs. Même les plus favorablement disposés n'y ont vu qu'une nouvelle prouesse des computer generated images (images de synthèse) à verser au compte du génie infographique, son principe étant déjà donné par certains films d'animation réalisés quelques années plus tôt.

Aux yeux des tenants de l'esthétique pure ou de l'éthique des images, les «effets spéciaux» conjuguent d'ailleurs plusieurs défauts rédhibitoires: ils

* Cet article reprend un extrait de l'ouvrage d'Elie During Faux raccords : la coexistence des images (C) Actes Sud, 2010).

I. The Matrix, A. et L. Wachowski, Warner Bros., I999; The Matrix Reloaded, 2003; The Matrix Revolutions, 2003.

2. Voir E. During, «Fiction téléphonique et cinématographie virtuelle», Mecanic cinéma. Technologies, machines, outils, objets divers, E. Burdeau éd., Saint-Sulpice-sur-Loire, ACOR, 2003; Matrix, machine philosophique, en collaboration avec A. Badiou et al., Paris, Ellipses, 2003; "Matrix : comment la philosophie peut s'y faire», en collaboration avec P. Maniglier, Revue d'esthétique, $\mathrm{n}^{\circ} 45,2004$; "Is there an exit from "virtual reality" ? Grid and network from Tron to The Matrix ", The Matrix in Theory, M. Diocaretz et S. Herbrechter éd., Amsterdam, Rodopi (Critical Studies), 2006. 
relèvent simultanément de l'artifice technique et du spectaculaire. Si complexe que soit son montage, l'effet spécial a toujours quelque chose d'une ficelle grossière, ou d'un «trucage». On lui pardonnera d'autant moins de chercher à masquer son origine impure en «lissant» l'image au point de la rendre indiscernable de ses doubles numériques. Par ailleurs, comme tout ce qui ressortit à l'« effet», il se situe aux limites de l'expérience esthétique, là où la dimension de contemplation requise par le jugement de goût risque à tout moment de se retourner en simple commotion. Comme le disait Jean Baudrillard à propos de Matrix, si la machinerie spectaculaire de la Matrice ne devait produire qu'un seul film, ce serait bien celui-là.

Certes, mais que la publicité et les vidéoclips se soient vite emparés du procédé holographique de la «rotation figée», dont l'effet bullet-time constitue d'un certain point de vue la reprise et l'amélioration, n'empêche pas que cette technique représente un jalon important, non seulement dans l'histoire de la chronophotographie qui remonte à Eadweard Muybridge et Étienne Marey, mais encore dans l'histoire du ralenti cinématographique depuis ses origines. ${ }^{3}$ Il est vrai que le Cinématographe lui-même, à ses débuts, fut perçu par beaucoup comme une merveille amusante, tout juste digne du divertissement de foire : il n'est décidément pas facile de penser l'effet et ses médiations techniques. Pour autant, il ne suffit pas de renvoyer les puristes à l'impureté originelle de la chose cinématographique, ni d'invoquer le nom de Georges Méliès, pour avoir justifié ou simplement rendu compte de la puissance singulière d'un nouvel effet spécial, du type d'image ou d'affect qu'il véhicule. Les réflexions qui suivent entendent dégager les coordonnées de cette nouvelle image qui prétend capturer le «temps de la balle», en l'inscrivant dans le contexte plus large d'une question rarement traitée pour elle-même, celle des usages et des valeurs du ralenti cinématographique.

\section{Fonctions du ralenti}

Le ralenti a toujours joui, dans l'esthétique du cinéma, d'un statut ambivalent. Pour commencer, sa fonction de dévoilement semble constamment contredite par sa fonction comique. Paul Valéry le relève dans ses

3. Voir F. Albera et al., Arrêt sur image, fragmentation du temps: aux sources de la culture visuelle moderne, Lausanne, Payot, 2002 ; notamment le texte de E. S. Faden, "Chronophotography and the digital image: Whoa... Déjà vu!»,p.343 et suiv. 
Cahiers: "Le ralenti de cinéma est ce qu'il y a de plus irréel. Il consiste à donner par apparence le mouvement d'un mécanisme à n'importe quel mouvement vrai. ${ }^{4}$ On songe, bien entendu, à la caractérisation bergsonienne du procédé de fabrication du comique : appliqué aux mouvements du corps humain, le ralenti revient à plaquer du mécanique sur du vivant, et par là à détruire irrémédiablement tout ce qu'un geste peut contenir de grâce et de liberté. Pourtant, la fonction de révélation du ralenti peut encore opérer jusque dans cet effet de décomposition du mouvement libre, en offrant une sorte de démonstration négative. Valéry précise en effet: "Donc le temps n'est pas homogène. Le mouvement ne peut se ralentir ou s'accélérer sans changer le possible. Le mouvement in abstracto est inexistant» (ibid.). Sur ce point, il est probablement plus proche de Bergson qu'il ne le croit. Car la durée est précisément ce qui ne peut se diviser, se contracter ou s'étendre, sans changer de nature ou de qualité: c'est le propre des multiplicités qualitatives.

Dans un sens proche, Jean Epstein considère le ralenti comme l'opération fondamentale qui permet de manifester, d'une part, le caractère fluent de toute réalité, le flux sous la forme, et d'autre part, l'animation universelle qui défait les partages catégoriels au profit d'un monisme du mouvement compatible avec une hétérogénéité qualitative irréductible des durées. Le réel fluent, en effet, n'est pas homogène, il ne s'identifie pas à la coulée universelle d'un fleuve au cours uniforme, mais à un torrent plein de remous et de tourbillons, parcouru par des courants de vitesse variable. Avant d'examiner de plus près la portée métaphysique - et même cosmologique - que certains auteurs et cinéastes ont voulu conférer au procédé du ralenti, il faut dire un mot, cependant, de ses usages esthétiques ou formels les plus courants. Car il existe bien d'autres moyens de suggérer le caractère fluent de la réalité - la différence intensive des mouvements qui entrent dans une scène suffit souvent à cela ${ }^{5}$, et inversement la signification du ralenti n'a rien d'univoque, de même que les moyens mis en ouvre pour le réaliser.

Le procédé du ralenti s'inscrit d'abord dans le sillage des expériences chronophotographiques de Muybridge et de Marey. Il répond à un souci qui est au cœur de ces travaux - quelles que soient d'ailleurs les diffé-

4. P. Valéry, Cahiers, II, Paris, Gallimard (la Pléiade), 1974, p. 976.

5. "Dans Shanghai Express, la foule d'une gare chinoise, grouillante et agitée, fait mieux ressentir le calme et la force du couple amoureux» (R. Arnheim, «Le mouvement» [1934], Le cinéma est un art, traduction F. Pinel, Paris, L’Arche, I989, p. 190). 
rences d'orientation qui les caractérisent -, celui d'effectuer la décomposition du mouvement tout en conservant quelque chose de ses articulations naturelles, et même de sa continuité. ${ }^{6}$ Mais cette caractérisation est encore trop générale: elle ne dit rien des usages auxquels on le destine, et notamment de la signification esthétique qui lui est du même coup conférée. Or sur ce point il est facile de dégager, par simple induction, quelques fonctions principales du ralenti.

Sa fonction emphatique est la plus manifeste : elle tient à sa capacité à suggérer la puissance et la vitesse d'un geste, ou à magnifier la virtuosité de celui qui l'accomplit. On songe à la grâce aérienne des plongeurs filmés par Leni Riefenstahl, tout comme aux usages variés (analytiques ou expressifs) du ralenti sportif. Une mention particulière doit être réservée à ce qu'il conviendrait d'appeler l'« effet Steve Austin », en référence à une célèbre série télévisée, L'Homme qui valait trois milliards. Cette figure de rhétorique apparaît comme la matérialisation d'un sophisme: les choses qui se déplacent très vite ne peuvent être clairement perçues qu'à la condition d'être ralenties; il faut donc que les choses que l'on voit se déplacer à une allure inhabituellement lente se déplacent en réalité à grande vitesse. Par exemple, un homme qui court au ralenti d'un pas pesant sera conventionnellement tenu pour un corps-cyborg lancé à pleine vitesse (dans ce cas, la bande-son aux accents métalliques participe pleinement de l'effet). Mais le ralenti emphatique peut aussi bien connoter des propriétés qui n'ont rien de spécifiquement mécanique. Il vient alors intensifier la dimension dramatique ou expressive d'une scène, ou bien en surligner la gravité, la solennité. On songe à la sublime scène de duel des Sept Samouraïs (Shichinin no samurai, Akira Kurosawa, 1954): filmée au ralenti, la chute à terre du guerrier blessé à mort, puni pour son audace et sa grossièreté, exhausse la virtuosité du geste de son adversaire. L'effet est d'autant plus efficace que cette passe d'armes fulgurante aura été montrée deux fois de suite, selon un enchainement de plans quasi identiques: une première fois avec des bâtons, une deuxième fois avec de véritables

6. R. Arnheim rappelle que, dans les premiers temps du cinéma, lorsque les opérateurs tournaient encore la manivelle de leur caméra, il n'était pas rare qu'on modifie légèrement les mouvements en les ralentissant et en les accélérant à volonté pour surligner un geste en lui conférant un caractère plus posé, ou au contraire pour lui donner plus de vigueur en le précipitant: «Cette souplesse dans le défilement de la pellicule fut perdue quand apparut le film sonore qui normalisa le nombre d'images exposées chaque seconde. [...] De nos jours, bien sûr, on utilise le ralenti ou l'accéléré en tant que tels, comme des trucages permettant d'effectuer des changements de fréquence significatifs» (ibid., p. 190-I9I). 
sabres... Le ralenti participe ici de ce que les rhétoriciens appellent le style «élevé»: amplification et hyperbole. Dans d'autres cas enfin, il peut remplir une fonction lyrique ou onirique, que les concepts deleuziens permettent de préciser un peu. Le corps suspendu dans sa chute, ravi à luimême, a quelque chose des images flottantes du rêve; comme la rupture des fonctions sensori-motrices prépare l'avènement de l'«image-temps ", le ralenti ouvre à un nouveau régime de l'image aux limites de l'«imageaction», entre l'«image-perception» et l'«image-affection». Les découpages qu'on vient d'indiquer n'ont évidemment rien d'exclusif. Le finale hallucinatoire de Zabriskie Point (Michelangelo Antonioni, 1970), par exemple, participe également de la deuxième et de la troisième fonction: les «ondes de colère » libérées par l'héroïne à la manière d'un châtiment divin sont aussi ce qui engloutit toute la narration dans ce que Gilles Deleuze appellerait une «situation optique et sonore pure».

Revenons cependant au bullet-time, et tâchons d'en comprendre les modalités techniques: on verra qu'elles sont essentielles à la bonne compréhension du fonctionnement de l'image. Car il s'agit bien, en un certain sens, d'une image fonctionnelle, dont la signification s'épuise dans le montage qu'elle opère et rend sensible.

\section{Bullet-time : la capture du mouvement}

L'usage qui est fait dans Matrix du nouveau procédé de ralenti participe simultanément de toutes les dimensions qui viennent d'être évoquées: il s'agit, tout à la fois, de mettre en scène le corps actif d'un maitre des arts martiaux, de suggérer un mouvement purement spirituel de percée des apparences, et de faire sentir la plasticité temporelle d'un univers virtuel où, comme le rappelle un des protagonistes, les lois de la nature peuvent toujours être "courbées». Mais envisageons le procédé pour luimême, tel qu'il s'exemplifie, dans le premier volet de la trilogie, à travers la scène fameuse au cours de laquelle Neo (Keanu Reeves), sur le toit d'un immeuble, parvient à échapper au tir nourri d'un agent de la Matrice en faisant preuve d'une agilité proprement miraculeuse.

Pour réaliser cette scène, il a fallu que le directeur des effets spéciaux, John Gaeta, perfectionne et transforme un procédé holographique dit de «rotation figée » inventé au milieu des années 1990 par une firme française (Buf Incorporated), en y associant le ralenti et des techniques de morphing destinés à fluidifier le mouvement, mais aussi à rendre possibles toutes 
sortes d'étirements ou de dilatations temporels de la scène par interpolation ou interposition d'images. La technique du bullet-time, c'est là ce qui la distingue, permet en effet d'associer à un ultra-ralenti (de l'ordre de I 000 à 2000 images par seconde) toutes les possibilités dynamiques de la prise de vue habituelle : il s'agit alors moins de geler l'image que d'immobiliser le sujet tout en conservant la libre mobilité d'un oil de caméra lancé à grande vitesse. De fait, le bullet-time n'a plus grand-chose à voir, dans sa mise en œuvre technique, avec le ralenti traditionnel. Celui-ci se contente d'exploiter le rapport entre les vitesses d'enregistrement et de projection en projetant moins d'images qu'on en a enregistré dans le même temps, c'est-à-dire en filmant une scène à une vitesse plus grande que d'habitude, pour projeter ensuite les mêmes images à vitesse normale. Dans le cas qui nous occupe, la scène du duel a d'abord été tournée avec des caméras ordinaires. Dans un second temps, elle a été analysée par ordinateur. Un système de repérage laser (laser-targeting system) permet de produire un «patron» numérique des mouvements de caméra virtuels qui formeront la scène finale; ce patron est ensuite matérialisé par la trajectoire que dessinent dans l'espace une centaine d'appareils photographiques disposés en série, et qui enregistreront le sujet simultanément, mais selon différents angles de vue, à des distances et des hauteurs variables correspondant à différentes phases du mouvement de caméra. Une séquence dynamique est ainsi recomposée à partir d'une série d'instantanés, l'ordinateur exécutant les calculs nécessaires pour restaurer la continuité d'une image à l'autre et assurer la fluidité du mouvement d'ensemble (procédé d'interpolation). La technique de recomposition numérique présente deux avantages. D'une part, elle permet une prévisualisation des séquences à partir d'images de synthèse offrant une doublure virtuelle des acteurs en mouvement; d'autre part et surtout, elle autorise une grande souplesse dans le montage final : zooms, effets de travelling, panoramiques, accélérations et ralentissements, tout cela peut être obtenu sans rien perdre en clarté, ce qui serait pratiquement impossible autrement. La lourdeur du dispositif interdit naturellement de tourner ce genre de scènes dans un environnement normal. Pour Matrix, les acteurs ont donc joué les scènes de combat sur un fond d'écrans verts, et les décors ont été entièrement restitués numériquement par une technique d'habillage 3 D.7

7. Sur tout cela, et sur le caractère singulier des images associées au making of, il faut consulter les bonus des DVD de Matrix et de Matrix Reloaded. Voir notre notice sur Matrix Reloaded dans les Cahiers du cinéma, n 585 , décembre 2003. 


\section{L'image-volume}

Ces précisions étaient nécessaires pour bien saisir ce qui est en jeu dans le procédé du bullet-time. Celui-ci permet d'agencer, dans un même plan, deux dimensions qu'on n'avait jamais vu fonctionner ensemble: les mouvements virtuels d'une caméra libre, et la restitution ralentie d'un mouvement réel, en l'occurrence celui d'un corps qui se penche en arrière et se plie selon des angles improbables pour éviter les balles. Il figure ainsi, dans une sorte de diagramme animé où Jean Mitry aurait sans doute vu un exemple de ce qu'il appelle «image totale $»^{8}$, la perception hallucinatoire d'une balle de pistolet tirée à bout portant, fendant l'air au ralenti.

Ce mouvement-là, celui de la balle, est le premier aspect, le moins intéressant à vrai dire, des scènes réalisées grâce au bullet-time: ce qu'il fait voir s'apparente en ce sens aux procédés les plus classiques du ralenti ou de l'ultra-ralenti. Le déplacement de la balle évoque le vol d'un lourd frelon ou la lente propagation d'une perturbation dans un milieu élastique. Mais cette dimension du procédé est finalement tout à fait secondaire: elle est d'ailleurs des plus simples à obtenir aujourd'hui par les moyens de l'infographie ou de la "cinématographie virtuelle».

L'effet devient réellement intéressant lorsqu'on envisage cette scène d'un tout autre point de vue, qui ne se donne justement plus du tout comme un point de vue, qui fait plutôt glisser l'image sur elle-même, en y introduisant une superposition de durées. Le personnage, qui, tout en manifestant par ce tour de force son élection, parvient à éviter les balles tirées à bout portant, apparaît comme gelé, saisi dans une durée épaisse et infiniment dilatée; il tombe en arrière pendant de longues secondes, comme suspendu dans le vide, tandis que l'œil de la caméra tourne autour de lui à grande vitesse en décrivant des arabesques.

Ainsi, le plan semble tourner tout entier sur lui-même, comme une toupie. Le mouvement de la caméra introduit dans le plan une seconde perspective, en décrochage par rapport à la première : ce n'est plus le jeu

8. L'image totale apparaît chez Mitry comme une variété de l'image "semi-subjective»: en épousant le point de vue d'un personnage qui, tout en étant objectivement donné, occupe une place centrale dans le cadre, elle conjugue, dans un même plan, les caractères de l'image «descriptive» (par ce qu'elle montre), de l'image "analytique» ou subjective (suggérant la vision du personnage), et de l'image «symbolique » (dont la composition manifeste l'intention expressive de l'auteur). Voir J. Mitry, Esthétique et psychologie du cinéma, Paris, Éditions universitaires, I990, p.300. 
du champ/contrechamp, du regardé/regardant qui organise la distribution des points de vue, mais un mouvement virtuel sans mobile identifiable, celui de la caméra elle-même, inscrivant dans le champ un différentiel de vitesses qui ne passe plus entre différents éléments ou sujets en mouvement dans la scène, mais, si l'on veut dire, entre la forme et le contenu de l'image. De sorte qu'on pourrait penser que le bullet-time nous livre une espèce de ralenti au second degré: une image du ralenti, plutôt qu'une image ralentie ou un ralenti de l'image. Tout se passe en effet comme si un ralenti se trouvait ici refilmé à grande vitesse, sans cesser d'apparaître comme ralenti. Or quelle que soit la manière dont on la décrive, image-relief ou image-volume, la chorégraphie numérisée introduit au cour de l'image une dimension de réflexivité qui constitue une saillance dans l'enchaînement diégétique des actions. Traitée par l'œil de caméra qui la cerne sous toutes ses faces, l'image se donne au spectateur comme l'image d'une image.

Un acteur amorce un mouvement à vitesse normale, se trouve un moment comme suspendu en l'air, avant de porter un coup fulgurant et de retomber sur ses pieds. Le «kick» de Trinity dans les premières minutes de Matrix offre un bon exemple de ce qui n'avait jusque-là d'existence que dans les dessins animés adaptés des mangas. De telles scènes ne seraient pas possibles si la technique désignée par Gaeta par le terme «Flo Mo» ne permettait de dilater ou de contracter à volonté la trame temporelle en suggérant contradictoirement le flux continu de l'action et sa suspension, sa concentration extrême en un point privilégié de la courbe d'un geste, sur lequel le regard peut s'attarder et appuyer un instant avant de glisser à nouveau. Cela a fait dire à certains que l'interpolation est avant tout un art de l'interstice - entre deux images, entre deux degrés de vitesse. ${ }^{9}$ Quoi qu'il en soit, avec le bullet-time, le cinéma peut littéralement jouer avec les effets de dilatation ou de contraction de la durée, en faisant sentir de manière concrète les manipulations auxquelles se prêtent les images «en relief». Il y aurait beaucoup à dire sur tout ce qui apparente ce genre de scènes à l'univers du jeu vidéo, et par là même

9. C'est la thèse défendue par l'écrivain de science-fiction et philosophe A. Roberts dans un texte consacré à la question, "The Matrix and Deleuze: bullet-time and betweentime", et dont ne subsiste plus qu'une traduction en allemand sur le site Epilog, [en ligne], [URL: http://www.epilog.de/PersData/R/Roberts_Adam_I965/Interpretationen_SF/O3_Matrix_ACO56.htm], consulté le 23 février 20IO. Roberts est également l'auteur, sous le pseudonyme de Robertski Brothers, d'un livre parodique, The McAtrix Derided, Londres, Gollancz, 2005. 
à la problématique de la «prise» ou de l'interactivité. Lorsque Neo se courbe pour éviter les balles, il est suspendu au-dessus du sol, comme un personnage de jeu vidéo: le ralenti qui décompose le geste rend sensible un raccord, un point de connexion virtuel; par là même il fait voir, en sollicitant les réflexes sensori-moteurs du spectateur-gamer, que la réalité virtuelle (le «simulacre», comme on disait naguère) n'a rien d'un milieu homogène dans lequel il suffirait de s'immerger passivement, mais qu'elle est au contraire éminemment opérable. Multiplement stratifiée, offrant d'innombrables points de contact et d'insertion, elle est une extension, une amplification ou une complexification de la réalité, plutôt que la doublure spectrale qu'on s'imagine trop souvent.

\section{Le virtuel ressaisi en durée}

Il faut néanmoins aller plus loin, et ne pas abandonner trop vite la question du point de vue au profit d'une pragmatique de la prise. Car en vérité le différentiel de mouvement inscrit au cœur du bullet-time est luimême l'indice de durées hétérogènes et pourtant comme tendues l'une vers l'autre, susceptibles de se rejoindre dans une perception d'un genre nouveau. L'image totale combine les vertus de l'image subjective (ou semi-subjective) et celles de l'image descriptive: d'un côté, la durée de la matière et du mouvement purement mécanique de la balle, de l'autre celle du corps-esprit du protagoniste rompu aux arts martiaux et acclimaté au caractère élastique et fluide de la Matrice. Le procédé du bullettime suggère à sa manière un mouvement de dilatation purement mental, celui de l'esprit touchant à la durée-limite de l'instantané, ou, ce qui revient au même, de la pure répétition mécanique. Le ralenti, on l'a rappelé, a souvent eu pour fonction d'évoquer l'extrême vitesse. Par une sorte de passage à la limite, il allégorise ici la capacité de l'esprit attentif d'égaler asymptotiquement, à la faveur du geste le plus rapide et le plus précis, une durée infiniment détendue. ${ }^{10}$

En somme, ce que suggère l'effet mis en ouvre par les réalisateurs de Matrix, c'est moins la vitesse elle-même qu'un certain rapport entre deux vitesses ou régimes de durée; moins la prouesse physique que le devenir

IO. On raconte que le fondateur des arts martiaux, Morihei Ueshiba, avait lui aussi le don étrange d'éviter les balles par de simples rotations du corps et de la tête. "L'esprit calme", disait-il, est "comme la pointe immobile d'une toupie». Voir K. Ueshiba, The Spirit of Aikido, Tokyo, Kodansha International, 1984. 
qui porte Neo aux limites d' «une durée de plus en plus éparpillée, dont les palpitations plus rapides que les nôtres, divisant notre sensation simple, en diluent la qualité en quantité ». ${ }^{\text {II }}$ Le problème de la simulation et de la réalité virtuelle y gagne une détermination plus précise et mieux ajustée: le spectacle des anamorphoses temporelles envisage en effet la simulation à partir d'une expérience singulière du temps ou de la durée, plutôt que du rapport encore trop extérieur - trop spatial en somme - entre l'original et la copie, la chose matérielle étendue dans l'espace et sa représentation immatérielle dans l'esprit, sujet d'une «hallucination collective» ou «consensuelle», pour reprendre l'expression de William Gibson dans Neuromancer. ${ }^{12}$

Mais laissons de côté, pour le moment, les conséquences métaphysiques qui peuvent se déduire d'un tel changement de perspective. La coexistence, dans un même plan, d'un ralenti traditionnel - quoique numériquement composé - et d'un mouvement apparent de caméra lui aussi numériquement recomposé - nous paraît en soi une innovation remarquable. Bien entendu, que rien de tel n'ait été tenté auparavant, même dans le procédé de la rotation figée, ne garantit nullement que soit produite, du même coup, une «idée» de cinéma (une «idée en cinéma», comme Deleuze aimait à dire). Il ne suffit pas d'avoir reconnu un tour de force technique pour s'être donné les moyens d'une évaluation esthétique, et le bullet-time fournit l'occasion d'appliquer la maxime pragmatique rappelée par Stanley Cavell: «Rien ne constitue la "possibilité d'un moyen d'expression" à moins que son utilisation ne lui donne signification et importance », ce qui signifie que «la seule façon de divulguer une "possibilité d'un moyen d'expression", ce sont des œuvres réussies qui définissent ses vecteurs . $^{13}$

Il nous semble que Matrix est une œuvre réussie dans son ordre, parce que sur bien des points elle invente des solutions qui répondent exactement à ses intentions générales, et aux problèmes plus particuliers qu'elle entend formuler: cela n'en fait certes pas un chef-d'œuvre, ni même un «grand film» qu'un cinéphile pourrait vouloir faire figurer dans son panthéon. Il faut pourtant bien lui reconnaître une efficace singulière,

I I. H. Bergson, La Pensée et le mouvant [1934], Paris, PUF (Quadrige), I990, p. 2 IO.

I2. W. Gibson, Neuromancer, New York, Ace Books, I983. Nous reprenons ici de manière condensée nos analyses développées dans «Trois figures de la simulation », A. Badiou et al., Matrix, machine philosophique, ouvr. cité.

I3. S. Cavell, La Projection du monde. Réflexions sur l'ontologie du cinéma, traduction C. Fournier, Paris, Belin, I999, p. I80. Voir les passages du chapitre XVIII consacrés à l'arrêt sur image et au ralenti. 
et ce n'est pas en évoquant les effets spéciaux en général, ou les vertiges du Spectacle, qu'on aura commencé à en élucider les ressorts. La portée esthétique du procédé de ralenti particulier que désigne le bullet-time ne peut réellement être dégagée que dans le contexte filmique où elle s'inscrit. Or s'il n'est pas question de tirer directement des dimensions proprement techniques d'un tel procédé des conclusions de nature ontologique ou esthétique, il est difficile de faire abstraction, lorsqu'il s'agit d'en dégager la signification, des usages et des discours qui ont perçu dans le ralenti un enjeu stratégique pour la définition des puissances du cinéma en général. Élie Faure et Jean Epstein, parmi d'autres, ont vu dans le procédé du ralenti une ressource essentielle, à tel point qu'il apparaît chez eux comme l'exemple privilégié qui permet d'illustrer la révolution introduite par le cinéma dans notre rapport à la réalité. Mais puisque le ralenti semble être d'abord lié aux moyens mis en œuvre pour la capture du mouvement et sa restitution, il n'est pas inutile de commencer par se pencher sur les analyses d'André Bazin : c'est à partir de lui, en effet, que peut s'opérer le partage des eaux entre deux conceptions du ralenti, le ralenti ontologique et le ralenti constructiviste. Ce détour par un de nos grands classiques permettra, au point de recoupement de ces deux orientations, de préciser la portée de l'innovation introduite dans le domaine des effets de ralenti par la technique du bullet-time.

\section{Bazin et le paradoxe du ralenti naturel}

Bazin en a fait le nerf de sa lecture : l'enregistrement automatique du réel est capable de libérer et de rendre sensibles des virtualités objectives dans la trame même du réel, comme aucun autre art n'avait pu le faire avant le cinéma. Les premières pages de Qu'est-ce que le cinéma? sont connues:

Le reflet dans le trottoir mouillé, ce geste d'un enfant, il ne dépendait pas de moi de les distinguer dans le tissu du monde extérieur; seule l'impassibilité de l'objectif, en dépouillant l'objet des habitudes et des préjugés de toute la crasse spirituelle dont l'enrobait ma perception, pouvait le rendre vierge à mon attention et partant à mon amour. ${ }^{14}$

Dans un geste dont Jacques Rancière a bien identifié l'ascendance romantique ${ }^{\mathrm{r}}$, la puissance de révélation de l'objectif impassible, l'essen-

I4. A. Bazin, Qu'est-ce que le cinéma? Paris, Cerf, I975, p. I6.

I 5. Voir notamment J. Rancière, La Fable cinématographique, Paris, Seuil, $200 \mathrm{I}$. 
tielle passivité que semble impliquer son automatisme se conjuguent fort bien avec la puissance opérante du montage dès lors que ce dernier prolonge l'œuvre d'enregistrement du réel qui, comme l'a dit Bazin, a pour premier office de produire une espèce de moulage ou d'empreinte de la durée. Cette conjonction des contraires, passivité de l'enregistrement et activité organisatrice des formes, est tout particulièrement sensible dans le jeu qu'autorise le décrochage entre le temps de la prise et le temps de la projection, à condition toutefois que le montage affiche nettement ce décrochage et ne recoure pas au procédé de la prise accélérée. En effet, s'il continue à faire fond sur la matière que lui offre la prise «naturelle » à 24 images par seconde, le ralenti, qui est une forme élémentaire du «trucage », n'a pourtant rien d'une "falsification » du réel - qu'il faudrait alors envisager dans un rapport d'opposition au naturalisme spontané de l'image cinématographique : il participe pleinement, si l'on suit Bazin, de la puissance de révélation de l'enregistrement. Aux images «gonflées» jusqu'à l'enflure, il faut toujours préférer la sobriété des images montées, car loin de dénaturer la durée réelle des choses capturée par l'impassible objectif, l'opération du montage la confirme et l'accomplit.

Ainsi, dans Le Mystère Picasso de Henri Georges Clouzot (1956), lorsqu'il s'agit de filmer le peintre au travail, l'accélération ou le ralenti s'obtiennent simplement par augmentation ou par réduction, c'est-à-dire par insertion de plans intermédiaires, ou au contraire par le procédé de l'ellipse qui fait disparaître, à la faveur de quelques coupes adroites, les temps morts ou les durées trop longues. ${ }^{16}$ À aucun moment, cependant, le réalisateur n'accélère le temps «en soi », celui de la peinture se faisant, dont le montage nous donne l'illusion d'une saisie sur le vif, à travers ces gestes à l'état naissant qui évoquent la «germination» et le «bourgeonnement» des lignes et des couleurs dans le dessin d'animation d'Oskar

I6. A. Bazin, Qu'est-ce que le cinéma ? ouvr. cité, p. 198. Cette pratique du ralenti est aussi celle qu'envisage J. Mitry dans les développements subtils qu'il consacre au rythme cinématographique et au montage (Esthétique et psychologie du cinéma, ouvr. cité, p. I55 et suiv.). C'est l'occasion de rappeler que l'origine du montage, selon Mitry, réside dans la possibilité de montrer «une action plus longue en réalité que le temps de projection du film», autrement dit de produire une version condensée - et en ce sens accélérée - de l'action par les seules ressources du montage (ibid., p. IO2). La Vie d'un pompier d'E. S. Porter en fournit la première illustration en montrant le déroulement d'une action à travers une série de raccourcis, sans avoir à accélérer la durée d'aucune des séquences. Le film fut réalisé en 1902, un an avant The Great Train Robbery. Mitry ne juge pas utile de considérer la possibilité inverse, celle qui consiste à faire «gonfler» l'action en y insérant des plans qui suspendraient, retarderaient et ralentiraient le cours de l'action réelle. Quant au procédé du ralenti «mécanique», il n’est, sauf erreur, pas même mentionné. 
Fischinger ou de Norman McLaren. Par une forme de paradoxe analogue à celui du comédien de Diderot, c'est en s'assumant pleinement comme montage que le ralenti cinématographique peut échapper au reproche de l'artifice et trouver sa place dans ce grand «film bergsonien» consacré à Picasso:

[I]l faut distinguer radicalement le temps du montage et celui de la prise de vue. Le premier est abstrait, intellectuel, imaginaire, spectaculaire, le second seul est concret. Tout le cinéma est fondé sur le libre morcellement du temps par le montage, mais chaque fragment de la mosaïque conserve la structure temporelle réaliste des 24 images seconde. Clouzot s'est bien gardé - et on ne saurait trop l'en féliciter - de nous faire le coup du tableau-fleur, s'épanouissant comme les végétations des films scientifiques à l'accéléré. (Ibid., p. 200)

Ainsi Clouzot comprend «la nécessité d'un temps spectaculaire, utilisant à ses fins la durée concrète sans, pourtant, la dénaturer». Car le cinéma n'est pas «simple photographie mobile d'une réalité préalable et extérieure », il est «organisé en symbiose esthétique avec l'événement pictural» (ibid.).

Le cas du Mystère Picasso fait bien apparaitre le problème que pose, aux yeux de Bazin, le ralenti traditionnel ou «mécanique» - celui qui s'obtient en projetant moins d'images en un temps donné qu'on en a enregistré dans le même temps. Avec le différentiel de vitesses entre la prise et la projection, tout se passe en effet comme si la prise elle-même se trouvait contaminée par un procédé de montage, selon une manœuvre frauduleuse que contribuent à masquer les conditions psychophysiologiques de la perception. Car celle-ci continue, quant à elle, à réclamer 24 images par seconde. Le ralenti perturbe l'ancrage «naturel» du temps du montage dans le temps de la prise, parce qu'il brouille la différence entre ces deux niveaux. Aussi, la reconnaissance du ralenti comme moyen de révélation du réel, en «symbiose esthétique » avec la durée des choses mêmes, passe par la remise en question de l'axiome - bien peu bergsonien, en vérité - qui guide d'un bout à l'autre l'analyse de Bazin, à savoir que la caméra-œil serait le substitut mécanique d'un œil humain, autrement dit d'un œil naturellement accordé à des durées homogènes à celle de la conscience du spectateur ordinaire. L’usage esthétique du ralenti «mécanique », jouant du différentiel de vitesses entre le temps de l'enregistrement et le temps de la projection, est contemporaine de l'avènement d'un œil non humain fouillant le cœur des choses. Mais il est lui-même partagé entre plusieurs régimes de l'image cinématographique, et c'est ici que les choses se compliquent. On peut en effet distinguer un ralenti construc- 
tiviste, dont les expériences de Dziga Vertov fourniraient le type, et un ralenti ontologique, dont Epstein a donné l'exemple en même temps qu'il en a produit la théorie.

\section{Vertov : le ralenti constructiviste}

L'usage du ralenti dans L'Homme à la caméra (Человекс киноаппаратом, I928) offre l'exemple d'une perception prise pour ainsi dire dans les plis de la réalité. Il faut à cet égard distinguer deux opérations singulières. D'une part, les arrêts sur image fixent à travers une série de photogrammes des gestes qui sont l'analogon des opérations du cinéma lui-même - ceux qu'accomplit l'homme à la caméra au moment de la prise, ou ceux de la monteuse affairée à sa table de montage. L'arrêt et la reprise, qui sont en quelque sorte des cas limites de l'accélération et du ralenti - supposant l'un et l'autre un différentiel de vitesse infini -, laissent affleurer le processus formel du film, en même temps qu'ils allégorisent le réveil de la population d'une ville qui est son sujet explicite. Dans un autre passage où Vertov célèbre à sa façon le corps sportif, le montage fait alterner des plans au ralenti d'un match de football et des plans à vitesse normale décrivant les spectateurs assis dans les gradins. Déliés de la pesanteur, les gestes dessinent de pures lignes de vitesse. Mais ce qui compte plus encore que l'effet d'amplification produit par le ralenti, ce sont justement les différentiels de vitesse entre les deux sortes de plans. Il est d'ailleurs frappant de constater, au sein même des plans ralentis, que l'action se concentre autour des gestes d'interception et de relance - de réception et de passe -, autrement dit sur les enchaînements, les raccords, les intervalles ou les différences qui existent chaque fois entre deux mouvements, deux rythmes, deux flux de durée hétérogènes. Le ralenti magnifie moins la force ou la dextérité des athlètes que le caractère stratifié de la durée du jeu, qui n'est fait que d'accélérations, de reprises, de pointes d'intensité, etc. Le ralenti ne se contente pas d'altérer uniformément le cours du temps en transgressant la ressemblance iconique qu'assure le régime des 24 images par seconde: il rend visible la variation comme telle, qui est une modulation de la durée. C'est ainsi que Vertov parvient à saisir l'élément génétique de la perception. On sait de quelle manière Deleuze a pu lire dans le «ciné-œeil» de Vertov l'avènement d'une «image-perception » d'un genre nouveau, «pure vision d'un œil non humain, d'un œil qui serait dans les choses» : 
Ce que fait le montage, selon Vertov, c'est porter la perception dans les choses, mettre la perception dans la matière, de telle façon que n'importe quel point de l'espace perçoive lui-même tous les points sur lesquels il agit ou qui agissent sur lui, aussi loin que s'étendent ces actions et ces réactions. ${ }^{17}$

Pour voir cela dans L'Homme à la caméra, il fallait évidemment avoir opéré la conjonction avec le premier chapitre du livre de Bergson, Matière et mémoire ${ }^{18}$, et l'idée matérialiste d'un «en-soi de l'image» qui ne devrait plus rien au centrage du champ sur le sujet phénoménologique de la perception. Mais le point le plus important est que la possibilité du montage est alors ordonnée à un mouvement impersonnel - celui de la caméraœil - qui s'affirme partout à travers les «intervalles» entre les mouvements, entre les images. ${ }^{19}$ Ce que cherche Vertov dans les ralentis et les accélérés, c'est «la différentielle du mouvement physique», «le point où le mouvement s'arrête, et, s'arrêtant, va pouvoir s'inverser, s'accélérer, se ralentir ». ${ }^{20}$ Le ralenti comme point d'inflexion marque moins un changement de vitesse (point de vue extensif) qu'un changement d'accélération, une transformation du régime des vitesses (point de vue intensif).

C'est de cette manière que le ralenti constructiviste parvient à dégager de pures lignes de vitesse au sein même de la matière : au-delà de l'action et de la réaction des corps les uns sur les autres, au-delà du mouvement local perçu comme qualité variable attachée à un mobile, c'est toute une énergétique que révèle l'usage du ralenti par le truchement des mouvements virtuels de la caméra-œil. En ce sens, le suspens introduit dans les scènes de combat de Matrix par le procédé du bullet-time peut encore apparaître comme une forme dégénérée du ralenti constructiviste, à ceci près que le caractère différentiel du ralenti se déplace : l'effet bullet-time organise comme on l'a dit la disjonction entre la durée du sujet ralenti et la rapidité de mouvements de caméra qui semblent échapper à l'épaississement général de la durée des corps, de sorte que le différentiel passe maintenant entre la vitesse virtuelle du plan lui-même - où Deleuze voit justement le nerf de l'image-mouvement - et les vitesses figurées par les corps qui le traversent. Cette première virtualisation des corps se double d'ailleurs d'une autre, dont la portée est bien différente. Car si l'on suit la

I7. G. Deleuze, L'Image-mouvement, Paris, Minuit, I983, p. II7.

I8. H. Bergson, Matière et mémoire [I896], Paris, PUF (Quadrige), 2008.

I9. Voir F. Zourabichvili, «The eye of montage: Dziga Vertov and Bergsonian materialism », The Brain in the Screen. Deleuze and the Philosophy of Cinema, G. Flaxman éd., Minneapolis, University of Minnesota Press, 2000, p. I45 et suiv.

20. G. Deleuze, L'Image-mouvement, ouvr. cité, p. I2I et p. I2O. 
fable, les corps virtualisés sont eux-mêmes d'abord des corps simulés, insérés dans la trame d'un monde virtuel. La texture de la Matrice que les coulées de «code vert» allégorisent naïvement doit aussi s'inscrire sensiblement dans les corps en mouvement, au-delà des cabrioles auxquelles nous ont depuis longtemps habitué les films de kung-fu. Ici le ralenti soutient une autre lecture, ontologique, dont Epstein a été l'éloquent défenseur.

\section{Epstein : le ralenti ontologique}

On a évoqué plus haut l'office fondamental que le ralenti remplit aux yeux d'Epstein: il s'agit, par les moyens mêmes de la machine, en suivant la logique d'un «nouvel empirisme», d'échapper aux automatismes de l'intelligence et de la perception ordinaire pour conquérir la vision nouvelle et plus malléable d'une réalité en devenir, tissée de durées diversement rythmées. Le grossissement de la trame temporelle équivaut d'abord à une suppression de l'action, ou de la diégèse, au profit d'un drame intime qui échappe aux ressorts psychologiques habituels du théâtre filmé. Il ne s'agit plus, comme dans le ralenti emphatique dont participe encore le bullettime, de surligner ou de ponctuer l'action, mais de livrer à la perception des devenirs imperceptibles et des micro-durées. Il s'agit de faire affleurer le grain de la durée, avec tous les changements qualitatifs qu'elle implique.

La forme elle-même apparait alors comme une modulation du temps lui-même, ou la limite vers laquelle tend un mouvement infiniment ralenti: «[L]a forme, perpétuellement mobile, comme liquéfiée, n'est plus qu'une certaine lenteur d'écoulement. $»^{21}$ Sous les corps ordonnés à la division des genres et aux règnes de la nature, c'est le grouillement des devenirs, le «monde fluide de l'écran»:

Les dunes rampent; les minéraux fleurissent et se reproduisent; les animaux s'engluent en eux-mêmes et se pétrifient; les plantes gesticulent et expérimentent vers la lumière; l'eau colle; les nuages cassent. ${ }^{22}$

Ce mobilisme universel, qu'Epstein assume pleinement comme un monisme spiritualiste $^{23}$, ne doit pourtant pas faire oublier l'essentiel: le monisme est

2 I. J. Epstein, «Le cinéma du diable» [1947], Écrits sur le cinéma, Paris, Seghers, I975, t. I, p. 348 .

22. J. Epstein, «Le monde fluide de l'écran» [1950], ibid., t. II, p. I49.

23. "Tous les systèmes compartimentés de la nature se trouvent désarticulés. Il ne reste plus qu'un règne: la vie» («L'intelligence d'une machine» [1946], ibid., t. I, p. 255). 
un pluralisme, comme aimait le rappeler Deleuze après William James, et la durée est ce qui diffère sans cesse de soi. Le ralenti ontologique dévoile que le temps «n'a pas de valeur absolue, qu'il est une échelle de dimensions variables». Le ralenti produit «des variations de durée dans la durée même»; il inscrit «un mouvement dans un autre mouvement, un temps dans un autre temps ${ }^{24}$, et c'est cette hétérogénéité, bien plus que la fluidité elle-même, qui est le moteur principal de la nouvelle "photogénie». Sur ce point le ralenti ontologique rejoint le principe opérant du ralenti constructiviste.

Ainsi, la mer agitée au ralenti par le souffle du tempestaire apparaît comme un condensé de mouvements contraires: reconduite à l'élément visqueux, intermédiaire entre l'eau et la glace, le liquide et le solide, elle donne l'image d'une matière nouvelle, un «univers embourbé en luimême ${ }^{25}$, écrit Epstein, mais sourdement travaillé par les lignes de force qui commandent ses métamorphoses. La puissance du virtuel s'annonce dans cette "multiplication du réel » qui fait coexister les hétérogènes dans un système de relations mouvantes. Pour la mettre en œuvre, il n'est même pas nécessaire que le ralenti affiche clairement son propre procédé; il peut opérer de manière imperceptible et rigoureusement non spectaculaire, à l'image du visage lentement décomposé de Marguerite Gance dans La Chute de la maison Usher (1928):

J'ai négligé volontairement au cours de La Chute de la maison Usher tous les effets plastiques que pouvait permettre l'ultra-cinématographe. Je n'ai cherché - si j'ose m'exprimer aussi prétentieusement - que l'ultra-drame. À aucun moment du film, le spectateur ne pourra reconnaitre: Ceci est du ralenti. ${ }^{26}$

On ne peut certes pas en dire autant de Matrix, et ces remarques trop rapides permettent déjà de mesurer l'écart qui sépare les scènes conçues par le moyen du bullet-time et celles qui répondent à la rêverie éléatique d'Epstein. Il n'empêche que le film des frères Wachowski est parcouru par le souci de mettre les formes en variation pour faire toucher du doigt quelque chose de ce «monde fluide» de la Matrice. Mais comme il s'agit surtout de rendre sensible la texture élastique de la Matrice, pour faire

24. J. Epstein, «Le cinéma du diable», art. cité, p. 370.

25. Ibid., p.370. Voir P. Dubois, «La tempête et la matière-temps, ou le sublime et le figural dans l'œuvre de J. Epstein", Jean Epstein: cinéaste, poète, philosophe, J. Aumont éd., Paris, Cinémathèque française, I998.

26. J. Epstein, «L'âme au ralenti» [1928], Écrits sur le cinéma, ouvr. cité, t. I, p. I9I. 
écho en quelque sorte à la souplesse des corps rompus aux arts martiaux, les réalisateurs font onduler les décors, soumettent les corps et la durée même de leurs gestes à des déformations plus ou moins spectaculaires. Plutôt que d'introduire dans l'image ce tremblement imperceptible qui finit par défaire l'expression d'un visage ${ }^{27}$, il s'agit alors d'exhiber directement - et même de manière littérale, lorsque le macadam se plisse sous les pieds du héros - la réalité dans toute sa puissance plastique, pour figurer - là aussi très littéralement - que le corps peut se plier aux lois «courbes» du virtuel. À la décorporation insensible du personnage dont Epstein dévoile «l'âme au ralenti » répond ainsi l'assomption matricielle des corps glorieux nimbés de lumière dorée: deux versions du spiritualisme.

\section{Conclusion}

Cependant, cet univers fluide dans lequel les corps apprennent à couler leurs gestes doit en même temps être intégralement opérable, puisque dans le cas de la simulation, le virtuel est aussi bien d'essence algorithmique. Il est donc naturel que le procédé du bullet-time conjugue les deux faces - constructiviste et ontologique - du ralenti.

Il n'est certes pas question de donner, dans une vue objective, un équivalent plastique de la perception de l'œil non humain logé dans les plis de la matière en mouvement: cette vision qui correspondrait, dans le vocable deleuzien, à la rupture des schèmes sensori-moteurs, demeure interdite tant qu'il s'agit d'un film d'action, ordonné aux exigences de la prise. Il faut néanmoins en donner l'idée, fût-ce de manière littérale, et c'est précisément ce que réalise emphatiquement l'image-volume livrée par le bullet-time. La balle qui fend l'air au ralenti ne nous apprend par ellemême rien de spécial. Il n'est d'ailleurs pas question d'aller «plus vite» que la balle, ou même d'égaler son mouvement sur un plan purement physique, mais seulement de coïncider avec sa durée propre, qui en l'occurrence est

27. "Je ne connais rien de plus absolument émouvant qu'au ralenti un visage se délivrant d'une expression. Toute une préparation d'abord, une lente fièvre, dont on ne sait s'il faut la comparer à une incubation morbide, à une maturité progressive ou, plus grossièrement, à une grossesse. Enfin tout cet effort déborde, rompt la rigidité d'un muscle. Une contagion de mouvements anime le visage. L'aile des cils et la houppe du menton battent de même. Et quand les lèvres se séparent enfin pour indiquer le cri, nous avons assisté à toute sa longue et magnifique aurore. Un tel pouvoir de séparation du sur-œil mécanique et optique fait apparaître clairement la relativité du temps. Il est donc vrai que des secondes durent des heures!» (ibid.) 
infiniment plus «lente», c'est-à-dire moins contractée, que celle de l'esprit concentré. Le projectile de métal qui se déplace d'instant en instant suivant les lois de la mécanique n'est que pure répétition dans l'homogène: l'esprit tendu vers un effort d'intuition sera toujours plus «rapide» que lui. Sur ce point, un bergsonien peut bien s'entendre avec un maitre de kung-fu. Or - et c'est le plus important à nos yeux - le bullet-time suggère l'idée d'une telle expérience perceptive plutôt qu'il ne nous la livre in concreto. Le rapport différentiel des durées - celle de l'esprit, celle de la matière - propose la figuration symbolique d'une expérience perceptive de l'imperceptible qui demeure étroitement liée à l'enchaînement des actions et que nous pouvons seulement supposer être accessible aux protagonistes de la fable, sans y être conviés nous-mêmes. Mais le ralenti chorégraphique indique encore que la simulation se donne comme une expérience intégralement programmée, et donc reprogrammable, comme on le dirait d'un jeu vidéo. Derrière la texture irisée de la Matrice, il y a toute la syntaxe d'un univers digital qui est offert à la main autant qu'à l'œil, et c'est pourquoi le virtuel se donne aussi comme un montage ou un remontage permanent de ses propres effets. Le bullet-time pourrait bien ouvrir en ce sens l'âge d'un nouveau maniérisme, au croisement du jeu vidéo et du cinéma. Que le ralenti y trouve sa place et peut-être de nouvelles puissances est à cet égard une bonne nouvelle: ce peut être l'indice que la manipulation virtuose des images digitales ne nous condamne pas à cette frénésie d'une «crise d'épilepsie minutieusement contrôlée » ${ }^{28}$ que Deleuze croyait déceler dans l'esthétique ordinaire du vidéoclip, et plus généralement dans ce nouveau régime des images sans fond, glissant sans fin sur d'autres images.

28. G. Deleuze, Pourparlers, Paris, Minuit, I990, p. I09. 


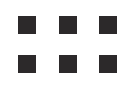

\section{Entre cinéma et photographie: quelques variations de vitesse de l'image contemporaine}

Préambule: photographie, cinéma, et après?

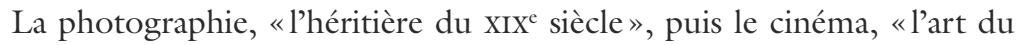
$\mathrm{xX}^{\mathrm{e}}$ siècle », nous ont appris historiquement que les corps enregistrés dans des images étaient des corps identitaires, c'est-à-dire des singularités inscrites dans l'espace et le temps. Je voudrais m'intéresser ici à des états du corps, frappés par les instabilités contemporaines de l'image : des corps vidéo ou numériques - post-photographiques ou post-cinématographiques, mais qui se souviennent, souvent intensément, de leur origine - dont la singularité «improbable» est celle de l'image même; des corps d'image et non des images de corps.

Avant d'aborder quelques-uns de ces improbables corps d'image, je voudrais commencer par cadrer la perspective historico-théorique de mon propos. Alors qu'on a souvent pris l'habitude d'opposer de façon très manichéenne le monde de l'image fixe à celui de l'image mobile, comme s'il s'agissait d'un partage établi et stabilisé, d'une histoire installée - la photographie ici, le cinéma là -, je voudrais en fait remettre en question, ou au moins déplacer, cette dichotomie bloquante, non seulement en la réactualisant par l'examen d'œuvres électroniques contemporaines, mais surtout en la reformulant en termes de régimes de vitesse des images - ce qui revient à introduire la modulation ou la variation continue, l'instabilité comme forme d'être, dans ce qui cesse dès lors d'être une opposition. 
En trente ans en effet, le paysage visuel et théorique a radicalement évolué à cet égard. Dans les années I970-I980, les choses semblaient claires. D'un côté, Roland Barthes dans La Chambre claire ${ }^{\mathrm{I}}$ imposait le concept de «punctum» en jouant la photographie contre le cinéma (avec tous les corollaires sur la pose/pause, le temps mort, l'arrêt sur image, l'effet mortifère de la prise, etc.). D'un autre côté, la philosophie bergsono-deleuzienne du cinéma imposait les concepts d'imagemouvement et d'image-temps, qui reposaient encore entièrement sur l'idée que le film est un défilement régulier d'images reproduisant le mouvement apparent (avec ses corollaires là aussi, le flux, l'emportement, la fuite des images, etc. - et les difficultés, pointées par exemple par Raymond Bellour dans le texte d'ouverture de L'Analyse $d u$ film $^{2}$, si l'arrêt sur image est le geste analytique de base, comment arrêter le fleuve tout en voulant rendre compte de sa puissance-mouvement?). Tout se passait comme si l'un et l'autre, le mobile et l'immobile, le fixe et le mouvant, ne pouvaient exister que dans un rapport d'exclusion réciproque. Il fallait choisir son camp. Cela est assez connu.

C'est ce qui s'est passé après qui m’intéresse. Dans les années I9902000 en effet - c'est seulement aujourd'hui que nous en comprenons toute la portée théorique - il est clair que, sous les effets de la vidéo d'abord et du numérique ensuite, les régimes temporels d'images se sont considérablement élasticisés, rendant de plus en plus obsolètes ou indiscernables les vieux partages. Regardez les films de Wong Kar-wai (la plupart de ses œuvres, de Chungking Express, 1994, ou Fallen Angels, 1995, à 2046, 2004, en passant par In the Mood for Love, 2000), de Gus Van Sant (Gerry, 2002, et la dernière scène tout spécialement), d'Alexandre Sokourov (la formidable et longue séquence d'ouverture de Spiritual Voices, I996) et de tant d'autres. Regardez les vidéoclips ou les publicités pour la télévision (de Michel Gondry en particulier). Regardez les œuvres de cinéastes expérimentaux (de Gianikian et Ricci-Lucchi à Martin Arnold, de Ken Jacobs à Peter Tscherkassky), ou d'innombrables artistes plasticiens (comme Douglas Gordon, qui étire la projection de Psycho, 1994, sur une durée de vingt-quatre heures et celle de The Searchers sur... cinq années, ou encore, exactement à l'inverse, comme Jim Campbell qui, avec ses Illuminated average, 2000, condense par superposition des films entiers - le même Psycho par exemple - en une seule image, ou encore comme

I. R. Barthes, La Chambre claire, Paris, Gallimard, I980.

2. R. Bellour, L'Analyse du film, Paris, Albatros, 1979. 
David Rokeby qui inscrit des mémoires partielles de mouvements dans des images en direct). C'est sans doute une des caractéristiques majeures des modes contemporains de l'image que de changer sans cesse de vitesse, de passer d'un régime à l'autre, et cela en toute souplesse, par variation continue, sans coupure ni changement de nature. Aujourd'hui, le défilement ne s'oppose plus radicalement à l'arrêt, comme s'il s'agissait de deux mondes contradictoires. On n'est plus dans le jeu de «la photographie vs le cinéma». On est au-delà : dans des formes d'images - comment les nommer d'ailleurs? - qui dépassent ce découpage du siècle dernier. Avec la vidéo et le digital, on est passé à l'ère du changement de vitesse permanent. On est, par exemple, dans l'immobile qui bouge (la pose longue, le filé, le tremblé, le panorama en photographie) ou à l'inverse dans le mouvant qui ne bouge, quasi, pas (le plan fixe, le plan long, le bullet-time de Matrix, etc.) et surtout on est dans l'entre-deux, dans les va-et-vient intermédiaires, dans l'espace-temps indécidable des ralentis-accélérés - on ne fait même plus la différence - systématiques. Bien sûr, ce ne sont pas là des formes «nouvelles» - Étienne-Jules Marey, Dziga Vertov, Jean Epstein, avant Jean-Luc Godard, pourraient tracer une sorte de trajectoire archéologique de ce goût exploratoire, scientifique et poétique, des variations de vitesse de l'image. Seulement, elles tendent aujourd'hui, notamment avec les nouveaux supports technologiques, à devenir une norme. Pour nous, professeurs d'analyse de films dans des départements d'études cinématographiques, il suffit de dialoguer avec nos étudiants pour nous apercevoir qu'ils n'ont plus les mêmes rapports perceptifs et même imaginatifs, les mêmes formes d'analyse ou les mêmes modes de penser devant - et avec - les images que la vieille génération de l'«arrêt sur image». Laura Mulvey a même fait de ce constat le point de départ de son dernier livre. ${ }^{3}$ C'est ce changement de fond que je voudrais essayer d'aborder, en prenant appui sur un corpus d'œuvres d'artistes contemporains travaillant cette idée de l'improbabilité spatio-temporelle de la figuration du corps humain.

Les œuvres que je vais aborder dans les pages qui suivent sont au nombre de six. Ce ne sont pas des «films de cinéma» mais des œuvres d'artistes, ou plutôt de plasticiens, usant en toute liberté inventive de la photographie, du cinéma, de la vidéo et du numérique. J'aurais pu bien sûr en choisir beaucoup d'autres, qui travaillent selon d'autres configurations,

3. L. Mulvey, Death $24 x$ a Second. Stillness and the Moving Image, Londres, Reaktion Books, 2005 . 
d'autres modes d'inventions formelles et conceptuelles, mais l'important pour moi, c'est qu'ils s'inscrivent tous dans la perspective globale que je viens d'esquisser. J'ai organisé ce chapitre autour d'un mouvement en trois temps, ou trois vagues successives. La première est une phase pour ainsi dire historique, avec des œuvres du début des années I980, de I98I exactement, c'est-à-dire juste avant la généralisation des variations de vitesses par la vidéo, des œuvres où les modulations de vitesse de l'image sont encore pure affaire de jeu entre photographie et cinéma : il s'agira de Filming Muybridge de Jean-Louis Gonnet, et de 36976 portraits de Hartmut Lerch et Klaus Holtz. Ensuite je présenterai deux œuvres exemplaires des années 1990: Tu de Thierry Kuntzel (I994) et Temps morts d'Emmanuel Carlier (1995). Enfin, je clôturerai avec deux œuvres plus récentes, d'artistes des années 2000: Time machine d'Egbert Mittlestädt (2000) et ... graph d'Emmanuel Carlier (2003).

\section{Filming Muybridge, Jean-Louis Gonnet (198I)}

Voici, strictement, de la photographie qui se fait cinéma. Archéologie imaginaire où le cinéma fait retour sur une phase photographique immédiatement présupposée par ses origines, Filming Muybridge, de JeanLouis Gonnet, n'est pourtant ni un documentaire sur le précinéma, ni un film pédagogique sur l'Américain Eadweard Muybridge ou sur les rapports entre le mouvement et l'immobilité. Ce film est plutôt une invention visuelle qui se donne à voir comme telle, en train de se faire sous nos yeux. C'est une sorte de rêverie zénonienne, donc poétique, sur la scène de l'histoire, de l'image et du mouvement. Trois effets mobilisateurs caractérisent ce travail.

D'abord un effet d'animation, au double sens du mot: s'animer, c'est bien sûr se mettre en mouvement, mais c'est aussi, étymologiquement, générer de l'anima, c'est-à-dire de l'âme, du souffle, de la vie. Comme son titre l'indique, Filming Muybridge se présente à première vue comme la mise en mouvement, l'animation - ou la ré-animation - cinématographique de ces célèbres vues statiques et historiques du photographe américain qui, entre 1872 et I885, sur de grandes planches de I2 ou 24 images successives, décomposaient le mouvement en autant de phases continues, c'est-à-dire d'images fixes qui analysaient la locomotion du corps, humain ou animal - mais Gonnet n'utilise que des figurations du corps humain. Ce qui est fascinant dans ce premier effet, c'est de voir sous nos yeux le mouve- 
ment même qui naît, comme en direct, de ces séries photographiques figées. Cela reste une expérience perceptive et culturelle quasi magique : voir, dans ces images qu'on croyait connaitre par cœur parce que si souvent vues et reproduites, voir par exemple dans le visage de la petite fille du début le sourire qui monte sur sa figure alors qu'elle se tourne vers nous, voir cette naissance du mouvement à partir de l'immobile, comme une montée du plaisir, est quelque chose de littéralement fantastique - ne serait-ce parce qu'on ne voyait pas le sourire comme mouvement dans les photographies. Le sourire n'est plus une position de la bouche, des lèvres, ni même une suite de positions fixées - il n'est d'ailleurs pas visible en tant que tel sur les planches de Muybridge -, mais uniquement du pur mouvement, quelque chose qui ne peut se voir que s'il est en train de se faire, hic et nunc, sous nos yeux, dans le temps de son effectuation. Cela incarne assez strictement la conception, d'ailleurs contemporaine historiquement, de Henri Bergson sur le mouvement: le mouvement ne se décompose pas, il ne peut s'objectiver, ne se mesure pas, ne s'analyse non plus en positions successives; il est une qualité, non une quantité, il ne peut être qu'invention dans un présent sans cesse renouvelé. Chaque seconde du film de Gonnet nous fait éprouver dans notre conscience perceptive cette invention du mouvement en train de se faire et de se défaire, de vivre et de mourir, plutôt qu'en état d'être représenté analytiquement (c'est-à-dire photographiquement).

À ce premier effet, le supplément d'anima par la mise en mouvement, Gonnet en ajoute un second: un effet de mouvement narratifengendré par le travail de montage des images - une naissance de la fiction. En organisant les images dans un certain ordre, Gonnet en vient à ébaucher des moments de micro-fiction virtuelle à partir du matériau photographique de Muybridge. En construisant de véritables petites scènes, ou saynètes, à partir de plusieurs séries distinctes de clichés, par exemple en utilisant ici un homme nu jetant une petite balle, là un autre, dans le même appareil, maniant une batte de base-ball, en montant répétitivement et alternativement les mouvements de l'un puis de l'autre, Gonnet invente un champ-contrechamp, qui n'était pas réellement dans les photographies d'origine, comme si on assistait à une partie de base-ball qui aurait pu avoir été filmée à deux caméras en temps réel. Ailleurs il invente la rencontre d'un jeune homme qui soulève son chapeau avec une jeune fille à l'ombrelle qui marche droit devant elle. Ou encore une femme déshabillée qui se glisse sous les draps d'un lit alors qu'un homme détourne le regard, comme pour ne pas voir cela, etc. Chaque action postule une scène possible dont il suffirait d'articuler les composants juxtaposés à l'origine par Muybridge pour lancer un début 
d'histoire. Ce faisant, Gonnet nous révèle l'extraordinaire part de cinéma virtuel que comportaient de fait les planches de Muybridge, comme si ce dernier avait eu la prescience, involontaire mais éminemment poétique, des effets imaginaires du cinéma avec les corps, les gestes, les trajets, les actions, les enchaînements, les rapports d'angles et les raccords dans le mouvement.

Enfin, une troisième et dernière dimension intervient progressivement au fil du film : elle relève de l'effet de mouvement plastique de la matièreimage utilisée - une naissance de l'abstraction par disparition du motif corporel. En partant des clichés, imprimés, de Muybridge (les planches de l'édition originale de Human Locomotion), en les recadrant / décadrant continuellement, et surtout en les filmant dans des plans toujours plus serrés, isolant des détails de plus en plus petits, Gonnet finit par s'approcher tellement des clichés d'origine que leur figuration s'estompe, qu'on ne peut même plus distinguer les corps ou les actions représentés. Effet de défiguration ouvrant sur l'abstraction visuelle: on ne voit plus que la matière-image elle-même. Les corps et les actes se sont altérés jusqu'à n'être plus que des taches aux limites du reconnaissable, des points ou des trames (la trame d'impression) filmés de tellement près qu'il n'y a plus rien à voir que des agglomérats de zones noires ou blanches, informes, abstraites, à l'image de ce qui se passe dans les agrandissements successifs de la photographie filmée par Michelangelo Antonioni dans Blow-Up (1966). La rêverie zénonienne et nostalgique sur les origines du cinéma - l'effet d'animation qui magnifiait le corps comme apparition du mouvement - et l'effet de fictionnalisation narrative, opérée par le montage de saynètes virtuelles, s'estompent tous deux dans l'informe - tache et trame - de ce qui n'est finalement qu'un anti-photogramme, puisque photographie faite film, disons donc: un cinématogramme.

\section{6 portraits, Karl-Hartmut Lerch et Klaus Holtz (I98I)}

Avec cet autre «film» de I98I, ce sont aussi des corps, plus exactement des visages, des milliers, des dizaines de milliers de visages (36976, dit le titre) qui sont littéralement mobilisés - et en même temps, on va le voir, immobilisés, emprisonnés dans la folie d'une humanité inimaginable mais paradoxalement filmée. C'est le fantasme d'un Corps, totalisant et universel, qui surgit, un Corps-visage absolu, qui serait fait de tous les corpsvisages singuliers du monde. 
36976 portraits est un petit film de quelques minutes - il existe plusieurs versions, longues ou courtes, avec vues de face ou de profil, et aussi des versions installation, pour un, deux, quatre ou six moniteurs - qu'il faut regarder intégralement (il vaut mieux être assis) pour en appréhender la folie figurative. Il ne s'agit rien de moins que de nous donner à voir quelque chose qui a affaire avec l'impensable: le visage de l'humanité, de l'humanité tout entière, ramené ou au contraire emporté par le mouvement des images vers un visage unique, absolu, total, pur, monstrueusement effrayant parce qu'inimaginable - mais rendu illusoirement visible par un dispositif perceptif nouveau: les variations, extrêmes, de vitesse du passage des images.

Les deux réalisateurs du film, Karl-Hartmut Lerch et Klaus Holtz, ont en effet enregistré le portrait filmé (trois photogrammes par personne) d'innombrables individus (36976 donc), tous saisis dans la même posture, le même cadre, sur fond noir. Il y a là des hommes et des femmes, des jeunes et des vieux, des Noirs et des Blancs, des Asiatiques et des Occidentaux, des barbus et des chauves, des ronds et des effilés, des grosses lèvres et des petites bouches, des grands yeux et des gros nez, des sourcils broussailleux et des moustachus aux oreilles décollées... bref, de tout, tous les types physiques humains, mélangés, en désordre, dans une litanie infinie de visages. Les images de film ont été transférées en vidéo pour pouvoir être travaillées par un effet d'accéléré extrême, stroboscopique, pouvant aller jusqu'à près de 20000 images par seconde. Que donne à voir la fusion de ces milliers de types humains anonymes, choisis au hasard, sans souci de psychologie ou de sociologie? Ce désir de voir ce que l'œil humain est incapable de voir par lui-même, de donner figure à un secret éternel, de montrer le vieux rêve métaphysique du cinéma. C'est, malgré tout ce que l'histoire du cinéma a vécu au fil du siècle dernier, faire encore crédit à l'intelligence fondamentale de la machine pour créer cette utopie-cinéma qui prétend filmer l'invisible: le temps pur des corps animé par le vertige des images et condensé dans une figuration impensable. Ainsi Epstein rêvait-il déjà en son temps d'un cinématographe millénaire qui saurait filmer le déplacement des montagnes ou les variations de visage d'une même filiation, de génération en génération. C'est l'idée de donner à voir, par les moyens de la seule variation de vitesse des images filmiques, une image, fantasmatique mais perceptible, d' un visage qui serait la fusion de tous les visages, comme une idée de combinaison alchimique qui aurait trouvé la formule d'un alliage précieux longtemps recherché, la recherche de l'or du visage. Observons le mouvement de ce que nos yeux sont à peine capables de croire. 
Au début, les visages se suivent sagement, chacun apparaissant une brève fraction de seconde, immobile, pour laisser place au suivant. À ce stade, les différences physionomiques sont nettement perceptibles. Le spectateur peut distinguer les visages. Mais très vite le mouvement commençant à s'accentuer, les premiers effets perceptifs se déclenchent. D'abord, notre oil n'arrive plus à séparer les images. Cela va trop vite pour lui et les visages tendent progressivement à se superposer, confondant certains de leurs traits. Puis, la vitesse augmentant, cette superposition s'intensifie et une sorte de fusion s'opère. Non seulement on ne distingue plus les individus, mais une sorte de super-visage tend à s'installer, généré optiquement par l'accumulation des singularités broyées par la haute vitesse. De plus en plus, tous ces visages semblent n'en faire qu'un, générique, lequel tend même, paradoxalement, à se figer de plus en plus. Le centre du (des) visage(s) se stabilise en premier - les yeux surtout sont étonnamment fixes, comme épinglés sur un même centre - pendant que les contours, plus variables, vibrent encore à toute allure. Et lorsque la vitesse atteint son climax, cette variabilité des contours elle-même disparait, se fond dans une moyenne, indistincte et comme unique. On se retrouve alors devant cette chose invraisemblable: un visage parfait, lisse, calme, figé, sans vibration particulière. On le dirait incroyablement stable, solide, immobile, pas vraiment comme du marbre, mais tout de même assez cadavérique. C'est un visage sans différence(s) et sans identité(s). On a presque envie de dire : sans corps propre. Il n'y a plus rien en lui qui distingue homme et femme, jeune et vieux, Occidental et Asiatique,... On a atteint le degré zéro du visage. Cela va tellement vite que rien ne bouge plus - pour nos yeux - et que tous les traits distinctifs, toutes les singularités s'effacent, s'abolissent dans une sorte de schème anonyme et vide. C'est le visage commun de tous les hommes, le visage de synthèse qui a tout absorbé, anéanti, dissous. C'est le visage universel, l'humanité entière visagéifiée dans une apparence unique, produite mécaniquement et perceptivement par un artifice de neutralisation totalitaire des différences. Ensuite, ce sera le processus inverse : le film va décélérer progressivement. Et le visage absolu va devoir se remettre à «relativiser», c'està-dire se remettre à vivre, ses contours vont recommencer à vibrer, des petits signes distinctifs vont apparaitre et croître, réinjectant de la différence, c'est-à-dire du jeu, du mouvement, des écarts, dans un visage qui va finalement cesser d'être unique pour redevenir multiple, divisé, différencié, bref: humain. À la fin du film, on retrouve des individus. On l'a échappé belle; on en tremble encore. On essaie de se convaincre en se disant que 
tout n'était que du cinéma. On doute un peu, mais on respire. Si le film passe en boucle, alors le cauchemar recommence. Sans fin. Soyez-en sûr: à rester devant ce(s) visage(s), vous finirez par y voir le vôtre.

\section{Tu, Thierry Kuntzel (1994)}

Le corps: quoi de plus évident et de plus incertain à la fois, par nature? Imaginez l'installation $T u$ de Thierry Kuntzel. Un monumental cylindre noir, vertical, presque dix mètres de haut, est dressé devant vous, avec une entrée. Vous pénétrez comme dans une cathédrale. Expérience assez monastique de corps dans l'espace. Dans le noir le plus complet de ce volume circulaire, vous découvrez un monde visuel inédit, dans lequel vous vous sentez cerné... par un visage d'enfant. Bien rangées devant vous, dressées en hauteur - il faut lever les yeux - et disposées en cercle tout autour - il vous faudra commencer par votre gauche -, une série de neuf photographies différentes du visage d'un même enfant, en grand format (près de trois mètres de haut pour chaque image), sont alignées. Impression presque de sacré, d'autel, d'élévation du regard, et de l'esprit. Sensation environnementale totale: il faut tourner sur vous-même, comme une toupie, faire le cercle, en observant une à une les photographies, dans l'ordre, comme si vous faisiez de l'image par image. Regardez-les bien, ces grandes images verticales. Elles exposent un visage d'enfant photographié il y a longtemps, dans le style «soigné»-par l'éclairage, par l'habillement, par les poses qui lui sont demandées. Il a un regard fascinant et des postures exemplaires. Il ne regarde pas l'objectif mais détourne plus ou moins les yeux puis la tête, vers la droite, vers le haut, etc., selon un écartement qui se marque de plus en plus au fur et à mesure de l'avancée du cercle. Ce côté détaché, ou fuyant, ou décalé, lui donne une attitude un peu curieuse, qui interpelle doucement, qui peut prêter à sourire. On se demande quel photographe - de quartier, de village? - a pu faire de telles prises de vue. Qu'est-ce qu'il disait à cet enfant pour lui faire tourner la tête ainsi, toutes les photographies ayant visiblement été prises lors d'une seule et même séance? Et bien sûr, on se demande qui est cet enfant: Kuntzel vraisemblablement. De quand cela date: l'après-guerre sans doute. On pense à des photographies tirées d'un album de famille. Et l'on fait défiler les pages de l'album, la galerie de portraits, comme on ferait défiler un film, ou au moins un flip book. La une, la deux, la trois, etc. On compare, on avance, on tourne. Ces images, vous ne pouvez 
jamais les voir toutes ensemble puisqu'elles forment un cercle qui vous entoure. À cause des directions éclatées du regard de l'enfant et des poses très variées de ce qui est tout de même un corps unique, la façon dont les images se différencient les unes des autres est particulièrement fascinante. On tourne sur soi-même pour faire avancer sa vision, comme on tourne dans un panorama pour tout voir, et à chaque étape, on compare avec les autres postures, les autres mimiques. Lorsqu'on arrive à la fin, à la dernière image, qu'on tombe en arrêt, et qu'on s'interroge. La neuvième image nous accroche par sa façon d'être, plus intrigante encore, gênante même. On se pose des questions: est-ce bien une image dans la suite des autres? On a l'impression que quelque chose la distingue. Peut-être la lumière, sa texture, sa place en fin de course, son sujet? Et puis on comprend: elle bouge. Le visage s'y donne en mouvement. Un mouvement ralenti et irrégulier, mais un mouvement réel, bien visible finalement si on reste un peu. C'est le même visage d'enfant, le même type de portrait photographique à l'ancienne. Mais c'est animé. Le visage y exprime des mimiques variées. Il change de position. Et même, plus fascinant encore, il passe par des postures tellement inattendues qu'on a du mal à les considérer comme vraisemblables: comme s'il y avait là des expressions impossibles, des mimiques impensables, des positions d'éléments du visage et du buste qui ne peuvent être réelles - le mouvement des épaules en particulier est tout à fait incroyable. C'est inquiétant, et en même temps, pas tout à fait, pas complètement: il y a aussi quelque chose de familier dans ces postures, tellement familier qu'on y reconnait des expressions déjà vues, des positions qui figuraient dans les images précédentes. Tout s'éclaire alors: la dernière image du dispositif circulaire n'est pas une photographie mais une image vidéo, projetée exactement au format et dans les tons des photographies précédentes (recadrage vertical, filtrage sépia), une image vidéo animée donc, qui montre, en mouvement continu, une sorte de visage de synthèse, comme pouvait l'être celui de 36976 portraits à sa vitesse maximale, mais ici obtenu à partir des images antérieures par le procédé technique du morphing. Les mouvements du visage de l'enfant - singuliers, troublants, bizarres - ont donc été fabriqués numériquement par la machine à partir de photographies déjà là. C'est pourquoi ils sont irréguliers, un peu saccadés, souvent comme au ralenti. C'est pour cela, surtout, qu'ils sont parfois improbables, sinon invraisemblables. L'ordinateur a calculé toutes les formes théoriques intermédiaires entre les états du visage donnés par les photographies, qu'elles puissent exister ou non. Il a inventé les étapes de transition, comblé les manques, rac- 
cordé les phases figées par la photographie, fluidifié les enchaînements, liant les images dans une animation artificielle, mathématique, qui est venue donner vie par le mouvement à ce fantôme fabuleux. Tout photographique qu'il paraisse, ce mouvement numérique du corps de Kuntzel enfant ne correspond donc à rien, à aucun mouvement réel; c'est une fiction, une aberration, une vue de l'esprit. Il n'a ni vitesse, ni rythme, ni forme, ni ressemblance avec un déplacement effectif du corps. Seuls les points photographiques, qui ont servi de matrice pour calculer l'invention des mouvements intermédiaires, avaient encore un pied dans le réel. Le reste est un rêve de machine. Mais un rêve visible, perceptible, inscrit dans et surtout par l'image. Ce n'est plus l'image d'un corps mais un corps d'image. Dans cette œuvre majestueuse Kuntzel s'est fabriqué une fantastique machine à autoportrait improbable. L'effet est absolu et les abîmes qu'il ouvre, insondables.

\section{Temps morts, Emmanuel Carlier (I995)}

Tout le travail d'Emmanuel Carlier inscrit sous le titre Temps morts s'élabore à partir d'un dispositif de base, à la fois simple dans ses principes et complexe, voire souvent spectaculaire, dans ses effets. Ce dispositif ouvre à un véritable vertige de la pensée et de la sensation en nous confrontant à ce paradoxe: comment se déplacer cinématographiquement au cœur même d'un instant photographique? Comment bouger dansl'immobile?

Il faut bien comprendre le procédé de fabrication: d'abord il y a une scène, entièrement organisée pour l'image, avec acteurs, éclairage, accessoires, etc. C'est un espace souvent circulaire, cerné, autour duquel l'artiste peut tourner pour observer «le spectacle» sous toutes ses coutures, un peu comme une piste de cirque ou de music-hall. À l'intérieur de cette zone de jeu, «l'action» se prépare, puis se déroule: des corps, nus le plus souvent, d'hommes et de femmes, sont disposés dans des postures précises. Les gestes et positions impliquent toujours des mouvements, souvent extrêmes. Ils sont liés pour la plupart à des jeux et des jets d'eau sur les corps: écoulement, éclatement, jaillissement, chute, explosion. Une grande intensité anime ces actions corporelles, au bord d'une certaine violence symbolique. Ce sont des acteurs de la troupe de théâtre catalane Furia del Baus qui jouent le jeu.

Ensuite il y a le dispositif technique de prise de vues: tout autour de cet espace scénique circulaire, une batterie d'appareils photographiques, 
plusieurs centaines, tous identiques, tous tournés vers le centre de la scène, rigoureusement disposés en cercle à distance égale, et strictement synchronisés sur la même fraction de seconde par un flash électronique. Ils vont photographier la scène exactement dans le même instant de temps et sous autant d'angles légèrement décalés. L'action se prépare. Tension extrême, comme sur un tournage de cinéma. Noir. Cri. Explosion-jaillissement. Des corps, de l'eau, de la lumière. Flash. Captation magnifique d'un éclat de l'action, figé à son acmé, qui est à la fois unique temporellement et démultiplié spatialement. Au terme de la prise de vues, Carlier dispose donc d'une série de plusieurs centaines de photographies de la même action, toutes strictement synchrones, d'une netteté absolue - elles ont été prises au millième de seconde, et c'est ce même point de temps qui a été saisi, figé net dans chaque image -, et captées à partir de points de vue légèrement décalés mais en progression régulière au fil du cercle d'appareils qui entouraient la scène.

Il ne reste alors plus qu'à organiser le montage-animation de cette série d'instantanés, comme si chaque image devenait le photogramme d'un film à mettre en mouvement. Un double effet se met ainsi en place à partir de cette série de photographies: d'un côté un effet comparable à celui de Muybridge, de décomposition en vues successives, et d'autre part un effet de vision panoramique circulaire - on a tourné tout autour de la scène en accomplissant une boucle complète. Mais, à l'extrême différence des vieilles images chronophotographiques, il s'agit moins ici de décomposer temporellement un mouvement que de dé/re-composer spatialement un non-mouvement, un instant figé net, le même sur tous les clichés, mais vu différemment, selon une rotation progressive du point de vue. C'est cela qui est proprement vertigineux dans le travail de Carlier : le temps a été arrêté, le mouvement du geste ou de l'acte a été figé, l'eau, qui giclait, éclaboussait, tombait, est littéralement suspendue, presque solidifiée, une fois pour toutes, dans toutes les images, exactement au même point de temps. Et c'est ce point temporel, cette coupe instantanée qui est explorée progressivement, point de vue par point de vue, dans l'ordre des images, restituant ainsi un parcours du regard qui s'anime (anima), au sens cinématographique, sur le mode d'une sorte de panoramique, fictif, tournant tout autour de la scène, mais une scène préalablement immobilisée par la photographie. Comme dans un panorama inversé, nous déroulons notre regard dans l'espace-temps fait image d'un instant de temps immobilisé. Voilà l'extraordinaire paradoxe de l'expérience des Temps morts de Carlier: le spectateur se déplace à l'intérieur, et pour ainsi 
dire dans l'épaisseur, d'une image fixe, il explore un mouvement perceptif circulaire qui prend du temps pour s'accomplir et qui se déroule dans un espace virtuellement en trois dimensions, une action qui a été absolument figée dans une même infime fraction de seconde. C'est le contrepoint exact des poses longues, des vibrations du bougé dans l'image fixe et de ses effets picturaux de surface, puisqu'il s'agit ici d'un mouvement effectif dans ce qui semble une image nette, quasi en relief, d'un même geste immobilisé à l'acmé de son mouvement. Sensation étonnante d'une véritable sculpture ciné-photographique de l'instant, où l'on a l'impression de tourner autour d'une tranche de temps.

Notre sensation visuelle est bien celle d'un paradoxe sur lequel nous butons en nous perdant en conjectures: comment se déplacer, cinématographiquement, dans un instant de temps figé, photographiquement? Nous sommes bien au cœur de la photographie, de sa mythique capacité à fixer le mouvement dans une fraction de seconde médusante. Et pourtant, nous devons bien admettre cette évidence : l'image que nous voyons est en mouvement: le spectateur voit se dérouler devant lui ce qu'il doit bien considérer comme un travelling circulaire tournant autour de ces corps, pourtant figés et suspendus dans l'immobilité totale. C'est cela qu'il ne comprend pas. Comment être à la fois dans un mouvement de caméra cinématographique et dans un instantané photographique? Comment peut-on à la fois arrêter le temps (le couper, le figer net) et explorer l'espace de cet instant dans la durée (le déroulement) d'un mouvement panoramique total, car on tourne plusieurs fois complètement autour de ces corps suspendus? Comment peut-on se déplacer circulairement - ce qui prend du temps - dans ce qui n'est pourtant qu'un instant infinitésimal? Comment peut-on être à la fois dans le mouvement et dans l'immobile? Comment peut-on tourner autour d'une tranche de temps? Sans réponse à ces questions, si ce n'est que ce paradoxe a lieu sous ses yeux, le spectateur, comme devant, ou dans, l'œuvre circulaire de Kuntzel, ne peut que s'enfoncer dans l'abîme perceptif et spéculatif du dispositif machiné par le diabolique Carlier.

$T u$ de Kuntzel et Temps morts de Carlier sont deux installationsdispositifs qui, au milieu des années 1990, ébranlent sérieusement notre conscience, perceptive autant que métaphysique, car l'objet sur lequel portent les inventions de mouvements, le corps, est un objet fantas(ma)tique qui engage profondément notre identité, notre rapport à nous-même comme image. Les machines à mouvements impossibles mises en branle ici (le morphing, le temps mort) sont d'autant plus inquiétantes qu'elles 
affectent non seulement notre activité perceptive (le regard, qui doute de ce qu'il voit) mais aussi notre activité cognitive (notre pensée de la conscience de soi comme corps). Qu'est-ce que voir? Qu'est-ce qu'une image? Qu'est-ce qu'un corps? Et finalement, qu'est-ce que nous sommes, comme objet et comme sujet, optique et éthique, physique et psychique? Voir est un mouvement. Une image est un mouvement. Un corps est un mouvement. La perception, la conscience et la mémoire sont des mouvements. Et tous ces mouvements n'en font pas un, qui les synthétiserait dans un grand tout homogène (pas de convergence vers l'unité) : ils exercent chacun leur force singulière et donc interfèrent entre eux sur un mode ouvert et incertain, se combinent, se répondent, se relancent, se contredisent, se dédoublent. Cette improbable symphonie de mouvements nous transporte dans un tourbillon, qui est au moins autant philosophique que spatial, autant de pensée que de regard.

\section{Time Machine, Egbert Mittlestadt (2000)}

L'installation d'Egbert Mittlestädt Time machine se présente sous la forme d'un cyclo (un vaste cylindre) translucide, sur lequel est imprimée une grande photographie circulaire (formant une image panoramique à 360 degrés). Celle-ci représente un carrefour dans une ville allemande, photographié (à l'aide du dispositif de prise de vue connu sous le nom de Roundshot) depuis le centre du carrefour et faisant le tour complet du champ de vision à partir de ce point de vue. Le cyclo est suspendu au plafond à hauteur de visage. Par transparence, on peut voir cette image circulaire aussi bien de l'extérieur que de l'intérieur, car le cercle est assez large (trois mètres et demi de diamètre) pour contenir en son sein plusieurs spectateurs, qui y prennent place comme on s'installe dans la rotonde d'un bon vieux panorama, ou dans une fusée avant de faire un voyage dans l'espace-temps. Vous êtes prêt pour l'intersidéral? La photographie imprimée offre le spectacle figé, pour l'éternité, d'une animation urbaine diversifiée (rues, piétons, cyclistes, voitures, tout ce qui circule), restituée dans un effet de continuité visuelle ininterrompue, comme dans un panoptique qui aurait tout immobilisé dans une seule prise de vue étalée tout autour de soi. Pour observer complètement cette image fixe, invisible comme totalité en un seul coup d'œil, le spectateur doit évidemment tourner sur lui-même et faire pivoter son regard. Accomplir le tour complet lui prend un certain temps, ou plutôt un temps certain - même 
si, quand il revient à son point de départ, il se retrouve au même point de temps qu'au début. Petit paradoxe de la photographie panoramique: du temps a passé - celui du mouvement du regard -, mais grâce au figement photographique, rien n'a bougé, et le temps - représenté - est resté le même. Temps vivant - le regard - et temps mort - l'image - se retrouvent, et tout peut recommencer indéfiniment.

Là où l'installation de Mittlestädt prend toute sa dimension, sa pleine puissance de voyage dans l'espace-temps, au point de paraitre conceptuellement inconcevable, c'est par la présence d'une deuxième image, en mouvement celle-là, qui vient se superposer, par projection, sur la première. Mittlestädt avait en effet couplé à son appareil photographique Roundshot une petite camera vidéo numérique orientée exactement dans la même direction et donc filmant exactement la même chose - mais en mouvement. Dans l'installation, cette image vidéo est donc projetée sur l'image photographique. Au centre du cylindre, faisant axe et incarnant le point de vue, une colonne munie d'un plateau tournant fait pivoter le projecteur vidéo qui envoie son image sur l'écran qu'est le cyclo. Le spectateur voit donc désormais sur l'image fixe du panorama photographique une autre image, qui, elle, est doublement en mouvement puisque, d'une part, c'est une image-mouvement en elle-même (un panoramique vidéo) et que, d'autre part, elle est projetée par un projecteur lui-même en mouvement, rotatif, au sommet de sa colonne centrale. Le résultat le plus frappant de ce dispositif sophistiqué est l'extraordinaire corrélation qui s'établit entre l'image vidéoprojetée, avec son double mouvement, en soi et hors de soi, et l'image figée de la photographie qui fait écran - un écran spatialisé où le temps du parcours est annulé par le temps de la représentation. Tout tient au fait que l'image vidéo projetée sur le cyclo donne à voir la même chose que l'image photographique - mais en mouvement. Il faut le voir pour le croire, et non y croire pour le voir. L'image vidéo montre des choses en mouvement: par exemple, un cycliste qui avance, une voiture qui passe, un piéton qui marche. Et elle projette cette chose en mouvement sur la partie de la photographie qui a enregistré exactement la même chose en le figeant, le même cycliste ou le même piéton. La superposition est assez judicieusement ajustée. Elle n'est pas, ne peut pas être strictement exacte évidemment, mais elle est efficace, et d'autant plus troublante qu'elle s'effectue dans la durée, tout au long du déplacement rotatif complet de la projection (c'est une question de synchronisation) : à mesure que l'écran de la projection vidéo avance sur l'écran photographique qui lui sert de fond, cet ajustement du mouvement sur 
l'immobile (de la vidéo sur la photographie) ne cesse de se faire, de se défaire, de se refaire, infiniment, multipliant à perpétuité la fascinante vision de l'improbable conjonction. Plus on s'absorbe dans cet accord visuel de mouvements incompatibles, plus on en découvre des enjeux. Ainsi, on comprend vite que l'on doit regarder à la fois le rectangle vidéo qui progresse - il est cernable en un coup d'œil lui, et sa luminosité plus forte attire notre regard - mais aussi ce qui, dans la photographie, l'entoure immédiatement, avant et après, et change sans cesse: on observe alors que ce qui se trouve dans la photographie juste avant que l'image vidéo vienne le rejoindre, c'est, dans le fond, le futur de ce mouvement - de fait, on jette fréquemment des coups d'œil dans cette direction pour prévoir la mise en mouvement, pour repérer telle personne, encore immobile, dont on se met à attendre impatiemment la mise en route dans et par l'image vidéo qui la rattrape. Et pareillement, on comprend que ce qui figure dans la photographie juste après le passage du cadre vidéo, c'est justement le passé de ce mouvement qui s'en va, est déjà ailleurs, a disparu en nous laissant sa mémoire immobilisée. En d'autres termes, dans cette perspective, seule l'image vidéo est vécue comme un présent - parce qu'elle est mouvement - alors que la photographie nous en livre une anticipation ou une postérité figées. Le mouvement est un présent parce qu'il est en cours. Passé et futur sont immobiles parce qu'ils sont inscrits à demeure à leur place. Mais le mouvement, parce qu'il est toujours en cours et qu'il est rotatif à 360 degrés, revient sur ce qu'il a laissé, refait de son passé un nouveau futur, repasse et repasse, dans un trajet d'éternel retour, réactualisant perpétuellement chaque portion de l'image fixe. Ce qui cesse de faire un présent séparé, un futur ou un passé figés. Tel est le paradoxe induit par le principe de circularité de l'ensemble: littéralement, ce circuit n'a pas de fin, ni de début. L'espace est circulaire et le temps cyclique. Le mouvement coïncide avec l'immobile; le présent avec le passé et le futur; l'instant avec la durée; le temps avec l'espace; le regard qui progresse avec le temps qui reste; l'image qui demeure avec le temps qui passe. Cela tourne, cela tourne jusqu'au vertige.

\section{...graph, Emmanuel Carlier (2003)}

Quand le spectateur découvre ...graph, il est d'abord confronté à une imposante machinerie, exposée pour elle-même, nullement dissimulée: une architecture de tubes de métal posée au sol, deux longs rails, un sol 
fait de billes d'argile répandues, un chariot mobile, un moteur électrique, un tableau de commande, un grand écran plasma horizontal qui va et vient en faisant beaucoup de bruit : voilà de la matière lourde, du mouvement, des sons; c'est assez impressionnant, inquiétant même à certains égards. Entre ingénierie et bricolage, Carlier met en espace non seulement une image, le résultat d'un processus, mais aussi un engin, qu'il a fabriqué luimême. On pense assez vite à d'autres machineries techniques imposantes, et non dépourvues d'esthétique, dont la pièce de Carlier parait ici une métaphore possible: métaphore industrielle des machines outils dans une chaîne de montage à l'usine; métaphore cinématographique du dispositif de tournage d'un travelling, avec les rails et le chariot; métaphore informatique de l'imprimante, qui inscrit une image à partir des mouvements horizontaux d'allers et retours de sa cassette, etc. Mais en fait, c'est la métaphore médicale qui domine: comment ne pas voir en effet dans cette machine à mobiliser l'écran une sorte de scanner, qui balaie et découpe à la fois son objet, d'autant que le motif convoqué à l'image - dans l'écran plasma - est le corps humain. ... graph fonctionne littéralement comme un scanner, avec son écran magique qui va et vient, qui «découpe» un corps d'homme couché, zone par zone, en le balayant de la tête aux pieds et des pieds à la tête, et en nous le donnant à voir serré de près, scruté dans le moindre de ses détails anatomiques. Regard clinique. Ce corps plasma (le mot lui-même est intéressant...), étendu sur les mêmes billes d'argile sombres que celles qui sont dans la salle d'exposition sous la machinerie, se donne à nous entièrement nu, comme sur une table d'examen.

L'impression est étrange : il n'y a évidemment aucun corps réel visible sous l'écran, mais on pourrait dire qu'il y en a un de virtuel, intensément présent. Le fait de voir dans l'image ne serait-ce que des fragments de ce corps, immobile dans un premier temps, agité de soubresauts violents dans un second temps, puis de voir, par les mouvements de va-et-vient répétés de l'écran, ces fragments de corps reconstituer fictivement un ensemble articulé complet - un peu comme les décompositions du mouvement dans les photographies de Muybridge permettaient d'imaginer tout un geste ou tout le trajet accompli par un corps -, donne avec force le sentiment de présence d'un corps virtuel. Nous ne voyons effectivement qu'une image de corps morcelé, mais l'exhibition frontale (en particulier lorsque l'écran donne à voir, crûment, froidement, le sexe masculin de l'acteur), les mouvements de passage répétitif de l'écran, et, surtout, l'horizontalité du trajet de l'image (il est rare de voir une image couchée, nous sommes 
tellement habitués à les voir debout, dressées, verticales, que nous en venons presque à associer une idée de mort à cette horizontalité): tout cela invente un curieux effet de présence invisible du corps sous l'écran créant la figure du scannage. Et ce, d'autant plus fortement que le spectateur comprend immédiatement que l'écran plasma qui passe et repasse à vitesse régulière au-dessus d'un corps absent, mais rêvé, reproduit en fait très exactement les mouvements de travelling de la caméra qui a filmé ce corps, alors bel et bien présent physiquement, avec le même dispositif des rails, du moteur, du sol, etc. C'est en se substituant à la caméra et en reproduisant à l'identique ses mouvements que l'écran-scanner invente un corps virtuel, qui, pour n'être pas visible comme tel, n'en est pas moins aussi fantasmatique que le corps impossible inventé par le morphing de Kuntzel à partir de ses photographies d'enfance. Nul doute que ce corps d'homme hantera longtemps notre conscience et notre mémoire. 
Récits 



\section{Du visage au dos: en relisant Levinas avec les frères Dardenne}

Avoir honte de son immoralité : c'est un degré sur l'échelle, au bout de laquelle on a honte aussi de sa moralité.

Nietzsche, Par-delà le bien et le mal.

Avant d'évaluer la fertilité d'une confrontation du cinéma des frères Dardenne à la pensée d'Emmanuel Levinas' ${ }^{1}$, il faut noter que ces derniers lient eux-mêmes leur œuvre à celle du philosophe français, et ce dès sa conception. ${ }^{2}$ Le journal de Luc Dardenne, $A u$ dos de nos images ${ }^{3}$, récit de leur travail de I99I à 2005 , est en effet parsemé de brèves mais érudites références au corpus levinassien. Si ce dernier y est cité entre autres références, parmi les écrits de romanciers, poètes, philosophes et autres cinéastes, il signale néanmoins une source d'inspiration et d'aspiration éthique pour les deux réalisateurs belges, lesquels cherchent en permanence à déstabiliser les idées reçues sur le cinéma. En cela, leur approche est explicitement, sinon exclusivement, liée aux fondements éthiques de la pensée levinassienne. La reformulation de la question éthique qu'opère cette dernière

I. Une version de cet article a été publiée en anglais: S. Cooper, «Mortal ethics. Reading Levinas with the Dardenne Brothers ", Film-Philosophy, vol. II, nº 2, août 2007, p. 66-87, [en ligne], [URL: http://www.film-philosophy.com/2007vinn2/cooper.pdf], consulté le 24 février 20IO. Je remercie J. Game très chaleureusement d'avoir traduit ce texte de l'anglais.

2. Pour une analyse brève mais suggestive du rapport entre le philosophe et les cinéastes, voir J. Mai, "Corps-caméra. The evocation of touch in the Dardenne La Promesse (1996)», Contact! The Art of Touch, M. Crowley éd., vol. 47, n 3 de L'Esprit créateur, 2007, p. I33I44; et M. O'Shaughnessy, "Ethics in the ruin of politics. The Dardenne Brothers », Five Directors: Auteurship from Assayas to Ozon, K. Ince éd., Manchester, Manchester University Press, 2008, p.59-83.

3. L. Dardenne, $A u$ dos de nos images, Paris, Seuil, 2005. 
crée une fissure à la racine de l'ontologie. En écho, les films des Dardenne lancent comme un défi d'inspiration levinassienne à l'être du cinéma. Dans ses moments extrêmes, tuer ou ne pas tuer est la question centrale que pose leur cinéma. Cette reformulation implicite de la question ouvrant le fameux monologue de Hamlet amène une réflexion sur l'être et le nonêtre pour l'orienter vers la question de la survie de l'autre. Le passage de l'être au non-être, impliquant la mort du sujet, est ici déplacé au profit d'une préoccupation quant à l'acte de tuer quelqu'un - ou de ne point y parvenir. Ce glissement de la problématique du mourir à celle du tuer est au cour de l'éthique cinématographique des Dardenne.

Dans l'épigraphe à ce chapitre, Nietzsche nous fait monter ou descendre l'échelle de la honte, de l'immoralité jusqu'au questionnement de toute moralité. De fait, la question éthique elle-même trouve son origine dans une semblable interrogation de la moralité. Faisant parcourir à l'apophtegme de Nietzsche un pas orthographique supplémentaire, je voudrais interroger ici ce que signifie - et implique - de parler de mortalité et d'immortalité plutôt que de moralité et d'immoralité sur un terrain éthique clairement post-nietzschéen. Dans leurs films, les Dardenne traitent de l'immortalité afin de traiter la question de la mortalité de manière critique. Ils font référence à l'opinion commune selon laquelle l'art offre aux sujets humains la chance de devenir immortels, c'est-à-dire l'impossibilité du mourir. Mais plutôt que d'abonder dans ce sens, ils se demandent si l'art - y compris leurs films - peut instituer l'impossibilité de tuer l'autre, moment crucial de l'éthique levinassienne. La célèbre association godardienne entre travelling et moralité suggère comment une telle question éthique peut être posée à travers l'étude de la forme du film, de sa matière, aussi bien qu'en termes de contenus thématiques. Qui plus est, les Dardenne développent leurs observations aux fins d'expliciter la rencontre entre le spectateur et leur ouvre. Ils ouvrent ainsi à l'altérité les différents sujets de leurs films: le sujet-film et le sujet-spectateur.

Les Dardenne ont débuté par le documentaire dans les années 1970. Leurs premiers films étaient à dominante sociale et ne rencontrèrent ni succès commercial ni attention critique. ${ }^{4}$ Ce n'est qu'avec La Promesse, en 1996, qu'ils imposent leur style et que leurs films sont immédiatement reconnus et salués par la critique. Depuis cette date, trois autres fictions ont été écrites, produites et réalisées par les frères, chacune à petit budget:

4. Pour une analyse approfondie des premiers films, voir M. O'Shaughnessy, «Ethics in the ruin of politics», art. cité. 


\section{Du visage au dos}

Rosetta (1999), Le Fils (2002) et L'Enfant(2005).5 Les traits distinctifs de ces films sont la présence d'acteurs non professionnels aux côtés de comédiens confirmés, une façon de filmer caméra à l'épaule, de privilégier la prise de son directe et de ne jamais recouvrir l'image d'une musique non diégétique. Situées principalement aux alentours de Seraing, région industrielle en déclin à la périphérie de Liège, ces fictions explorent sans indulgence les dures réalités de l'immigration, du chômage et de la marginalité dans la société belge. Les décors de la Meuse, des bois, des routes, les no man's land des alentours confêrent une matérialité brute aux situations sociohistoriques des personnages, ce qui sert l'objectif revendiqué des cinéastes d'observer l'humanité contemporaine - non pas d'une façon générale ou abstraite mais dans des situations concrètes extrêmes. Les frères Dardenne parlent souvent du cinéma comme d'un moyen d'accéder à l'humanité, et sa vocation est selon eux de capturer le regard humain, qu'ils conçoivent néanmoins comme étant à double tranchant, en ce qu'il exprime et prohibe simultanément la pulsion meurtrière. À cet égard, les deux réalisateurs marquent immédiatement une dette explicite envers Levinas en ce que, dans sa conception du visage, le doublet désir et interdiction de tuer est fondamental. Cependant, l'éthique levinassienne entretient une relation instable au monde phénoménologique. Et cette instabilité constitue paradoxalement un lien entre monde matériel et sphère de l'immatériel que les Dardenne s'efforcent de rendre en termes cinématographiques. ${ }^{6}$ Ce vaet-vient est riche d'enseignements sur les façons dont la relation entre film et subjectivité, reliée au doublet corps et esprit, a été théorisée jusqu'à présent.

\section{Rencontres face à face}

Levinas développe son système éthique dans un dialogue poussé avec la phénoménologie occidentale, principalement dans deux livres majeurs: Totalité et infini: essai sur l'extériorité (I96I) et Autrement qu'être ou audelà de l'essence (1974).7 Selon lui, la philosophie occidentale a le plus

5. Cet article a été écrit avant la sortie de leur film le plus récent, Le Silence de Lorna, en 2008.

6. Pour d'autres analyses de cette relation entre le cinéma et la philosophie de Levinas, voir S. Cooper, Selfless Cinema? Ethics and French Documentary, Oxford, Legenda, 2006; et The Occluded Relation: Levinas and Cinema, S. Cooper éd., vol. II, nº 2 de Film-Philosophy, août 2007, [en ligne], [URL: http://www.film-philosophy.com/index.php/f-p/ issue/view/I3], consulté le 24 février 2010.

7. E. Levinas, Totalité et infini: essai sur l'extériorité, La Haye, Martinus Nijhoff, 197 I [196I]; E. Levinas, Autrement qu'être ou au-delà de l'essence, La Haye, Martinus Nijhoff, 1974. 
souvent été une ontologie : l'altérité y a systématiquement été réduite à la mêmeté - réduction dans laquelle rien d'autre que de l'être ne peut apparaître. Bien que les entreprises phénoménologiques de Husserl et de Heidegger demeurent des références centrales de son œuvre, Levinas cherche en fait à aménager un espace de transcendance interne à la dimension de l'apparence et de la luminosité si cruciale pour la phénoménologie. Les principaux concepts qu'il développe dans Totalité et infini pour déterminer la relation éthique sont le visage et la caresse, lesquels sont théorisés comme donnant lieu à une relation d'altérité jamais entièrement assimilable par les sens, et surtout pas par la vue et le toucher. Ces connexions sensibles sont selon Levinas des gestes potentiellement totalisants, qui en cela réduisent l'altérité à la seule expérience du sujet, dès lors l'autre à du même plutôt que de créer pour lui des possibilités d'émerger par et dans ses propres termes. C'est le langage qui, pour Levinas, permet de transcender de tels gestes totalisants. Le visage est avant tout un visage qui parle, qui prononce les mots du commandement: «Tu ne tueras point.»

Ce concept de visagéité est au cœur des quatre films des frères Dardenne réalisés entre 1996 et 2005 , comme l'est l'écho de son commandement. Ces films constitueront mon corpus. Comme l'a noté Martin O'Shaughnessy, ils tournent tous, de près ou de loin, autour de la question du meurtre ainsi que d'autres actes d'une extrême violence symbolique, comme le fait de supprimer l'existence sociale de quelqu'un. Ils traitent aussi de la façon dont des actes meurtriers peuvent être répétés. Et cependant, chacun brise le cycle d'une telle répétition, menant le spectateur du meurtre à sa prohibition. Leurs épilogues rédempteurs mais laïques font briller une lueur d'espoir dans un univers glauque, et suggèrent que le futur peut être différent du passé ou du présent. Dans la position levinassienne des deux réalisateurs, tout l'enjeu est de faire parler le monde via le cinéma, lui faire dire ce qui est essentiel à la relation éthique entre êtres humains, mais qui ne peut précisément pas être saisi par la vue. En d'autres termes, il s'agira de s'interroger sur la façon dont le cinéma des frères Dardenne, dans ses façons de filmer le corps, parvient à donner forme à l'optique spirituelle de l'éthique de Levinas. C'est avec ce programme à l'esprit que je considérerai d'abord deux films qui traitent de meurtre au sens propre: La Promesse et Le Fils.

La Promesse s'attache essentiellement aux vies d'Igor (Jérémie Renier), Roger (Olivier Gourmet) et Assita (Assita Ouedraogo). Cette dernière est mariée à Hamidou, un travailleur immigré du Burkina Faso illégalement employé par Roger, un trafiquant de main-d'œuvre. Hamidou tombe d'un 
échafaudage accidentellement et Igor, le fils de Roger, lui sauve la vie en bloquant l'hémorragie consécutive à la chute. Tandis qu'Igor lui porte secours, Hamidou lui fait promettre de s'occuper d'Assita et de leur tout jeune enfant si quelque chose devait lui arriver. Lorsque Roger découvre le corps blessé d'Igor, il retire la ceinture faisant office de garrot et le recouvre entièrement, entraînant ainsi sa mort. La promesse d'Igor permet alors de tester sa loyauté envers son père puisque ce dernier cache le décès aux autorités. Les interrogations d'Assita quant au lieu où se trouve son mari menacent Roger et la mettent en danger elle-même, car il ne reculera devant aucun moyen pour préserver sa situation.

Les frères Dardenne expliquent que le film est bien de son temps en ce qu'il explore le thème du trafic d'êtres humains et son impact sur la démographie belge. ${ }^{8}$ Un critique français déclare que cet aspect du film éclaire la réalité des sans-papiers en France et le mouvement pour la révision des lois Pasqua. ${ }^{9}$ Les réalisateurs insistent cependant sur l'histoire d'un fils pris entre son père et la vérité. ${ }^{10}$ Roger, le père, exerce une forte présence dans la vie de son fils Igor, même lorsqu'il est hors champ (le klaxon de sa fourgonnette, par exemple, ou sa voix envahissent l'espace de son fils). La présence paternelle est aussi figurée par un cadeau lorsqu'au début du film il offre une bague à son fils. Dans plusieurs scènes, cet anneau joue le rôle, visuel comme matériel, d'une métonymie rappelant la présence du père, sa persistance. Igor finit par le retirer et le vendre au profit d'Assita. Cette coupure symbolique présage la rupture réelle à venir. Dans sa proximité grandissante avec Assita, Igor change peu à peu, ce qui l'éloigne de son père au fur et à mesure que le film progresse.

Dans les scènes qui les réunissent lui et elle, le regard de la jeune femme est souvent le plus direct et soutenu, quand celui d'Igor se détourne vers le sol ou se perd au loin. Luc Dardenne soutient que cela est dû à l'incapacité d'Igor de répondre à l'impératif moral qu'il perçoit dans ce regard qui le dévisage. ${ }^{\mathrm{II}}$ Igor est fasciné par la foi d'Assita et l'interroge souvent à ce sujet. L'islam est suggéré lorsqu'elle achète un mouton au marché pour célébrer la fin du ramadan. Tout au long du film, Assita apparait comme

8. Cité dans A. Campion, "La promesse des clandestins», Le Journal du dimanche, I3 octobre 1996. Toutes les citations d'articles de journaux viennent de la base de données de la Bibliothèque du film à Paris.

9. G. Lefort, "Belle tenue pour une Promesse», Libération, I6 octobre 1996.

IO. Cité dans A. Campion, "La promesse des clandestins», art. cité.

I I. L. Dardenne, $A u$ dos des nuages, ouvr. cité, p. 57. Désormais, les numéros de page donnés dans le texte sans indication supplémentaire renvoient à cette édition. 
une autorité qui provoque et corrige Igor. Leur relation est physiquement distante mais un échange de mots ou de regards suffit à la caractériser. Dans une scène à la fin du film Igor pleure et déclare à Assita regretter de l'avoir aidée. Puis il se résout à lui confesser la mort de son mari. Cette confession a lieu sur un escalier à la gare, alors qu'Igor a organisé le départ d'Assita en Italie, chez des parents. Cette rencontre finale prend tout son sens si on la rapporte à ce que Luc Dardenne note dans son journal à la date du I9 janvier 1996, à savoir que Levinas est mort pendant le tournage de La Promesse et que le film doit énormément à la lecture de ses œuvres, et plus particulièrement à son interprétation du face-à-face et du visage comme discours premier. Il ajoute: «Tout le film peut être vu comme une tentative d'arriver enfin au face-à-face» (p.56).

La scène finale de la confession met en place une rencontre qui fait résonner la conception éthique de Levinas. Cette scène ménage une pause dans le film. Igor est au bas de l'escalier, Assita deux marches plus haut. La confession se dit tout d'abord hors cadre tandis qu'on ne voit qu'un plan statique du dos d'Assita, la tête de son bébé à la limite inférieure de l'écran. La caméra à l'épaule et l'activité constante qui portaient le film jusqu'alors font soudain relâche. Trop honteux pour s'adresser à elle en face, Igor se confesse au dos d'Assita avant qu'elle ne se retourne. La tête baissée d'Igor se relève ensuite pour soutenir son regard, mais c'est alors elle qui regarde au loin, puis s'éloigne. L'image finale est fixée plein cadre tandis qu'ils disparaissent tous les deux au loin. Une promesse est toujours faite au futur, quelle qu'elle soit, et Igor la tient en ne réitérant pas la violence meurtrière mais plutôt en révélant la mort d'Hamidou. Le mouvement de la caméra fait ici une pause suffisamment longue pour que la spécificité de ces instants ultimes soit perçue par contraste avec le reste du film. Je qualifie cette rencontre finale de levinassienne du fait de l'iconoclasme propre à la scène comme aux travaux esthétiques du philosophe, que les Dardenne n'évoquent d'ailleurs jamais mais qui occupent une place centrale dans leur cinéma.

Dans un texte de jeunesse, "La réalité et son ombre $»^{12}$, Levinas présente l'œuvre d'art comme ce qui ombre et immobilise l'être, l'enlève au temps et le suspend pour l'éternité, sans possibilité d'établir une quelconque relation au futur, pourtant si important dans son système éthique. Plus généralement, la plasticité des images y est immédiatement détruite,

I2. E. Levinas, «La réalité et son ombre», Les Temps modernes, nº38, 1948, p. 771-789. 
interdisant en cela que le visage devienne jamais objet ou image pour notre regard. L'iconoclasme est aussi présent dans le journal de Luc Dardenne lorsqu'il mentionne à plusieurs reprises le désir de déconstruire les sempiternelles images cinématographiques (plan/contre-plan, plan subjectif, plan d'ensemble). La quête de nouveauté formelle brise les codes et tente de tout recommencer à zéro tout en reconnaissant que c'est impossible. De même, afin d'éviter la sédimentation traditionnelle de la forme, les Dardenne emploient des comédiens néophytes. Qui plus est, c'est par leur manière de filmer le dos de leurs personnages qu'ils poursuivent leur entreprise de démolition des idées préconçues au cinéma. Ce n'est pas l'image littérale d'Igor et d'Assita l'un en face de l'autre qui établit un lien avec la pensée de Levinas mais plutôt le moment qui précède, qui montre leurs dos à la caméra lorsque Igor se confesse. C'est cette posture contorsionnée qui permet de rendre cinématographiquement la rencontre éthique levinassienne, laquelle ne se limite pas à La Promesse.

\section{En filmant le corps}

Après La Promesse, la méthode de tournage des Dardenne cherche à saisir l'évolution des personnages. Le cadrage agence les vies fictives sans jamais les contenir, les corps en dépassent les limites plutôt que d'être contenus par les plans. Après La Promesse, l'esthétique formelle de leurs films devient plus radicale (telle l'extrême compression des plans de Rosetta) tandis que l'intrigue semble créée par les plans acérés sur les agissements des personnages. Souvent, la caméra suit ces derniers sans que la cinématographie en devienne une simple formule convenue. Il s'agit de filmer à hauteur de personnages, d'acteurs, de corps. Les vues plongeantes (par exemple dans L'Enfant lorsque Sonia et Bruno sont filmés montant les escaliers par une caméra située deux marches plus haut) n’interviennent que lorsque l'espace de la scène le requiert. Tirés par les personnages, nous nous sentons avec eux et cependant légèrement derrière, notre point de vue donnant souvent sur leur dos. Cette façon de filmer le dos rappelle Levinas. Luc Dardenne déclare avoir voulu cadrer le dos de Rosetta pour «briser l'image déjà vue et sue par le spectateur» (p.I29). Le film débute par un plan à l'épaule montrant une Rosetta en colère, habillée d'une blouse blanche et d'un chapeau d'ouvrière, sortant en trombe de l'usine dont elle vient de se faire licencier sans raison. Dans Le Fils plus que dans tout autre film le dos du personnage principal est un point focal de référence. 
L'histoire débute dans l'obscurité et sur le dos d'Olivier Gourmet. La caméra est si proche de ses vêtements qu'elle cache la lumière et nous empêche de cerner le lieu de la scène: un mouvement opaque, à peine visible, ne peut être précisé. Lorsque le générique s'achève, nous sommes enveloppés de sons que nous percevons plus clairement que les images, même s'ils n'ont pas non plus de point d'ancrage - jusqu'à ce que la caméra se retire et révèle que nous sommes en train d'écouter les bruits d'un atelier de menuiserie. C'est là qu'Olivier travaille et réinsère de jeunes délinquants en œuvrant à leur formation professionnelle. Notre premier regard, toujours de derrière à ce stade, est témoin de son refus de prendre le garçon dont il lit le dossier. Il annonce au directeur qu'il ne saurait l'accepter tout en semblant obsédé par ce jeune homme. Nous le suivons tandis qu'il déambule dans les couloirs en tentant d'apercevoir le candidat apprenti. Olivier nous est présenté comme étant plutôt équilibré, mais la cinématographie suggère le contraire. Il porte une ceinture lombaire qui semble couper et recentrer son corps torturé par les émotions.

Nous apprenons plus tard que Francis, le jeune garçon, a tué le fils d'Olivier lors d'une tentative de vol de voiture cinq ans auparavant. La fascination tourmentée d'Olivier pour le tueur de son fils l'amène finalement à le prendre en formation dans l'atelier. Son impassibilité dans la manipulation des outils de son métier, lesquels pourraient servir à un second meurtre pour venger la mort du fils, produit une violente ambiguïté à travers tout le film. Jusqu'à la scène finale, la relation forcée d'Olivier et Francis demeure incertaine, entre pardon et vengeance. Comme lors de la confession dans La Promesse, la révélation par Olivier qu'il est le père de la victime est faite à brûle-pourpoint et dans le dos de Francis, filmé dans un angle oblique permettant de voir les deux hommes, ce qui perturbe l'équilibre de leur relation, et la confuse poursuite qui s'ensuit culmine dans une strangulation filmée latéralement. Mais Olivier ne peut mener sa tentative de meurtre-vengeance à terme. Son incapacité à tuer le meurtrier de son fils offre à Francis un futur possiblement différent de son passé et Olivier, épuisé, s'assied finalement aux côtés de cet improbable fils de substitution. Après la révélation et la tentative d'étranglement, le film s'achève sur l'image d'Olivier et Francis en train de fixer en silence leurs planches de bois. Cette séquence est très pauvre en dialogues. Ici comme ailleurs, c'est dans la façon de filmer les mouvements corporels et les gestes que s'articule une pensée éthique. Cependant, le désir de s'ouvrir vers l'altérité demeure inséparable d'une dimension verbale. Le face-à-face levinassien rapporte la pensée aux mots d'une façon 
qui correspond à ce que nous observons dans les mouvements corporels des personnages des frères Dardenne, comme dans l'organisation plastique de leurs images filmiques.

L'impératif éthique conceptualisé par Levinas engage le sujet depuis une position de hauteur verticale plutôt que depuis une position d'extériorité spatiale $^{\mathrm{r} 3}$, et l'impératif est verbal. Une telle adresse depuis une position de hauteur pose en même temps la transcendance de la relation éthique. Dans les films par contre, l'angle de prise de vue du corps n'incarne pas littéralement l'orientation éthique levinassienne. En choisissant fréquemment de centrer sur le dos de leurs protagonistes, donc d'y attirer notre regard, les Dardenne déplacent la topographie du corps vers le bas plutôt que vers le haut: du visage au dos. Et cela s'opère sans qu'il y ait déperdition dans l'intensité de la dimension éthique de la rencontre. L'impératif du visage levinassien s'exprime par les mouvements du corps dans l'espace diégétique, et aussi dans ceux du film (à la fois en termes de cinématographie et de montage). Et c'est le dos qui s'avère être le point d'origine de ce mode d'adresse. Luc Dardenne parle d'ailleurs du dos de Gourmet comme s'il s'agissait d'un visage: «comme si ce dos, cette nuque parlaient» (p.I29). Cela rejoint ce que Judith Butler dit de cette partie de l'anatomie lorsqu'elle commente l'article de Levinas «Paix et proximité». ${ }^{\mathrm{I}}$ Interprétant ce texte, dans lequel il est dit que des parties du dos pleurent, reniflent et crient, Butler soutient que la description levinassienne du corps nous porte aux limites de la traduction langagière. Elle interprète alors le visage levinassien comme étant essentiellement le son qui "précède et limite l'exposition du sens sémantique». ${ }^{\text {Is }}$ De la même façon le corps d'Olivier dans Le Fils nous porte à la limite de ce qui peut être vocalisé, tout en nous communiquant sa confusion comme sa douleur. Le dos de Gourmet est éloquent dans son silence tout en rendant plusieurs de ses gestes illisibles par leur ambiguïté. Les frères Dardenne façonnent ainsi une éthique à la frontière du monde visible et, qui plus est, ils désignent le lieu où la pensée, comme l'expression linguistique, atteint ses limites. Le dépassement des codes cinématographiques, le questionnement de l'image et cette façon de filmer le corps sont autant d'étapes dans la désarticulation du vocabulaire filmique traditionnel.

I3. E. Levinas, Totalité et infini, ouvr. cité, p. I85.

I4. E. Levinas, «Paix et proximité» [1984], Altérité et transcendance, Paris, Fata Morgana, I995, p. I38-I5O.

I 5. J. Butler, Precarious Life. The Powers of Mourning and Violence, New York, Verso, 2004, p. I34. 
L'adresse verbale au visage contribue pleinement à briser la réduction totalisante de l'altérité au même: elle déplace la pensée et la contreeffectue. Pour Levinas, la pensée est toujours liée au pouvoir, et l'ontologie se définit sur cette base comme philosophie du pouvoir. ${ }^{16}$ Étudiant la troisième Méditation cartésienne dans Totalité et infini et la façon dont elle pose la pensée comme relation à quelque chose de purement extérieur à elle - Dieu, l'infini ${ }^{17}$, dans le sens où il est possible, selon Descartes, d'avoir une idée de Dieu mais où cette dernière ne saurait avoir d'origine humaine -, Levinas assimile l'éthique à une pensée qui pense plus que ce à quoi elle pense: une sorte de pensée qui excède toute tentative de la contenir - et en cela ressemblante aux corps filmés par les frères Dardenne, excédant perpétuellement le cadre. Chez Levinas, la pensée rencontre sa première limite lorsque le langage l'excède: "Aborder Autrui dans le discours, c'est accueillir son expression où il déborde à tout instant l'idée qu'en emporterait une pensée» (ibid., p.43). Développant ce questionnement éthique des fondations ontologiques de la philosophie dans Autrement qu'être ou au-delà de l'essence, Levinas tente de développer une critique performative de l'être: le Dit comprend des énoncés susceptibles de confirmation ou d'interrogation, alors que le Dire se rapporte à une situation sous-jacente qui expose les énoncés en question (et la personne qui les prononce ou y répond) à l'altérité. Le langage éthique résulte du labeur du Dire, lequel rompt avec les discours statiques du Dit. En amont de toute structure ontologique de signification, Levinas affirme les capacités signifiantes de la sensibilité, trouvant ainsi du sens hors l'ontologie. Cette sensibilité se caractérise comme essentiellement vulnérable, et cette vulnérabilité même est ce qui signifie. ${ }^{18}$ Usant d'un vocabulaire explicitement levinassien, mais sans jamais citer Autrement qu'être, Luc Dardenne se demande comment lui et son frère pourraient projeter différents types d'images dans leurs films: «Pourrions-nous projeter une image qui serait comme le visage d'autrui, aussi vulnérable et intense par-delà sa plastique?» (p.29). Ce qui est à rapporter à une interrogation ultérieure: «Les images de nos films peuvent-elles provoquer une faille, un trou dans ce qui est?» (p. IO8). Ainsi à l'instar de Levinas, les deux cinéastes recherchent une porte de sortie leur permettant d'échapper à ce que l'on pourrait nommer l'impasse d'être, et offrant à l'altérité la possibilité de surgir. Cette sor-

I6. E. Levinas, Totalité et infini, ouvr. cité, p.37.

I7. Ibid., p. IO; p.39-42.

I8. E. Levinas, Autrement qu'être, ouvr. cité, p. IO4. 
tie de secours se déploie à travers le corps humain et ses images, attestant les limites de la pensée comme de la parole. Cependant, leurs films testent aussi les limites de l'existence incarnée, ce qui réarticule la relation entre corps et esprit proposée, entre autres théoriciens du cinéma, par Gilles Deleuze. Selon ce dernier, le corps, plutôt que d'être un obstacle à surmonter pour pouvoir penser, nous entraine profondément vers l'impensé de la pensée. C'est la vie qui est cachée à la pensée, et le corps nous force à la pensée. ${ }^{19}$ Pour les Dardenne, ce n'est pas le corps du sujet pensant qui nous entraîne vers les limites de la pensée, mais plutôt le corps de l'autre, de quelqu'un d'autre. L'impensé rencontré ici est la relation éthique ellemême, plutôt que la vie sentie par le corps du sujet pensant. Ce dernier est ainsi ouvert à l'autre d'entrée de jeu, immédiatement.

Le syntagme "au dos de nos images», titre du journal de Luc Dardenne, attire l'attention sur quelque chose que nous ne voyons pas d'ordinaire. Tel le point aveugle du corps, le dos de l'être humain requiert et appelle un autre point de vue pour pouvoir être vu. Dans les films des Dardenne la caméra invisible crée cette position d'altérité que Daniel Frampton assimile à la conscience du film. ${ }^{20}$ Luc Dardenne évoque quant à lui la notion de «corps-caméra" pour décrire la symbiose parfaite entre le caméraman Benoît Dervaux et l'assistant réalisateur Amaury Duquenne sur le tournage de L'Enfant (p. 175). Dans Autrement qu'être Levinas présente la responsabilité originale comme étant figurée par un marquage corporel, plus précisément sur ou dans la peau : «Dans la responsabilité en tant qu'assigné ou élu - du dehors, assigné comme irremplaçable - s'accuse le sujet dans sa peau - mal dans sa peau. $\gg^{2 \mathrm{I}}$ Marque corporelle invisible, mais indélébile, la responsabilité levinassienne est exigeante et douloureuse, fardeau que l'humanité porte depuis des temps immémoriaux, à sa surface plutôt que dans des profondeurs psychologiques. Parallèlement, c'est par une insistance sur la surface des corps et des objets que les films des Dardenne tentent de dépasser le grain, de le traiter comme une membrane afin de filmer «l'affleurement du corps invisible dans le corps visible» (p. I33). Le mouvement inconscient d'Olivier vers le meurtrier de son fils est un exemple de la sphère cachée que les Dardenne s'attachent à discerner sur le corps visible. Par-delà le monde perceptible, qui, dans le sens de l'observation quasi documentaire, semble le sujet le plus évident

I9. G. Deleuze, L'Image-temps. Cinéma 2, Paris, Seuil, I985, p. 246.

20. D. Frampton, Filmosophy, Londres, Wallflower Press, 2006, p. I47.

2 I. E. Levinas, Autrement qu'être, ouvr. cité, p. I67. 
de leurs films, affleure une autre dimension, immatérielle et invisible dans ses propriétés et pourtant bel et bien présente. La rencontre éthique entre les corps est ce qui rend possible la perception de cette dimension autre.

\section{L'âme du film}

Les frères Dardenne ont été les premiers à prendre de la distance avec les discussions théoriques sur la pensée et l'incarnation au cinéma, en choisissant de rapprocher leurs films de la notion levinassienne d'âme humaine plutôt que de corps ou d'esprit. Ils ne se sentent pas concernés par le devenir de l'âme après la mort: leurs personnages sont indubitablement de ce monde-ci. S'exprimant sur Le Fils, Luc Dardenne explique que l'impossibilité de tuer le jeune homme est le vrai sujet du film, plutôt que la notion de pardon, et il résume cette impossibilité comme "[1]'âme humaine selon Levinas» (p.95). Plus tôt dans son journal, il paraphrase la référence de Levinas à l'âme humaine dans Difficile liberté: essais sur le judä̈sme ${ }^{22}$ en la sécularisant et en la prélevant du contexte judaïque propre à cette œuvre. Il transpose ensuite ses remarques à la question de l'art:

L'art est reconnu par beaucoup comme une manifestation de notre possibilité d'immortalité, comme dur désir de durer, comme anti-destin. Pourrait-il être une modalité de l'institution de l'impossibilité de tuer? Pourrait-il ouvrir à cette âme qui se découvre comme impossibilité de donner la mort à autrui ? (p. 42)

Cette conception contraste implicitement avec la capacité prêtée au cinéma de connaître l'après-mort. La définition que donne André Bazin de l'ontologie de l'image photographique comprend cette dernière comme ce qui embaume un instant temporel et ainsi sauve le sujet photographié d'une seconde mort spirituelle. Il étend cette notion de préservation de soi aux images cinématographiques et décrit comment elles capturent la durée et momifient le changement. ${ }^{23}$ Le mouvement comme le temps cinématographiques sont alors pensés en termes de passage éternel et d'immortalité. La conception de l'art développée par les frères Dardenne est sensiblement différente. Leur façon d'interroger l'observation réaliste et leur dette évidente à l'égard du cinéma documentaire les rapprochent du Bazin

22. E. Levinas, Difficile liberté: essais sur le judaïsme, Paris, Albin Michel, 1963.

23. A. Bazin, "Ontologie de l'image photographique» [1945], Qu'est-ce que le cinéma? Paris, Cerf, 2002, p. 9-17. 


\section{Du visage au dos}

de I945, fût-ce tardivement. Cependant, leur intérêt pour la dimension spirituelle est moins à rapporter à une survivance de l'individu photographié ou filmé qu'à une insistance sur la relation qui se fonde sur la survie de l'autre. Leur conception du cinéma en termes d'âme plutôt que d'esprit ou de corps individuels ne se réduit pas à un intérêt thématique ou diégétique. Sur le plan formel, la conjonction de Levinas et des Dardenne suggère plutôt que l'autre est toujours là par l'esprit et empêche le film d'être identifié à un sujet solipsiste, que ce soit en termes d'esprit, de corps, ou d'une combinaison des deux.

Cette dimension spirituelle est filmée à travers le prisme de références judéo-chrétiennes sans pour autant être totalement ancrée dans une religiosité donnée. Le physique et le métaphysique demeurent étroitement liés. À propos de L'Enfant et de son rapport aux œuvres précédentes, François Bégaudeau note le thème chrétien qui parcourt tout le corpus et présente ce film comme "plein de ces glissements d'une cause matérielle à un effet moral». ${ }^{24}$ Sans suivre une telle logique mais en conservant ce passage du matériel au moral, cette même connexion apparaît aussi dans d'autres films, plus spécialement dans Rosetta, où la survie économique est un thème essentiel à travers lequel la dimension éthique et spirituelle de l'existence peut être traitée. Luc Dardenne cite Levinas : "La vie spirituelle est essentiellement vie morale et son lieu de prédilection est l'économique." Ce constat de Levinas est aussi celui de notre cinéma » (p.70). Cette façon de situer le spirituel au sein du matériel suggère la présence de ce qui demeure insaisissable ou intouchable dans le tangible, point de contact avec la pensée de Levinas.

La Rosetta éponyme est engagée dans une quête d'emploi quotidienne et se bat pour être socialement acceptée, tout en supportant une mère alcoolique et l'absence du père. L'adolescente est d'entrée de jeu la mère de sa mère, la réprimandant pour sa légèreté mais la louant aussi, pour son ouvrage. Elle survit sans aucune protection de l'État ou de sa famille décomposée. Rosetta se bat pour faire partie du système économique, refusant la position qui lui a été assignée. Lorsqu'elle est sans emploi, elle pose des pièges à poisson, vend des vêtements, remue sans cesse, comme en témoigne son souffle dont le bruit accompagne toutes ses activités. Chaque fois elle se fait renvoyer sans raison. À part sa mère, la personne la plus proche d'elle s'appelle Riquet (Fabrizio Rongione), et

24. F. Bégaudeau, "La matière, infiniment», Cahiers du cinéma, octobre 2005, p.24-26 et particulièrement p. 24 . 
vend des sandwichs depuis un camion sur la route. Il devient son ami et lui offre la possibilité de vendre des gaufres avec lui, activité relativement lucrative mais illégale, et qu'elle refuse. Le moment le plus serein qu'elle connaisse a lieu lorsqu'elle passe la nuit chez Riquet, sur un lit d'appoint. Les mots qu'elle se chuchote à elle-même au moment de s'endormir forment comme une prière séculière, à travers laquelle elle répète dans la pénombre la grammaire de la nouvelle existence sociale qu'elle entrevoit. À la première personne, puis à la seconde, elle dit son nom, note qu'elle a un emploi, un ami et une vie normale, et qu'elle ne tombera pas dans un trou, avant de se souhaiter bonne nuit à elle-même. La personne qui se montre la plus chaleureuse à son endroit est pourtant celle qu'elle trahira. Prête à tout pour être régularisée dans le monde du travail, elle avertit le propriétaire du camion du petit trafic parallèle de Riquet, et s'empare de son poste. Le remplacer d'une telle manière ne lui procure aucune joie. À la fin, le film la montre capable de voir plus loin qu'elle-même.

La caravane qu'elle partage avec sa mère doit lui servir de chambre funéraire. Elle joint le propriétaire par téléphone pour l'avertir qu'elle démissionne, ce qui ressemble à un acte d'effacement social. À la différence de la Mouchette de Bresson - influence pourtant avouée du film dont l'acte final est le suicide, la tentative d'asphyxie par gaz de Rosetta est empêchée par le fait que la bouteille est à moitié vide. Elle se procure vite une autre bonbonne qu'elle pousse jusqu'à la caravane, filmée derrière l'épaule puis de face. Le bruit de la bicyclette de Riquet est audible et augmente lorsqu'il tourne autour d'elle. Elle laisse tomber la lourde bonbonne pour lui lancer du gravier, puis repart. Il ne la lâche pas jusqu'à ce qu'elle s'effondre en larmes. Dans le dernier plan du film elle semble voir Riquet pour la première fois: son bras l'aide à se relever de sa position prostrée sur le sol. L'obsession de sa propre condition - et de celle de sa mère - semble ici s'estomper tandis qu'elle laisse quelqu'un s'approcher d'elle, quelqu'un qui pourrait l'aider. C'est avec le regard en larmes de Rosetta vers Riquet hors cadre que le film s'achève. Luc Dardenne écrit que lui et son frère voulaient filmer l'inconnaissable de Rosetta à travers son regard (p. 73), la figurant ainsi d'une manière qui rappelle le visage levinassien dans son appel à joindre l'extrasensoriel, la dimension immatérielle de l'existence. Son inconnaissabilité soulève cependant une question épineuse en ce qui concerne la représentation de la subjectivité féminine au sein de l'unité familiale. 


\section{Le féminin et le roman familial}

L'élément spirituel du cinéma humaniste des frères Dardenne se joue dans un monde sans Dieu: Dieu le père est absent et rien ne le remplacera. La stable figure d'autorité du père de famille dans la sphère sociohistorique est également mise en doute dans chacun de leurs films. La figure du père évolue entre l'ogre (La Promesse), l'homme cassé (Le Fils), l'adulte irresponsable (L'Enfant), ou l'absent (Rosetta). Pour Levinas, c'est par la ligne paternelle, de père en fils, que les générations se lient et que passé et présent s'ouvrent au futur. Le statut du féminin est problématique dans un tel schéma - dans la philosophie levinassienne comme dans le cinéma des frères Dardenne. Dans Totalité et infini, c'est par l'amour érotique entre un homme et une femme que la relation au futur s'incarne dans l'engendrement d'un enfant. Le concept de caresse nomme la relation non possessive du sujet mâle à sa maîtresse, ce qui rend possible son lien à l'extérieur et au futur. Le roman familial qui sous-tend la pensée de Levinas est ici très traditionnel. Il charrie toute une histoire du confinement du féminin à l'«intérieur». Même si le philosophe affirme qu'il est concerné par la problématique du féminin - plutôt que par celle de femmes concrètes -, ce dernier n'en demeure pas moins essentiellement inscrit dans le foyer, le domestique et l'accueil des autres. Par contre, la subjectivité masculine est présentée comme mobile, apte à s'aventurer dans l'espace en général, dans l'espace éthique que l'accueillant féminin domestique rend possible et accessible.

À cet égard, La Promesse met en place l'ambiguïté du statut du féminin, tout en ajoutant à l'équation levinassienne la question des différences non sexuelles. Assita incarne à la fois une position radicalement autre face à Igor, culturellement en tant que femme immigrée du Burkina Faso, et une matriarche, plus proche de lui qu'il ne semble. La Promesse traite de l'humanisation progressive d'Igor qu'Assita rend possible par sa présence, mais en cela, elle est en quelque sorte à son service, occupant une position équivoque qui rappelle celle que Totalité et infini assigne au féminin. Dans une critique du concept levinassien de caresse, Luce Irigaray suggère que l'acte de caresser revient à agencer un futur pour le sujet masculin refusé au féminin. ${ }^{25}$

25. L. Irigaray, "Questions to Emmanuel Levinas. On the divinity of love», Re-reading Levinas, R. Bernasconi et S. Critchley éd., Londres, The Athlone Press, I99I, p. IO9-II8. 
L'intérêt des Dardenne pour l'évolution du regard masculin est notable dans d'autres ouvres. Dans Le Fils, Magali, l'ex-compagne d'Olivier, est enceinte de son nouvel amant, ce qui suggère qu'elle a été capable d'évoluer, alors que lui, non. Cependant, elle est troublée en apprenant que Francis a été libéré, puis, plus tard, s'évanouit lorsqu'elle l'aperçoit. De tels passages montrent sa vulnérabilité comme sa douleur alors que le film porte principalement sur la capacité d'Olivier à accepter l'existence de Francis. Mais Rosetta demeure la tentative la plus élaborée de filmer un personnage féminin. Les frères Dardenne révèlent qu'ils l'ont longtemps approchée comme s'ils filmaient le corps d'un homme: "Notre premier corps de femme, filmé comme celui d'un homme. On l'appelait Rosetto. $\gg^{26}$ Ses crampes d'estomac, qu'elle ne calme que par la chaleur d'un sèche-cheveux, ne sont jamais nommées comme étant spécifiques au corps féminin, bien que sa mère déclare qu'elle souffrait de la même façon. Par comparaison aux trois films précédents L'Enfant traite d'un couple homme/femme et tente de donner autant d'importance à Bruno qu'à Sonia à travers les relations différentes qu'ils entretiennent chacun avec l'enfant.

Comme La Promesse et Le Fils, L'Enfant met en jeu une relation entre père et fils. Improbable figure paternelle, Bruno (Jérémie Renier) est luimême un enfant irresponsable, apathique plutôt que clairement mauvais, et qui s'en remet sans réfléchir à un comportement délictuel pour se faire de l'argent. L'Enfant montre comment Bruno devient progressivement responsable. Sa compagne Sonia (Déborah François) est elle aussi une présence forte du film. Sa spécificité féminine, fût-elle prise dans la maternité, est notée; elle ne fait pas que servir l'autoréalisation de Bruno. Le lien maternel entre Sonia et Jimmy, l'enfant, est fort. Avec Bruno elle a une relation joyeuse, qui se fissure néanmoins lorsque, réfractaire aux responsabilités de la paternité et indifférent au bébé, il vend Jimmy pour adoption. La brusque dépression nerveuse de Sonia le pousse à essayer de défaire ce qu'il a fait. Il récupère Jimmy mais doit payer des intérêts pour le racheter. Tentant sans succès de voler un sac à main à l'arraché, lui et son complice, Steve, se cachent dans la rivière, où ce dernier est victime d'hypothermie. Tandis que Bruno cherche de l'aide, la police arrête Steve sous ses yeux. Le film le montre ensuite pousser à travers les rues le scooter cassé dont il s'était servi pour le vol, ce qui semble plus dur que

26. J.-P. et L. Dardenne, «Nos dates clés», Libération, I9 octobre 2005. 
de pousser le berceau de son fils, motif récurrent du début du film. Il finit par arriver au commissariat, où il avoue sa responsabilité sous les yeux de son jeune complice. Même si la réponse judiciaire à cette confession est l'emprisonnement, le geste final de Bruno revient à reconnaitre sa responsabilité en général, ce qui brise la structure répétitive de son histoire personnelle et est suivi d'une réconciliation émue avec Sonia. Le retour de cette dernière à la fin du film marque le retour du féminin.

À la différence des scènes d'aveu de La Promesse et Le Fils, où Igor et Olivier révèlent leurs secrets à Assita et Francis, Bruno est filmé de face plutôt que de dos. Il s'adresse à Steve de côté, pour lui rendre les clés du scooter, puis confesse à l'inspecteur être le chef du duo. Les derniers plans du film montrent Bruno pénétrant dans le parloir de la prison. Sonia (absente des scènes précédentes) l'attend et, lorsqu'il s'assied, offre de lui apporter un café du distributeur voisin. Sonia et Bruno sont filmés séparément au début, reliés par le mouvement de la caméra, assis l'un en face de l'autre. Ils sont silencieux jusqu'à ce que Bruno s'enquière de Jimmy. Les derniers plans les montrent s'enlaçant par-dessus la table et éclatant en sanglots - réaction spontanée qui les réunit tous les deux dans le cadre, réconciliés sans parole. La tristesse de la scène comme de la peine de prison dont on ignore la durée ne parvient pas à supprimer tout à fait la lueur d'espoir des larmes de Bruno et Sonia. À travers le glauque du décor comme de leur situation, quelque chose d'indéfinissable est perceptible, qu'on ne peut rapporter à leurs larmes ou à l'espace visible. L'aptitude du spectateur à être touché par la souffrance des personnages vient de ce qu'il se sent proche des deux mais ne s'identifie à aucun. La dimension éthique - immatérielle - s'incarne, ici comme ailleurs, par la capacité qu'a le film de produire un contact avec autre chose qu'un pur sujet solipsiste. Comme avec Rosetta, Olivier et Igor, nous voyons ces personnages sans pouvoir nous fondre en eux en termes identificatoires, et cette position fermement «hors la vie» des personnages est précisément le point éthique.

\section{Position de spectateur et proximité}

Chacun de ces films présente des exemples différents de ce que signifie occuper la place d'un autre. Lorsque Rosetta prend la place de Riquet après l'avoir trahi, cela peut être lu comme l'opposé non éthique de la confession de Bruno dans L'Enfant, où il prend toute la responsabilité 
de ses actes en ne laissant pas Steve payer pour lui. Le Fils comme La Promesse montrent des actes d'apprentissage par lesquels le novice apprend un métier en prenant la place du tuteur après que celui-ci lui a montré quoi faire. En insistant sur la relativité éthique en jeu dans l'acte de prendre, ou d'échouer à prendre, la place de l'autre, ces films interrogent la notion d'identification cinématographique. Théorisée par la psychanalyse, l'identification est le processus psychologique et la possibilité inconsciente de se mettre à la place d'autrui pour sentir ce que les autres ressentent. Ce qui peut suggérer un mode éthique de relation et de compréhension de ce qui meut autrui. En théorie du cinéma, plus spécialement chez Christian Metz, l'identification a lieu lorsque le spectateur voit comme le personnage - identification primaire à la caméra menant à une identification secondaire au sein de l'espace diégétique. ${ }^{27}$ Bien que Luc Dardenne explique que leur cinéma en appelle à une imagination morale du spectateur (p. I3I), c'est-à-dire qui demande de s'imaginer soi-même à la place d'autrui, la caméra n'offre jamais de position d'identification immédiate en ces termes. Dans Autrement qu'être, Levinas définit la subjectivité en termes de substitution, ce qui semble superficiellement se rapprocher de la structure de l'identification. Selon Levinas, la logique de la responsabilité est liée à celle de la signification en ce que l'«un-pour-l'autre», à travers lequel les signes représentent quelque chose et produisent du sens, devient l' «un-pour-l'autre » de la responsabilité et de la substitution, dans lequel je représente et suis responsable d'un autre être humain. Sa notion de responsabilité se rapporte cependant à plusieurs aspects de l'identification et n'est pas psychologiquement ancrée. De la même façon, le cinéma des frères Dardenne n'est pas d'empathie - à travers des chemins identificatoires classiques - mais de proximité.

Si proche de la personne filmée que se porte leur caméra, si proche soit le regard qu'ils saisissent, ou l'objet de ce regard, ils ne manquent jamais de créer une distance au sein même de cette proximité. Autrement qu'être mène moins à penser une expérience phénoménologique des choses ou des gens dans le monde qu'une proximité à eux. Le point de contact définissant cette proximité est théorisé avant le langage et comme la condition de

27. C. Metz, Le Signifiant imaginaire, Paris, UGE, I977. Cette théorie psychanalytique est critiquée par de nombreux théoriciens, mais d'une façon qui diffère de ma critique levinassienne: voir à titre d'exemple M. Smith, Engaging Characters. Fiction, Emotion, and the Cinema, Oxford, Clarendon, I995; et V. Sobchack, Carnal Thoughts. Embodiment and Moving Image Culture, Berkeley, University of California Press, 2004. 
possibilité de ce dernier: "Proximité comme dire, contact, sincérité de l'exposition; dire d'avant le langage, mais sans lequel aucun langage, comme transmission de messages, ne serait possible. $\gg^{28} \mathrm{Ce}$ que Levinas dit du langage et de la pensée a été précédemment rapporté à la désarticulation du vocabulaire filmique traditionnel. Ici, il s'agit plutôt d'en étendre la portée à une analyse de la relation du spectateur à l'image sur l'écran. La position de proximité - c'est-à-dire d'être placé spatialement presque comme un personnage sans l'être tout à fait - préserve le spectateur de l'identification pure et simple. Luc Dardenne nomme la distance entre la caméra et le personnage l'espace du «secret», ce qui confêre aux personnages une existence propre en maintenant toujours le spectateur à distance de la position du personnage (p. I3O). Les personnages s'interposent toujours entre la caméra et sa position idéale. C'est la proximité, plutôt que l'expérience, de la perception des personnages qui est cruciale ici, et la pensée levinassienne parvient à nous porter par-delà les modèles psychanalytiques et phénoménologiques. La possibilité de voir ou de sentir comme le personnage est remplacée par une proximité qui paradoxalement permet d'avoir conscience des autres personnages ainsi que de notre propre distance à ceux qui sont filmés. C'est donc la capacité à ne pas prendre la place des personnages en s'identifiant à une image qui rend possible la reconnaissance de la responsabilité. L'espace du secret nomme la différence éthique et immatérielle entre des positions qui ne peuvent jamais s'identifier l'une à l'autre. Cela explique que nous puissions être émus sans reproduire ou même partager les émotions incarnées que nous voyons sur l'écran. Située par-delà toute mimésis corporalisée, cette réponse du spectateur laisse intacte l'altérité. Réagir à l'âme de ces films, c'est donc reproduire l'impossibilité de se mettre à la place des personnages pour voir comme ils voient, sentir comme ils sentent. Cela revient à porter dans l'univers du spectateur le refus ou l'inhabilité de tuer. Comme l'écrit Luc Dardenne: «Regarder l'écran [...] ce serait: ne pas tuer» (p.42).

Les frères Dardenne échangent la mort contre la vie dans leur refus de répéter l'acte radical de suppression de l'altérité. Cependant, l'insistance sur les personnages dans l'image ne concerne pas la survie individuelle de ceux-ci, mais leur relation de dépendance les uns avec les autres, les uns des autres. La façon dont les personnages sont filmés démontre

28. E. Levinas, Autrement qu'être, ouvr. cité, p.32. 
une conscience très forte du danger de prendre la place d'autrui, et elle est propre à leur façon d'interroger le meurtre et sa prohibition. Le souci d'arrêter la répétition du meurtre littéral ou symbolique s'étend à la question de la place du spectateur devant l'image, créant ainsi un espace de responsabilité. Les frères Dardenne lisent la philosophie de Levinas dans sa relation à l'art d'une manière plus positive que ne le fait Levinas luimême, et leurs films articulent une relation entre son éthique et le cinéma que Levinas lui-même n'avait pas envisagée. Leur intérêt pour l'altérité et l'âme produit un cinéma qui crée une fissure originale dans l'ontologie du cinéma - une fissure ontologique donnant à la vie des autres un espace et une temporalité différents. C'est en définitive le corps, et son image cinématographique, qui, en articulant le passage du visage au dos, crée cette fissure. 


\section{$:::$}

\section{Le territoire de l'extase : le corps et le paysage dans l'œuvre de Bruno Dumont}

La question du corps structure l'œuvre de Bruno Dumont et doit être envisagée selon deux perspectives: les relations entre les corps et le rapport que ces derniers entretiennent au paysage. On propose ici de prendre pour point de départ une affirmation du réalisateur constatant l'échec de la sexualité: "Les corps qui s'unissent délimitent. » $^{\mathrm{I}}$ Si l'impossibilité de l'union des corps dans la sexualité est une constante de la filmographie de l'auteur, elle a cependant pour contrepoint la quête d'une fusion du corps avec la nature, survivance du mythe de l'«homme-paysage» dans la production cinématographique française contemporaine. À rebours des interprétations idéalistes de l'œuvre du réalisateur, on voudrait poursuivre l'analyse situant les enjeux politiques de cette fusion et s'interroger sur la démarche de Dumont qui consiste à réintroduire la notion de territorialité tandis que l'union du corps avec le paysage est censée abolir cette dernière. En ce sens, les corps qui s'unissent au paysage posent également une limite. Tel est le paradoxe de ce que nous proposons d'appeler le «territoire de l'extase» dans l'œuvre de Dumont. La filmographie du réalisateur tient à ce jour en cinq films. ${ }^{2}$

I. B. Dumont, entretien diffusé sur France Culture le I7 août 2007.

2. La Vie de Jésus, film sorti en I997, dépeint la vie de Freddy et de sa bande; le groupe désœuvré assassine Kader, rival amoureux du personnage principal. En 1999, Dumont réalise son deuxième film, L'Humanité: le film suit le cours de l'enquête menée par Pharaon, jeune policier, suite au viol et au meurtre d'une petite fille dont le corps a été retrouvé dans un champ. En 2003, Dumont réalise Twenty-nine Palms dans le désert de l'Ouest américain, tandis que le quatrième film du réalisateur, Flandres, sorti en 2006 , retrace le parcours d'un jeune agriculteur arraché au paysage qui lui est familier, dans le nord de la France : c'est au contact du désert dans un pays en guerre que sa violence se déchaîne de façon aveugle. Hadewijch, cinquième long-métrage de l'auteur, n'était pas sorti en salles lors de la rédaction de ce texte. 


\section{Représentation et réception: «les corps qui s'unissent délimitent»}

Chez Dumont, le commerce des corps est fondamentalement voué à l'échec. On voudrait envisager le problème à deux niveaux: la représentation de la limite séparant les corps entre eux, et, du point de vue de la réception, l'impossibilité pour le spectateur d'investir le corps de l'image projetée. Si le réalisateur reconnaît que «la sexualité, c'est le moment extraordinaire de la tentative de la fusion $»^{3}$, celle-ci est néanmoins illusoire: "Le sexe est le seul moyen de faire corps, de copuler encore et encore jusqu'à en mourir. C'est la voie tragique de la fusion, du désir à l'inaccessible $U N$ que nos corps humains fragmentés, exilés, tentent de recouvrer. »4 La frénésie des accouplements de Freddy et de son amie dans La Vie de Jésus, celle de Domino et de Joseph allongés sur le sol du salon sous les yeux de Pharaon dans L'Humanité, l'agression sexuelle dans le désert californien de Twenty-nine Palms et le viol collectif par les soldats de Flandres offrent le spectacle d'une mécanique des corps réduits à l'impuissance dans la tentative de possession d'autrui. Chez Dumont, la sexualité est une tragédie qui oppose le désir de se consumer dans la jouissance à l'impossibilité d'habiter le corps de l'autre. Si la pénétration physique est une lutte à mort de l'individu pour posséder autrui, elle se réduit à la gesticulation d'un corps solitaire. La sexualité est confinée à un territoire de l'échec: c'est en ce sens que «les corps qui s'unissent délimitent». Aussi n'est-il pas étonnant de voir le corps porter les stigmates de la division que la violence est censée conjurer. L'échec de la «petite mort » déchaîne la pulsion de meurtre. La blessure de la chair cristallise l'impossibilité de la fusion: au début de L'Humanité, Dumont filme ainsi le corps inerte d'une fillette dont on devine qu'elle a été violée puis assassinée. Dans un plan faisant inévitablement penser à L'Origine du monde de Gustave Courbet, la caméra posée au ras du sol filme l'entrejambe meurtri de l'enfant. La violence est rendue par la composition du cadre: le sexe occupe l'espace du plan et relègue le visage de l'enfant dans le hors-champ. Le paysage reprend ses droits sur le corps inanimé qui ne semble plus être celui d'un humain, mais celui d'un mammifêre livré au cycle des éléments natu-

3. B. Dumont, entretien diffusé sur France Culture le I7 août 2007.

4. Propos de B. Dumont publiés dans le dossier de presse cité par F. Garbaz, «L'Humanité», Positif, $\mathrm{n}^{\circ} 465$, novembre 1999, p. 7 , note 3 . 
rels: des insectes cheminent sur la peau et entament lentement la décomposition du corps. L'herbe, d'un vert saturé, crée un contraste avec les gouttes de sang sur la peau blême du corps. Le traitement chromatique est ici l'équivalent cinématographique d'une antiphrase, tant la couleur inverse la représentation codée d'une enfance supposément aussi tendre que l'herbe. D'un plan, le réalisateur impose au spectateur la violence de l'acte sur le mode du simple constat. Cette séquence de L'Humanité constitue la scène primitive d'un traumatisme à la fois corporel et paysager qui fait de la quête de l'unité l'enjeu des films de Dumont.

L'expérience sensorielle de la limite ne renvoie pas seulement, du point de vue esthétique, à la représentation de l'impossibilité des corps de s'unir et de la pulsion de meurtre qui s'ensuit. Du point de vue de la réception, elle a pour corollaire une délimitation du corps du spectateur et du corps de l'image en raison de la distance imposée par le style du metteur en scène. La participation affective du public consiste en effet à substituer un corps imaginaire à la conscience du corps réel dans la salle de cinéma. L'expérience du spectateur repose en principe sur une activité de fusion : il touche du regard le corps cinématique, avec pour corollaire l'occultation momentanée de son propre corps dans la salle. La série dite «proprioceptive», terme par lequel Henri Wallon désigne le sentiment qu'a le spectateur de son corps propre pendant la projection, s'efface à la faveur de la constitution d'un corps second qui se confond avec l'image projetée, c'est-à-dire avec le corps des personnages représentés sur l'écran. ${ }^{5}$ Or, c'est précisément parce que «les corps qui s'unissent délimitent», pour reprendre les termes du réalisateur, que la représentation de la sexualité dans l'œuvre de Dumont invalide toute dimension «haptique» dans le processus de réception. De même que les personnages de ses films tentent de pénétrer le corps d'autrui dans l'espoir d'une fusion, de même, le spectateur se voit refuser la constitution d'un corps cinématographique. La mise en scène crée ainsi une distance, non pour exacerber le désir du spectateur d'atteindre les corps en activité, mais bien pour le forcer à se dessaisir du corps procuratoire que lui offre en principe l'image en mouvement: le regard posé sur l'activité sexuelle est ainsi dépourvu

5. H. Wallon, Revue internationale de filmologie, $\mathrm{n}^{\circ} \mathrm{I} 3$, avril-juin 1953 . Dans Film, perception et mémoire, J.-P. Esquenazi rappelle: «[...] ce corps immobile, cloué dans le fauteuil, n'existe plus pendant la projection du film », si bien que le mouvement de l'image «aurait pour résultat d'instituer l'oubli de son corps propre pour le spectateur, ce qui lui permettrait d'adhérer au système perceptif du film, de s'intégrer aux mouvements du film» (Paris, L'Harmattan, I994, p. IO3). 
de toute participation affective. À l'exil permanent des personnages les uns par rapport aux autres correspond donc l'impossibilité éprouvée par le spectateur d'investir l'image projetée sur l'écran. En ce sens, le filmage des corps ne repose pas chez Dumont sur une activité de recognition, mais sur un processus d'évidement de l'image. L'absence de toute participation affective scelle la crise de l'image haptique, à laquelle se substitue l'œil de la pensée, ce qu'atteste l'intérêt du réalisateur pour Platon: «En vérité, l'œil de la pensée ne commence d'avoir le regard pénétrant que quand la vision des yeux commence à perdre de son acuité. $»^{6}$

Une séquence de Twenty-nine Palms inscrit dans l'espace la problématique de l'exil du corps du point de vue de la représentation et de la réception. D'un plan général, la caméra filme en plongée la piscine d'un motel, près de la route, dans le désert de Californie. Le ciel est d'un bleu limpide. Dans la profondeur de champ se dresse un paysage de moyenne montagne aride; à l'avant-plan, la piscine, dont la surface lisse est à peine troublée par les mouvements de David et Katia; à l'arrière-plan, le paysage qui sera plus tard le théâtre de l'agression sexuelle subie par David sous les yeux de sa compagne. Le plan fixe s'installe dans la durée tandis que l'orage menace. Tel un prédateur, David s'approche lentement de sa compagne, située à l'autre bout de la piscine; elle se refuse aux avances de son compagnon ( «On doit partir à cause de l'orage»), mais il lui impose un acte sexuel dans la piscine. La séquence instaure un contraste entre la violence du désir dont la femme est l'objet, et la présence du paysage immobile qui semble indifférent à l'action. L'imperturbable présence de la montagne à l'arrière-plan réduit la sexualité à de vaines gesticulations dans lesquelles les personnages noient leur solitude. La présence du paysage vide la scène de tout affect. Sous ce rapport, cette scène peut être rapprochée de l'interprétation que livre Louis Marin du tableau de Poussin Pyrhame et Thisbé peint en I65I, représentant le drame amoureux des personnages dans un paysage englouti par la tempête. Le texte de Marin porte une attention particulière au contraste entre l'impétuosité des éléments, l'intensité des liens qui unissent les amants, et la présence d'un lac dont la surface à peine ridée, au centre du tableau, impose une sérénité inattendue. Pour Marin, il s'agit d'un lac d'indifférence qui ressemble à "un miroir d'eau calme, celui de Narcisse, mais où, loin d'en mourir par stupéfaction de son propre désir de voir, le regard du sage se contemple

6. Platon, Phédon, cité par B. Dumont dans Bruno Dumont, S. Ors, P. Tancelin, V. Jouve éd., Paris, Dis-voir, 200I, p. I4. 
dans la figure de son oil, en contemplant, apaisé, l'œuvre de sa peinture, dans sa représentation "indifférente" à ce qu'elle représente».7 En ce sens, la piscine de Twenty-nine Palms est l'œil de la pensée qui évide le corps dans le plan. ${ }^{8}$

\section{La crise des vecteurs de l'identité spatiale}

Chez Dumont, la question des rapports entre le corps, la limite et le paysage entretient un rapport étroit avec la question de l'identité. Les films du réalisateur révèlent en effet la faillite des vecteurs traditionnels de l'identité spatiale : plus que la conscience de soi dans un lieu, le réalisateur livre le spectacle de la crispation du corps qui, faute de posséder autrui par la sexualité, s'inscrit dans les limites d'un territoire. ${ }^{9}$ Les pratiques identitaires traditionnelles apparaissent comme autant de vestiges condamnés à disparaitre: dans La Vie de Jésus, l'harmonie municipale de Bailleul peine à cristalliser l'identité collective. Certes, les musiciens font le tour de la commune, tout comme les processions religieuses faisaient le tour de la paroisse pour y inscrire une identité collective. Certes, l'harmonie municipale est représentée comme le lieu d'une affiliation à un groupe qui soude les regards: Freddy est fier d'en faire partie et sa petite amie valide ce sentiment par son regard bienveillant. Pourtant, Dumont filme la scène avec ironie: avant d'entamer son parcours dans un vaste paysage de plaines par une journée d'hiver, il montre les musiciens près d'un troquet dont le nom, "Le Coin perdu», scelle l'impossibilité de créer un sentiment d'appartenance dans une communauté privée de lieu. Flandres présente de façon similaire la faillite des vecteurs traditionnels de l'identité collective: la séquence de l'appel sous les drapeaux montre longuement le camion des autorités militaires et les conscrits en offrant à l'arrièreplan une représentation stéréotypée du village français avec son clocher.

7. L. Marin, «La description du tableau et le sublime en peinture », Sublime Poussin, Paris, Seuil, I995, p. IO4.

8. On pourrait également comparer la représentation du corps dans le paysage dans Twentynine Palms à la représentation du temps géologique dans le désert, en particulier dans le cinéma de John Ford; sur ce point, voir L. Cortade, «Le vieillissement, la filiation et le paysage dans She Wore a Yellow Ribbon de John Ford", Contre Bande, n I3, 2005, p. 45-52.

9. Dans son analyse de La Vie de Jésus, T. Lounas remarque que « les corps ne se définissent que par la place qu'ils occupent. C'est-à-dire qu'à quelque endroit qu'ils se trouvent, ils ne signifient rien de plus que l'appartenance à un même territoire » (Cabiers du cinéma, $\left.\mathrm{n}^{\circ} 5 \mathrm{I} 4, \mathrm{I} 997, \mathrm{p} .76\right)$. 
Le service militaire n'est pas évoqué comme le creuset d'une conscience identitaire collective, mais comme une intrusion du monde extérieur sur le territoire de la commune et un déracinement qui entraîneront le déchaînement de la violence des personnages. L'incommunicabilité entre les corps est en outre perceptible à travers les nombreuses occurrences des «non-lieux» dans la filmographie de Dumont. ${ }^{\circ}$ Dans L'Humanité, un plan général cadre la gare de Lille filmée de façon impersonnelle: le bâtiment se dresse, comme une violence faite au paysage. À Londres, Pharaon reste dans le terminal pour interroger les témoins du meurtre. Au travers des baies vitrées, il aperçoit deux hommes en train de se battre; une plongée montre le corps des individus noyés dans un aplat couleur ardoise: pour Dumont, la violence sourd de l'absence de lien organique au paysage. Dans l'usine où travaille Domino, les vêtements de protection, le plastique et les tuyaux rutilants imposent au corps un exil dont les rares échanges verbaux entre collègues ne peuvent l'extraire. Dans le même film, le musée devient également un non-lieu: les tableaux sont échoués dans un cimetière d'œuvres d'art.

La faillite des vecteurs identitaires traditionnels et le rôle des non-lieux scellent le constat de la crise du lien social et de la communauté, laquelle se dissout dans un agrégat d'individus qui défendent leur territoire. Dans La Vie de Jésus, les jeunes hommes sillonnent le paysage environnant dans une tentative de s'approprier l'espace par le mouvement de leurs motocyclettes et de leur voiture; dans L'Humanité, Joseph, le meurtrier de la fillette, passe ses journées au volant et noie son ennui dans l'expérience de la vitesse; dans Twenty-nine Palms, David et Katia sont agressés verbalement par le conducteur d'une voiture rouge qui dévale à toute allure et leur lance: «C'est notre rue, ici! Dégagez!» La mise en scène est en effet émaillée des signes d'un cloisonnement entre l'intérieur et l'extérieur de la ville. La représentation du malaise social et le repli sur le territoire se fait par l'absence de plans établissant un lien spatial entre les habitants et le paysage. Les corps sont reclus dans le huis clos de leur habitation: l'absence d'articulation entre la ville et le paysage distend le lien social." Chez Dumont, l'exil intérieur des personnages se cristallise particulière-

IO. M. Augé, Non-lieux. Introduction à une anthropologie de la surmodernité, Paris, Seuil, 1992.

I I. L'idée selon laquelle il n'est de lien social qu'à la faveur d'une articulation entre la ville et le paysage rural est notamment développée, pour ce qui est du Japon, par A. Berque dans son ouvrage Du geste à la cité: formes urbaines et lien social au Japon, Paris, Gallimard, 1993. 
ment sur la fonction assignée aux portes et aux fenêtres. Les habitants restent sur le seuil de leur maison: ils semblent monter la garde devant le territoire de leur domus. Les fenêtres sont systématiquement des obstacles à la circulation de la lumière. Elles produisent l'effet d'une lacération dans le décor et fragmentent l'espace en invalidant tout rapport de la communauté au paysage. Dans L'Humanité, qu'il s'agisse du commissariat ou de l'hôpital psychiatrique, les fenêtres s'interposent entre l'individu et les éléments naturels, les carreaux étant systématiquement translucides, voire couverts de barreaux. Les symboles de la République sont arborés dans le bureau du commissariat; le portrait officiel du président de la République Jacques Chirac est accroché près de la porte, à l'intérieur du bureau du commissaire supervisant l'enquête de Pharaon. Première photographie officielle représentant un chef de l'État dans un contexte bucolique, en l'occurrence, sur la pelouse de l'Élysée, le portrait montre le président posant devant le palais ; à l'image des habitants de Bailleul qui tuent le temps sur les marches du seuil de leur porte, il défend un espace qui semble être le sien. De même, la Déclaration des droits de l'homme et du citoyen ainsi que le drapeau tricolore cristallisent bien l'identité nationale dans le commissariat, mais leur position près d'une fenêtre aux vitres translucides pose le problème de la valeur des symboles républicains qui ne sont plus que des signes vidés de leur sens car confinés dans un huis clos. Dans L'Humanité, la pratique de l'enfermement dans l'institution psychiatrique est un sevrage de la matrice paysagère, réduite à sa portion congrue. Le végétal y est méticuleusement taillé et zébré par de larges allées quadrillant une nature qui n'est désormais plus qu'un «espace vert» séparant des unités abritant les patients. La mise en scène des limites et du calfeutrage domestique a pour pendant le rôle joué par les téléviseurs, antithèses de la fenêtre ouverte sur le monde, qui imposent à la perception de l'espace une distance déréalisante. Le petit écran absorbe l'activité potentiellement violente des pensionnaires de l'institution: c'est un outil de coercition. De façon similaire, dans La Vie de Jésus, le téléviseur est allumé de façon quasi permanente dans le troquet tenu par la mère de Freddy; il absorbe la sociabilité qui échoit traditionnellement au café, si bien que le fils reproche à sa mère de regarder une course de vélo à la télévision au lieu de se déplacer pour assister à l'événement sportif qui se déroule pourtant dans les environs. La télévision s'attaque à la présence des corps réunis dans un même lieu et devient le centre de gravité autour duquel se dressent les limites spatiales du café, devenu le territoire de la mère de Freddy. C'est pourquoi le café est chez Dumont un territoire où 
se pratiquent le racisme et l'exclusion. La xénophobie de certains habitants de Bailleul se lit clairement dans l'espace du film: Kader se trouve dans le café avec sa famille et parle arabe, ce qui provoque les injures des musiciens de l'harmonie municipale. Alors que les Maghrébins refusent la violence et finissent par sortir, un plan montre un drapeau français au seuil de la porte, tandis qu'un musicien joue le début de La Marseillaise à la trompette. La ville frappe d'ostracisme celui que l'on appelait jadis le «horsain», c'est-à-dire l'étranger, celui qui n'est pas du «pays».

\section{«Une recherche éperdue de l'harmonie, c'est-à-dire de la fonte»}

Au constat d'échec du commerce des corps et des vecteurs traditionnels de l'identité collective débouchant sur la défense effrénée du territoire, Dumont oppose la quête de l'unité entre le corps et le paysage. Si l'homme est condamné à la régression dans la territorialité, c'est avant tout parce qu'il est sevré du grand corps originel dont il issu et avec lequel il cherche à s'unir. Le cinéma est en effet pour le réalisateur «une recherche éperdue de l'harmonie, c'est-à-dire de la fonte ». ${ }^{12}$ On sait que la peinture de paysage, contemporaine de l'émergence de l'individu à la Renaissance, a ouvert la voie à une vision anthropocentrée de l'espace dont la perspective a été l'expression plastique. ${ }^{\mathrm{I3}} \mathrm{Si}$ la notion de paysage en Occident a eu pour corollaire une dissociation entre le point de vue d'un sujet et l'objet de sa perception, Dumont abolit la distinction entre le corps percevant et le paysage : «Le cinéma est un art mystique. [...] La mystique, c'est cette espèce de perception de l'unité. $\gg^{14}$ La problématique du corps se déplace de la surface à la profondeur; dans ses notes de travail sur La Vie de Jésus, le réalisateur écrit: "Je savais que j'aspirais à filmer l'intérieur. Le dedans. Dans le ventre. Et qu'il faudrait peindre la terre. La terre et les arbres. $\gg^{15}$

Chez Dumont, l'esthétique de la fonte et de la fusion constitue une survivance du mythe de l' 'homme-paysage $»^{16}$ que le réalisateur rend

I2. B. Dumont, Bruno Dumont, ouvr. cité, p. I8.

I3. On se reportera sur ce point à l'introduction de l'ouvrage de M. Collot, Paysage et poésie du romantisme à nos jours, Paris, Corti, 2005.

I4. B. Dumont, entretien diffusé sur France Culture le I7 août 2007.

I5. B. Dumont, "Notes de travail sur La Vie de Jésus", Positif, n 440, octobre 1997, p. 58.

I6. J'emprunte cette expression au catalogue de l'exposition L'Homme-paysage, A. Tapié, J. Zwingenberger éd., Paris-Lille, Somogy - Palais des Beaux-Arts de Lille, 2006; voir en particulier J. Zwingenberger, "L'histoire du paysage anthropomorphe. Le corps, géographie du monde», p. 52-75. 
visible à l'écran par un style reposant sur les ressources du cadrage et du montage: "Le scope me contraint à toujours garder une harmonie entre

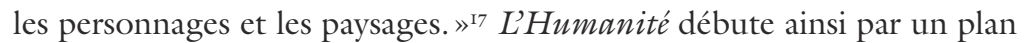
général représentant en contre-plongée le profil d'une colline au sommet de laquelle un arbre se détache en contre-jour. La croupe sombre du paysage pondère le cadre et crée un contraste avec le ciel d'ardoise hivernal. Le plan fixe s'installe dans la durée: aucun mouvement ne vient troubler le regard contemplatif que pose le réalisateur sur l'immobilité du paysage. Un individu finit par apparaittre sur le bord gauche du cadre: il s'agit de Pharaon. L'éloignement de la caméra par rapport au corps qui surgit dans le plan produit un contraste avec le cadre sonore: si le plan général rend visible la silhouette du personnage dans la distance, la bande-son fait l'effet d'un gros plan en donnant à entendre les respirations de l'homme. En privilégiant la durée et l'intégrité du plan dès le début de son film, Dumont construit le corps de l'image en dotant celle-ci des valeurs esthétiques de la continuité : aucun mouvement, aucune coupe franche ne vient rompre l'esthétique de la durée réelle. Par son mouvement, le corps est un trait d'union entre les limites du cadre (le changement de plan n'intervient que lorsque le corps disparait par le bord droit). Le paysage s'offre dans la temporalité d'un cheminement dans l'espace, qui s'oppose à la connaissance abstraite et scientifique du géographe surplombant l'espace de son regard cartographique. Le corps cerné par l'horizon est en butte à l'impossibilité de tout point de vue englobant: il est dans le paysage. ${ }^{18}$

Dumont s'inscrit dans la lignée de la phénoménologie de l'espace d'Erwin Strauss que Jean-Marc Besse commente en ces termes:

Le paysage, en tant qu'il est paysage originaire, paysage de la fusion ou de la communication originaires de l'homme et du monde, précède donc toute orientation et tout repère. Le paysage est désorientation radicale, il surgit de la perte de tout repère, il est une manière d'être envahi par le monde. ${ }^{19}$

I7. B. Dumont, dans Positif, n 465 , 1999, p. I3.

I8. Sur ce point, Dumont s'inscrit précisément dans la catégorie du "paysage-expression" établie par A. Gardies; cette catégorie désigne une «forme d'osmose, voire de fusion, qui s'établit entre personnage et paysage. [...] C'est mon regard de spectateur, épousant ou non celui des personnages, qui va, sur la base des propositions filmiques, lire la mélancolie, la solitude, la quiétude, la douceur ou la violence qui sourdent du paysage » (A. Gardies, «Le paysage comme moment narratif», Les Paysages du cinéma, J. Mottet éd., Seyssel, Champ Vallon, p. I48).

I9. Voir E. Strauss, Du sens des sens. Contribution à l'étude des fondements de la psychologie, Grenoble, Jérôme Million, 1989, chap. VII, ainsi que le commentaire de J.-M. Besse dans «Entre géographie et paysage, la phénoménologie», Voir la Terre. Six essais sur le paysage et la géographie, Arles, Actes Sud, 2000, p. I2I. 
En effet, dès le premier plan de L'Humanité, Dumont annonce ainsi une ligne directrice de son œuvre : le paysage est le lieu du lien et de la fusion. Du soin porté par le réalisateur au cadrage, on ne saurait pour autant déduire un éventuel dogme de l'intégrité du plan. La suture du corps et du paysage se constitue en effet à la faveur d'un lien dynamique: pour Dumont, le planséquence présente l'inconvénient d'être statique, non pas tant à cause de l'immobilité de la caméra que de l'absence de lien entre plusieurs plans. Or, pour le réalisateur, «l'immobilité est l'œuvre de l'esprit, son invention: elle est l'écueil du cinéma, sa vanité. Le corps (masse taiseuse et inanimée) est la matière du cinéma, son énergie ${ }^{20}$ La caméra n’est pas censée enregistrer le constat d'une harmonie préexistante, inhérente au monde, car c'est dans l'articulation des contraires que l'harmonie devient possible. La question de la fusion du corps et du paysage repose donc sur le montage, véritable «tentative de fabriquer l'harmonie. [...] par le montage, j'essaie de retrouver une continuité ». ${ }^{2 I} \mathrm{Ni}$ le corps ni le paysage ne sont des entités; ils ne doivent être appréhendés qu'à la faveur du mouvement, de l'articulation et la jonction: il faut donc, selon les termes du réalisateur, «résider à nos nœuds.$^{22}$ On remarquera avec intérêt que cette conception du mouvement et de l'immobilité du corps dans le paysage reprend l'une des caractéristiques du lieu définies par Marc Augé, et dont la mythologie grecque avait cerné l'importance: l'articulation entre le proche et le lointain. ${ }^{23}$

Chez Dumont, la fonction du montage est d'élaborer des carrefours spatiaux, ce qui fait du paysage le lieu privilégié d'une telle articulation, car, comme le souligne Besse, "les paysages sont des points de passages, multiples et répétés, du présent des possibles qui viennent, dans une vie

20. Bruno Dumont, ouvr. cité, p.I4.

2I. Entretien diffusé sur France Culture le I7 août 2007.

22. Bruno Dumont, ouvr. cité, p. I8.

23. Dans Non-lieux, ouvr. cité, M. Augé identifie les trois caractéristiques qui définissent selon lui le lieu: l'identité, l'articulation et l'historicité. Du point de vue anthropologique, cette définition du lieu recoupe en partie la conception grecque de l'espace, en particulier telle qu'elle affleure dans le couple mythologique formé par Hestia et Hermès, comme le remarque J.-P. Vernant dans «Sur l'expression religieuse de l'espace et du mouvement chez les Grecs» [1963], Mythe et pensée chez les Grecs, Paris, La Découverte, 1996. Pour ce qui est du nord de la France, on remarquera que cette conception du lieu est attestée du point de vue historique : à rebours de tout régionalisme, la conscience du lieu repose en effet sur une articulation entre le local et le lointain, comme le montre par exemple la pratique de la «marche des Incas» dans le nord de la France au XIXe siècle; voir sur ce point l'analyse de S. Gerson, «Town, nation or humanity? Festive delineations of place and past in Northern France, ca. I825-1865", The Journal of Modern History, $\mathrm{n}^{\circ} 72$, septembre 2000, p. 628-682. 
dans le monde, et qui appellent à la décision ». ${ }^{24}$ Dumont privilégie donc l'articulation entre un plan qui représente le sujet percevant et un plan subjectif qui représente le paysage. Ce dernier n'est pas une objectivation du paysage matérialisant la distinction entre la res cogitans et la res extensa, mais au contraire la quête de la fusion du corps et du paysage. La contemplation du "paysage-expression » débouche alors sur un saut qualitatif correspondant à ce qu'André Gardies appelle le "paysage-catalyse». ${ }^{25}$ Nous pouvons en analyser trois exemples dans l'œuvre de Dumont.

La séquence de finale de La Vie de Jésus montre ainsi le jeune homme littéralement happé par la verdure : torse nu, il fixe le ciel et le soleil. L'extase paysagère du personnage est rendue par l'angle de la caméra - une plongée - tandis que les plans de détail sur son corps, les insectes et la verdure font de lui un homme-paysage. À l'inanité des mots supplée la musicalité de la voix qui exerce un rôle déterminant dans le choix des acteurs par le réalisateur. On sait que Dumont prête attention aux sons naturels de la voix de ses personnages: «Le balbutiement, c'est le son du flux. » $^{26}$ L'harmonie entre Freddy et le paysage est surtout palpable dans le travail de montage et le plan subjectif représentant le ciel: Freddy devient ce qu'il voit. En se laissant incorporer par le paysage, il rédime sa faute:

L'histoire de Freddy sublime le mal qui est en chacun de nous. Notre saloperie. Un paysage. Un paysage avec des arbres. Des gens. Les corps - du sexe, du sang, de l'amour, de la violence. Les cœurs simples. Freddy. Kader. Des gars de la campagne... Aller à l'unisson. Faire chuter à terre. Sauver Freddy sincèrement. Dans un fossé, Freddy, sa face contre la terre. Tout est calme ici. Tout est lent. Il pleure. Miné. Il s'allonge, regarde les cumulus. [...] Son repentir. Il geint et on dirait qu'il prie; qu'avec les grimaces de sa souffrance, ses gémissements, il balbutie, du fond de son ventre, pardon. [...] Torse nu, agenouillé. Sa consomption. Une chrysalide. Le visage a presque disparu. Son image, éminemment sensible, douloureuse, chrétienne, magnifiquement simple. Sanctifiée. Il pleure, et c'est infini. ${ }^{27}$

Le paysage est le pivot de l'extase par laquelle le corps s'absorbe et reçoit l'absolution. Dans L'Humanité, deux séquences mettent également en

24. J.-M. Besse, «Dans les plis du monde. Paysage et philosophie selon Péguy», Voir la Terre, ouvr. cité, p. I52.

25. "Mis en présence d'un certain type de paysage, dans des conditions favorables, le personnage subit une transformation»; le paysage est alors "une composante de la diégèse susceptible de produire ou de favoriser la transformation d'une autre composante de cette même diégèse » (A. Gardies, «Le paysage comme moment narratif», art. cité, p. I49).

26. Entretien diffusé sur France Culture le 17 août 2007.

27. B. Dumont, "Notes de travail sur La Vie de Jésus», art. cité, p. 59. 
évidence le rôle du montage et du plan subjectif dans la constitution d'un lien organique entre le corps et le paysage. Dans le commissariat, pendant l'interrogatoire d'un trafiquant de drogue, une série de plans représente le visage de Pharaon qui regarde par la fenêtre; un plan subjectif montre une arrière-cour et un jardinet, le policier regarde alors le trafiquant, il s'approche silencieusement de ce dernier, puis le sent, à la façon d'un animal. Par cet acte qui mobilise la vue, l'odorat et le toucher, le corps s'affranchit de l'expérience de la limite, qu'elle soit spatiale (la fenêtre est, comme nous l'avons vu, un obstacle qui s'impose à un corps sevré du paysage) ou morale (le policier incarne un ordre et une morale à laquelle déroge le délinquant). Le montage et les plans subjectifs ont ici pour fonction de nouer un lien qui unit le sujet au paysage. Le personnage du policier établit un contact instinctif au monde, par opposition à la partition de l'espace qui caractérise les représentations de la ville et des habitats. Il ne s'agit plus de représenter le solipsisme du sujet dans une territorialité vouée à l'échec, mais de rétablir l'harmonie fondamentale entre le corps et le paysage pour absoudre la faute. À propos de Pharaon, Dumont déclare : "C'est un contemplatif. Il est en regard permanent sur les paysages, sur les gens. Les gestes qu'il fait sont assez rares, mais il prend sur lui. Je crois que c'est quelqu'un qui somatise. ${ }^{28}$ Ainsi peut-on interpréter la séquence du jardinage, l'une des rares au cours desquelles Pharaon esquisse un sourire qui déroge à son habituelle impassibilité. Dans le jardin qu'il cultive, une série de plans représente sa main qui touche la terre lourde et sombre; la caméra cadre son visage, suivi par un plan subjectif représentant le paysage. Pharaon absorbe la souffrance du monde et accède à un état extatique: son corps entre littéralement en lévitation. Cette union exerce une influence déterminante sur la conduite du récit puisqu'elle s'affranchit de la limite séparant les corps dans l'échec de la sexualité: au plan représentant le sexe de la fillette assassinée succède, à la fin du film, un plan représentant le sexe de Domino. En ce sens, la fusion avec le paysage est véritablement un retour à l'unité et à l'« origine du monde». À l'impossibilité de la fusion des corps et du sentiment d'appartenance par le biais des vecteurs identitaires spatiaux traditionnels se substituerait donc un dépassement de la notion de territoire dont l'unité retrouvée avec le paysage serait le pivot. Pour Dumont, il n'y a de corporéité que paysagère.

28. B. Dumont, dans Positif, $\mathrm{n}^{\circ} 465$, I999, p. I2. 


\section{La fusion du corps avec le paysage : une reterritorialisation}

L'œuvre de Bruno Dumont a fait l'objet de lectures faisant volontiers abstraction de la fonction politique du paysage dans la culture visuelle. ${ }^{29}$ On voudrait ici défendre l'idée selon laquelle la question de la fusion du corps avec le paysage doit être envisagée d'un point de vue historique, en particulier par rapport au questionnement identitaire dont la production cinématographique française de la fin du $\mathrm{XX}^{\mathrm{e}}$ siècle et du début du $\mathrm{XXI}^{\mathrm{e}}$ présente plusieurs indices.

Revenons pour cela au début de L'Humanité: Pharaon poursuit sa course à travers le paysage. Son regard est hypnotisé par l'horizon, la caméra suit d'un travelling latéral le mouvement de son visage, tandis qu'il marche à vive allure sur les labours; il finit par trébucher. Son visage clair est en contact direct avec la terre sombre. Son regard fixe le donne pour mort; après plusieurs secondes d'incertitude, le spectateur le voit finalement regagner sa voiture. Assis au volant, il tourne la tête en direction du champ qu'il vient de quitter. Un plan subjectif montre alors le soc rouillé d'une charrue qui semble abandonnée. Ce plan cristallise l'ambivalence du rapport de Dumont au paysage. Le corps à corps paysager illustre le thème de la fusion, en particulier dans le plan du visage posé contre terre. Or, la terre est ici associée à l'outil qui en pose les limites. D'un point de vue anthropologique, la charrue et le sillon délimitent en effet le territoire..$^{\circ}$ On peut se demander si le regard que pose Pharaon sur la terre et la charrue correspond à un désir de fusion avec le paysage dépassant l'échec de la sexualité - et par extension, celui de la limite -, ou bien au contraire à la nostalgie du territoire perdu. On saisit alors la grande ambiguïté de l'œuvre du réalisateur dans le rapport qu'il établit entre le corps et le paysage. Certes, Dumont semble poser un regard distancié, si ce n'est critique, sur les tentatives vaines et désespérées du

29. Pour une approche spiritualiste de l'œuvre de Dumont, voir par exemple l'analyse de T. Clark, «Bruno Dumont, le sacré et notre expérience de la violence», Parachute, nº I23, 2006.

30. Le récit de la fondation de Rome ainsi que l'étymologie mettent en évidence une filiation entre les mots désignant le sillon, le manche recourbé de la charrue, et la ville. Urvum désigne le manche recourbé de la charrue. Urvo signifie «tracer le sillon d'enceinte d'une ville» et semble ainsi appartenir à la même famille que Urbs désignant Rome. Le récit de la fondation mythique de la ville, tel qu'il est présenté par Tite-Live dans son Histoire romaine (livre I, 6-7), rapporte que Romulus a marqué l'enceinte de l'Urbs en traçant un sillon qu'il était interdit de franchir, mais que Remus a néanmoins franchi, ce qui lui a valu d'être tué par son frère. 
corps de se forger une identité territoriale. Pourtant, l'étude de l'union du corps avec le paysage doit s'inscrire dans une histoire des représentations de l'imaginaire de l'espace, y compris d'un point de vue politique et social. On voudrait alors formuler l'hypothèse suivante : la dissolution du corps dans la pure sensation paysagère s'inscrit dans le sillage des nombreuses occurrences du motif de la fusion dans le cinéma français à partir des années I980 sur fond de questionnements identitaires.

L'absorption du corps par l'espace urbain ou paysager connaît un certain essor depuis le début des années I980, comme le montrent le cinéma dit du «look», expression par laquelle on désigne le cinéma français postmoderne, ainsi que les films patrimoniaux dits de la "nostalgie», le plus souvent des fresques historiques ou des adaptations de romans français. Ces deux courants témoignent des rapports étroits entre le corps et le décor. Les personnages de fiction se placent en effet sous la coupe d'un lien matriciel à l'espace : ils se cloîtrent dans des lieux clos et sécurisants, s'acharnent à vouloir «faire partie des murs» ou à forger une identité qui s'inscrit dans un parcours initiatique paysager. Les espaces protecteurs dominent dans les films français de cette période: on pense par exemple au loft sans fenêtres de Diva (Jean-Jacques Beineix, I98I), à la vie souterraine dans le ventre du métro parisien dans Subway (Luc Besson, I985), à l'immersion totale du corps du nageur jusqu'à la mort dans Le Grand Bleu (Besson, 1988), à l'austère retraite bucolique de monsieur de Sainte-Colombe dans Tous les matins du monde (Alain Corneau, I99I), ou encore à la quête mystique d'Yves Montand à travers la forêt dans IP5. L'île aux pachydermes (Beineix, 1992). ${ }^{\text {II }}$ Ces films témoignent d'une évolution de la sensibilité, privilégiant les situations fusionnelles du corps avec l'espace. Le cinéma du «look» et de la «nostalgie» constituent une réponse à la crise du lien affectif et identitaire de la société française dont le «jeune cinéma français» des années I990 est par ailleurs un symptôme. ${ }^{32}$ On remarque avec intérêt que le cinéma dit de l'«héritage» pri-

3I. Pour cette période, on pourra notamment consulter le livre de M.-T. Journot, Le Courant de "l'esthétique publicitaire» dans le cinéma français des années 1980. La modernité en crise: Beineix, Besson, Carax, Paris, L'Harmattan, 2004.

32. Le «jeune cinéma français» des années I990 est un «témoignage de phénomènes de déliaison sociale: troubles identitaires, décompositions familiales, instabilités professionnelles, difficultés d'intégration, exclusions [...]. La représentation de la déliaison semble bien avoir pour corollaires la description nostalgique des attachements, ainsi que la dramatisation des attachements. Les distensions et les ruptures des liens sociaux vont de pair avec la réaffirmation - fût-elle désenchantée, voire désespérée - du besoin de lien familial, affectif, amoureux» (F. Vanoye, dans Le Jeune Cinéma français, M. Marie éd., Paris, Nathan, 1998, p. 56). 
vilégie quant à lui les représentations qui renforcent le sentiment identitaire du spectateur en satisfaisant l'" horizon d'attente paysager» par des représentations stéréotypées de l'espace national français. ${ }^{33}$ Le paysage fait dans ce cas l'objet d'une essentialisation présentant les attributs traditionnels de la «francité»: ainsi du paysage provençal dans Jean de Florette et Manon des Sources, d'après l'œuvre de Marcel Pagnol (Claude Berri, 1986). De même, la représentation de Paris dans Le Fabuleux Destin d'A mélie Poulain (Jean-Pierre Jeunet, 200I) réactive le chromo de la sociabilité citadine à la française, tandis que la représentation de la campagne dans Être et avoir (Nicolas Philibert, 2002) célèbre une utopie éducative que le cadre bucolique de la France éternelle présente comme une vérité intangible épargnée par les affres de la ville et de la mondialisation. L'évacuation de la politique à la faveur de l'extase paysagère atteint son paroxysme dans le film que Robert Guédiguian a consacré aux derniers jours de François Mitterrand, Le Promeneur du Champ-de-Mars (2005). Le film montre dès le début le rôle joué par le paysage dans la constitution d'un corps national; l'hélicoptère présidentiel survole la Beauce, tandis que le chef de l'État cite Charles Péguy en évoquant «le chapelet de meules» qui s'offre à sa vue : «Comment dire mieux l'enracinement mystique?» demande, admiratif, le président. La Beauce est un corps, un de ces «lieux de mémoire» qui charpentent la perception que les Français peuvent avoir de leur identité, sous la bénédiction de celui qui incarne la nation. Dans la séquence suivante, une visite à la cathédrale de Chartres montre le président au contact des gisants de marbre, qu'il touche pour se confronter à sa mort qu'il sait prochaine. Le réalisateur révèle ici la bicorporalité du président: d'un côté le corps de chair périssable, de l'autre le corpus mysticum du roi, qui, selon l'adage médiéval, «ne meurt jamais».34 La proximité de ces deux séquences montre que la figure du président, tout comme les champs célébrés par Péguy, incarnent la permanence de l'identité nationale essentialisée. À la fin du film, tandis que le président s'est retiré pour vivre ses tout derniers jours, un long panoramique filme un arbre, symbole de l'essence et de la continuité du pouvoir, par-delà

33. Il s'agit dans ce cas de ce qu' A. Gardies appelle le "paysage-fond», lequel «participe de l'ancrage référentiel du monde diégétique. Pour cela, bien souvent, il s'organise de manière à être reconnu par le spectateur en privilégiant les composantes attendues» ( « Le paysage comme moment narratif», art. cité, p. I45).

34. Voir sur ce point les travaux d'E. Kantorowicz, Les Deux Corps du roi, Paris, Gallimard, I989, et de R. E. Giesey, Le roi ne meurt jamais: les obsèques royales dans la France de la Renaissance, Paris, Flammarion, 1987. 
le passage de vie à trépas du président. Le film de Guédiguian s'achève donc sur cette transformation du corpus mysticum de la nation dans le paysage, ce que la voix off du journaliste qui consigne les confessions de l'illustre personnage avait compris dès le début: «Le corps du président était devenu notre corps à tous, un corps national. »35

L'interprétation de l'extase paysagère comme l'expression d'une quête d'identité et de territoire mérite encore plus d'être considérée si on l'inscrit dans le temps long de l'histoire des représentations du paysage, avant l'invention du Cinématographe. Si le XVII ${ }^{\mathrm{e}}$ siècle fut celui de la division entre la res cogitans et la res extensa, le siècle suivant s'avéra être celui de l'émergence d'un rapport fusionnel entre le corps et le paysage, ce dont témoigne l'évolution de la littérature et de la peinture au XVIII ${ }^{\mathrm{e}}$ siècle. L'œuvre de Rousseau, qu'il s'agisse des Confessions, de La Nouvelle Héloïse ou des Rêveries du promeneur solitaire, constitue un exemple représentatif de la «fusion totale entre la conscience et le paysage» qui, selon Michel Collot, caractérise la sensibilité préromantique. ${ }^{36}$ La littérature du XvIII ${ }^{e}$ siècle se caractérise par une dissolution du corps dans un cadre bucolique, à telle enseigne que «l'expérience du paysage, dans ses moments les plus intenses, est une véritable ex-stase. Tout se passe comme si le sujet sortait de luimême pour s'étendre à tout l'espace environnant, en une sorte d'ubiquité qui peut être heureuse ou vertigineuse» (ibid., p.46). Comme l'a montré Michael Fried, c'est également au cours du XvIII ${ }^{\mathrm{e}}$ siècle qu'a été élaborée la fiction de l'absorbement, pour ce qui est de la peinture. Celleci doit être envisagée selon deux perspectives: du point de vue figuratif, avec la représentation d'un corps qui semble s'abstraire lui-même dans la scène représentée (d'abord chez Chardin, puis chez Greuze), et du point de vue de la relation esthétique élaborée par un spectateur oublieux de son corps propre à mesure qu'il souhaite rentrer dans l'image. ${ }^{77} \mathrm{Si}$ le lien fusionnel avec le paysage touche à son terme avec le triomphe de la peinture de David, il est intéressant de remarquer qu'il réapparut autour des

35. Voir sur ce point L. Cortade, «The spatial evacuation of politics: landscape, power and the monarch in Robert Guédiguian's The Last Mitterrand», Yale French Studies, $n^{\circ}$ II5, mai 2009, p. 67-79.

36. M. Collot, Paysage et poésie..., ouvr. cité, p. 25.

37. Le pacte tacite qui repose sur l'occultation du peintre et l'inexistence du spectateur suppose l'absence de témoins, de sorte que ce n'est pas le peintre qui sort de sa toile, mais bien le spectateur qui y pénètre, comme l'indiquent les textes que Diderot consacre à son expérience esthétique face aux toiles de Loutherbourg, Le Prince, Vernet, Robert ou Chardin, dans les Salons de I763, I765 et I767. Voir sur ce point M. Fried, La Place du spectateur, traduction C. Brunet, Paris, Gallimard, I990. 
années I830, avec l'émergence d'une peinture française de paysages à la fois romantiques et réalistes. Dans la monographie qu'il a consacrée à Gustave Courbet, Michael Fried évoque ainsi la «quasi-fusion corporelle de la peinture et du spectateur ${ }^{38}$, à la faveur de l'aspect anthropomorphe des paysages du peintre. L'historien d'art compare ainsi le sujet de L'Origine $d u$ monde, œuvre peinte en I866, à un carnet d'esquisses de I840 représentant l'entrée sombre d'une grotte, puis aux différentes versions de $L a$ Source de la Loue, respectivement peintes en I863 et I864, et dont la composition semble annoncer L'Origine du monde, œuvre à laquelle Dumont fait lui-même référence au début et à la fin de L'Humanité. À cet égard, on peut avancer que l'homme-paysage chez Dumont s'inscrit dans la lignée de l'anthropomorphisme paysager de Courbet.

Or, les représentations picturales du paysage revêtent une fonction politique dans la culture visuelle française. Au XVIII ${ }^{\mathrm{e}}$ siècle, les tableaux de Joseph Vernet ne sont pas de simples paysages, mais constituent un inventaire géographique de la France dans un siècle marqué par un cosmopolitisme intellectuel et des échanges commerciaux intenses en Europe et dans le monde. L'essor de la peinture de paysage au cours du XIX ${ }^{\mathrm{e}}$ siècle est quant à elle l'expression d'une quête identitaire "où se ressource la permanence nationale ».39 Jules Castagnary, critique d'art représentatif de la mouvance républicaine quarante-huitarde opposée à l'Empire, a clairement établi la fonction de la peinture de paysage dans l'affermissement de l'identité nationale:

Par le paysage, l'art devient national [...], il prend possession de la France, du sol, de l'air, du ciel, du paysage français. Cette terre qui nous a portés, cette atmosphère que nous respirons, tout cet ensemble harmonieux et doux qui constitue comme le visage de la mère patrie, nous le portons dans notre âme. ${ }^{40}$

La peinture de paysage nourrit ainsi une «mémoire des origines du monde, une France d'avant la France» (ibid., p. 459).

La fusion du corps dans le paysage chez Dumont ne saurait être réduite à une approche spiritualiste ou phénoménologique: elle constitue une

38. M. Fried, Courbet's Realism, University of Chicago Press, I990, p.216 (traduction L. Cortade).

39. F. Cachin, «Le paysage du peintre», Les Lieux de mémoire, P. Nora éd., t. II : La Nation, vol. I, Paris, Gallimard, 1986, p. 439. On lira également avec profit le livre de F. Walter, Les Figures paysagères de la nation. Territoire et paysage en Europe: $X V I^{e}-X X^{e}$ siècle, Paris, EHESS, 2004.

40. J. Castagnary, cité par F. Cachin, «Le paysage du peintre», art. cité, p. 476. 
survivance des rapports entre l'homme et la nature tels que la sensibilité préromantique du XVIII ${ }^{\mathrm{e}}$ siècle, puis le romantisme du XIX ${ }^{\mathrm{e}}$ siècle les a forgés. Cette filiation revêt une signification à la fois esthétique et politique : de même que la valorisation de la peinture de paysage au XIX ${ }^{\mathrm{e}}$ siècle fut le travail des républicains afin d'asseoir l'identité territoriale et politique de la France, de même, les représentations d'un rapport fusionnel à l'espace dans le production cinématographique depuis les années 1980 ne peuvent être dissociées du questionnement identitaire qui oriente les enjeux politiques de la société française à partir de cette période. L'absorption du corps par le paysage ne consiste pas chez Dumont en un dépassement de l'idée de limite sur le mode spiritualiste : il révèle au contraire la prégnance d'une frontière qui protège l'homme-paysage des intrusions du monde extérieur. Dumont procède en fait à un déplacement de la notion de territoire dont la fusion de l'homme et du paysage serait le symptôme. À l'aune de ce paradoxe, on mesurera la grande ambiguité de la portée politique de l'œuvre du réalisateur. 


\section{Images sans organes / récit sans télos: Carlos Reygadas et Gus Van Sant}

Dans son ouvrage fondateur, De la figure en général et du corps en particulier. L'invention figurative au cinéma, Nicole Brenez développe la catégorie de «figure cinématographique », qui, tout étant constituée du corps, le dépasse, par exemple sous la forme de "personnages sans personne» ou de «corps sans support», car, écrit-elle, «en rabattant le corps organique sur les aperçus corporels proposés par le cinéma, on refuse à celui-ci l'ensemble de ses puissances figurales, ses capacités d'abstraction, sa propension à l'allégorie, ses inventions figuratives, ses diverses aberrations et son pouvoir de prévision ». ${ }^{.}$L'intérêt d'un tel concept est qu'il met en rapport la notion de corps comme puissance - de dé- ou de reconstruction - avec le pouvoir qu'a le cinéma de produire des récits singuliers et inattendus. Dans la perspective ainsi ouverte, l'une des questions de fond s'agissant du corps cinématographique devient: comment habite-t-il un récit tout en restant exempt d'une quelconque prédétermination téléologique? La réponse que je voudrais proposer ici est qu'il y parvient en demeurant toujours à saisir, en échappant continuellement à toute reterritorialisation définitive, en courant très vite ou, au contraire, en ne bougeant plus, en se fixant en une immobilité paradoxale, à la lisière de l'image, du cadre.

I. N. Brenez, De la figure en général et du corps en particulier. L'invention figurative au cinéma, Bruxelles, De Boek, I998, p.32. Dans un travail proche de celui de N. Brenez, P. Dubois isole la notion de figural comme «tout ce qui, dans une image, subsiste, une fois que l'on a enlevé en elle le figuratif (c'est-à-dire le motif référentiel, sa part iconographique) et le figuré (le(s) sens second(s), sa part rhétorique et iconographique) - mais qui reste encore figurable»; venant du "dedans de l'image elle-même », le figural est "toujours au travail dans le corps même de l'image, dans la chair vive de sa visualité » (P. Dubois, «La question du figural», Cinéma. Art(s) plastique(s), P. Taminiaux et C. Murcia éd., Paris, L'Harmattan, 2004, p.5I-76 et particulièrement p.65). 
Le corps cinématographique peut faire cela, au sein du plan par exemple, dans le déploiement d'un geste - ou encore comme pur montage: de Dziga Vertov à Michelangelo Antonioni ou John Cassavetes, les exemples, magnifiques et variés, abondent. À leur étude, le but de ce chapitre est de comprendre comment l'on passe d'un corps dans l'image cinématographique au corps de telle image, et comment ce dernier est une occurrence frappante de cette surface que Gilles Deleuze et Félix Guattari ont appelée, après Antonin Artaud, le corps sans organes. Ce programme requiert d'abord d'identifier ce que je nommerai une parenté théorique entre, d'une part, l'art cinématographique conçu comme esthétique constructiviste ${ }^{2}$ culminant dans la production d'un artefact plastique (la mise en scène, la cinématographie, le montage et le son conjurant au final à une image), et, d'autre part, l'informe natura naturans, la force dés-organ-isée/sante qu'est un corps. Le corps de l'image, plutôt que l'image du corps, sera donc étudié ici en relation avec ses tendances non anthropocentristes pour voir comment les deux, corps et image, procèdent d'une force générique - faite de rapports intensifs - plutôt que d'une forme préétablie, et de la sorte reproblématisent la question de la représentation.

Pour mener à bien ce programme, j'examinerai d'abord le corps comme pure productivité, tel que l'a pensé Deleuze. ${ }^{3}$ Je lierai ensuite ce concept à la notion de cadre, et de cadrage ${ }^{4}$, étudiant comment, au cinéma, le corps cadré, c'est-à-dire l'image du corps, se trouve paradoxalement à même de manifester ce qui, dans le corps, résiste et redétermine le cadrage d'opération purement technique (syntaxique) en mode sémiotique inédit (sorte de

2. J'emploie ce terme au sens de la philosophie deleuzienne et non dans celui développé par les théoriciens et artistes russes des années 1920, qui isolent l'aspect géométrique pur des formes du réel qu'ils agencent ensuite pour constituer des œuvres. Tandis que le constructivisme russe voit dans les lignes, les plans et les angles de productions sculpturales, picturales, architecturales ou graphiques l'expression d'une essence de l'univers (grâce à une sobriété à l'opposé de l'esthétique baroque par exemple), le constructivisme ontologique et esthétique deleuzien auquel je me réfère généralise la constructibilité non seulement aux formes mais aussi aux signes, aux significations, et aux êtres eux-mêmes; voir J. Game, «Le virtuel deleuzien : cogito pour un moi dissous», BARCA : poésie, politique, psychanalyse, $\mathrm{n}^{\circ} \mathrm{I} 5$, novembre 2000 , p. 57-80.

3. Chez ce dernier le productivisme - qui se confond avec le constructivisme et le constructionisme - est le jeu du devenir au sein de l'immanence pure: «[...] l'immanence est un constructivisme» (G. Deleuze, Pourparlers, Paris, Minuit, I990, p. 199). Du point de vue de la pensée, c'est la théorie non essentialiste rapportant le réel et le sens (l'être) à l'instance perpétuellement ouverte, c'est-à-dire instable, d'actes sans sujet, d'effets sans cause linéaire.

4. "L'image cinématographique, existant dans le temps, est d'ailleurs idéalement équipée pour incarner visiblement la mobilité, virtuelle ou actuelle, du cadre, qui est le cadrage » (J. Aumont, L'Image, Paris, Armand Colin, 2005, p. II6). 
corps sans organes sémiotique). Enfin, m'attachant à deux films en particulier, Bataille dans le ciel (Carlos Reygadas, 2005) et Elephant (Gus Van Sant, 2004), je tenterai de montrer comment l'image du corps ainsi redéfinie peut révéler une double corporéité inscrite dans un rapport d'homonymie : corporéité du corps et corporéité de l'image. Pour résumer: loin d'être simplement une forme idéale représentée dans ou par un film - en une sorte de platonisme appliqué au cinéma -, ou un thème instrumentalisé aux fins de la représentation cinématographique d'un télos - dans une perspective aristotélicienne -, le corps cinématographique me semble pouvoir être une sorte de productivité transcendantale, une force de décodage à même de susciter ce que j'appellerai une image sans organes (sans cadre prédéterminé, ni montage préétabli, ni rythme préécrit, etc.). Mon principal angle critique étant la question du cadre et mon corpus se constituant des deux films précités, mon objectif sera de soutenir que dans le cinéma créatif, un sens frais, loin de dériver d'un pur récit, ne peut au contraire que procéder d'un devenir de l'image, de son corps, et ainsi raconter une histoire où la vie est à l'œuvre, par opposition à de pesants signifiés prédéfinis s'encastrant les uns dans les autres au rythme de lois génériques. ${ }^{5}$ Un thème fondamentalement nietzschéen sera ainsi à l'œuvre: non pas la vie contre le sens, pris sous la figure du récit ou de la narration, mais la vie par-delà le sens, avant, pendant et après lui, le transperçant, le dépassant, le réagençant. Ici encore la violence de la Vie constituera l'horizon de la réflexion: ses idiosyncrasies comme, parfois, son idiotie, son senselessness. Mais aussi sa beauté et sa puissance créatrice. La tension entre Apollon et Dionysos ne finit jamais. Et peut-être le cinéma apparaitra-t-il en définitive comme une éthique pour cette tension sans fin, comme ce qui nous apprend à la supporter et ainsi à (la) vivre.

\section{Images sans organes: d'un air de famille théorique}

En lecteur de Bergson - et de Spinoza - Deleuze conceptualise le corps comme corporéité, c'est-à-dire non pas comme natura naturata mais

5. Contrairement à P. Dubois - "C'est par des effets de présence et non de représentation, de figuration et non de narration, que s'inscrivent en nous les traces du passage des images filmiques» («La question du figural», art. cité, p. 55) -, je n'exclus donc pas par principe la narration de ce cinéma des corps, mais cherche plutôt à comprendre comment ces deux procès - corps et narration - s'agencent en cinéma pour s'intensifier mutuellement, donc s'indéterminer et se reconfigurer aussi bien. 
comme natura naturans, force plutôt que forme, puissance plutôt que chose, procès plutôt que produit. Jamais hypostasiée une fois pour toutes, cette corporéité - par opposition à corporalité - est toujours-déjà engagée en un continuel procès de relation entre forces de déterritorialisation et de reterritorialisation. Travaillant la notion artaldienne de corps sans organes, Deleuze et Guattari tentent, dans les deux tomes de Capitalisme et schizophrénie ${ }^{6}$, d'invalider la notion d'organisme comme représentation a priori de la corporéité et comme processus de normalisation d'une matière indéterminée en unités contrôlables œuvrant à une subsomption idéaliste. Le corps sans organes est une désorganisation mais d'un genre particulier: ce qu'elle cherche à invalider est l'organe comme relevant d'un ensemble surplombant, comme part d'un tout, c'est-à-dire impliquant toujours-déjà ce tout, l'officialisant, lui vouant toute chair. Cette dés-organ-isation est en fait une dés-organism-ation du corps en une somme ouverte de singularités informelles, de vecteurs, de gradients, de magnitudes inédites. L'organisme comme forme a priori est remplacé par une série de rapports a posteriori constituant une surface virtuelle indissociable des flux qui la parcourent en s'entrecroisant: flux socio-historiques, libidinaux, politiques, etc. Pour Deleuze et Guattari, le corps sans organes - ou corporéité - est une série ouverte d'énergies en mouvement qui se rejoignent et se séparent d'une infinité de façons. Il est fait de circuits qui ne marchent pas, qui tombent en panne ou marchent en dysfonctionnant, et de trajectoires qui s'auto-engendrent. À la notion d'unité propre au concept d'organisme, le corps sans organes oppose celles d'hétéroclite et de discontinu, de parties sans lien de composition. Ces dernières ne s'inscrivent jamais dans une relation d'origine (comme chez Platon ou Freud), ni dans un télos (comme chez Aristote, Hegel ou même Marx). La corporéité s'entend alors comme une confusion essentielle, une accélération centripète, une auto-dissémination immanente, un hoquet perpétuel, une convulsion qui n'en finit pas de se (dé/re-)tisser, une constante métamorphose de l'image-corps, ni stable ni instable, mais métastable - s'extrayant du monde des formes pour rejoindre celui, présocratique, de l'être comme devenir.?

6. G. Deleuze et F. Guattari, L'Anti-Edipe, Paris, Minuit, I972, et Mille plateanx, Paris, Minuit, I980.

7. G. Deleuze, Pourparlers, ouvr. cité, p. 48. Voir A. Beaulieu, «L'expérience deleuzienne du corps», Revue internationale de philosophie, vol. 56, n' 222, 2002, p. 5II-522; R. Arsenie-Zamfir, «Pourquoi le corps sans organes est-il "plein"?», Europhilosophie, [en ligne], [URL: http:// www.europhilosophie.eu/recherche/IMG/pdf/Zamfir.pdf], consulté le 26 février 2OIO; 
À propos du cadre au cinéma, Jacques Aumont écrit dans un ouvrage récent:

Doxa: ce à quoi nous sommes accoutumés, parce qu'au fond nous avons encore l'habitude de faire une équation entre image et image photographique, c'est le cadre comme limite de l'image (de forme variable quoique assez unanimement rectangulaire): le cadre-limite. Que dire, sinon ceci : ce cadre-limite est purement accidentel; c'est ce qui appartient le plus, et le plus exclusivement, à la contingence. Il n'est pas indispensable ni inévitable de produire des images dotées de ce type de cadre; l'histoire, qui nous l'a imposé, travaille aujourd'hui à nous en éloigner ou à nous en défaire. [...] Il y a toujours eu ces deux pensées du cadre: le cadre comme acte d'attention-perception-conscience(-pensée), et le cadre comme machinedispositif-site-institution. ${ }^{8}$

C'est la prégnance de cet "accident», et ses ressources cachées, qu'il s'agit de dégager afin d'en extraire les conditions d'un usage créatif du cadrage. Dans la plus grande part du cinéma classique et traditionnel, que ce soit en Europe ou à Hollywood (beaucoup moins pour le cinéma asiatique, nous y reviendrons), le cadre et le hors-cadre, le champ et le horschamp ${ }^{9}$ ont, c'est connu, une fonction syntaxique aussi précise que simple; ils servent à structurer le récit filmique et ses significations de manière binaire selon une grille dialectique présence/absence: soit une présence absente, soit une absence présente - de choses, de personnes, de corps au sein du cadre. Comme en peinture, le cadre détermine l'espace diégétique - et tout ce qui s'y trouve - de manière idéale et a priori, l'on pourrait même dire positivement ${ }^{10}$, en travaillant le centrage et en faisant le point sur les objets cadrés, ou en signifiant ce qui ne s'y trouve pas, par exemple par décentrement (ibid., p.II6). Même en travaillant en négatif, la visée et le résultat restent le plus souvent les mêmes. ${ }^{I I}$ Dans la célèbre théorie du cadre comme cache d'André Bazin par exemple ( "Les limites

et S. Lash, "Genealogy and the body: Foucault/Deleuze/Nietzsche», The Body. Social Process and Cultural Theory, M. Featherstone, M. Hepworth et B. Turner éd., Londres, Sage, I99I, p. 256-280.

8. J. Aumont, Matière d’images, Paris, Images modernes Cinéma, 2005, p. 93.

9. Ces deux notions sont évidemment distinctes, elles désignent des espaces différents mais néanmoins contigus. Je les joins ici autour de ce qui les rassemble: la problématique du «hors", de ce qui est ou demeure hors image.

IO. "Le cadre est ce qui sépare, et d'abord perceptivement, l'image de son dehors" (J. Aumont, L'image, ouvr. cité, p. I09).

I I. C'est le cadre-limite défini par J. Aumont: «[...] ce qui manifeste la clôture de l'image, sa non-illimitation. Il est le bord de l'image, en un [...] sens non tangible, il est sa limite sensible» (ibid., p. IO8). 
de l'écran ne sont pas, comme le vocabulaire technique le laisserait parfois entendre, le cadre de l'image, mais un cache qui ne peut que démasquer une partie de la réalité $\left.\gg^{12}\right)$, le cadre classiquement conçu suppose l'existence de corps idéaux qui se trouvent être camouflés derrière ou par le cache. Le corps est alors toujours prédéterminé par son inscription, que ce soit dans la toile, en peinture, ou dans l'obscurité, c'est-à-dire derrière le cache noir, au cinéma. Dans les deux cas, le corps préexiste à son image comme à la question de sa localisation ou de son emplacement. Du coup, le cadre comme le champ sont soit l'extension des limites du corps, soit leur réservoir - ces deux fonctions impliquant elles-mêmes une conception du corps comme forme: il s'y trouve déjà tout formé. Tel un système de poupées russes s'emboîtant les unes dans les autres, tous les corps, dans les mots de Françoise Parfait, «explorent, mesurent, se heurtent et se battent avec un espace qui détermine absolument leurs mouvements et leur imaginaire ». ${ }^{13}$ L'on pourrait ajouter : et vice versa. Dans cette conception du cadre, le corps est lui-même assigné à corporalité comme on l'est à résidence: fixé, punaisé contre l'esquisse ou le contour dessiné de sa forme idéale, suggérée par le cadrage traditionnel. Cette boucle épistémologique rationalisant l'image-corps par le cadre, et réciproquement, se reproduit dans la très grande majorité des films, comme à leur insu. La classique séquence champ/contre-champ en est peut-être le format le plus commun. Comparant les réflexes iconographiques occidentaux à ceux en usage au Japon, Sergueï Eisenstein avait bien marqué, dès les années 1920, la Weltanschaunng à l'œuvre ici :

Les Japonais agissent de façon inverse. Voici une branche de cerisier, ou un paysage avec voiliers. Dans cet ensemble l'élève découpe en carré ou en rond, ou en rectangle, son unité compositionnelle. Choisit son cadrage! [...] Notre école, c'est la méthode expirante de l'organisation spatiale du phénomène devant l'objectif [...]. Et les Japonais c'est l'autre méthode, l'«appréhension » par la caméra, l'organisation par elle. On taille un morceau de réalité par les moyens de l'objectif. ${ }^{14}$

I2. A. Bazin, Qu'est-ce que le cinéma ? Paris, Cerf, 1985, p. I88. Dans le même texte on trouve aussi ceci sur le thème du cinéma (et donc de son cadrage) comme futur de la peinture: "Qui ne voit dès lors que le cinéma, loin de compromettre et de dénaturer un autre art, est au contraire en train de le sauver en lui rendant l'attention des hommes. [...] Le cinéma ne vient pas "servir" ou trahir la peinture mais lui ajouter une manière d'être» (p. I9I).

I3. La citation est extraite d'un texte intitulé "Le corps cadré, le corps contraint ", traitant d'art vidéo, mais l'on est en droit de se demander si la remarque n'est pas aussi valide (plus?) pour le cinéma: F. Parfait, Vidéo: un art contemporain, Paris, Regard, 200I, p. 198.

I4. Cité par D. Villain, Le Cadrage au cinéma. L’xil à la caméra, Paris, Cahiers du cinéma, $200 I$, p.I2. 
Mais si les arbres et les paysages sont des natures mortes, le corps humain, a priori, ne l'est pas. Immédiatement, des problèmes plus généraux en jeu dans la question du cadrage reviennent donc en force. Dans les mots de Dominique Villain:

[Cette question] renouvelle celle des limites, que les philosophes se sont posée, oscillant entre deux conceptions, à savoir, schématiquement, la conception platonicienne selon laquelle les êtres, les corps, les choses se définissent par leurs limites, et la conception stoïcienne selon laquelle les limites dépendent de l'être, les limites vont jusqu'où peut le corps, l'être, le personnage. ( Ibid.)

La taxonomie générale des signes cinématographiques développée par Deleuze dans L'Image-mouvement ${ }^{\text {I5 }}$ reprend cette même question de manière créative. Isolant d'abord deux «tendances» du cadre, «à la saturation ou à la raréfaction» (p.23) de l'image, elle le considère ensuite en lui-même, le disant soit "géométrique» - constitué «par rapport à des coordonnées choisies» ou "des variables sélectionnées», il est "composition» par «équilibre» et «invariant» (p. 24) -, soit «physique»- produit alors par le «rapport aux parties du système qu'il sépare et réunit à la fois» (p.25). Mais chacune de ces déterminations est encore passible d'une modalité particulière: le «dynamisme» ou nature processuelle, performative - «en acte», dit Deleuze - de la construction du cadre, «qui dépend étroitement de la scène, de l'image, des personnages et des objets qui le remplissent» (p.24-25). La conception dynamique du cadre physique nous retiendra plus précisément ici en ce qu'il «se construit autour de la puissance et des mouvements des corps qui l'occupent $\gg^{16}-$ et donc montre essentiellement ceci : la force d'occupation des corps plutôt que leurs délimitations formelles. Aux limites géométriques prédéfinies testant dialectiquement l'essence des corps dans l'image par la mise à l'épreuve de leur existence ${ }^{17}$, Deleuze oppose ainsi une conception du cadre comme

I5. G. Deleuze, L'Image-mouvement, Paris, Minuit, 1983.

I6. P. Marrati, Gilles Deleuze. Cinéma et philosophie, Paris, PUF, 2003, p.32. Voir aussi P. Pisters, The Matrix of Visual Culture. Working with Deleuze in Film Theory, Palo Alto, Stanford University Press, 2003, p. 55-76.

I 7. L'on pense par exemple à Alain Delon et Monica Vitti déambulant dans, sur et aux bords des cadres mondrianesques de l'architecture moderne de Rome dans L'Éclipse (I962); à Ben Gazzara contemplant la lumière matinale dans les rue de Los Angeles et à sa silhouette en smoking encadrée dans la porte de son cabaret dans Meurtre d'un bookmaker chinois (1976); à Gena Rowlands regardant fixement l'objectif et butant sur les murs rectilignes de sa chambre d'hôtel à New York dans Une femme sous influence (1974); ou encore à Catherine Deneuve assise et attendant son jeune amant entre les quatre murs 
limite quasi physiologique - mais alors, de cette physis paradoxale dont relève le corps sans organes - déterminée par la puissance des corps en présence mais les affectant directement en retour. Le cadre, par ses limites, fait alors bien plus qu'ouvrir le regard vers un hors-champ abstrait ou mystique ou encore mythique. Aucune métaphysique négative ou de l'absence n'intervient ici. Au contraire, le type de cadrage - fixe ou mobile, frémissant ou tremblant, etc. - détermine différents modes de présence du dehors au sein du dedans. À la limite, le hors-champ et le hors-cadre se confondent. La différence pure devient présence du virtuel. Le différent n'est pas l'«autre» mais le «même» lui-même. Le même est intrinsèquement différent. Ainsi le cadrage dynamique donne-t-il directement à percevoir, selon un subtil mode de sensibilité à l'image comme à ses équilibres précaires, le devenir ou la force ou encore la natura naturans qu'est un corps. En effet: comment montre-t-on une force? Par ce qu'elle accomplit? Par ce qu'elle n'est pas? Ou encore en donnant à voir ce qui est affecté par elle? À plus d'un titre rôde ici l'idée qu'un corps est beaucoup trop puissant pour sa propre forme, pour ses propres limites, et qu'il est ainsi voué, comme intrinsèquement ou logiquement, à s'excéder - par explosion ou implosion, par fonte, par morcellement, par ce qu'on voudra. Dès lors, la même chose arrive à l'image d'un corps relativement à son cadre. Ce qui ne veut pas dire que la forme du corps ou le cadre de l'image doivent jaillir hors d'eux-mêmes, mais qu'ils transforment leurs propres limites en problèmes ou en puissances plutôt qu'en délimitations figées. Montrer le corps, c'est montrer ces mille façons dont il se débat contre sa forme, ou au contraire l'accepte pour mieux la subvertir, c'està-dire le montrer autant qu'il est engagé dans un profond mouvement de devenir à un niveau transcendantal: non pas comme mouvement de quelque chose - formes, corps, individus -, mais tout entier transformé en pur mouvement, faisant sauter toute légalité dans son élan. Cet événement dans la vie des corps - l'indétermination figurale - est bien en jeu et à l'œuvre dans ce que recouvre le concept deleuzien de cadrage physique dynamique qui, est-il écrit dans L'Image-mouvement, «induit des ensembles flous qui ne se divisent plus qu'en zones ou en plages» (p. 26). Le hors-cadre comme le hors-champ y sont neutralisés, dé-typifiés, rendus abstraits: pures marches vers et en provenance du chaos. Le hors-cadre n'est maintenant rien d'autre que le tout, ce que Bergson nomme l'ou-

de son petit studio parisien, observant les plis de sa jupe, ne sachant que faire de ses mains, dans Le Vent de la nuit (1999). 
vert et Deleuze lui-même, après Joyce, le chaosmos, cosmos chaotique ou mélange métastable de chaos et d'osmose : chaos rendu consistant, consistance résistant à tout durcissement - dans la terminologie deleuzienne, à toute tendance molaire. Ce tout qui englobe le hors-champ, c'est aussi la durée bergsonienne ou l'éternel retour nietzschéen. Le cadre dynamique qui l'introduit ou le figure annihile toute détermination spatiale et temporelle : il montre (il fait voir) un hors-champ radical, un dehors à toute représentation du temps et de l'espace comme entités homogènes. C'est ce que Deleuze nomme son «aspect absolu par lequel le système clos [le plan] s'ouvre à une durée immanente au tout de l'univers, qui n'est plus un ensemble et n'est pas de l'ordre du visible» (ibid., p. 30). Comme cet absolu, il est alors ce qui introduit la durée et le tout dans l'image via les corps, c'est-à-dire ce qui les rend visibles comme forces. ${ }^{18}$ Un tel cadrage ne coupe ni n'exclut ou nie quoi que ce soit; bien au contraire, il rapporte, il ajoute. Mais ce qu'il ajoute est la puissance immatérielle du devenir qui, paradoxalement, provient des corps comme force:

Plus le fil est épais qui relie l'ensemble vu à d'autres ensembles non vus, mieux le hors-champ réalise sa première fonction, qui est d'ajouter de l'espace à l'espace. Mais, quand le fil est très ténu, il ne se contente pas de renforcer la clôture du cadre, ou d'éliminer le rapport avec le dehors. [...] plus il est ténu, plus la durée descend dans le système comme une araignée, mieux le hors-champ réalise son autre fonction, qui est d'introduire du trans-spatial et du spirituel dans le système qui n'est jamais parfaitement clos. (p.30-3I)

Par ce jeu de la durée une circulation s'instaure. Tantôt c'est l'image qui suinte ou fuit vers l'extérieur du cadre; tantôt, par la pression du chaos de l'extérieur sur les corps évoluant à l'intérieur du cadre, c'est le monde, la matière et les corps qui se trouvent reconfigurés en un suintement généralisé, en une fonte universelle. Mais montrer cela, montrer cette corporéité - et la voir - requiert justement un cadre avec ses écarts et coupures apparents. ${ }^{19}$ Les plans mettant en scène la corporéité livrée à elle-même

I 8. Cela est à rapprocher de ce que, dans un article remarquable, M.-C. Ropars-Wuilleumier nomme la "puissance d'inclusion de l'extériorité » que Deleuze - à la suite de Foucault tirerait de la pensée du dehors blanchotienne, "La "pensée du dehors" dans L'imagetemps (Deleuze et Blanchot)", Le Temps d'une pensée. Du montage à l'esthétique plurielle. Marie-Claire Ropars-Wuilleumier, textes réunis et présentés par Sophie Charlin (SaintDenis, PUV, 2009, p. 40I-4I7).

I9. Sur la manière dont Deleuze fait usage des concepts de P. Bonitzer (le «décadrage» notamment) et de N. Burch, voir D. Zabunyan, Gilles Delewze. Voir, parler, penser an risque du cinéma, Paris, Presses de la Sorbonne-Nouvelle, 2006, p. 84. 
abondent dans le cinéma moderne, et il serait tentant d'en étudier le plus grand nombre possible. ${ }^{2}{ }^{\circ}$ Faute de place, je m'attacherai ici à deux films en particulier, récents, en provenance du Mexique et des États-Unis.

\section{Mexico désert de violence: solitude et vie urbaine}

Bataille dans le ciel conte l'histoire d'un homme, Marcos, dont l'occupation est, tous les jours, à l'aube, de lever les couleurs nationales dans le Zocalo, la place centrale de Mexico. Il est de surcroît le chauffeur d'un général haut placé, dont il va chercher la fille, Anna, à l'aéroport ce matinlà. Cette dernière se prostitue pour passer le temps dans un bordel de luxe, où Marcos la conduit. Parallèlement, lui et sa femme ont récemment kidnappé un très jeune enfant, le bébé d'un de leurs voisins, qui est mort sans qu'ils le veuillent. Happé dans une dérive des sensations et de toute structure morale, comme siphonné, Marcos déambule dans Mexico au gré de ses courses de chauffeur. Conduisant sans ses lunettes, qu'il a perdues dans le métro, se masturbant chez lui l'après-midi devant un match de football à la télévision, tentant, sans succès, de faire l'amour à une collègue d'Anna au bordel, $\mathrm{y}$ parvenant avec sa femme, qui, comme lui, est tout à fait obèse, il subit de plein fouet les préparatifs fortement mystiques d'une procession populaire en l'honneur de la Vierge de Guadalupe, divinité tutélaire de tout le pays, et à laquelle son épouse est très sensible. Après s'être livré à une déambulation onirique dans les plateaux montagneux qui entourent la ville et avoir finalement pu coucher avec Anna, il décide de se constituer prisonnier et se rend une dernière fois chez cette dernière. Laquelle demande à son petit ami de les laisser seuls, et embrasse Marcos avant son départ pour la prison. Il quitte le coquet appartement, puis se fige dans le couloir, pissant dans son pantalon, revient sur ses pas, resonne à la porte. Et plutôt que de prendre le blouson qu'il avait oublié, sans dire un mot, il empoigne brusquement un large couteau de cuisine laissé là quelques instants plus tôt par le compagnon qui découpait une pastèque pour le petit déjeuner, et éventre la jeune femme, la laissant doucement se vider de son sang en la serrant contre lui. Puis il repart et quitte l'immeuble. Il progressera ensuite à genoux, torse nu, un sac en papier sur la tête, avec le reste des pénitents vers la cathédrale

20. Voir A. Ménil, «Image-mouvement, image-temps et plan ouvert», Cinémas. Revue d'études cinématographiques, $\mathrm{n}^{\circ}$ cité, p. I46-I79. 
de Mexico, siège des festivités en l'honneur de la Vierge. C'est là, hors champ, qu'il sera appréhendé par la police.

Selon Deleuze, le propre du cinéma moderne par rapport au classique - à savoir, d'avoir opéré un renversement des rapports entre mouvement et temps au profit de ce dernier ${ }^{21}$ - est qu'il ne fait plus du corps le simple instrument de la pensée, dont le siège avait toujours été, cinématographiquement parlant, le visage. Dans le cinéma moderne soutient Deleuze, le corps lui-même pense, en attitudes, en postures, en gestes. La pensée y a un corps. Mieux, elle est elle-même corporéité, libérée de ce que Deleuze, lecteur de Piaget, nomme le schème sensori-moteur : "C'est par le corps (et non plus par l'intermédiaire du corps) que le cinéma noue ses noces avec l'esprit, avec la pensée. $»^{22}$ Le corps, comme trace-palimpseste des sensations antérieures, est le présent de la coexistence de passés différents. Il n'est plus un simple outil ou instrument actualisant des idées ou valeurs qui sont autant de mots d'ordre abstraits venus d' "en haut», de la tête. Le corps pense et exprime sa pensée seul. Cependant, la façon dont il le fait est elle aussi particulière: elle ne consiste pas à agencer diverses positions de sujet et d'objet ou relations de cause à effet linéairement. Les implicites structures épistémologiques de ces couples binaires - tout comme leurs hiérarchies sous-jacentes - s'en trouvent défaites. Penser, connaître, savoir ne consistent plus à suivre un circuit clair et distinct, prédéterminé - et prédéterminant - entre cause et effet. Ce nouveau corps, cette corporéité coupée de la corporalité comme schème sensori-moteur, libérée du cerveau et de son joug sur les perceptions et leurs représentations, ayant subverti tous les circuits préformés, ne sait littéralement plus ce qu'elle sait, prise qu'elle est dans un nouveau type de savoir, inséparable du devenir qui la porte. Cette corporéité ne sait pas plus ce qu'elle veut ni ce qu'elle fait. Elle joint les causes à leurs effets de manière erratique et a-chronologique, par ruptures brusques ou souples, en fulgurant

2I. D. Zabunyan a bien montré comment, «[d]'un point de vue chronologique, toute interprétation expressément linéaire des deux volumes de Cinéma ruine la compréhension du geste philosophique deleuzien, et de la méthode qui lui est sous-jacente » (Gilles Deleuze. Voir, parler, penser au risque du cinéma, ouvr. cité, p. I43). Voir aussi A. Restivo, "Into the Breach. Between the Movement-Image and the Time-Image», The Brain is the Screen. Deleuze and the Philosophy of Film, G. Flaxman éd., Minneapolis, Minnesota University Press, 2000, p. I7I-I92.

22. G. Deleuze, L'Image-temps, Paris, Minuit, 1985 , p. 246; voir tout le fameux chapitre viII intitulé "Cinéma, corps et cerveau, pensée». Voir aussi S. Maeda, «Le corps en cinéma comme être positif selon Gilles Deleuze», Bigaku. The Japanese Society for Aesthetics, vol. 52, $\mathrm{n}^{\circ} 2$, automne 200I, p. 57-70. 
d'une strate ou d'un plateau du palimpseste des sensations à un autre. Ce qu'un tel cinéma des corps montre c'est alors, en définitive, comment les humains agissent en dysfonctionnant, ne fonctionnent que détraqués - comme les machines de Mille plateaux. Un paradoxe.

Dans une séquence tournée dans un couloir du métro de Mexico, Marcos et sa femme sont cadrés en plans rapprochés adossés contre un mur blanc. Elle vend à l'étal des colifichets, des bonbons, des gadgets, des réveille-matin. Leurs corps immobiles soutenant leurs faces impassibles, marmonnant entre elles, s'encastrent dans l'image très lentement, comme dans un ralenti infra-perceptible. Le son s'atténue, puis se coupe. La caméra zoome sur le visage de Marcos, son cou, ses joues, ses grosses lunettes carrées et grasses, ses poils, les pores de sa peau. Puis tourne sur ce qu'il regarde sans le voir: le mur d'en face, les passants, la chair des objets et des êtres peuplant son champ de perception. Ce plan-séquence produit l'étrange effet d'un plan qui serait en caméra subjective mais privé de la subjectivité dont il émanerait et vers laquelle il se recentrerait. Le visage, le regard, qui paraissaient en être la source, se massifient, deviennent chair. Un plan en caméra subjective semblant surgir d'un sujet sans corps et d'un corps sans sujet, filmé depuis un corps sans organes. Une confusion - de la sensation comme de l'entendement en résulte. Le regardcaméra, le plan se désincarnent. Les corps concrets de Marcos et de sa femme figés sur leur fond blanc se dépersonnalisent, deviennent abstraits, comme de la cire, comme leurs effigies dans un musée Grévin qui surgirait soudain d'on ne sait où, de l'image elle-même, entre nature morte et portrait de trois quarts. Indéterminés, mi-paysage, mi-objet (le mur), ils sont en même temps bien faits de chair. Le mouvement de caméra elliptique ne ramène rien à un axe d'origine autour duquel se structurerait le flux du regard par des positions d'objet ou de sujet. Les axes fuient au contraire, transversalement le long des murs blancs du couloir du métro. Tandis qu'au début du plan on peut penser que l'on voit le monde avec ou à partir des yeux de Marcos, la caméra vire à I 80 degrés et l'on découvre son regard pris en plein processus de chosification. La caméra tourne encore, ne nous laissant aucun répit malgré - ou peut-être grâce à - sa lenteur. Le regard-caméra est seul à l'ouvrage dans ce plan-séquence, autonomisé, et comme aspirant avec lui, dans sa nature machinique, la perception du spectateur. Le pour-autrui qui permet d'évider le pour-soi, pour parler comme Sartre, n'est pas l'autre sujet - ou l'image de son visage, son visage comme image - mais l'effet du travail de caméra. Non anthropocentrique plus encore que non humaniste, ce travail supprime tous les pôles de sub- 
jectivité - fût-ce comme «autres». Ce sont des choses qui chosifient le reste du monde, et elles-mêmes, via les mouvements de caméra. Mais les choses/corps bougent. Ce qui est vu en définitive, c'est alors l'inscience du sujet et la «connaissance» propre au corps qui se met en place, lente et physique, tandis que Marcos ne sait pas pourquoi il est là, où il est, pourquoi il y reste ni pourquoi il change de position, de côté, de forme, en partant, en bougeant. Il le fait pourtant, pour se déverser dans le flux des gens progressant plus ou moins vite dans le couloir, et qui l'emporte. «Il faut que la caméra invente les mouvements ou positions qui correspondent à la genèse des corps - écrit Deleuze -, et qui soient l'enchaînement formel de leurs postures primordiales. $\gg^{23}$ Le gestus de la station debout, originelle, constitue une telle réinvention, ici portée par les jeux du cadrage et du mouvement elliptique de la caméra. ${ }^{24}$

En plus de montrer ce fonctionnement paradoxal des corps, l'autre réussite de ce cinéma est d'en faire récit. Dès que le corps n'est plus un instrument implémentant une histoire dont le sens ne logerait que dans le cerveau - ce cerveau comme l'autre côté des yeux, comme le correspondant forcé du regard, la face interne du visage dont, selon Deleuze, le cinéma classique avait fait le siège de la pensée -, dès que le corps est force productrice plutôt que courroie de transmission au service d'un entendement purement rationnel, le récit lui-même se morcelle - ce qui ne veut pas dire qu'il devient forcément «abstrait», "abscons", "cérébral», «intellectuel». Il ne s'agit pas de retirer ou d'invalider toute jointure dans le corps de l'histoire, ou de le débarrasser de toute instance figurative - le cinéma des corps n'est pas un iconoclasme -, mais de les organiser différemment: selon les affects qui relient les corps et les histoires, plutôt que par des abstractions ou des taxonomies aristotéliciennes. Les affects et percepts sont, en définitive, ces liens paradoxaux évoqués plus haut, qui tissent la corporéité et le récit non téléologique l'un à l'autre. Qu'un programme d'une telle ambition, parfois radicale, ne produise pas un pur iconoclasme ou un pur formalisme, voilà la réussite.

La scène du meurtre d'Anna par Marcos est à cet égard exemplaire. Bien que l'interprétation puisse la rattacher au récit (par exemple: Marcos tue Anna parce qu'elle est le témoin, ou au contraire la cause de sa situation désespérée; ou parce que sa condition sociale et ce qu'elle en fait - se prostituer pour le plaisir tandis que tellement d'autres sont contraints

23. G. Deleuze, L'Image-temps, ouvr. cité, p. 262.

24. Sur le geste, voir M. Guérin, Philosophie du geste, Arles, Actes Sud, 1995. 
de le faire pour survivre - relèvent du scandale; ou encore parce qu'elle est le bouc émissaire, son meurtre le lavant de ses fautes à lui ${ }^{25}$ ), cette scène n'en constitue pas moins un déboîtage violent, résistant à tout agencement définitivement rationnel de telles explications, les mélangeant toutes si bien qu'elles finissent par constituer les différents strates ou aspects d'un affect général, indiscernables et indissociables des gestes et des postures, des craquements et des stases du corps qui le créent, le portent et le font passer dans le temps du récit. Tout télos est paralysé, comme ensablé dans l'ineptie apparente de la corporéité inerte. ${ }^{26}$ Dans le corps d'Anna, dans les configurations politico-sociales qu'elle agence avec, quelque chose d'intolérable, d'insupportable a été saisi par Marcos: dans les termes de Deleuze, "quelque chose de trop puissant, ou de trop injuste, mais parfois aussi de trop beau, et qui dès lors excède nos capacités sensori-motrices ». ${ }^{27}$ Le corps normé a craqué (Marcos se pisse dessus), mais il se refait immédiatement à nouveaux frais, monstrueusement (il sonne, il empoigne, il éventre, sans une once de pitié, tout en berçant tendrement dans ses bras ensanglantés le quasi-cadavre pour ses derniers instants): en lui s'est morcelé l'ordre des significations au profit d'un autre, à tous égards hors la loi. Le corps comme lieu de passage des affects, de leurs connexions, de leurs discours. Le corps comme transistor: malléable, ductile, amoral, et à travers lequel circulent toutes sortes d'affects. Cadré plein pied totalement immobile dans le couloir, dans une mate lumière bleu ciel, le seul mouvement est l'ombre de la tache d'urine qui grandit à vue d'œil sur son pantalon, descend légèrement sur l'une des jambes, et la petite flaque qui se forme près des chaussures. Visuellement, c'est comme la fissure intime - liquide, humectante, et enfantine d'un bloc de marbre qui se propage sous nos yeux. Rarement une image si cadrée plein centre et si fixe aura montré, comme en direct (pisser sur commande valant bien, du point de vue du jeu, les exploits lacrimaux, souvent présentés comme l'aune d'une performance d'acteur), le bascu-

25. Les analyses de R. Girard seraient à cet égard de toute utilité ici, comme le serait un examen en termes de gender studies. Dans le dernier essai du présent volume, M. Millan identifie pour sa part les corps politiques mis en place par Bataille dans le ciel d'après les études postcoloniales.

26. Du point de vue du spectateur, selon P. Dubois, «nous ne nous souvenons donc pas (bien), malgré ce plaisir, du pourquoi et du comment de l'histoire, mais notre conscience perceptive (pour ne pas parler de notre inconscient perceptif) et surtout notre mémoire, notre mémoire vive, retient d'abord des images, ou plutôt des "figures" justement» ( «a question du figural ", art. cité, p. 55).

27. G. Deleuze, L'Image-temps, ouvr. cité, p. 29. 
lement du corps dans ce qui (lui) échappe et (l')emporte, la violence d'un tel basculement, la force de sa dynamique.

\section{Teenagers in fabula}

Elephant de Gus Van Sant est quant à lui directement inspiré du massacre de douze adolescents et d'un adulte au lycée de Columbine dans le Colorado, en 1999, par deux jeunes hommes équipés d'armes de guerre (et qui finirent eux-mêmes par se donner la mort). Le film montre par interstices les faits et gestes d'une demi-douzaine d'adolescents dans leur environnement quotidien - le lycée - jusqu'à construire une mosaïque de portraits propre à faire ressortir la décompensation des deux jeunes meurtriers, mais aussi - surtout? - à montrer les adolescents à l'œuvre, c'est-à-dire leurs corps au quotidien. Premières expériences d'acteurs, dialogues semi-improvisés, plans en steadycam (à plus de 70\%) et en longs travellings: Elephant observe les teenagers avec souplesse mais assiduité: par-devant, par-derrière, de côté, en plongée. Ces derniers déambulent doucement sur le circuit que semble constituer le lycée: longs couloirs, vastes pelouses, spacieuse bibliothèque, gigantesque cafétéria, larges corridors et halls d'entrée, comme dans une boîte à chaussures macroscopique, l'expérience consistant à faire circuler puis à scruter la lente dérive des corps fermes, propres, gras, ou encore anorexiques des teenagers. ${ }^{28}$ Le film ne montre aucun cours, comme pour escamoter la fonctionnalité officielle de l'endroit, le transformant ainsi en la sorte de non-lieu dont parle l'anthropologue Marc Augéers, un no man's land ou lieu sans qualités, propre à l'«entre ", à ce qui, en un surplace paradoxal, glisse hors du

28. Voir C. Boudet-Lefort, "Des labyrinthes où s'égare le corps», Le Corps filmé, A. Grunert éd., Paris, CinémAction-Corlet, 2006, p. 8I-87. Dans une lecture surdéterminée par le massacre final au point d'en devenir quasi lazaréenne, et confondant la structure narrative éclatée du récit avec une nécessité téléologique, F. Bernard de Courville voit dans les corps d'Elephant des fantômes en sursis, clos sur eux-mêmes, voués à la mort, manquant en cela leur intrinsèque et vitale plasticité: "La pesanteur et la grâce", Gus Van Sant Indé-tendance, Y. Calvet et J. Lauté éd., nº 4I d'Éclipse, 2007, p. I28-I37. En un étrange raccourci Bernard de Courville écrit: "[Van Sant] redéfinit le cinéma : faire du cinéma, c'est montrer des corps déjà morts.» De façon générale, elle étudie la dialectique des corps d'Elephant en termes de verticalité - les disposant entre terre et ciel, légèreté et pesanteur. Je propose au contraire de les considérer dans une horizontalité permettant d'interroger leur malléabilité et leur plasticité.

29. M. Augé, Non-lieux. Introduction à une anthropologie de la surmodernité, Paris, Seuil, 1992. Voir aussi Les Cahiers du cinéma, $\mathrm{n}^{\circ} 583$, octobre 2003 et le dossier consacré à Elephant. 
monde des fins pour simplement être là. Filmé comme délesté de son rôle d'enseignement, le lycée se livre tout entier à celui de théâtre de socialisation, mais à un rythme ralenti. Espace-temps idoine pour ce qui s'y joue au niveau des corps. ${ }^{30}$ Que ce soit dans l'ombre (en fond de couloirs) ou dans la luminosité naturelle (à l'avant-plan, là où les portes vitrées du lycéeboîte entrent en contact avec le dehors), la caméra de Van Sant pénètre le corps du bâtiment orthonormé pour y mettre en valeur les mouvements des jeunes gens circulant avec plus ou moins d'énergie, de volonté, de nonchalance. Archétype d'un dispositif de mise en scène très volontariste pour ce qui est de l'environnement physique, le film colle pourtant pardessus ce décor fixe de décalcomanies la pure et calme fluidité des corps, encore augmentée par la construction non-chronologique du récit et la réitération de plusieurs scènes (donc de lieux) filmées de points de vue différents. On se demande souvent si les adolescents ne sont pas en train de glisser sur des patins en feutre et si le sol n'est pas un parquet vitrifié. Là-dessus, vingt pages de script tout au plus; un synopsis d'à peine deux pages consistant en fait en une liste de titres de scènes; et un réalisateur, Gus Van Sant, et son producteur, Dany Wolf, qui ont dessiné un plan de l'école vue de haut et y ont inscrit en différentes couleurs les trajets des protagonistes, avec les points d'intersection et les diférentes sources sonores disposées hors-champ..$^{3 \mathrm{I}}$ En définitive s'impose la forte impression que le mouvement souple des corps à travers la boîte est le motif central du film, son obsession même, sa raison d'être. Travellings latéraux d'un corps déambulant, puis d'un autre, dans une autre direction, après jonction, et ainsi de suite, sans jugement, sans ordonnancement moral, tout en observation, en fascination retenue pour ces corps désinvoltes ou tendus - retenue justement par les bords du dispositif physique de la mise en scène: les murs de la boîte-lycée jouant le rôle de contenant spéculaire.

Le corps adolescent : comme Larry Clark, Gus Van Sant parvient à en

30. Dans son remarquable ouvrage E. Arnoldy saisit ce nœud à travers l'œuvre de Van Sant: «Dans le même élan, depuis Mala Noche et jusqu'à Gerry, Elephant ou Paranoid Park, une des singularités de ses films est en effet d'opérer un grand écart incessant entre deux limites extrêmes du cinéma: filmer les corps, filmer les paysages ou comment filmer des corps dans un espace", Gus Van Sant. Le cinéma entre les nuages, Crisnée (Belgique), Yellow now, 2009 , p. 98.

3I. C. Béghin a étudié la thématique comme la stylistique d'Elephant à travers son usage de la dimension sonore articulée autour d'un double modèle: celui, étal, du dehors (ciel, bruits de gare, d'aéroports, de forêts), figurant un «monde qui file vers des lointains", et celui, elliptique, formé par les «couloirs, circulations, croisements et structures» du lycée, «Cut-up en plein ciel», Vertigo, n² 27, mars 2005, p. 23-26. 
montrer l'essentielle polymorphie ou plasticité dans Elephant, mais il le fait selon des régimes bien à lui. C'est d'une part, la langueur particulière, faite à la fois de mollesse et de fermeté - dans la démarche, le port de tête, certains gestes ou encore l'intonation et le rythme de la voix, chez presque tous les garçons du film, y compris chez les deux jeunes tueurs, ce qui ne laisse pas d'introduire un certain malaise. ${ }^{32}$ Mais c'est aussi la raideur ou la crispation qui structurent les corps d'autant mieux qu'elles sont internes (par exemple dans l'acte de régurgitation volontaire, devenu routinier, voire naturel, pour les trois jeunes filles anorexiques qui se font vomir dans les toilettes tout juste après avoir déjeuné à la cafétéria). Et c'est encore la gêne rentrée qui replie les chairs sur elles-mêmes, comme une invagination de tout le corps (chez la jeune lycéenne bibliothécaire complexée, forcée de se changer pour le cours de sport en s'enfonçant carrément à l'intérieur de son placard pour y disparaitre de la vue des autres filles). Ces trois régimes de plasticité corporelle sont mis en rapport entre eux plutôt que posés en catégories fixes et absolues: lorsque les filles reluquent les garçons, lorsqu'elles ricanent juste derrière celle qui se change dans son armoire, lorsque les garçons se rencontrent et se prennent en photographie, c'est une économie figurale qui entre en contact avec une autre comme à une autre modalité - déniée, escamotée, impliquée - d'elle-même.

Dans un plan-séquence long de trois minutes alternant travellings avant et latéraux, Elias, un lycéen épris de photographie, rentre dans l'école par la porte principale puis progresse dans un long couloir plutôt sombre vers le laboratoire de photographie. Ce couloir est bordé par le vestibule d'entrée et, au loin, par des portes-fenêtres diffusant faiblement une luminosité opaque, celle du jour. Entre ces deux extrémités le corridor comporte de nombreuses intersections, ainsi qu'une large baie vitrée orthonormée et latérale, laissant cette fois entrer la vive clarté extérieure. De sorte que, longitudinalement comme latéralement, ce couloir est fait de cadres dans le cadre - qu'il constitue lui-même, puisqu'on en perçoit les angles droits du plafond à l'image. Le spectateur voit ainsi un cadre fixe, celui de la coupe transversale du couloir à travers lequel Elias avance, mais dans lequel flottent les cadres de la porte du fond. Ce dispositif de

32. Notamment dans la scène, terrible de concision, où le jeune champion de football et son amie possiblement enceinte, qui se sont réfugiés dans la chambre froide de la cafétéria où pendent des quartiers de viande, sont abattus à bout portant par l'un des tueurs. La détonation comme son résultat restent hors champ, seule la métonymie avec la chair congelée et sans vie de la viande de boucherie dit l'acte, en un raccourci glaçant. 
surcadrage ${ }^{33}$ se dédouble exactement, cette fois en forme rectangulaire, lorsque la caméra pivote à 90 degrés et filme le profil d'Elias avançant, et non plus son dos. Cadres gigognes, cadres mouvants, cadre fixe (l'écran), méta-cadre ou agencement de cadres dans le mouvement: «dedans» tous ces cadres - mais est-ce bien de cette notion qu'il s'agit toujours ? ${ }^{34}-$, un corps, celui d'Elias, des corps, ceux de tous les autres lycéens qu'il croise. Certains se figent parfois, d'autres non et demeurent en mouvement. Plusieurs apparaissent comme ça dans l'image, du fait des différentes vitesses de pas, puis disparaissent. Si le spectateur perçoit le mouvement général des corps marchant, il ne peut le rationaliser en un système réglé ou une structure ordonnée. Grâce à des effets de ralenti à la lisière du perceptible, Elias semble par moments un peu grisé, en état d'ébriété, comme riant intérieurement. La musique électronique, douce mais répétitive, ajoute à cette sensation de glissade perceptive. Que fait Elias? Il marche, progresse, avance le long d'un sentier. Dans cette séquence aucune notion de picturalité propre au cadrage classique ne se détache. L'on ne perçoit pas davantage le cadre comme un cache camouflant un mouvement ayant lieu dans la matière sombre de ce qui est rejeté par la lumière de l'écran hors d'ellemême, comme dans la théorie de Bazin. Alors que perçoit-on? Rien de moins que le cadre comme pur mouvement, le cadre comme infini cadrage à l'origine de cette corporéité à l'œuvre, en pliures, en déplacement. Il ne s'agit plus de corps dont on saisirait le mouvement par contraste avec un arrière-plan sur lequel se figurerait la progression, c'est le cadrage luimême qui montre la plasticité intrinsèque de ces corps qui tout à la fois traversent et peuplent le cadre. Comme si l'acte de peupler était à rapporter à une porosité ou une précarité essentielle; et comme si, corrélativement, l'acte de passer ou de traverser était fait de micro-séjours ralentis et bien réels. Cette mobilité paradoxale de la chair, cette motilité, n'est pas filmée directement, n'est pas saisie par ou dans le cadrage, si sophistiqué soit-il; elle n'en émane pas non plus toute faite comme d'un moule; elle y est produite, elle y est agencée. Le cadrage n'est pas un écrin, c'est un crible, un filtre, ou une machine de capture abstraite, une production virtuelle. La corporéité y est créée, elle ne traverse rien. Plus précisément,

33. Voir J. Aumont, L'Image, ouvr. cité, p. II6.

34. Pour reprendre la typologie développée par J. Aumont, il semble qu'Elephant pratique simultanément un surcadrage et un refus du centrement, comme s'il s'agissait de surcadrer les corps pour mieux les faire fuir, pour montrer directement leur fuite plutôt que la filmer dialectiquement (ibid., p. II8). 
elle est créée par la dialectique que l'acte de cadrer agence avec le horscadre chaotique, comme si des instances ou occurrences de la corporéité première, le chaosmos, s'en échappaient brusquement ou étaient happées par la lumière comme des insectes pour passer dans le cadre (c'està-dire l'écran), et y devenir des taches de chair, formant avec d'autres des archipels en perpétuelle reconfiguration. L'effet de ce type de cadrage comme ontogenèse d'une corporéité générique est à son comble lorsque Elias marche devant une énorme peinture murale représentant, légèrement plus grands que nature, des adolescents de toutes races: la surface des corps peints n'entre pas en conflit ni ne fait la compétition avec l'image cinématographique du propre corps d'Elias. Bien au contraire, elles se mélangent: dans l'allure et la chaleur du mouvement de marche le corps d'Elias est le bienvenu parmi l'énorme cartouche de teenagers peint contre le mur. L'image du profil de son corps est avalée, devient soudain surface, mais alors ce sont les rigides corps peints qui semblent se mouvoir aussi. ${ }^{35}$ L'incessante progression le long du couloir-cartouche doublement cadré (physiquement et cinématographiquement, à la façon d'un méta-cadre ou cadre au carré) devient une métaphore des 24 images par seconde définissant le cinéma, mais aussi, généalogiquement, des flip books de la fin du XIX ${ }^{\mathrm{e}}$ siècle, ces ancêtres d'un certain cinéma classique, petites liasses rassemblant une multitude de postures (le plus souvent un homme ou un cheval avançant), une par feuille, et dont il s'agissait d'animer le mouvement théorique, ou encore potentiel, par un brusque frottement du pouce contre la tranche, faisant ainsi défiler les feuilles-images et laissant voir l'effet de leur mise en série. ${ }^{36}$

Mais un autre passage de la séquence s'impose au commentaire, long de trois ou quatre secondes tout au plus: à l'avant-plan le profil d'Elias avance quand, tout à coup, comme naissant de sa pomme d'Adam depuis l'arrière-plan - mais ces notions d'avant- et d'arrière-plan, de palimpseste

35. A. Tylski y voit une allusion à la portraiture égyptienne antique afin de pouvoir insister sur le thème funéraire, "Gus Van Sant et le Minotaure», Cadrage, [en ligne], [URL: http://www.cadrage.net/films/elephant/elephant.html], consulté le 26 février 20 Io. Bien que ce sujet - la mort - soit structurant à travers le film, je propose de ne pas le surinterpréter (de le lire ou le voir à chaque image ou presque) mais plutôt de déceler les séries élémentaires - et parmi elles, la Vie comme jeu de l'informe - qui se mettent dialectiquement en place.

36. S. Charlin a étudié cette esthétique du bord à propos du cadre photographique: «Autour du cadre: la zone de l'image", Effets de cadre. De la limite en art, M.-C. Ropars éd., Saint-Denis, Presses universitaires de Vincennes, 2003, p. 2I-27; voir aussi G. Lambert, "Cinema and the outside», The Brain is the Screen, ouvr. cité, p. 253-292. 
iconographique, ont-elles encore un sens dans un cinéma si prompt à travailler la surfacialité de la chair et sa puissance d'autodifférenciation, de simultanéité ? - le visage de profil d'une fille surgit, rebondissant gentiment dans l'image, apparaissant, disparaissant, changeant de forme, devenant paysage, rappelant l'«espace pré-hodologique» dont parle Deleuze : «comme une fluctuatio animi qui ne renvoie pas à une indécision de l'esprit, mais à une indécidabilité du corps $» .^{37}$

Il est significatif que cet hypnotique plan-séquence ${ }^{38}$ montre, à un niveau diégétique, Elias en chemin vers le laboratoire de photographie du lycée. Tout du long il semble pris dans la ligne de fuite du couloir, dont l'extrémité (l'ouverture?) sont les portes avec leur baie vitrée à la luminosité opalescente et floue, comme pour marquer l'ailleurs absolu de l'indétermination corporelle et existentielle. Comme si le but de sa déambulation était ce dehors abstrait, ou plutôt, qu'un tel dehors, une telle extériorité à la corporalité (le chaos du cadre dynamique dont parle Deleuze) fasse pression sur le dedans. Cependant, le dehors demeure constamment vague, brouillé, de la même manière que la musique semble maintenir une note abstraite, plus ou moins audible, vibrant doucement, comme neutre, pendant tout le plan-séquence. Enfin, il est aussi significatif qu'Elias finisse par dévier de sa trajectoire rectiligne quelques mètres avant d'atteindre la porte à la fin du couloir, et oblique vers la droite en une légère ellipse pour pénétrer dans le laboratoire rempli de négatifs photographiques déroulés et pendant du plafond en une série de verticales bien visibles, comme pour réitérer, cette fois de la façon la plus matérialiste et métonymique qui soit, ce qui vient juste d'arriver aux corps dans le couloir: leur devenir-image. Les mouvements que le couloir suscite, ce qui survient aux corps s'y engageant et filmés par Gus Van Sant devient comme une méta-camera oscura. 39

Ce n'est pas que les corps soient extensibles à l'infini en une dialectique être/non-être rationalisée, ou vu/pas-vu, comme à cache-cache ou à colin-maillard («maintenant tu me vois, maintenant tu ne me vois

37. G. Deleuze, L'Image-temps, ouvr. cité, p. 264.

38. T. Clolus a étudié cette notion autour de la représentation du corps dans Gerry (2002): "Gerry, ou le corps deleuzien de Gus Van Sant», Fin de séance, [en ligne], [URL: http:// www.findeseance.com/Gerry-ou-le-corps-Deleuzien-de-Gus], consulté le 26 février 2010.

39. À un niveau thématique, S. Bouquet et J.-M. Lalanne voient dans ce laboratoire une «utopie» ou l' «espérance d'un bonheur» auxquelles il est mis fin par les meurtres: Gus Van Sant, Paris, Cahiers du cinéma, 2009 , p. I57. 
pas»). Ici, c'est le cadre qui est la frontière ouvrant au poreux de l'être, mettant toute frontière dans les corps plutôt qu'entre eux, y disposant le cadrage lui-même. Selon Deleuze, l'histoire de l'art occidental est avant tout celle de la composition. Composer avec, et directement au sein de la multiplicité de l'être. L'art, selon lui, ne fait que cela, pure anthropologie en acte et sans frontière, immanente, généralisée, et paradoxale. Cette multiplicité fondamentale est sans cesse décomposée et recomposée par une force qui s'appelle la Vie, et l'art (ici cinématographique) consiste à la percevoir, la capturer, la manifester en se laissant prendre par elle. Et une fois figurée en une stase paradoxale (l'intime mouvement générique en toute forme), à la renvoyer aussi énergiquement que possible vers le chaos via les œuvres. Travail ardu, à n'en pas douter. Question de dosage et de résistance aux élans de toutes sortes - vers la représentation molaire comme vers l'autisme de la décomposition moléculaire. Dans les mots de Dominique Villain :

Cette capture de forces semble pouvoir également définir ce qui se passe au cinéma, dans la prise de vue. Les éléments que le cadrage cinéma compose, décompose et recompose sont par exemple comme en peinture : la lumière, la couleur, les différentes places des différentes figures (dans le champ), leur proximité, présence, netteté, ou leur éloignement, leur flou, le jeu des avant-plans et des arrière-plans (dans le plan), des fonds (le décor) et de la surface (l'écran), des points de fuite et des points de vue, des directions de regard, du rapport au spectateur, etc. ${ }^{40}$

Cadrer, c'est avoir un oil dans la caméra et l'autre dehors; c'est être à la fois myope et précis des yeux. Cette position incongrue offre parfois la possibilité d' «inventer une ubiquité» (ibid., p. I6) ou un certain type de voyance qui rend disponible à ce qui prend - ou perd - corps autour, dehors, ailleurs. Quand le cadre court après le corps et le manque, ne l'attrape jamais complètement comme dans Elephant, cela raconte quelque chose: un récit sans télos, une histoire sans organes, une histoire dont nous ignorons la forme - et partant, le sens -, de la connaissance de laquelle nous demeurons physiquement et perceptivement dehors ou extérieurs. Un sens désincarné, à la limite de l'abstraction pure. Le seul avec et à travers lequel nous puissions, paradoxalement, vivre. Il ne s'agit bien sûr pas d'un simple non-sens ou insensé, mais d'une façon de permettre au sens de n'être pas prisonnier de lui-même, de n'apparaître que lorsqu'il s'en libère. Un corps qui fuit à travers une histoire non téléologique, filmé de

40. D. Villain, Le Cadrage au cinéma, ouvr. cité, p. I23. 
façon à produire non pas une image de lui mais à être annexé au corps de l'image, et, ce faisant, à révéler les puissances de celle-ci, accomplit cela.

La tension, immémoriale, entre la force et la forme, entre la philosophie stoïcienne et le platonisme, entre le matérialisme empiriste et l'idéalisme sous tous ses avatars, entre le monisme et les dualismes - en un mot: entre la pure immanence chère à Deleuze et la transcendance - ne s'arrête jamais. Tout le génie du cinéma consiste à manifester, en l'agençant dans ses dispositifs, ce qui résiste et échappe à la fixation ultime, cadavérique, et à en potentialiser la bonne nouvelle - la vitalité - pour nous autres, les vivants. Ces deux films, qui pourtant montrent meurtre après meurtre, chambre froide et lieux du crime, y parviennent avec éclat. 


\section{Politiques}





\section{:::}

\section{Insurrections de l'amour en contexte matérialiste: Garrel, Costa, Waked}

Adoration, sentimentalité, passion, amour... une terminologie aux origines et aux usages théologiques qui en disqualifient l'utilisation dans le cadre de la pensée critique. Noyés dans les «eaux glacées du calcul égoïste»; débusqués par Freud et par Lacan; remplacés philosophiquement par le vocabulaire du désir, du principe de plaisir ou de la joie spinoziste ; congédiés par les artistes qui cherchent à remonter plus haut, aux sources organiques des pulsions; défigurés, vidés et confisqués par le monde de la communication: les termes par lesquels le sujet pourrait indiquer ses affects demandent réinvestissement avant de retrouver une légitimité dans le champ critique. Nous allons observer comment certains cinéastes matérialistes et formalistes œuvrent à réinvestir le terrain de l'amour, un amour insubordonné, insurrectionnel, qui refuse autant les dévoiements et les régressions que l'abandon des territoires du sentiment et de la passion. En voici trois: Philippe Garrel, à l'échelle d'une ouvre, consacre une idole et invente une adoration moderne et syncrétique; Pedro Costa, décrivant Jean-Marie Straub et Danièle Huillet en travailleurs de l'image, libère le respect de tout principe d'assujettissement; inversant l'image de corps humiliés, Sharif Waked transforme la colère en puissance d'affirmation.

Dans sa dimension anthropologique, le cinéma tout entier est une vaste enquête formelle sur la présence. Simultanément trace, reconstitution et clignotement, le matériau figuratif s'y manifeste à l'état de fétiche, il est un prélèvement, offrant ou non hypothèse sur l'être. Forts d'un tel préalable, les cinéastes ici observés élaborent des économies figuratives qui déploient ces données formelles pour les mettre au service du réinvestissement des passions. 


\section{Élaboration d'une idole. Nico par Philippe Garrel}

Entre 1978 et 1979, entre Le Bleu des origines (1978) et L'Enfant secret (I979), s'achève le moment le plus expérimental du cinéma narratif français. C'est la fin des deux décennies les plus brillantes et audacieuses dans l'histoire du cinéma en France (1957-1978 ${ }^{\mathrm{r}}$ ), qui a vu s'épanouir collectivement les formes radicales et les gestes artistiques transgressifs, c'est-à-dire, entre autres, dans le désordre et pour s'en tenir au terrain narratif, les œuvres de Jean Rouch, Jean-Luc Godard, Jacques Rivette, Jean-Marie Straub et Danièle Huillet, Jacques Rozier, Sylvina Boissonnas, Yvan Lagrange, Jean Eustache, Christian Boltanski, Marcel Hanoun, Pierre Clémenti, Patrick Deval, Jackie Raynal, Daniel Pommereulle, Jean-Pierre Kalfon, Marc'O, Jean-Daniel Pollet, Lionel Soukaz, Diourka Medveczky, Adolfo Arrieta, Marguerite Duras, Serge Bard, Luc Moullet, Guy Gilles, Gérard Courant, Philippe Vallois ou le radical et encore trop méconnu Jean-Pierre Lajournade, dont Philippe Garrel, qui lui doit beaucoup, fut l'assistant.

Au sein de cette brillante constellation, l'œuvre de Philippe Garrel frappe par sa constance en matière d'invention formelle et d'iconographie. Depuis 1964, elle déploie les mêmes fables de solitude à deux permettant d'ouvrir un immense éventail de plans-séquences en travelling dans les jardins, les carrières ou les déserts, pour traiter du même héroïsme romantique désespéré, en une quête permanente qui change souvent de site mais jamais de sens. La constance formelle se systématise à partir de 1970: sept films avec Nico, comme autant de panneaux de la même fresque continuée, tantôt muette, tantôt sonore, tantôt en noir et blanc, tantôt en couleurs, mais toujours peinte sur le même mur affectif, un heptatyque de style gothique psychédélique. Chacun des films de la fresque se consacre à célébrer Nico et constitue ainsi l'un des plus longs poèmes d'amour et de vénération que l'histoire de l'art nous ait légué. «Filmer, comme je l'ai fait pendant dix ans, une femme que l'on aime est une chose en soi assez folle... $\gg^{2}$ La disparition de l'objet d'amour va provoquer un tournant formel.

I. Sur la date de 1957 , voir « "For its the critical faculty who invents fresh forms". History of the forms, 1960-2000", The French Cinema Book, M. Temple et M. Witt éd., Londres, British Film Institute, 2004, p. 230.

2. P. Garrel, T. Lescure, Une caméra à la place du caur, Aix-en-Provence, Bureau éditorial Admiranda - Institut de l'image, I992, p. 89. 


\section{Les idoles païennes}

La première période de l'œuvre de Garrel mène des Enfants désaccordés (1964) au Lit de la Vierge (1969). Inspiré par Gérard de Nerval, Arthur Rimbaud, William Blake, Sigmund Freud et sans doute Che Guevara, Garrel s'efforce de porter le cinéma à l'intensité des grands mythes romantiques, un cinéma dont le génie descriptif s'emploierait sur un mode non pas réaliste mais visionnaire. Garrel invente un cinéma de voyant, c'està-dire d'une vision intensive du réel, capable d'en dénuder les affects et les pulsions fondamentales. Pour ce faire, les films recourent à une figuration tantôt mystique (Marie pour mémoire, 1967; Le Lit de la Vierge), tantôt freudienne (Le Révélateur et La Concentration tournés en I968), qui dans les deux cas ramènent à une pathologie familiale. Un immense fantasme d'abandon se manifeste en fables structurées par blocs épisodiques - souvent, un épisode ou une saynète équivalant à un plan-séquence, en une extension maximale du principe des films Lumière - où les travellings complexes inspirés de Max Ophuls, Orson Welles et Jean-Pierre Lajournade annulent le temps et transforment les espaces concrets en déserts psychiques.

Parallèlement, Garrel intervient dans le champ de la pop culture, qui transforme les rituels d'adoration autrefois d'ordre mystique en cérémonies commerciales et fabrique donc en masse des idoles païennes prêtes à l'emploi. Sous réserve de recherches ultérieures, Garrel réalise au moins six sujets pour l'ORTF en 1967. Ce pan méconnu de son travail se répartit en quatre types: sujet de société; reportage musical; mise en scène de chansons; présentations des sujets par Garrel lui-même. Quels que soient leur longueur ou leur genre, les sujets traités se rapportent à la jeunesse et à la musique, phénomènes emblématiques de ces années pop dont témoignent notamment Les Idoles de Marin Karmitz (1964, 7 minutes, sujet pour l'ORTF), Masculin féminin de Jean-Luc Godard (1966), Les Idoles de Marc'O (1968). Ils sont réalisés pour les émissions «Bouton rouge » et «Seize millions de jeunes » produites par André Harris et Alain de Sédouy. ${ }^{3}$

3. " "Seize millions de jeunes", A. Harris et A. de Sédouy, deuxième chaîne, I8 avril I964 8 mai 1968 (II7 numéros), et "Bouton rouge", deuxième chaîne, I6 avril I967 - II mai I968 (26 numéros)» (M.-F. Lévy, «La jeunesse irrégulière dans la télévision française des années soixante: une absence troublante", Revue de l'enfance "irrégulière", ${ }^{\circ}$ 4, 2002, [en ligne], [URL: http://rhei.revues.org/index53.html], consulté le 27 février 20IO). A. Harris et A. de Sédouy sont les futurs auteurs du célèbre Français si vous saviez (1973) 
«Bouton rouge», coproduite par Michel Taittinger et Jean-Pierre Fraimbois, introduit la culture rock and roll à la télévision publique française, les stars anglo-saxonnes y règnent: Jimi Hendrix, Eric Clapton, Pink Floyd... Il s'agit principalement d'accompagner la propagation des modes de pensée, de comportement et de consommation américains (au sens restreint de l'Amérique WASP récupérant la musique des Afro-Américains alors en lutte). La télévision et la presse s'efforcent de transformer la génération du baby-boom en "public», terme qui devient peu à peu une position politique résolument passive dont le spectacle fournit le modèle, et qui refoule la conscience collective de soi comme appartenant à un peuple. Dans un tel contexte frappe la radicalité politique de Garrel qui injecte de la critique partout où c'est possible, à commencer par la diversité inventive du traitement des sujets abordés, dans des formes et des longueurs encore non standardisées.

Pour introduire Les Jeunes et l'argent ( «Bouton rouge / Seize millions de jeunes», I8 juin 1967), sur fond sombre et neutre, Garrel annonce calmement :

Bonjour, je dois vous présenter notre émission Les Jeunes et l'argent. Elle se propose de montrer l'attitude des jeunes gens qui s'adaptent au système capitaliste, entendez par là ceux qui ont déjà accepté de lutter pour l'argent et plus précisément ceux qui ont réussi. Cette soirée étant placée sous le signe de la jeunesse, c'est à eux que je m'adresse, parce que moi je commence vraiment sérieusement à manquer d'air.

Pour Ronnie et les mots (7 minutes, «Bouton rouge», 2I mai I967), le chanteur français Ronnie Bird et une comédienne non identifiée dont le personnage se prénomme Marielle, transpositions manifestes du couple Jean-Pierre Léaud et Chantal Goya de Masculin féminin, transforment une chanson en sketch sur le langage des jeunes gens. Dans un décor abstrait composé d'un canapé, d'un téléphone et d'un mur sur lequel il est inscrit: "Avant d'apprendre à vivre il faudrait apprendre à parler», les deux acteurs et le réalisateur se livrent à une démonstration de force amusée en faveur du vocabulaire à la mode, comme Jean Eustache pourra le faire plus tard dans La Maman et la Putain (1973). À la manière d'une langue étrangère, l'idiolecte des jeunes est sous-titré : «C'est la gerbe, c'est l'angoisse, c'est vraiment l'angoisse» se voit commenté : «adjectif péjora-

consacré à la Résistance, et bientôt producteurs du Chagrin et la pitié (1969), consacré à la collaboration - parmi les autres réalisateurs de «Bouton rouge», on compte d'ailleurs aussi M. Ophuls. 
tif», tandis que «Qu'est-ce qu'on glande?» est traduit: «Que pourrait-on faire?» La défense et illustration des marqueurs langagiers de la jeunesse alimente un dandysme qui va s'exercer aux dépens du public. Le téléphone sonne, Marielle transmet: "C'est un type qui regarde l'émission, il dit qu'on n'est pas du tout représentatifs de la jeunesse actuelle, qu'on est des petits cons, qu'est-ce que je réponds?» Reprenant des mimiques de Léaud, Ronnie désarçonné regarde la caméra et demande: "Qu'estce qu'on fait, on arrête? »; la voix off de Garrel répond : "Non, non, on continue, répondez ce que vous voulez.» Marielle raccroche alors sur ces mots peu fréquents à la télévision française de l'époque (deux chaînes gouvernementales), surtout dans la bouche d'une fraîche jeune fille qui s'adresse à son audience: "Ta gueule.» Source majeure pour l'iconographie Mods ( «je suis passé au Drug ${ }^{4}$, j'ai acheté Melody Maker»), Ronnie et les mots décline les façons d'utiliser une chanson: chantée; dite ou lue sans musique; récitée comme une rêverie... Un gros plan des deux comédiens face à la caméra, avec en off la voix de Ronnie, «je dis ça mais je pourrais dire autre chose", constitue l'articulation directe qui mène du réalisme abstrait de Masculin féminin à l'abstraction sensuelle du Révélateur.

Handa et la sophistication (II minutes, «Bouton rouge », I8 juin I967), sans doute le film le plus marquant de la série, dresse le portrait d'un type sociologique émergent à l'époque, le dandy féminin (figure inenvisageable selon la charte masculine du dandysme baudelairien). Filmée en très gros plans et au moyen d'iris, Handa décrit ses passionnantes activités de consommatrice avertie: choisir des vêtements, regarder des bijoux, boire du thé à la mosquée de Paris, essayer une pipe à opium... Elle fut le modèle d'une bande dessinée psychédélique, La Saga de Xam, scénarisée par Jean Rollin et dessinée par Nicolas Devil, parue chez Éric Losfeld la même année. Comme ses vêtements, le monologue hautain et allumé de Handa est tissé de perles inoubliables adressées directement à la caméra avec la voix la plus snob et traînante possible: "Moi je suis totalement pour la décadence»; «Les voisins vivent à l'envers, ils courent à 8 heures du matin et nous réveillent, c'est pas bien »; "J'adore toucher les serpents, c'est très beau, très fantastique »; "Je suis assise devant un portrait de moi, un psychédélique portrait»; "Je parle, je parle mais... il est l'heure d'aller toucher quelques dentelles»; «De toutes façons vous aurez beau faire un effort, vous aurez toujours l'air de sortir de chez Bodygraph » (c'est-à-dire

4. Drugstore des Champs-Élysées. 
vous serez toujours mal habillé). Ces dix minutes parfois hilarantes, parfois embarrassantes (par manière de bravade contre la routine, Handa qui finit sa nuit se promène dans le métro du petit matin parmi les travailleurs immigrés effondrés de fatigue) se terminent sur une ultime provocation, filmée en zoom avant, les yeux droits dans l'objectif: «Bon, si vous êtes tombés amoureux de moi, vous pouvez m'écrire. Vous ne risquez rien, vous n'avez aucune chance, j'ai horreur, mais vraiment horreur, des gens qui regardent la télévision.»

À la fin des rushes de «Bouton rouge» (émission du I8 juin 1967), deux prises avec clap, de respectivement quarante et trente secondes, nous montrent Garrel en légère contre-plongée sur fond de rampe de studio. Tout jeune (il a dix-neuf ans), très maigre et pâle, il déclare de sa voix douce :

Voilà, c'était «Bouton rouge». Vous avez pu constater que les trois quarts de notre émission étaient consacrés à des chanteurs anglo-saxons. Pourquoi? Simplement parce qu'il ne s'est rien passé de très nouveau en France depuis plusieurs mois. Il y a cinq ans, par exemple, quand le rock a démarré ici, ça a vraiment bougé. Rappelez-vous, Vince Taylor... (Prise I)

Si on ne parle plus de Vince Taylor, c'est peut-être parce que les commerçants du disque veulent aller trop vite. Peut-être aussi parce que ça les gênerait réellement. Voilà comment aujourd'hui on fabrique un musicien. (Prise 2$)^{5}$

Sur ces quelques descriptifs et citations, on voit comment Garrel, représentant au $\mathrm{XX}^{\mathrm{e}}$ siècle de l'irréductible bohème contestataire emblématisée par Rimbaud et Verlaine, parvient à créer un espace de liberté dans un monde audiovisuel dirigé par l'État et par les puissances d'argent. On y retrouve ses motifs constants, la jeunesse, le couple, la drogue, le dandysme, la révolte, le rêve, l'anarchie. On y observe ses traits stylistiques majeurs, le portrait féminin, le minimalisme, la capacité de transformer tout protocole même aussi ingrat qu'un entretien sur l'argent en poème lyrique. On y constate un réflexe permanent de tout mettre en scène et en fiction, même les reportages. On y découvre une dimension peu présente dans ses films de cinéma, pleins d'esprit mais pas de rire: le burlesque (Ronnie et les mots). On y repère un dialogue constant avec les

5. En I96I, V. Taylor avait en France fait l'objet d'un lancement fulgurant par un entrepreneur de l'industrie musicale, et quatre ans plus tard s'était «suicidé» professionnellement en direct sur une scène parisienne, à un moment où il n'était pas encore à la mode de tout casser lors des concerts. 
images de Godard, pour des rectifications admiratives et iconoclastes sur la représentation de la jeunesse. On y assiste à la naissance d'un monde d'images gouverné par la musique pop. On y devine se dessiner, déjà, une place de souveraine pour Nico, qui vient d'être aimée et filmée par un autre pionnier de la contre-culture européenne, Peter Whitehead (clip I'm Not Sayin', 1965).

\section{LHeptatyque de Nico}

La rencontre avec Nico pendant le tournage du Lit de la Vierge va permettre à Garrel de concentrer les archétypes jusqu'alors explorés tour à tour de façon dispersée sur une seule figure. Poétesse, muse, archange, sphinx, poupée, prêtresse, déesse, Nico représente l'idole authentique et primitive, l'incarnation moderne des mystères de la création et de la mort, celle qui autorise les déclinaisons figuratives, l'Ur-Idol d'un culte païen exclusif mais panthéiste. Il n'est dès lors plus besoin de fable, le principe même d'un schème narratif s'avérerait trop limitatif. Comme s'y est employé Andy Warhol pour ses Superstars et modèles anonymes, il suffit de filmer Nico, mais chez Garrel, la contemplation du visage lisse et impénétrable de l'idole contient toutes les fables et libère beaucoup plus que tous les récits: l'univers infini de la poésie. L'Heptatyque de Nico (comme on dit le Polyptyque du Jugement dernier) fusionne spontanément le néoplatonisme, le romantisme et les exigences de liberté formelle propres aux années 1960 et 1970. La figure s'avère si puissante que les formes de son traitement peuvent, sans la léser, s'appliquer telles quelles à d'autres fétiches, telle Jean Seberg dans Les Hautes Solitudes (I974). Le seul film narratif de la fresque sera Voyage au pays des morts en 1976, fable et iconographie gothique exacerbées, comme si, au cours de l'exploration des formes contemplatives, il fallait qu'un panneau expérimente la force du récit, au même titre que La Cicatrice intérieure (197I) explore la force du plan d'ensemble, Un ange passe (1974) celle du montage parallèle, Le Berceau de cristal (1975) celle du gros plan, Athanor (1972) et Le Bleu des origines (1978) celle des images immobiles et muettes.

Même si une telle recherche relève de nombreuses sources cinématographiques, à commencer par la première période filmique de Warhol et les films allégoriques anarchistes de Lajournade, la principale inspiration formelle en reste bien sûr la musique de Nico elle-même. Ses albums produits par John Cale, The Marble Index (1969), Desertshore (1970) et The End (1974), transposent dans l'univers de la pop les propositions sérielles 
de Karlheinz Stockhausen. Présentes ou absentes, les nappes sonores de Nico imprègnent de leur rythme de mélopée les nappes optiques de Garrel et, à l'instar de l'œuvre de Stockhausen, les opus distincts de Nico et Garrel, films comme albums, revendiquent spontanément de ne former qu'un seul ensemble continu.

Prenons l'exemple de la structure d'Un ange passe, qui explore de façon troublante les puissances du montage parallèle. Deux types d'ensembles séquentiels se succèdent au long du film : d'une part, des portraits de Nico silencieuse; de l'autre, des séquences de dialogues entre couples, Laurent Terzieff et Maurice Garrel, Maurice Garrel et Bulle Ogier, Bulle Ogier et Jean-Pierre Kalfon. Ces deux versants du film ne se conjoignent jamais, leur rapport reste implicite, mystérieux, suspendu. Qu'est-ce qui passe, pourtant, de part et d'autre de la schize? Au moins cinq phénomènes. D'abord, la musique en off, les chants de Nico, qui parfois se prolongent jusque dans les séquences où elle ne se trouve pas; ensuite, son silence, si violent qu'il semble parfois effacer les paroles des interlocuteurs pourtant peu loquaces - mais chaque fois que leurs voix s'estompent, la mutité de Nico revient. Puis, une forme visuelle, le portrait, que Garrel en ces années I970 mène à son apogée d'intensité optique (durée des plans, fixité de la sidération, minimalisme, sérialisme), comme s'il prenait le relais des premiers films de Warhol, juste là où celui-ci avait abandonné son génie descriptif pur au profit des scénarios logorrhéiques de Ronald Tavel.

Entre les deux pans du film passe surtout une question séminale, celle de la création. Quel serait le rapport entre le visage de Nico silencieuse éperdument filmé et sa musique? Elle s'en souvient, elle la prépare, elle la médite, elle se laisse envahir, elle s'en repose? Réciproquement, la musique ne nous dicterait-elle pas de trouver ce visage immobile si intense? Or, les séquences de parole entre Maurice Garrel, Laurent Terzieff, Bulle Ogier et Jean-Pierre Kalfon représentent toutes les formes d'esquisse possibles, elles montrent des comédiens au travail, s'apprêtant à jouer, en train de jouer, improvisant ou en plein jeu. De sorte que le lien maintenu comme intemporel, indescriptible et énigmatique entre Nico et son chant, le lien de la création, se déploie sous ses formes claires, matérielles, concrètes, du côté des comédiens, qui se tiennent dans la temporalité perpétuellement inchoative d'une genèse. Mais retrouver ici le couple de L'Amour fou (Rivette, 1967), Ogier et Kalfon, montrer l'amitié de ces acteurs en grande intelligence de leur jeu, qui interprétèrent autrefois le rôle d'acteurs ravagés d'amour l'un pour l'autre, ne va pas sans conséquence. Leur coprésence spatiale en quelque sorte répare la schize et leur travail inté- 
gral - ils ont joué, ils vont jouer, ils jouent ensemble - suture la cicatrice générale du film. Un couple mythique coexiste, se reforme; quelque part hors champ quelqu'un leur demande sans cesse de se rapprocher l'un de l'autre; au fond de la séparation on a retrouvé l'éternité simple de l'amour.

Un ange passe assure une première fin de film classique, à la manière d'un conte de fées dont les fées seraient les formes modernes de la poésie. Nico joue, en concert, là-bas au loin sur la scène, prise dans un faisceau immense de lumière blanche, elle devient l'image syncrétique de la poésie: musicienne, poétesse, actrice et prêtresse. Quant à la fin seconde, celle qui concerne les comédiens, elle sublime l'inachevé sous forme de chute: plaqués devant une baie immense qui les dévore de sa lumière, Laurent Terzieff et Maurice Garrel se parlent en récitant qui du Rilke, qui le haïku le plus court du monde: «Un homme tombe. Bruit d'eau» (rappelons qu'un haiku comporte par définition trois vers ${ }^{6}$ ). Pour en arriver là, il fallait donc inventer une nouvelle structure, qui manifeste à la fois le silence profond présidant à la démiurgie et le souffle concret, parfois haletant, alimenté d'hésitations et d'angoisse, qui caractérise le travail. "Je ne sais si Gustave Moreau a senti combien cette belle conception du Poète-femme était capable de renouveler un jour l'économie de l'œuvre poétique ellemême. $\gg^{7}$ L'absence de lien assure le lien le plus fort; la juxtaposition tranchante des mondes, celui de la musicienne inspirée et celui des comédiens en recherche, organise une litote dictée par les exigences de l'admiration. Quel autre poète s'est livré à un éloge aussi subtil d'une artiste femme? Peutêtre Marcel Proust, pour la comtesse de Noailles - mais l'éloge était un peu mondain. Peut-être Sergueï Eisenstein, pour l'actrice Olga Kokhlova - mais c'était juste une analyse. Peut-être Louis-Ferdinand Céline, pour Arletty - mais c'était juste un scénario. Alors, personne: seul Garrel, pour Nico.

\section{Césure}

Without a guide, without a hand

Unwed virgins in the land

Unwed virgins in the land

Tied up on the sand

Nico, «Secret side», The End

6. L'un des plus célèbres poèmes de Matsuo Bashô dit: «Vieille mare / Une grenouille saute / Bruit de l'eau.»

7. M. Proust, «Les éblouissements» [1907], Chroniques, Paris, Gallimard, I949, p. I79. 


\section{Le cinéma des répliques}

Privé de la présence réelle de Nico, le cinéma de Garrel va passer d'un style allégorique cultuel à des formes narratives de fiction de type aristotélicien - dont il ne faut pas oublier qu'elles s'avèrent absolument neuves chez lui, qui n'y avait encore jamais recouru. Introduction du texte, du dialogue, du personnage, du devenir, des rapports entre cause et conséquence, invention d'un réalisme endeuillé... L'Enfant secret (1979), Liberté, la nuit (1983), Rue Fontaine (1984) apprivoisent la contemplation et transferrent ses pouvoirs de sidération dans la coupe, dans le raccord lui-même. ${ }^{8}$ Les fables désormais s'attachent à créer des échos narratifs, des ombres portées, des répliques. L'Enfant secret, Elle a passé tant d'heures sous les sunlights (1984), J'entends plus la guitare (1990) transposent en effet le mythe Nico sous des formes terrestres, hantées mais individuées. Garrel filme les servants après avoir filmé l'idole, raconte l'histoire après avoir vécu l'événement, analyse son rêve après avoir expérimenté le cauchemar. L'Heptatyque de Nico enregistrait le présent dans son étrangeté funèbre; le cinéma des répliques va reconstituer le passé comme mythe éternellement présent.

Retourner au monde: Garrel décrit ce tournant comme un désir de retrouver un public.

Comme les films meurent si personne ne les voit jamais, Langlois les passait quand je lui demandais et ça donnait des salles très pures, que j’avais dû désirer au fond, de sorte que la Cinémathèque était le seul endroit où ma paranoïa d'artiste s'éteignait. Il y avait aussi quelques projections dans d'autres salles mais comme ce type de cinéma ne déplace pas les foules, j’avais toujours à peine de quoi survivre, de sorte que la première chose que j'ai faite quand Nico et moi nous sommes séparés, c'est d'écrire une histoire. ${ }^{9}$

Dans le passage des allégories célestes aux fictions terrestres, on retrouve, mutatis mutandis, le même passage que dans l'interprétation platonicienne de l'image entre l'eidôlon archaïque et la théorie de la mimésis tel que le décrit Jean-Pierre Vernant. Sur le versant archaïque, l'eidôlon est défini par Platon comme «un second objet pareil» (Sophiste, 240 a-b); de ce point de vue, l'image relève de la catégorie du même. Mais dans l'eidôlon, la présence se manifeste en même temps comme une irrémédiable absence:

8. Voir, à propos de Liberté, la nuit, N. Brenez, «Le premier plan», De la figure en général et du corps en particulier. L'invention figurative au cinéma, Paris-Bruxelles, De Boeck, I998, p.36I-363.

9. P. Garrel, T. Lescure, Une caméra à la place du caur, ouvr. cité, p.67. 
C'est cette inclusion d'un «être ailleurs» au sein même de l'être-là qui constitue l'eidôlon archaïque moins comme une image au sens où nous l'entendons aujourd'hui que comme un double, qui en fait non une représentation dans le for intérieur du sujet, mais une apparition réelle insérant effectivement ici-bas un être qui sous la forme momentanée du même se révèle fondamentalement autre. Pour la pensée archaïque, la dialectique de la présence et de l'absence, du même et de l'autre, se joue dans la dimension de l'au-delà que comporte, en tant que double, l'eidôlon, dans ce prodige d'un invisible qui pour un instant se fait voir..$^{10}$

Les valeurs de présence, de prodige et d'éloignement sont celles-là mêmes qui s'attachent à la figuration de la Nico réelle, comme matrice de toutes les chimères.

Au contraire, dans la mimésis, l'image relève d'une autre catégorie que celle qui détermine le statut de l'eidôlon, manifestation de ce qui est; l'image prend un caractère fictif et illusoire, elle reproduit ce qui parait tel qu'il paraît:

C'est en opposant le paraître à l'être au lieu de les associer dans des équilibres divers comme on l'avait fait avant lui que Platon confêre à l'image sa forme d'existence propre; définie comme semblance, l'image possède un caractère distinctif d'autant plus marqué que l'apparence n'est plus considérée désormais comme un aspect de la réalité mais comme une dimension spécifique posée en face de l'être dans un rapport ambigu de fauxsemblant. Cette spécificité implique en contrepartie l'expulsion de l'image hors de l'authenticité, sa relégation dans le champ du fictif, sa disqualification du point de vue de la connaissance. (ibid., p. I3I)

Plus profondément qu'un réinvestissement des formes narratives classiques, le passage de l'Heptatyque de Nico au cinéma des répliques marque donc une saute qualitative quant au statut de l'image, d'ordre quasi anthropologique: on est passé de l'eidôlon au simulacre, du totem aux jeux de la semblance. Une telle saute va autoriser l'ensemble des dédoublements qui structure Elle a passé tant d'heure sous les sunlights et se propage continûment de film en film. Sur un mode négatif, en témoigne avec force l'absence de toute image d'archives de Nico dans les films terrestres. Depuis L'Enfant secret, pas une photographie, pas une pochette de disque, pas un rush, pas une empreinte analogique de Nico n'est entrée dans ces films que pourtant elle informe et hante. Il s'agit bien de deux mondes d'images incompatibles, appartenant au même univers mental mais séparés par une frontière symbolique jusqu'à présent infranchissable.

IO. J.-P. Vernant, «Naissances d'images», Religions, histoires, raisons, Paris, Maspero, 1979, p. III. 
Seule la musique a pu se frayer un chemin dans Elle a passé tant d'heures sous les sunlights et J'entends plus la guitare, comme si elle appartenait depuis toujours à un au-delà, qu'elle était destinée à infiltrer les vivants de ses puissances invocatoires.

\section{Les travailleurs de l'image. Danièle Huillet et Jean-Marie Straub par Pedro Costa}

Tout art nouveau pourrait être qualifié de montage Theodor Adorno, Théorie esthétique.

Au détour d'une séquence du Bellissima de Luchino Visconti (I95I), au fin fond de son calvaire dans Cinecittà, Maddelena (Anna Magnani) traverse une salle de montage, traitée en soute obscure où travaillent de pauvres créatures féminines exploitées. Contre cette vision industrielle du montage comme tâche subalterne effectuée par de petites mains, s'arcboute la lignée des films qui décrivent la salle de montage en ombilic de la création filmique: L'Homme à la caméra de Dziga Vertov (1929), le diptyque The Fall (1969) et Fire in the Water (1977) de Peter Whitehead, L'Automne de Marcel Hanoun (197I), Blow Out de Brian De Palma (198I), Scénario du film Passion (198I) et JLG/JLG-autoportrait de décembre (1995) de Jean-Luc Godard... Admirable essai sur la création, Oì git votre sourive enfoui? de Pedro Costa (200I) se trouve à la croisée de trois traditions : celle des films qui scénographient voire héroïsent la salle de montage transformée en objectivation d'un espace psychique (L'Automne de Marcel Hanoun par exemple s'y déroule entièrement); les films qui analysent images, motifs, raccords, photogrammes, intervalles à même la pellicule, tel le magistral Tom Tom the Piper's Son de Ken Jacobs en 1969; les films, encore peu nombreux, qui réfléchissent l'histoire des styles de montage, tel le très pédagogique Film sur le montage de Jean Mitry (1964), récemment remis en circulation par la Cinémathèque de Belgrade, ou la série télévisée La Lucarne du siècle réalisée par Noël Burch (1985).

Oì git votre sourire enfoui? associe plusieurs formes d'investigation sur le raccord: formes des enchaînements filmiques (étude sur les raccords des Straub); modes de description des passages entre plans (exposition des images par Costa); transferts entre image et idée (l'impératif de sens); voies du commerce humain, circulations tout à la fois objec- 
tives, affectives et souterraines entre les êtres. Un lien unit l'ensemble de ces chantiers aux résonances infinies: la précision, une précision dont il faut ressaisir les origines et les perspectives.

Les séquences s'enroulent chacune à leur tour autour d'un raccord, dont il s'agit d'observer, non pas l'émergence puisqu'il est déjà là - Straub et Huillet montent une troisième version de Sicilia!-, mais le polissage, le sens et les effets. Tour à tour, les figures d'un passage de séquence à l'autre, d'un champ-contrechamp (dialogue dans le train), d'un raccord dans l'axe (dialogue sur le parvis)... se déploient. Chaque fois, un paramètre différent vient habiter la décision de mettre fin à un plan ou un son et de commencer le suivant: la lumière, un sourire dans les yeux d'un acteur, un bruit de portière intempestif... Chaque fois, la coupe et l'enchaînement se déterminent au photogramme près: c'est donc à la naissance de la nécessité que nous assistons. Or, cette nécessité ne se dégage pas seulement du matériau lui-même: elle se fonde sur un ensemble de déterminations, de sources, d'exigences, qui situent chaque raccord straubien en pointe avancée de l'héritage de la pensée critique, des Lumières à Franco Fortini.

Où gît votre sourire enfoui? offre un essai documentaire sur la pensée critique telle qu'elle est incarnée et vécue par deux travailleurs de l'image, Straub et Huillet. Dans le corps à corps avec la pellicule, leurs décisions proviennent en effet aussi d'une passion cinéphilique, musicale, philosophique et artistique qui fournit modèles, points d'appui, références secourables - Les Contes de la lune vague hantent les paysages siciliens. Elles proviennent encore d'une exigence dialectique, exposée de façon limpide et concrète à propos du champ-contrechamp dans le train : à quelques photogrammes près, soit c'est le personnage, soit c'est le spectateur qui décèlera un mensonge; bien sûr la seconde option s'impose, Brecht gît enfoui dans le photogramme. Au-delà, les décisions créatives proviennent des idéaux et exigences matérialistes, c'est-à-dire d'une théorie de combat: guerre sans fin contre le monde qui a institué l'injustice comme état de nature, et lutte sans merci contre toutes les représentations qui s'évertuent à rendre cet état supportable voire désirable. Pour un raccord, pour un ensemble d'images, pour un film, faire sens suppose en chaque instance d'exécuter un geste critique contre la domination. Toute œuvre d'art constitue un laboratoire du sens, permet d'observer le fonctionnement des règles, du hasard, de la nécessité, de la décision, de l'organisation, d'une économie de la signification et de l'insignifiant, de la prise en charge ou en compte d'une extériorité... Pour une œuvre critique, il 
s'agit de contester les protocoles symboliques qui permettent au monde injuste de se perpétuer, à commencer par le statut secondaire et annexe de l'art comme d'un reflet. Où gît votre sourire enfoui? va donc centrer son effort sur la création comme intervention, comme acte, comme travail, comme praxis, à l'instar des Straub pour qui le cinéma et l'art - à la suite de Cesare Pavese, Elio Vittorini et même Charles Péguy que JeanMarie Straub cite ici - doivent participer à l'émancipation, donc notamment élaborer l'histoire de ceux qui n'ont pas d'histoire, les ouvriers, les paysans, les combattants.

Ainsi préside au choix précis de la coupe un ensemble de positions et de propositions non négociables, d'ordre polémique et pratique, qui fait de chaque geste artistique un acte de rupture contre la domination, et dont les Straub ont trouvé des répondants esthétiques par exemple dans la conception de la césure que Friedrich Hölderlin a exposée à propos des traductions de Sophocle ou dans les théories d'Arnold Schoenberg sur la composition musicale. ${ }^{\text {II }}$ Il se trouve que Theodor Adorno avait nommé «montage» l'esthétique pour laquelle faire œuvre signifiait détruire les fondements du monde dans laquelle volens nolens elle s'inscrit:

C'est pourquoi le principe de montage - avec une conséquence dont les degrés devraient être décrits par cette histoire de l'esthétique qui n'existe pas encore $-s^{\prime}$ est converti en principe de construction. ${ }^{12}$

Même si chez Adorno la notion de montage n'équivaut pas au montage de cinéma -elle s'apparente plus au collage, en ce sens qu'elle privilégie l'hétérogène -, le montage adornien, qui cultive dissonances et blessures, équivaut bien aux choix des Straub et de Costa.

Le cinéaste est donc traité ici en artisan, dépositaire d'un savoir-faire qui, justement parce qu'il relève d'une longue tradition philosophique et politique concernant la praxis, requiert de celui qui a le bonheur de l'observer la même intensité et le même sérieux pour en transmettre quelque chose à son tour. Aux raccords impératifs des Straub répond la façon dont Costa organise l'exposition de leurs plans. Une syntaxe plastique à la diversité didactique, élégante et rigoureuse s'organise : image plein cadre, filmée comme plan, filmée comme motif, filmée comme lueur de loin sur l'écran

I I. F. Hölderlin, «Remarques sur les traductions de Sophocle» [I804], Euvres complètes, traduction P. Jacottet, Paris, Gallimard, 1967; A. Schoenberg, Fondements de la composition musicale [1967], traduction D. Collins, Paris, Lattès, I987.

I2. T. W. Adorno, Théorie esthétique [1970], traduction M. Jimenez et É. Kaufholz, Paris, Klincksieck, I989, p.83. 
de la table de montage; grappe de photogrammes vus comme séquence, comme série d'intervalles, comme possibilité d'arrêts, de retours et de prélèvements; entrée et sortie des plans de Sicilia! dans le film de Costa, à la manière dont Robert Mitchum entre et sort dans les espaces de La Nuit $d u$ chasseur (Charles Laughton, I955) - un événement, une épiphanie noire. La syntaxe d'exposition des plans s'enrichit encore des modes de refilmage sur écran de La Chronique d'Anna Magdalena Bach (1968) projeté dans l'amphithéâtre du Fresnoy: l'image devient une présence lointaine qui a toujours été là, qui sera toujours là après nous, qui déborde l'existence actuelle de l'ensemble de ses virtualités symboliques, elles-mêmes issues de l'invention d'une nécessité esthétique profondément réfléchie. L'anecdote célèbre que Straub raconte à propos du choix de Gustav Leonhardt - contre le producteur voulant spéculer sur la gloire d'un chef d'orchestre célèbre-, on le comprend désormais, peut s'appliquer au choix de chaque raccord et de chaque photogramme dans chaque film.

Pour étayer leur œuvre en nécessité, certains artistes s'appuient sur des modèles mathématiques, géométriques, organiques ou discursifs, qui leur fournissent des schèmes architectoniques et des repères de légitimation. Pour les Straub, le modèle structurant reste devant nous : c'est celui du communisme utopique, "celui qui pourrait sauver la terre», comme Jean-Marie Straub l'explique ici à propos de La Mort d'Empédocle (1987). L'histoire n'a pas été racontée, le socialisme n'a pas encore existé, la terreur capitaliste règne, les impératifs pressent. À l'ensemble des déterminations politiques prospectives qui préside aux gestes artistiques des Straub, répond le traitement du temps, de l'espace et des corps chez Costa. En dépit de son étroitesse, l'espace de la salle de montage n'est pas plus totalisé que celui de la cellule du Condamné à mort de Robert Bresson (1956); pour être circonscrit à certains raccords, le temps de la création ne s'ordonne pas à la fabrication d'un film, dont ni le début ni la fin ne nous sont livrés. Plus ils sont centrés, approfondis et minimaux, plus les éléments deviennent ouverts, sans unité de temps ni d'espace ni d'action. Les principes compositionnels s'avèrent strictement endogènes à l'essai : seule la question du raccord fait noyau, seule la cartographie de la représentation des images fait unité.

De la même façon restent profondément ouverts les corps, les êtres, le couple. Sur les figures se déploie un travail systématique de l'intervalle. Au moyen de plans fixes - profils de camée, dos majestueux, mains monumentales -, Costa crée des effets de portraits immobiles, autonomes, contemplatifs (jusqu'à la mélancolie d'un Rembrandt tardif dans le plan 
final de Straub assis sur les marches); mais simultanément, par répétition (en particulier du profil noir sur noir de Straub), décalque, variations sur motif, effet de manque (il faudra attendre les 6 Bagatelles qui viendront en annexe de Oì gît votre sourive enfoui? pour découvrir Straub et Huillet assis côte à côte face à la table de montage), il affirme la matérialité sérielle du document visuel: le battement du noir et de la lumière affecte les corps autant que les photogrammes. Straub et Huillet sont en flicker, avec eux-mêmes, entre eux, pas des psychologies mais des organismes cinématographiques, qui le savent et s'en réjouissent, tel Straub entrant et sortant sans cesse de la pièce pour incruster dans le documentaire de Costa un petit moment de Lubitsch. À la manière d'un sourire, Costa lui répond formellement en montant sur un plan fixe de son profil le rugissement léonin de la pellicule que l'on rembobine sur la table de montage. Autrement dit, la série illimitée des portraits autonomes ne se referme pas sur une identification individuelle, il ne s'agit aucunement de portraiturer des personnalités, même si on ne les a jamais aussi bien vues; il s'agit de manifester une nécessité à l'œuvre. La possibilité de telles ouvertures ontologiques naît bien sûr du noir, dont la masse plastique règne sur Où gît votre sourire enfoui ? Un noir non pas de l'énigme, du secret insondable de la démiurgie - tout au contraire, un noir matérialiste qui s'ancre concrètement dans l'intervalle photogrammatique; se prolonge dans l'obscurité matérielle indispensable au visionnement des plans; correspond au silence de la réflexion (colère de Huillet, quand le bruit l'empêche de se concentrer); et laisse sa part visible à la négativité historique et esthétique dont l'œuvre des Straub a déduit sa dynamique.

La seule trace de composition classique qui parcourt Oì gît votre sourive enfoui? concerne l'amour. Une évolution affective souterraine mais linéaire nous mène des violents reproches adressés par Huillet à Straub, à une série de compromis pratiques passés au bénéfice de l'ouvrage, à un réseau de signes empathiques souvent accrochés à des jeux de citations cinéphiliques, et se conclut par une déclaration d'amour fou de Straub pour Huillet. Soudain, une fin de film explosant d'amour, qui renvoie à leur caractère inane et aliénant tous les happy ends frelatés du cinéma de la domination. Où gît votre sourire enfoui ? pulvérise de sa beauté les falsifications sentimentales dont nous écœure le cinéma industriel.

La même exigence matérialiste traverse Adorno, les Straub et Costa: une passion pour le travail émancipateur, dont on trouverait certaines origines historiques dans les textes de Karl Marx et de Friedrich Engels sur la pensée des ouvriers, qui donneront naissance au principe d'«éthique 
prolétarienne». Or, contrairement aux interprétations réductrices, chez Marx, l'éthique prolétarienne n'attribue nullement l'exclusivité de la pensée critique à une classe sociale, elle noue une solidarité entre l'injustice, la souffrance et la pensée. En mai I843, Marx écrit dans une lettre adressée à Arnold Ruge :

L'existence de l'humanité souffrante qui pense, et de l'humanité pensante, qui est opprimée, deviendra nécessairement immangeable et indigeste pour le monde animal des philistins, monde passif et qui jouit sans penser à rien. C'est à nous d'amener complètement au grand jour l'ancien monde et de former positivement le monde nouveau. Plus les événements laisseront de temps à l'humanité pensante pour se ressaisir et à l'humanité souffrante pour s'associer, et plus achevé viendra au monde le produit que le présent abrite dans son sein. ${ }^{13}$

Straub, qui tournait en I6 millimètres pour pouvoir montrer ses films dans les usines, a parfois tenu des propos radicaux contre "l'humanité pensante» réduite à la corporation des intellectuels :

Je maintiens, comme Glauber Rocha, que le cinéma précisément est fait pour les ouvriers et les paysans, qu'il répond à leur nécessité vitale. Le cinéma tient sa force du vécu quotidien des paysans et des ouvriers, alors que les intellectuels n'ont aucune expérience, il faut savoir qu'ils ne vivent même pas. C'est pourquoi les films ne signifient rien pour eux, quand d'autres y trouvent ce qui les préoccupe et ce qu'ils doivent surmonter, au jour le jour. ${ }^{I 4}$

Costa, plus fidèle en cela à Marx que les Straub, et qui dans Tarrafal (2007), son film le plus straubien à ce jour, traitera les immigrés clandestins en dialecticiens méditatifs, récuse la division non pas, banalement, entre ouvrier et intellectuel, mais entre pensée manuelle et pensée conceptuelle: la table de montage est le site tranquille de cette évidence qui exige pourtant reconquête. Où gît votre sourire enfoni ? atteste, documente et réfléchit la figure du cinéaste en artisan communiste, et de ce fait accomplit la fusion concrète que visait sur un plan théorique le concept de praxis. S'il s'agit de donner une histoire au travail, alors l'essai de Costa en constitue une pierre blanche: Straub et Huillet apparaissent ici sans la naïveté mais avec la même simplicité que le cinéaste amateur prénommé Marcel dont Godard comparait le travail minutieux et dévoué avec celui

I3. K. Marx, Euvres, t.III: Philosophie, traduction et édition M. Rubel, Gallimard (la Pléiade), Paris, 1982, p.342. Nous soulignons.

I4. Table ronde avec G. Rocha, P. Clémenti, M. Jancso (1970), dans Cinéma/Politique. Trois tables rondes, Liège, Labor, 2005 , p. 44. 
des ouvriers horlogers dans l'épisode « $3 \mathrm{~b}$ » de Six fois deux (Sur et sous la communication) (1976). Dans la petite salle de montage, autour de l'observation de quelques figures de raccords, s'est élaborée une zone temporaire d'amour révolutionnaire. "L'art maintient cette utopie au sein de l'irréconcilié. $\gg^{\mathrm{IS}}$

\section{Détournement de l'idôlatrie: Chic Point de Sharif Waked}

Chic Point. Fashion Show For Israeli Checkpoints, du cinéaste arabe israélien Sharif Waked, est un bref pamphlet visuel de 7 minutes tourné à Tel-Aviv en 2003, qui réinvestit entièrement l'iconographie et le dispositif de l'un des rituels d'adoration commerciaux les plus emblématiques de la société du spectacle: le défilé de mode. Au son d'un morceau du musicien activiste Leftfield ("Song of life», sur l'album Leftism, I995), des mannequins masculins défilent dans un espace abstrait, sur fond noir, avançant sur un podium imaginaire dont nous serions les spectateurs. Ils présentent d'étranges vêtements, découpés de trous, de Zip, de filets, de résilles à la place du torse. Des vêtements que l'on peut ouvrir facilement comme si le corps était une valise - l'un d'entre eux possède une ouverture en forme de cintre - ou qui sont déjà ouverts, laissant visible la peau. À cette partie moderne, mobile et apparemment frivole, en couleurs, vidéo et musique, succède une série de photographies en noir et blanc, dans un épais silence: aux checkpoints de Gaza, Naplouse, Ramallah ou Jénine, des habitants palestiniens, seuls ou en groupes, soulèvent leurs chemises, leurs polos, leurs vestes, pour que des soldats israéliens puissent vérifier qu'ils ne portent pas d'armes ou de ceintures d'explosifs. Dans les formes d'écartement des tissus, on reconnaît alors le patron des modèles burlesques inventés par Waked pour son défilé. La transposition ironique a d'abord été donnée, par adoption moqueuse de l'humiliation quotidienne; puis le référent concret: terreur militaire et oppression politique.

Le checkpoint, en Israël, possède au moins une double fonction: à court terme, se protéger contre d'éventuels attentats kamikazes; à long terme, déchirer et diviser tellement le territoire que l'existence d'un État palestinien, si un jour elle possède une effectivité juridique, puisse difficilement recouvrir une réalité physique et géographique. Les trous et les larges mailles des modèles inventés par Waked reproduisent aussi les déchirures

I 5. T. W. Adorno, Théorie esthétique, ouvr. cité, p. 54. 
du territoire palestinien, lacéré en bandes, villages, morceaux et bouts qui chaque jour raccordent plus mal les uns avec les autres. La Palestine devient ce pays où tout n'est plus que frontière avec elle-même; à l'exil extérieur se superpose une quotidienne et paradoxale expulsion intérieure.

Chic Point élabore en images un petit traité de la déchirure. Le film s'édifie sur des oppositions très franches et qui s'accentuent mutuellement, parmi lesquelles: le mobile de la vidéo contre l'immobile de la photographie; la couleur vidéographique contre le noir et blanc des images fixes; la musique ambiant du monde de la mode contre le silence total du monde politique; la frivolité du fashion show contre le caractère tragique de la répression militaire; le contrat d'attirance et d'attraction qui s'attache à un défilé de mode, contre l'aversion et l'hostilité qui se manifestent aux checkpoints; le caractère abstrait de l'espace vidéographique (juste du noir, on ne voit ni podium ni aucun décor) contre la précision spatiale et temporelle des incidents et arrestations aux checkpoints; la continuité fluide des corps apparaissant pour se présenter au visible contre la discontinuité sèche des photographies qui se succèdent; l'étrangeté onirique des costumes contre la quotidienneté concrète et aride des situations répressives; le regard absenté professionnel des mannequins (éviter qu'il y ait trop d'échange, on se trouve dans un au-delà du regard) contre les yeux bandés des Palestiniens en état d'arrestation (plus de vision possible, on se trouve en deçà du regard).

Sollicité par la violence sensible du contraste entre les deux parties, l'esprit du spectateur s'active simultanément à propos de la nature même d'une telle fracture. Plastiquement, la fracture entre les deux versants du film est une frontière, elle s'impose au titre d'une démarcation entre deux régimes d'images (vidéo et photographie). Formellement, elle est une césure, elle reproduit au sein même de la syntaxe du film les motifs d'entailles, de fentes, de blessures, de cicatrices, qui s'attaquent aux vêtements et, de là, aux corps et au territoire. Émotionnellement, elle est une rupture, elle travaille sur la violence spéculative qu'il faut infliger à l'esprit pour surmonter une contradiction. Dialectiquement, elle suggère un saut qualitatif, elle indique une sortie possible hors de l'histoire (penser un ailleurs et un autrement vis-à-vis d'une guerre sans fin).

On peut noter encore que certains éléments traversent discrètement les deux versants du film. Les corps et postures athlétiques de deux Palestiniens arrêtés, yeux bandés, mains ligotées derrière eux, renvoient spontanément à des poses attiques qui répondent aux mêmes modèles de beauté classique que celles des mannequins. De part et d'autre de la 
césure, toutes les présences sont masculines; et enfin, la parole, le discours sont mis hors champ dans les deux contextes. Le principe d'étanchéité entre deux univers, quel qu'en soit l'antagonisme (espace, temps, système symbolique), s'avère donc esthétiquement intenable et impossible.

Dans le contexte d'une guérilla permanente et multiforme qui dure depuis soixante ans entre Israéliens et Palestiniens, au lieu de travailler sur le mode mécanique de l'attaque et de la contre-attaque, Chic Point transforme la réplique en riposte caustique. Son ironie brillante rappelle les trouvailles ingénieuses des cyniques grecs, ces philosophes athées, pauvres et insolents qui pensaient autant avec leurs corps qu'avec leurs mots. Par exemple, Grégoire de Naziance rapporte ce trait d'esprit inventé par Antisthène :

Quel grand homme que cet Antisthène! Frappé en pleine figure par un de ces voyous impudents, il se contente en retour de tracer sur son front le nom de son agresseur comme sur une statue le nom de l'artiste - de façon probablement à accuser l'autre de manière plus cuisante. ${ }^{16}$

Voilà, selon Chic Point, le principe de toute victoire réelle, quel que soit l'état d'oppression dans lequel se trouve encore la victime: la virtuosité de son ironie prouve la supériorité morale qui par avance vainc un ennemi déjà considéré comme un interlocuteur et non pas comme une cible. Quoi que l'histoire collective lui réserve, Chic Point a vaincu, non pas un ennemi singulier, mais la guerre elle-même. Waked habite un pays sans frontières, un pays sans aucune limite qu'il aura créé à force d'esprit:

Ma patrie n'est pas faite d'une muraille ni d'un toit, Mais la terre entière est la cité et la maison Mise à notre portée pour y habiter à demeure..$^{17}$

Les propositions esthétiques de Philippe Garrel, de Pedro Costa et de Sharif Waked, chacune à leur manière, réactualisent les sources romantiques de l'humanisme révolutionnaire. Leurs œuvres respectives renvoient à Friedrich Schiller, à Friedrich Schlegel, aux poèmes du jeune Karl Marx, aux réflexions de Walter Benjamin sur la violence historique, à tous ceux qui refusèrent de dissocier les luttes réelles et le travail de la pensée. Un «ancêtre secret» aujourd'hui minoré pourrait en être Ludwig Feuerbach, le premier philosophe allemand à avoir rejeté la tradition

I6. Les Cyniques grecs. Fragments et témoignages, Ottawa, Éditions de l'Université d'Ottawa, 1975, p. 55 .

I7. Ces vers sont tirés d'une tragédie perdue de Cratès, autre philosophe cynique: ibid., p. I2O. 
idéaliste et prôné un nouveau commencement de la philosophie à partir du réel. Rappelons sa Thèse provisoire $n^{\circ} 28$ :

Le commencement de la philosophie n'est pas Dieu ni l'absolu ni l'être comme prédicat de l'absolu ou de l'idée - le commencement de la philosophie est le fini, le déterminé, le réel. ${ }^{18}$

Mais qu'est-ce que le réel? La souffrance, «la misère humaine ».9 Quel est alors le rôle de l'art? L'apprentissage du sensible. Thèse provisoire $n^{\circ} 39$ :

Si on l'exprime correctement, la proposition: l'art «représente la vérité sous forme sensible» [proposition de Hegel] veut dire : l'art représente la vérité du sensible. (p.183)

Or, le sensible n'équivaut aucunement à ce qui serait donné, immédiat, et devrait être dépassé; mais à ce qui doit faire l'objet d'un long apprentissage pour être saisi, compris, purifié aussi des projections que le sujet lui impose. L'art est ce long accès à la complexité et à la beauté du réel. Feuerbach ne développe pas une conception de l'art béate et euphorique (les choses seraient là, il n'y aurait qu'à les contempler), mais une conception hautement mélancolique qui, grâce aux textes de Benjamin, nous est depuis devenue familière. Thèse provisoire $\mathrm{n}^{\circ} 22$ :

L'art procède du sentiment que la vie d'ici-bas est la vraie vie, que le fini est l'infini. (p. 109)

Thèse provisoire $\mathrm{n}^{\circ} \mathbf{2 3}$ :

La conscience de la divinité de l'humain, de l'infinité du fini, cette conscience résolue, devenue chair et sang, est la source d'une poésie et d'un art nouveau, qui surpasseront tous les arts précédents en énergie, profondeur et flamme. La foi dans l'au-delà est une foi absolument contraire à la poésie. C'est la souffrance qui est la source de la poésie. Celui-là seul qui éprouve une perte infinie dans la perte d'un être fini, peut concevoir les feux du lyrisme. Seul le charme douloureux du souvenir, de ce qui n'est plus, est le premier artiste, le premier idéaliste dans l'homme. (p. IIO)

On sait comment Marx reprendra de Feuerbach et contre lui (dans le fameux $\mathrm{Ad} \mathrm{Fenerbach}{ }^{\circ}$ ) le même mouvement d'arrachement et de renversement par lequel la philosophie devra sortir d'elle-même pour devenir praxis. Les images de Garrel, Costa, Waked nous semblent procéder

I8. L. Feuerbach, Thèses provisoires pour la réforme de la philosophie [1842], dans Manifestes philosophiques, textes choisis et traduits par L. Althusser, Paris, PUF, I973, p. III.

19. L. Feuerbach, Principes de la philosophie de l'avenir [1843], ibid., p. I27.

20. K. Marx, Ad Fenerbach. Thèses sur Feuerbach [1845], dans Euvres, t. III, ouvr. cité, p. IO29 et suiv. 
de ce mouvement d'arrachement et de recommencement de l'art à partir d'un protocole critique, ce geste de décollement qui arrache la représentation à elle-même pour que, d'enregistrement d'une trace de corps, elle devienne intervention spéculative sur la présence. Comme le formule superbement Garrel: "Il y a une solidarité des artistes véritables et des révolutionnaires, parce qu'ils refusent les identifications ordinaires. ${ }^{2 \mathrm{I}}$

2 I. P. Garrel, T. Lescure, Une caméra à la place du cour, ouvr. cité, p. I44. 


\section{::: \\ Des corps sans visages: ce que le cinéma fait avec les foules}

L'écran cinématographique est anthropomorphe, saturé de vie humaine. Lorsque la caméra, tel un œil inquisiteur et silencieux, révèle un paysage, suit un animal, observe une machine, décrit une installation, des commentaires semblent inutiles. Il est pourtant rare que n'intervienne pas une voix, celle d'un faux naif questionneur, d'un expert, d'un usager qui détaille à notre intention ce que nous sommes justement en train de voir. Ces bavards ne nous renseignent pas, ou très peu; leurs explications, souvent redondantes, réinstallent, aux côtés des choses, la nécessaire présence des corps.

Le cinéma ne fait pas que raconter des histoires, mais il montre toujours des personnes, ou plutôt des jeux entre personnes. Deux, trois individus occupent un cadre que leur proximité rend étroit. Rien n'interdirait de miser sur l'espace, de laisser une centaine de mètres entre les personnages, mais - on s'en rend compte dans les scènes de duel au pistolet, prises en enfilade, jamais perpendiculairement - le vide central accaparerait les regards. Dans le champ, les acteurs doivent être au contact les uns des autres, parce qu'il leur faut travailler ensemble. L'art d'un comédien consiste à donner physiquement la réplique aux corps qui se trouvent en face de lui, à s'adapter, sans y réfléchir, à leurs gestes, et à les guider tout en les suivant. On nomme «jeu naturel» la mise en œuvre d'une convention grâce à laquelle se trouvent éliminées les réactions spontanées, le recul, l'élan, l'écart, tous ces réflexes au moyen desquels un individu manifeste, d'ordinaire, le léger flottement que provoque chaque nouvelle rencontre. Réfléchissant au burlesque, Jean-Louis Schefer avance une très heureuse remarque: le comédien, écrit-il, «doit gérer l'espèce de reflux 
des inventions du scénario sur sa pratique gestuelle $»^{\mathrm{I}}$; au lieu d'être luimême, il lui faut agir avec et contre le personnage qu'on lui impose.

Ce que nous tenons pour naturel est une correspondance entre des appels et des réponses, fruit d'un apprentissage. Une performance approche d'autant mieux la perfection que les traits de l'acteur, ses gestes, son attitude réussissent, sans recours au langage, à poser un événement. Pensons à une scène classique, cent fois reprise: deux personnages s'entretiennent et la figure de l'un d'eux, passant du sourire à la crispation puis à la détresse, met en scène toutes les étapes d'une défaite. Béla Balász avait senti ce privilège du visage où, pour lui, se concentrait la force expressive d'un acteur:

La mimique manifeste des sensations et par là elle est lyrique. D'un lyrisme dont l'éloquence est incomparablement plus riche, plus variée que celle de n'importe quelle littérature [...]. Sur un visage peuvent apparaitre ensemble les choses les plus diverses et, comme dans un accord musical, le rapport entre ces traits fait naitre les plus riches modulations [...]. Le premier plan d'un visage doit être une synthèse lyrique du drame tout entier [...]. Nous devons pouvoir nous adonner longuement à sa contemplation pour arriver à lire en lui. ${ }^{2}$

Le visage-énoncé représente, il traduit une évolution davantage qu'il n'extériorise de purs affects. À la différence d'un visage ordinaire, il est une construction artistique dont on mesure la vigueur et le caractère artificiel quand on le confronte à celui qu'exhibent des amateurs s'ils se trouvent face à une caméra. Menschen am Sontag, réalisé en 1929 par Robert Siodmak et Edgar Ulmer, évoque un dimanche berlinois, calme et ensoleillé, en mariant des scènes de rue à des séquences où quatre inconnus jouent leur propre rôle au cours d'une excursion à la campagne. Impressions et émotions se manifestent directement sur leurs visages, en une fois, sans nuance ni transformation; leurs gestes se limitent à poser des actes; ils sont trop frustes, trop immédiats pour que le public songe à lire sur leurs traits les moments d'une histoire.

Le corps cinématographique est le lieu où trouvent forme des destins individuels, il condense des vies artificielles, mises en scène dans le cercle étroit que construit la fiction, et, grâce au travail du visage, les agrémente d'une forte charge psychologique. Privilégiant les jeux de physionomie, le cinéma n'a aucun besoin de corps quelconques, spontanés et naïfs. Les

I. J.-L. Schefer, Images mobiles, Paris, POL, 1999, p. I49.

2. B. Balász, Der sichtbare Mensch oder die Kultur des Films, Vienne-Leipzig, DeutschÖsterreichisches Verlag, 1924, p. 65, 67 et 7I (nous traduisons). 
comparses qui, très souvent, meublent l'arrière-fond demeurent à peu près invisibles, et n'ont, en tout cas, qu'une très faible importance narrative. C'est pourtant à de telles silhouettes, sans noms et sans histoire, que je voudrais ici m'intéresser: aux anonymes, aux foules denses, peu différenciées, fortement présentes, que la caméra saisit quand elle cherche à capter le monde dans lequel nous vivons, qui nous entoure et où elle se déplace, aux masses compactes et mouvantes qu'on fait évoluer au loin, derrière les acteurs, tous individus qui ne sont rien et doivent néanmoins servir à quelque chose puisqu'on rétribue leurs services.

\section{Corps baladeurs}

Parler $d u$ cinéma signifie, sauf indication contraire, s'exprimer au sujet de la fiction; les films que, pour aller vite, j'appellerai documentaires sont rarement pris en compte, à moins que leur réalisateur ne porte un nom illustre, Flaherty, Ivens, Antonioni, Buñuel. Ils ont pourtant tenu un rôle important dans l'évolution du cinéma, ils ont été l'école à laquelle se sont formés la plupart des «grands» cinéastes, ils ont permis aux studios de tourner, aux techniciens de travailler sans interruptions et, parce qu'ils ne coûtaient pas cher, ils ont trouvé une place dans les programmes. Le documentaire ne se définit pas à travers sa fidélité au réel; nous avons noté que plusieurs séquences de Menschen am Sontag ont été jouées: le fait n'a rien d'exceptionnel, chacun de nous, placé face à une caméra, tend à mimer ce qu'il croit être sa propre image. Ce qui distingue le documentaire de la fiction est l'absence de figures connues, la lâcheté de la structure narrative et surtout la présence de groupes anonymes. J'ai insisté sur les conventions grâce auxquelles, dans le cinéma narratif, corps et visages sont à la fois des destinées et des histoires. Le documentaire ne se prétend pas plus authentique que la fiction et, quand il montre des personnes, il se plie également à des normes, il se sert des corps davantage qu'il ne les sert, mais ses formules relèvent d'une autre logique, et c'est cela que je voudrais mettre en évidence.

Le cinéma, forme de spectacle destiné à un public nombreux, constamment renouvelé, débuta en montrant aux spectateurs ce qu'ils étaient eux-mêmes, des points, des formes au milieu de la foule urbaine. Ce fut, note Schefer, "avec l'invention, l'évolution ou parfois la perversion de la découverte du spectateur qu'il commença son exploitation ».3 À la même

3. J.-L. Schefer, Du monde et du mouvement des images, Paris, Cahiers du cinéma, I997, p.39. 
époque, à travers l'Europe, un ensemble d'essais parfois sérieux, parfois simplement polémiques, faisaient naître l'idée que le rassemblement d'une grande quantité de personnes constituait une entité originale, irréductible aux individus qui la composaient, ayant ses règles propres de fonctionnement. Il n'y a pas là une simple coïncidence : le cinéma, divertissement proposé à la foule, contribua à fixer une notion encore imprécise. Une caricature publiée dans un magazine de cinéma viennois montre, à gauche, une masse de curieux agglomérés autour de quelque chose qui nous échappe et, à droite, deux hommes isolés; l'un d'eux suggère: «Allons nous informer sur ce qui se passe », l'autre répond: «Je le verrai demain au cinéma. ${ }^{4}$ Le dessin traduit parfaitement le rapport entre la vie des rues, sa transposition à l'écran et la mise en évidence du phénomène grégaire à travers le film. En conduisant des masses de badauds vers un lieu où ils allaient, ensemble, assister à une projection, et en représentant ces mêmes badauds, le cinéma conféra au mot foule l'épaisseur d'un cliché.

Je vais tenter d'éclairer la mise en place de cette image, mais, auparavant, je voudrais m'arrêter sur l'un des textes qui ont contribué à faire ressentir l'existence de cette entité nouvellement découverte qu'était la foule. Aux classiques ordinairement cités, Gustave Le Bon et derrière lui Gabriel Tardes, il faudrait ajouter un sociologue allemand, Georg Simmel. Je ne l'introduis pas ici pour allonger une liste déjà longue, mais parce que son point de vue, développé entre I890 et I903, tranche par rapport à celui de ses contemporains. ${ }^{6}$ Au lieu de définir un à un, et souvent de façon arbitraire, les traits spécifiques qui font de la foule une entité particulière, capable d'influer sur chacun de ses membres et de commander certaines de leurs actions, Simmel, considérant que tout rapport entre des personnes, fussent-elles deux ou cent mille, était une sociation (Vergesellschaftung), partait des individus et de leurs motivations. Comment, pourquoi des gens établissent-ils une relation? À quel type de sociation chaque occasion de rencontre donne-t-elle naissance? Simmel, un Berlinois très attentif à l'évolution rapide que connaissait alors la capitale, se posait ainsi des questions proches de celles qui inquiétaient les exploitants de cinémas en train d'ouvrir leurs salles sur le Kurfürstendam, c'est-à-dire non pas: Qu'est-ce que le

4. Das Welttheater, 3 février I9I2.

5. G. Le Bon, La Psychologie des foules, Paris, Flammarion, I895; G. Tarde, «Foules et sectes du point de vue criminel ", Revue des deux mondes, vol. 49, n II, novembre I893.

6. G. Simmel, Über soziale Differenzierung. Soziologische und psychologische Untersuchungen, Leipzig, I890; «Die Großtädte und das Geistleben» [I9O3], Grundfragen der Soziologie, Berlin-Leipzig, I9I7. 
cinéma? mais: Qu'est-ce qui peut inciter les passants à entrer? Comment une salle de spectacle devient-elle le lieu d'une sociation?

Les réponses à cette interrogation ont varié énormément au long du $\mathrm{XX}^{\mathrm{e}}$ siècle. Je ne m'arrête ici qu'aux premières décennies durant lesquelles la notion de foule s'est transformée d'objet à définir en évidence parfaitement attestée. Dans les débuts du spectacle cinématographique, les programmes étaient constitués de plusieurs films très brefs, projetés en ordre aléatoire. Quelques bandes ont été tout de suite des fictions, mais la grande majorité des vues étaient des photographies animées, prises sur les avenues et au milieu des places. Le public entrait dans la salle pour regarder ce qui se passait dans les rues - dans certaines rues. Ces petites scènes, tournées essentiellement en milieu urbain, soit dans les métropoles européennes, soit dans des cités méditerranéennes ou asiatiques, nous informent non sur la notion théorique de foule, alors en voie de consolidation, mais sur la manière dont on pouvait se représenter une foule.

La première chose qui frappe, lorsqu'on revoit ces documents, est l'absence de ce qui, pour nous, aujourd'hui, est la foule citadine. Qu'il s'agisse de Lyon, de Paris, de Turin, de Londres ou de Berlin, nous apercevons d'assez rares voitures et un petit nombre de passants. Pas de longues files pressées, pas de hâte à traverser les rues, pas de groupes qui se rencontrent, se bousculent, doivent piétiner sur place. Arrêtons-nous à l'un de ces films, Milano pittoresca, une de ces promenades urbaines que proposait, en I9I3, la Cooperativa Panoramici Italiani. Les corps des piétons qui circulent au milieu de la chaussée ou bavardent sur les trottoirs nous semblent épais. Ces deux hommes vêtus de noir, au premier plan, ont du ventre et ils ne sont pas les seuls. Les poings aux hanches, les chapeaux agités et les sourires, les larges saluts, la lenteur des mouvements laissent une impression de totale détente. Un mouvement de tête, un panama qu'on soulève instaurent une brève relation. Avec qui ? Peutêtre avec personne en particulier, avec cette agrégation fluide qui, dans sa lente déambulation, fait sienne la rue. Un camion s'approche. Sans précipitation les groupes s'écartent, deux lignes denses, au coude à coude, se forment de chaque côté: on jurerait qu'elles canalisent le véhicule et le contraignent à s'éloigner. La scène, si vivante soit-elle, ne laisse percevoir aucune impatience; l'opérateur n'a pas eu de mal à obtenir des vues parfaitement nettes. Les promeneurs s'arrêtent et, les jambes largement écartées, solidement ancrés au sol, ils adressent quelques mots à d'autres passants. Par sa manière de s'approprier l'espace, de l'occuper et le défendre, cette foule manifeste la connivence qui lui permet d'exister. 
Simmel note que moins une sociation s'attache à un but défini, plus chacun de ses membres exprime sa particularité. Comparées à une église, où le lien interpersonnel est puissant et la règle contraignante, une salle de spectacle, une place, une avenue laissent se manifester une forte individuation. La nonchalance des Milanais, leur comportement mais aussi leur apparence physique, l'indolence qu'ils affichent et qu'exprime parfaitement leur corps révèlent le plaisir que leur procure une familiarité de voisinage sans véritable implication. Elle éclaire le sentiment d'indépendance personnelle que le cinéma met en scène dans un espace urbain de faible densité, comme celui de Berlin ou de Milan, et qu'il renforce par la fréquentation de ce lieu ouvert à tous, très faiblement ritualisé qu'est une salle de projection.

Les vues que nous propose le premier cinéma ont pour cadre des espaces largement ouverts. La gestuelle, le comportement des personnes filmées manifestent une grande liberté d'allure; les corps, détendus, se font les agents de relations désinvoltes et joyeuses. On doit, évidemment, prendre en compte les impératifs techniques: l'absence d'éclairage obligeait à filmer en plein air et les promenades, rencontres, flâneries, sorties de messes, de spectacles ou de courses à l'hippodrome offraient des spectacles vivants et nonchalants, dont on tirait sans mal d'excellentes images. $\mathrm{Ni}$ le besoin de lumière ni le désir de travailler vite aux moindres frais ne justifient cependant l'attention prêtée à la promenade urbaine; on devine ici l'influence d'un véritable parti pris - assumé ou non? il est difficile de le savoir. La référence à Charles Baudelaire, derrière lui à Walter Benjamin, est un lieu commun des études filmiques: les badauds, les marcheurs oisifs auraient trouvé au cinéma un prolongement de leurs promenades. L'explication nous fait tourner en rond; admettons que les flâneurs aient eu plaisir à se retrouver sur l'écran, qu'en était-il des autres, de ceux que dix heures de travail par jour empêchaient d'arpenter les boulevards, mais qui, le soir, allaient cependant voir un film ? Le cinéma, à cette date, ne les avait pas encore rencontrés: son horizon était celui de la détente et du loisir.

\section{Corps alignés}

La guerre mondiale modifia radicalement la politique des corps; elle imposa trois images qui marquent encore notre époque, celles des corps assujettis, des corps vaincus, des corps mutilés.

Les habits changeaient d'un pays à l'autre, mais, au jour de la mobilisation, en août 19I4, les corps filmés se révélèrent partout identiques. 
Ils étaient lourds. Les hommes, alors, n'étaient pas sveltes; la fiction est obligée de tricher quand elle veut mettre en scène des soldats partant pour le front: elle les représente minces, élancés, quand ils devraient être courtauds et trapus. Nous retrouvons, maintenant sous l'uniforme, les silhouettes pesantes que nous avions aperçues au milieu des places et sur les boulevards des grandes villes; ces bersaglieri un peu trop ventrus qui se préparent à monter dans un train sont peut-être nos deux promeneurs milanais, souriants encore, pliés déjà à l'ordre militaire. Une compagnie arrive, au pas cadencé. Son mouvement est parfaitement synchrone, mais la différence est saisissante par rapport au jeu «naturel » des acteurs, jeu en fait artificiellement réglé, souple, où s'extériorise une subtile correspondance des gestes. La marche de chacun de nos militaires ne concorde pas avec la marche de ses camarades : le rythme des pas, hier libre, est devenu instinctif, il n'est même pas nécessaire qu'une voix le dirige. En quelques heures, les corps se sont transformés en machines.

Avec les hostilités, le cinéma découvre l'homme machinal, il ne l'oubliera pas. Les films de rue, les «symphonies urbaines", multipliées entre les deux guerres, font la part belle à cette mécanisation. Les lieux publics, dans Menschen am Sontag, sont traversés de courants automatisés. Qu'ils sortent du travail, qu'ils aillent à la gare, le dimanche, pour s'évader vers le bord d'un lac, qu'ils se pressent, le lundi, pour ne pas être en retard, tous les Berlinois se meuvent en mesure, les enjambées suivent les enjambées, comme si la moindre discordance risquait de paralyser la circulation. Dans The Lord of the Rings I (Ralph Bakshi, 1978), dix mille Orques affrontent dix mille Gondurians et les personnages virtuels, dupliqués deux par deux, ont été conditionnés pour que, durant le combat, leurs actions se répondent parfaitement. Le clin d'œil, amusant, n'est pas totalement gratuit: depuis près d'un siècle les corps bien réels des individus se sont, dans l'univers discipliné du capitalisme industriel, pliés à des lois identiques.

Les régimes totalitaires ont misé sur l'alignement des corps et sur leur mouvement programmé pour créer d'étonnants spectacles, mais ils n'ont fait qu'adapter à leur profit ce que le cinéma avait découvert, cette étourdissante puissance des masses en mouvement. Dziga Vertov et, davantage encore, Walter Ruttmann ont su très tôt la mettre en évidence. Berlin, Symphonie einer Großstadt, film monté, en 1927, par Ruttmann, est entièrement construit sur une eurythmie mécanique qui part des moyens de transport mais s'étend rapidement aux femmes et aux hommes. Les foules du matin sont celles, soumises et empressées, de Menschen am Sontag. Dans le train qui les porte vers la ville, les silhouettes, secouées 
rythmiquement, se penchent en avant, reculent, s'inclinent de nouveau à l'unisson. Puis, à l'usine, au bureau, dans les magasins, bras, jambes, dos, épaules s'affairent. Il ne peut y avoir de protagonistes parce qu'il n'y a qu'un seul personnage, le corps collectif.

Davantage que les documentaires, où le tourbillon des silhouettes sert souvent de prétexte à de simples variations esthétiques, les actualités dévoilent ingénument la corporéité de profils anonymes. Je pense en particulier à un numéro de Bavaria, une Wochenschau allemande très populaire qui fut diffusée jusqu'au début de la seconde guerre mondiale. Cette livraison d'août 1938 mérite l'attention parce que, dans un dessein affiché de propagande, elle oppose les vacances sportives et laborieuses des jeunes Allemandes aux futiles préoccupations des Américaines qui se présentent à des concours de beauté. Le contraste repose entièrement sur deux manières d'utiliser les corps. Les Allemandes, au matin, plongent dans la rivière, s'ébattent librement, puis se figent autour du drapeau, avant de se mettre collectivement, en mesure, au service d'une fermière. Les Américaines, alignées, calibrées, passées à la toise, sont d'emblée transformées en automates. Aux profils affranchis on oppose les profils asservis, mais il s'agit toujours de formes anonymes qu'une discipline consentie ajuste les unes aux autres.

Les films de Leni Riefenstahl ne représentent qu'un cas, spécialement brillant, parmi d'innombrables exhibitions anatomiques. La robotisation des jeunes Allemands, au deuxième puis au quatrième jour du rallye de Nuremberg, est l'aspect le plus impressionnant de Triumph des Willens (1935), celui qui a diffusé à travers le monde une représentation confondante - et fantasmatique - de la discipline nazie, tandis que Fest der Schonheit, seconde partie d'Olympia (1938), se présente en festival de la plastique masculine. Les vues du plongeoir sont à juste titre célèbres: un montage rapide saisit sous tous les angles concevables, en dessus, en dessous, de profil, les gymnastes qui grimpent, s'étirent, sautent, atteignent l'eau. Muscles tendus, cuisses puissantes, torses élancés, brasses énergiques se succèdent sans interruption - et sans mention des classements ni des médailles puisque seul compte le déploiement de formes athlétiques.

C'est ici le cinéma qui produit les corps. Riefenstahl a été l'une des ordonnatrices du congrès de Nuremberg, elle a imposé des figures, obligé les organisateurs à tenir compte du placement de ses caméras, fait répéter entièrement le cérémonial à l'avance pour pouvoir le filmer. Aux Jeux olympiques de I936, elle a soumis les installations aux nécessités des prises de vues et d'avance installé ses nombreux opérateurs en fonction des 
images qu'elle entendait utiliser. Le cinéma n'ignorait pas les Olympiades: dès 1908 il avait rendu compte de Jeux de Londres, mais il l'avait fait de manière sobre, en s'attachant exclusivement aux principales épreuves et au palmarès final, sans prêter une attention particulière à l'anatomie des sportifs. À cette logique informative, les réalisateurs des années 1930 substituèrent un imaginaire esthétisant; ils se servirent des corps, bien entendu, mais par et pour le cinéma. Un film d'actualité italien, réalisé à l'occasion de la visite de Hitler à Venise, en I938, met en lumière cette stratégie. Les officiels, placés sur une estrade, n'aperçoivent qu'une très faible partie de la démonstration organisée en leur honneur - au point que très vite ils se mettent à bavarder entre eux. La parade n'a de sens que par rapport au film qui en sera tiré; c'est à l'écran qu'elle devra être regardée.

Une quinzaine d'années séparent les foules détendues et ondulantes, filmées avant 19I4, de Berlin, Symphonie einer Großstadt. Le monde occidental avait-il aussi radicalement changé en peu de temps? Ou bien le cinéma s'était-il fixé d'autres horizons? En mars I895, voulant tester un appareil à prise de vues multiple, Louis Lumière «filma» les ouvriers quittant son usine. Il choisit un cadrage, définit une action et la fit exécuter, c'est-à-dire que, en trois prises successives, il réalisa quelques-uns des premiers films. Nous avons souvent eu l'occasion de revoir ces très modestes bandes: en une minute, une soixantaine de personnes franchissent un portail et se dispersent. Il y a tant de monde à la fois, tant de physionomies insaisissables dans leur envol, que l'impression d'anonymat domine. Ces gens n'ont qu'une identité collective, ce sont «les ouvriers des usines Lumière ", ils jaillissent d'un même endroit, unis par les heures d'atelier, par leur vêtement, par la course vers leur domicile, ils participent de la civilisation industrielle mécanique, mais ils vivent trop sur les marges urbaines pour qu'on s'attarde à les filmer.

Le premier cinéma nous laisse très rarement entrevoir des corps mobilisés. En juillet I896 le représentant toulousain des Lumière se rendit à Carmaux pour réaliser, autour des fours à coke, quelques images spectaculaires. L'une des prises, Chargement $d u$ coke, est historiquement le premier document filmique sur l'exploitation de la main-d'œuvre féminine. À cette date, on n'avait pas encore vu de cinéaste à Carmaux, et cent exemples nous montrent que les personnes filmées, même quand on leur demandait de feindre l'indifférence, ne pouvaient s'empêcher de jeter un regard vers la caméra. Ici au contraire les femmes ne songent pas à lever la tête, elles sont comme rivées à leur tâche. Les chapeaux de paille qui cachent leurs visages, de lourdes robes, sans doute trop chaudes pour 
une journée d'été, les rendent absolument anonymes. Elles devraient, avec des fourches, charger la houille dans des brouettes, pour la porter ensuite vers le four mais, voulant aller vite, elles préfêrent travailler à mains nues. Mécaniquement elles se baissent, ramassent, jettent les morceaux de charbon dans la charrette, se penchent à nouveau, partent enfin, comme tirées en avant par la charge. Une minute de film suffit pour deviner l'immense fatigue qu'engendre cette réduction des corps à l'état de machine.

Au cours des mêmes années, d'autres films montraient l'exceptionnel, l'exotique, les villes d'Asie, les bords du Gange, ceux du Nil, où des silhouettes enchevêtrées, agitées, maigres, indistinctes, cachées aussitôt qu'aperçues, semblaient littéralement grouiller. Ces vues avaient en commun le trop-plein, l'écran ne parvenait pas à contenir une houle fluctuante qui lui échappait par tous ses bords. La foule, ici, représentait l'ailleurs, l'univers inquiétant des trop grandes villes, les troubles, la densité de rassemblements massifs où les corps risquent d'être écrasés. Indirectement le cinéma désignait une menace que dénonçaient certains penseurs politiques. Il n'est pas surprenant que des Français, et particulièrement Le Bon, obsédés par le souvenir des «journées révolutionnaires", d’octobre 1789 à mai I87I, aient mis l'accent sur les dangers que représentaient les corps anonymes, tandis que Simmel, indifférent à un passé qui n'était pas le sien, mais sensible à ce qui se passait dans les rues ou dans les cinémas, cherchait à comprendre comment des individus s'associaient tout en restant indépendants.

La mobilisation des corps était effective bien avant que ne débute le premier conflit mondial, mais le cinéma lui accordait peu d'attention. Pourquoi se l'est-il appropriée dans les décennies suivantes? Avant les hostilités, les films montraient parfois une foule immense et inconstante, beaucoup plus fréquemment des personnes réunies par un lien extrêmement léger; ils illustraient ainsi le point de vue de Simmel, ignoraient celui de Le Bon. Après la guerre, les analyses de Simmel furent à peu près oubliées: il est symptomatique que Freud, dans son étude de la psychologie collective, se réfère exclusivement à Le Bon et ne cite jamais le sociologue berlinois. La guerre, impliquant toutes les classes sociales, avait banalisé l'enrégimentement. Aux tranquilles déambulations du début de siècle, elle avait substitué le groupe compact où s'absorbaient les individus. Une bande d'actualité de la Gaumont British nous présente, en I935, les Blackshirts d'Oswald Mosley défilant dans Londres: spontanément ils se sont mis en rangs et marchent en cadence. Les cinéastes ont alors, en de nombreuses circonstances, filmé des masses roulantes: infi- 
nités de têtes tournées vers un orateur, foules piétinantes, avançant et reculant d'une seule poussée, roulant au travers des rues, s'écrasant aux carrefours. Les passants filmés vers I9I3 avaient une physionomie; ceux de 1930 étaient indistincts, leur visage, ignoré, comptait moins que leur silhouette, la caméra, suivant leurs pas, croisait d'autres mouvements et quadrillait l'espace de trajets anonymes. Le cinéma documentaire avait changé le statut des corps.

\section{Corps sacrifiés}

Les régiments ont peuplé l'écran aux premiers jours du conflit, puis ils ont cessé de parader: la caméra, dans les étroites tranchées, n'aurait saisi que quelques hommes et d'ailleurs montrer l'air grave des troupes montant au front, ou l'épuisement de celles qui en descendaient, aurait sapé le moral de l'arrière. La masse humaine n'a cependant pas disparu, elle a simplement changé d'identité. Pendant quatre ans, les longs défilés ont été essentiellement ceux de prisonniers. Leurs interminables colonnes, en ligne de deux ou de trois, leurs arrêts, leurs repas, leur installation entre des barbelés n'ont pas eu comme seul objectif de remplir les programmes: le corps du captif est aussi, par antiphrase, un hommage à la valeur guerrière de ceux qui l'ont pris. L'homme est désarmé, parfois tête nue, inquiet, affecté par sa reddition, secrètement content d'échapper au combat; il a perdu la raideur qu'il affectait au cours des prises d'armes; sa silhouette flottante, ni civile ni belliqueuse, la fatigue contre laquelle il ne cherche plus à lutter, révèlent les faiblesses de l'adversaire.

Les prisonniers encombrent; il faut les nourrir, les garder, on ne sait pas quoi en faire. Ils sont aussi un symbole : à travers la manière dont il les traite, un pays peut prouver au monde extérieur sa magnanimité et, qui sait, convaincre quelques adversaires de déserter. Partout on a filmé des soins aux captifs et de généreuses distributions de vivres. Les AustroHongrois se sont montrés particulièrement attentifs à cet égard: il leur fallait rassurer les minorités slave et italienne sur le sort réservé aux prisonniers russes ou italiens. On trouve ainsi, dans les cinémathèques de Vienne et de Budapest, des documentaires d'une excellente qualité sur les camps de captivité. Première étape, l'hygiène : on fait déshabiller les hommes, on désinfecte leurs vêtements, on les fait se laver - des précautions certainement indispensables qui, à l'écran, font voir des silhouettes nues, privées de ce semblant d'identité qu'était l'uniforme. Ensuite, les baraquements, 
les rassemblements, les appels, une gestion minutieuse de la détention. Revus aujourd'hui, ces documentaires qui avaient pour but d'humaniser la guerre annoncent l'enchaînement qui, partant de la mobilisation, conduisit à l'internement, puis à l'anéantissement de millions de corps.

L'ardeur avec laquelle le cinéma, dans l'entre-deux-guerres, représenta le mouvement et la perfection des corps athlétiques n'était-elle pas une réaction contre l'asservissement et la destruction provoqués par la guerre? À côté de l'enrégimentement et de l'emprisonnement, le conflit a laissé une troisième image que le monde, alors, refusa de voir, qu'il a toujours du mal à regarder, celle de la mutilation. Les actualités, dans les années 1920, montrèrent ce qui était acceptable parce qu'immédiatement présent, quotidiennement visible dans les lieux publics: des bras ou des jambes coupés, des mains sans doigts qui tentaient d'apprendre à écrire, des invalides poussés, sur leurs petites voitures, par des infirmières, des amputés cherchant, avec une lente maladresse, à se servir de leurs prothèses, des cannes blanches, des veuves tout en noir qu'on faisait défiler, à la place de leurs maris, dans les cérémonies officielles. Ultérieurement, pour des émissions historiques, on alla jusqu'à exposer des monceaux de cadavres enlisés dans la boue, des morts placés dans des cercueils en sapin, des blessés sur leurs brancards. Mais l'inacceptable était, est toujours, l'atteinte au visage qui nie le corps, le rend méconnaissable, inhumain. Les médecins du Val-de-Grâce, ceux de l'hôpital Rizzoli, à Bologne, ont récolté une abondante documentation consultable seulement sur demande expresse. Yeux crevés qu'on doit extraire de leur orbite pour qu'ils ne pourrissent pas, faces aplaties, sans yeux, sans nez, plages lisses de chair reconstituée, mâchoires arrachées, trou noir où ne restent ni langue ni dents. Archivée, cette misère sans espoir demeure cachée; on la supporte quand elle est racontée, l'écrit, la parole ménagent une distanciation suffisante, mais l'image, elle, est insoutenable.

À partir de la guerre, les corps sacrifiés - mais jamais les visages deviennent un accessoire de propagande, un outil pour susciter la pitié ou provoquer la haine. Dans une situation de grande faiblesse, l'exposition des victimes peut réveiller la commisération des spectateurs, les amener à faire un geste. Pendant la grande famine qui frappe la Russie en I92I, les Soviétiques n'hésitent pas à envoyer au reste du monde des films où apparaissent des bandes d'enfants en guenilles, des cadavres exsangues, des squelettes vivants fouillant les poubelles: la déchéance des corps servit à lancer un appel au secours. La République espagnole recourut au même procédé pendant la guerre civile. Son aviation étant incapable d'arrêter les escadrilles allemandes et italiennes, elle prit prétexte de la panique, des ruines, des blessés pour 
réveiller l'opinion dans les démocraties. Au bruit des sirènes des femmes et des enfants affolés courent vers les abris, se bousculent, certains tombent. Sous l'effet de la peur, les corps se délitent, les silhouettes mécanisées, qui, quelques années auparavant, caractérisaient la foule urbaine, sont abolies et, dans un sauve-qui-peut désordonné, les individus se transforment en ombres mouvantes. Sur le front même, les républicains affichent leurs pertes; brancards surchargés, membres et têtes bandés, salles d'hôpital évoquent des combats où le peuple oppose des poitrines à des chars d'assaut.

Pendant la première guerre mondiale, aucun des deux camps n'avait osé montrer les énormes masses d'hommes enterrées dans les tranchées ou engagées dans les batailles. À cet égard, le conflit espagnol marqua une rupture. Face à la détresse républicaine, les nationalistes construisirent, en images, des assauts fantastiques où l'élan des muscles emportait tout; leur armée n'affichait ni obstacles ni pertes et, victorieuse, elle défilait, compacte, rigide, dans les villes conquises où le chœur des habitants, bras levés, lui faisait une haie d'honneur. Conquérantes, les dictatures de droite offrirent le spectacle d'offensives sans larmes. L'horrible expression de «frappes chirurgicales» n'existait pas encore, elle caractérise cependant très bien la vision des hostilités que l'Allemagne proposa à ses citoyens et au monde à partir de 1939. Elle fit voir l'avance continue, face à un adversaire peu visible, d'unités au sens propre, in-corporées, soudées en un seul corps. D'athlétiques jeunes gens au coude à coude traversaient des ruines, croisaient des armes ou des véhicules abandonnés par l'ennemi, ne rencontraient jamais de cadavres. À la fin de I94I la Wehrmacht découvrit les restes de vingt mille officiers polonais massacrés par les Soviétiques. Elle tourna un film, Im Wald von Katyn, sinistre étalage de victimes propre à ternir la réputation de Moscou, mais elle ne le diffusa pas, comme s'il fallait à tout prix perpétuer le mythe de la guerre propre. Les Soviétiques, au contraire, ne perdirent pas une occasion de souligner la dé-corporation infligée à l'envahisseur : des Allemands réduits en miettes, étripés, congelés par le froid remplirent les actualités; les dizaines de milliers de soldats pris à Stalingrad défilèrent interminablement sur les écrans, la population ne se lassa pas d'observer leur fatigue, leurs bandages, leurs vêtements inadaptés au climat. Tant qu'elle avait été la plus forte, la Wehrmacht s'était acharnée sur les civils auxquels le cinéma offrait une étrange revanche : la débâcle des tortionnaires rendue évidente par sa déchéance. Rencontrées lors d'un passage à travers une ville ou dans la campagne, les colonnes de captifs n'auraient pas produit le même effet: la mise en images conférait une dimension épique à la reddition de l'adversaire. 
La vengeance par l'image est devenue une arme. Après Diên Biên Phu les longues files de prisonniers français ont été filmées de face, du haut d'un portique, les silhouettes amaigries, épuisées, démoralisées ont été envoyées aux peuples colonisés pour leur annoncer la fin de la domination blanche. L'anéantissement physique d'un ennemi a cessé d'être une opération nécessaire mais malpropre qu'on mène sans tapage : il devient utile de l'enregistrer et de le rendre public. Durant la «purification ethnique» en Bosnie une unité de «Scorpions» serbes a trouvé normal de filmer les humiliations et les tortures qu'elle infligeait à des musulmans avant de les exécuter; la dégradation de leurs corps justifiait la liquidation de ces hommes, puisqu'ils avaient d'abord été réduits à cette condition abjecte.

\section{Corps à la peine}

Les images de guerre posent la question de la corporéité dans le monde où nous vivons, elles illustrent la réflexion de Primo Levi: ce n'est que cela un homme, de la chair qu'on peut robotiser, déformer et anéantir. À cet égard, l'âge atomique n'a rien inventé; il a, au pire, perfectionné les moyens de faire disparaitre le corps autre, insupportable, le «corps étranger». Le cinéma a tenu un rôle ici : il a mis en évidence une suite de transformations qu'il a rendues manifestes en les exposant. La première guerre mondiale a bouleversé l'atmosphère urbaine; le va-et-vient des troupes, les convois ont multiplié les déplacements, accéléré les échanges et donné un essor inattendu à la circulation automobile. Dans le même temps, les opérateurs, qui n'avaient pas accès aux zones de combat, se sont rabattus sur ce qu'on leur laissait filmer: l'agitation provoquée à l'arrière par le conflit. Le trafic, dans certaines rues, était sans doute plus dense en I9I6 qu'il ne l'avait été une décennie auparavant, mais était-ce à un point tel que le cinéma se trouvait obligé d'en rendre compte? C'est toute l'ambiguiité du documentaire qui entretient toujours un rapport incertain avec ce qu'il entend représenter. En observant les comptes-rendus filmiques d'événements sportifs ou de courses hippiques, on note que, après la guerre, le regard des cameramen s'est porté toujours plus vers les tribunes, aux dépens parfois de la compétition. Était-ce que les spectateurs avaient changé, qu'une clientèle nouvelle était apparue? Que le public des cinémas avait d'autres exigences? Que les opérateurs n'étaient pas sensibles aux mêmes situations que leurs prédécesseurs d'avant-guerre?

Dans la seconde moitié du $\mathrm{XX}^{\mathrm{c}}$ siècle, la télévision a imposé des façons 
différentes de travailler; les dimensions de son écran, le caractère domestique de son usage, l'obligation de diffuser en permanence l'ont conduite à privilégier les entretiens ou les jeux opposant de petits groupes de concurrents, et à mettre en évidence la tête des intervenants. Les documentaires cinématographiques, dont la diffusion dépend des chaînes télévisuelles, ont fait leur cette manière de filmer, les interviews de personnes identifiées, prises en gros plan, y tenant une place croissante; les lois de la concurrence et la faiblesse des budgets rendent compte d'une évolution qui ne répond à aucun choix stylistique. J'ai ouvert cette parenthèse pour rappeler combien il est difficile d'expliquer les changements affectant la visée cinématographique. Que filme-t-on, et pourquoi? Bornons-nous à le constater, les cinéastes, après le premier conflit mondial, se sont intéressés aux mouvements collectifs qu'ils sont allés chercher dans les rues passantes. Leur caméra, elle-même mobile, ne s'arrêtait sur personne en particulier. Parcourant l'espace, multipliant les points de vue, elle donnait à voir des foules anonymes, compactes et instables, c'est-à-dire des profils relativement interchangeables et privés de physionomie.

Le corps sans nom a caractérisé le documentaire filmique jusqu'aux dernières décennies du $\mathrm{Xx}^{\mathrm{c}}$ siècle. L'absence de visages reconnaissables - à moins qu'ils ne soient pathétiques comme ceux des demeurés gâteux filmés par Luis Buñuel pour Las Hurdes (1932) ou des «monstres» utilisés par Tod Browning dans Freaks (1932): à nouveau des corps souffrants fut une particularité du film informatif qui le distingua nettement des fictions. À cela s'ajoutait une seconde différence, inséparable de la première: ces corps privés de figures se manifestaient par leurs mains. Berlin, Symphonie einer Großstadt est un ballet de doigts qui prennent des instruments, tirent des manettes, frappent des claviers, attachent, détachent, saisissent, déplacent, rangent et dérangent; un profil s'esquisse parfois, vite un recadrage nous ramène aux poignets, à l'outil premier avec lequel on entame n'importe quelle besogne. L'exemple a été retenu parce que cette œuvre est universellement connue. On aurait pu en prendre cent autres, ainsi N.U., Nettezza urbana, documentaire signé par Michelangelo Antonioni (I943) et consacré aux éboueurs de Milan qui, la tête dissimulée par une casquette, plongent les bras dans les ordures. Et aussi tel film sur un atelier de paillage où les yeux des femmes nous sont cachés quand le jeu de leurs mains est bien apparent (Dalle paglie alle maglie, Luisa Cigognetti, 1989), ou encore ce marché en plein air, têtes penchées et insaisissables, paumes actives (Belleville 94, collectif Vincennes, 1994). L'investissement corporel est la trame de La Lotta dell'uomo per la sua sopravvivenza, vaste 
fresque à laquelle Roberto Rossellini se consacra de 1967 à 1969: les mains y saisissent la matière inerte, prennent les plantes, le minerai, le fil et les transforment. Ce film n'avait pas besoin de commentaires, de nombreux passages y sont «muets»; il suffit de voir les doigts tisser, modeler la cire, traire les animaux, pour deviner qu'ils «pensent» en s'activant. Le corps, dans le film informatif, n'est pas celui d'une personne identifiée, prise dans une relation avec une autre personne, il est une paire de jambes qui court vers le bureau ou vers l'usine, une paire de bras attelés à un travail.

Des pieds et des mains mobilisés pour l'effort, de longues enjambées pour monter dans les trains, des bousculades au milieu de la rue, des athlètes et des hommes de peine musclés, des soldats qu'on fait défiler ou charger l'ennemi, des cadavres abandonnés: le corps à la peine est la matière première du documentaire. Balász vantait l'éloquence, la chaleur des visages en gros plan qui, assurait-il, dévoilent les individus et font comprendre leur destin. ${ }^{7}$ Une main, des épaules, un élan de la personne sont-ils beaucoup moins parlants? Ce qui est en cause, ici, est-il bien l'expressivité? Ou s'agit-il de tout autre chose, du balancement entre la peine physique et les sentiments, entre le travail et le répit? Le documentaire s'attache aux corps actifs; la fiction, aux visages, à leurs inflexions, à leurs échanges. Les héros, dans les films du répertoire, surmontent d'impressionnants obstacles, meurent parfois salement, mais ne s'astreignent à aucune routine; les scénarios sont lourds de chagrins, de blessures, de larmes, rarement de tâches matérielles et banalement manuelles; ils opèrent une trans-figuration, un report sur la figure qui laisse de côté les pesanteurs corporelles. Les acteurs - et le montage - font oublier les obstacles que le monde oppose aux humains; les personnages de la fiction ne tâtonnent jamais et leurs gestes limpides, leurs déplacements évidents se jouent des objets et de l'espace. Si l'on cherchait à confronter documentaires et fictions, le partage se ferait peut-être autour du corps, précisément d'une scission du corps entre sa part la plus malléable, la plus menacée, la plus ouvrière, et sa part spirituelle, sentimentale, intangible.

\section{Corps détourés}

Cependant, opposer la fiction au documentaire est, du point de vue où nous nous plaçons ici, un raccourci trop facile. Les films informatifs ne

7. B. Balász, Der sichtbare Mensch oder die Kultur des Films, ouvr. cité, p. 64. 
sont pas tous construits sur l'anonymat d'une foule mouvante, le Nanook de Robert Flaherty, celui de Nanook of the North, est un véritable personnage. Certes, ses traits apparaissent peu à l'écran, au contraire de ses mains qui construisent, réparent, creusent et tuent, mais le documentaire l'isole, le met en valeur, en fait le héros d'une épopée du Grand Nord.

Inversement la masse humaine ne sert pas uniquement à remplir le fond du décor. Sans avoir de rôle à proprement parler, elle assure, par son mouvement et par sa présence physique, une fonction contrastive. La séquence de la ville, telle que Friedrich Murnau l'a conçue pour Sunrise (I927), serait une banale confrontation psychologique si la réconciliation des époux, après la tentative de meurtre, ne se déroulait pas au milieu des passants et ne juxtaposait pas leurs deux silhouettes immobiles à une agitation urbaine frénétique. À sa descente du tram, la jeune femme qui veut fuir est sauvée d'une collision par le mari, leurs corps raidis dessinent au milieu de l'écran un bloc inerte tandis que les promeneurs, nettement séparés d'eux, s'écoulent à l'arrière du champ. Après un bref passage dans un café, simple entrée et sortie, ils disparaissent, d'abord éclipsés par les passants, puis réfugiés dans un coin d'immeuble, exilés de la vie qui, elle, suit son cours. Ils renouent enfin et sont alors pris dans le tumulte de la rue, encerclés par elle. Le passage ne justifie pas le revirement d'Indre que son mari voulait tuer, qui se sent terrorisée par lui, et cependant finit par l'embrasser avec passion. Sans doute un tel revirement échappe-t-il à toute tentative d'explication, et la rentrée dans le groupe est-elle le meilleur moyen de l'exposer sans l'interpréter.

L'originalité du film tient à ce qu'il suggère habilement, à travers le contraste des formes, d'abord la sidération (les jeunes gens paralysés, exclus), puis le retour à soi et au monde; le croisement des silhouettes rend superflu tout dialogue. Sunrise, cependant, reste une ouvre traditionnelle, centrée sur un classique trio amoureux; le film, si l'on met à part deux séquences, celle de la ville et le suspens final, est dominé par les plans rapprochés de visages. La masse humaine offre un contrepoint facile pour mettre à part et même individualiser les personnages principaux. Dans une œuvre aussi peu narrative que Desistfilm, l'un des premiers films de Stanley Brakhage, le seul fait d'isoler un jeune homme au sein d'un groupe, de le faire chahuter puis chasser par les autres, confère au banni une personnalité; l'agitation de cette petite troupe crée un corps particulier en l'extrayant d'une confusion de gestes et de membres au sein de laquelle s'est plongée la caméra. Le groupe éclipse le corps du protagoniste s'il parvient à l'attirer, l'encercler, le retenir. En revanche la foule 
construit le personnage dès lors qu'elle l'expulse. King Vidor illustre la première option avec The Crowd (1928). Si to beat the crowd est l'idéal que s'est fixé le personnage central, John Sims, le film montre en permanence un individu à la lisière de l'absorption; dans son excursion à Coney Island, dans sa recherche d'un travail, dans ses démarches quand il vend des aspirateurs, il existe seulement parce que l'image le tire de la cohue, sa silhouette s'affirme par retranchement. Mais, à terme, la masse informe triomphe et, dans l'étonnant plan final, vertigineux travelling arrière, le «héros» est annihilé, siphonné (aspiré) par l'informe pêle-mêle.

Dans Fury (1936), en revanche, Fritz Lang construit son protagoniste, au départ assez insignifiante figure, en réplique à l'éclosion de la foule. Les passants que croise Joe Wheeler, à son arrivée dans la bourgade où vit sa fiancée, sont des individus distincts. Mais quand, s'imaginant qu'il a enlevé un enfant, ils se ruent vers la prison où on l'a enfermé, en exigeant son lynchage, une déstabilisante contre-plongée transforme les marcheurs en un monstre polymorphe. Joe Wheeler, d'abord au cours de sa fuite, puis dans les manœuvres grâce auxquelles il envoie ses poursuivants au tribunal, acquiert une personnalité. Entre peur et désir de vengeance, son physique change, il devient un autre. Il est intéressant de noter que, dans les deux films cités, la foule se fait magma grâce à une prise de vues particulièrement sophistiquée et que la caméra «détoure » les personnages, John Sims avant sa résorption, Joe Wheeler quand il doit affronter la multitude.

Fury est un film parlant : la sonorisation nous fait oublier qu'il est à peu près contemporain de The Crowd. Les deux œuvres appartiennent au courant unanimiste qui a traversé Hollywood entre les deux guerres et dont Meet John Doe, dirigé en I94I par Franz Capra, serait la forme achevée: Gary Cooper, à l'époque, selon le Motion Picture Herald, l'une des dix stars du cinéma, y est littéralement engendré par le public dont il semble n'être qu'une émanation. Une journaliste licenciée invente un citoyen quelconque, John Doe, fantôme auquel elle se voit obligée de donner une consistance. Un autre homme sans importance, incarné par Gary Cooper, fera l'affaire, mais les lecteurs se sont pris d'affection pour John Doe, raccourci de leurs misères et de leurs frustrations; ils transforment le timide garçon en vrai héros du peuple souffrant. De manière particulièrement recherchée, la foule est mise à contribution pour arracher à un pseudoanonymat le corps de celui qui est en réalité une vedette, et lui permettre de jouer en solo son rôle de leader charismatique. 


\section{Corps déliés}

Détourer le corps est une ruse habile grâce à laquelle deux ou trois acteurs sont le film, le font exister par leur connivence, en laissant croire au spectateur qu'ils proviennent, comme lui, de l'anonymat. Le cinéma, cependant, a très tôt exploité un autre type de figuration en jetant sur la scène des troupes gigantesques de figurants qui ne servaient pas de faire-valoir à un artiste, qui n'étaient reliés entre eux par aucun projet et ne constituaient rien d'autre qu'une masse turbulente, une foule au sens le plus large du terme. La fiction a connu elle aussi la turbulence des corps sans visages, avec des modalités différentes de ce que nous avons repéré dans les documentaires, et dans un registre qui nous éloigne sensiblement du tête-à-tête entre acteurs.

L'affaire s'est d'abord jouée entre l'Italie et les États-Unis. Arrivés tard à la production, les Italiens misèrent sur les éléments dont ils disposaient: une lumière constante tout au long de l'année, des ruines antiques et une immense quantité de main-d'œuvre à bon marché. Lorsque, en 1913, les premières reconstitutions antiques traversèrent l'Atlantique, les Américains ne cachèrent pas leur étonnement. Il y avait là une manière de travailler qu'ils n'avaient pas encore envisagée et ils reconnurent, sans hésitation, la prodigieuse habileté des Italiens «dans la disposition océanique d'un grand nombre de personnes et dans les décors parfaitement adaptés et harmonieux qui les entoure ${ }^{8}$

Les studios de la péninsule se contentaient de scénarios élémentaires et dépourvus de réelle continuité. Il leur était donc facile d'interrompre une intrigue peu consistante pour mettre en route un bataillon de comparses. La même indifférence à la logique narrative permettait toutes les audaces de montage, les coureurs déchaînés pouvaient arriver à gauche, ressortir en haut, rentrer à droite: leur trajectoire imprévisible, n'ayant pas de signification précise, n'étant rien d'autre que du pur mouvement, devenait merveilleusement libre. Les procédures techniques étaient aussi simples qu'efficaces. La caméra était placée en oblique, généralement en plongée. Une bordure de fenêtre, l'angle d'un monument, un escalier assuraient un encadrement inusité, vaguement étrange. Le monteur faisait partir le plan au moment où la foule venait de franchir le bord de

8. The Moving Picture World, I3 décembre I9I3 (nous traduisons). 
l'écran: l'intrusion était rapide, brutale, mais elle était vite interrompue pour faire place à un autre déplacement, plus proche ou plus lointain, toujours aussi inattendu et aussi bref.

Certaines prises de vues étaient d'une grande qualité plastique, mais ce qui, aujourd'hui encore, demeure impressionnant est la force étourdissante de ces extravagantes cavalcades humaines. Serrés les uns contre les autres, roulant comme une vague, les figurants déchirent l'espace. Dans Giulio Cesare (1913) l'attaque des légionnaires, prise en plongée verticale, devient le roulement fougueux, l'écoulement d'un monstrueux quadrilatère vivant. Ailleurs, dans le même film, l'avancée des troupes romaines est montrée à travers une série de plans d'ensemble; la colonne en marche occupe tour à tour des places différentes sur l'écran, sa progression est fantaisiste, absurde même si on l'envisage en termes logiques, mais ces variations arbitraires, rendues frénétiques et puissantes par la vivacité du montage, créent une incomparable sensation de force.

Les foules, dans les films unanimistes, sont étroitement reliées aux protagonistes, elles paraissent les générer, mais elles n'existent que pour les servir. Les foules des péplums italiens sont indépendantes des acteurs, elles ont leur vie propre, elles manifestent toute l'impétuosité de corps jeunes qu'on paie pour s'ébrouer et se répandre à travers le paysage; leur galopade est ludique, entrainante, immotivée et attrayante. Les stars, en jouant, ne perdent jamais de vue la représentation qu'elles donnent d'elles-mêmes à travers leur manière de se poser comme à travers leur rapport aux autres comédiens; elles adaptent leur visage, éventuellement leur corps, à l'idée qu'elles se font de leur présence à l'écran, et au-delà de leur public. Les figurants, dans les œuvres historiques, n'avaient aucune de ces préoccupations, ils n'avaient pas d'image à sauvegarder. La panique qui saisit les Romains, dans la seconde version des Gli ultimi gionri di Pompei (I9I3), illustre cette liberté de comportement. Les fuyards suivent les directions indiquées par le réalisateur en mimant ce qui, pour eux, est une manifestation de peur: on se trouve en présence de trajets coordonnés, entrecroisés, canalisés, et de gestes individuels improvisés; les silhouettes sont à la fois associées dans leur course et désaccordées dans leurs réactions. C'est là également ce qui sépare les foules du péplum des groupes figurant dans les documentaires. Nous l'avons noté, les corps des films informatifs sont encadrés et alignés, captifs de leur activité, qu'elle soit d'ordre militaire ou laborieux. Les figurants des œuvres historiques peuvent se mettrent en file ou en carré, ils n'en sont pas moins indépendants; ils travaillent sans doute, mais leur tâche est un simple divertissement. 
Si remarquables soient-elles, de telles séquences sont à peu près dépourvues de sens. Les Américains comprirent vite ce qui manquait aux films italiens et comment eux-mêmes devaient utiliser des armées de figurants. Moins de deux ans après Giulio Cesare, David Wark Griffith, avec The Birth of a Nation, introduisait à l'écran une autre manière de faire intervenir des masses humaines. Il ne saurait être question de revenir sur les bouleversements que provoqua le premier long métrage du cinéma américain; en ce qui nous concerne, ici deux mots suffisent: expressivité corporelle. La foule, dans ce film et dans Intolerance, apparaît soit pour concrétiser un événement, pour le rendre immédiatement lisible, soit pour faire naitre l'émotion. Les figurants ont un rôle essentiel dans les instants de grande tension : ils ne se bornent pas à remplir l'écran, leur présence souligne la gravité du moment. Dans The Birth of a Nation des séquences telles que la mobilisation des sudistes, le raid nordiste sur la petite ville de Piedmont, la très impressionnante reconstitution du bombardement sur Atlanta reprennent le procédé inauguré par les Italiens - un groupe compact avec des comportements individuels différents - en le chargeant de sens. Les héros du film n'interviennent pas au cours des épisodes cités, la figuration est l'événement lui-même, la gestuelle anonyme des figurants suffisant pour le créer et le rendre dramatique. Les comparses, par leur mimique et leurs démonstrations, rendent également sensibles une atmosphère, un trouble, un conflit. La violence qui marque les deux procès d'Intolerance, celui du Christ et celui du jeune homme innocent, tient à la manière dont le film regarde le public et laisse deviner ses sentiments; le véritable protagoniste, pendant les comparutions, n'est pas l'accusé qui se tient en retrait, c'est la foule dont les gestes «parlent».

\section{Corps politiques}

Après The Birth of a Nation, la force expressive que recèle une masse humaine à la fois bien dirigée et laissée relativement autonome était devenue évidente; au travail des acteurs, soumis à la double contrainte du cadre et du gros plan, se juxtaposaient le dynamisme et la présence corporelle des foules. Interrogé par un journaliste, Arthur Schnitzler lui confiait qu'il ne voyait guère d'avenir au cinéma, pratique à son avis inférieure au théâtre, mais que certains réalisateurs provoquaient son admiration, et spécialement Mihály Kertész (il deviendra Michael Curtiz en partant pour Hollywood), «par la maîtrise avec laquelle il crée des mouvements de masse 
et par son inépuisable énergie ».9 Maîtrise, énergie, les mots disent bien qu'il faut de la poigne pour dompter les foules. Célébrant les mérites du même Kertész, Balász, porte-drapeau du cinéma d'avant-garde, affirmait:

Kertész s'est révélé un grand maître des scènes de masse. Lubitsch ou Griffith nous ont offert des mouvements de masse plus impressionnants. Mais nous n'avions jamais vu des masses disposées de telle manière que tout détail y soit vivant et significatif. Il n'y a pas chez lui que l'ensemble, le moindre élément est conçu à part et revêt sa propre signification. ${ }^{10}$

Plus théoricien que Schnitzler, Balász mettait en évidence, à partir de noms propres, les trois manières possibles de transformer une foule en acteur: d'abord, avec Kertész, la combinaison du multiple et de l'individuel, dans la tradition italienne, mais avec une stricte régulation des mouvements; puis, à la suite de Griffith, l'effet narratif; enfin, comme le suggère le nom de Lubitsch, l'expression politique, que ce soit contre la révolution, ou contre l'ordre établi.

Grande Guerre, révolutions européennes, milliers d'hommes dans les tranchées puis dans les rues: il n'y a rien de surprenant à ce que la force collective, très présente dans les esprits, ait marqué le cinéma des années I920. Ernst Lubitsch sans doute mais en contrepartie Sergueï Eisenstein, ou, pour être plus précis, Madame Dubarry (I9I9) et Octobre (I927). Le premier film camoufle un violent pamphlet contre-révolutionnaire derrière les aventures sentimentales d'une favorite royale. Pour élémentaires qu'elles soient, les procédures ne manquent pas de force. Au départ, une rue calme, le rectangle immaculé d'une place vide en plein soleil. Une horde arrive, déchaînée, gesticulante; on a volontairement laissé les figurants donner libre cours à leur fantaisie; leurs contorsions, les bras et les piques qu'ils soulèvent anarchiquement, la bousculade détruisent l'harmonie du décor. Une pagaille identique accompagne l'invasion du palais royal, puis l'exécution de la Dubarry; la guillotine, démembrement du corps, rend tangible la destruction du corps social par des corps enfiévrés et incontrôlables.

Madame Dubarry est une affirmation massive et passionnée, Octobre une démonstration. Le film s'ouvre sur sept plans où se projettent, tout seuls, des faux levées et des fusils crosse en l'air: les paysans, l'armée se soulèvent, leurs symboles envahissent l'écran, pourtant leurs corps font défaut. Les scènes de répression misent sur l'opposition entre deux types de silhouettes.

9. «Aus einem Gespräch mit Arthur Schnitzler», Neue Freie Presse, 5 octobre 1923. IO. B. Balász, «Der Junge Medardu», Der Tag, 9 octobre 1923 (nous traduisons). 
L'épisode de février est célèbre à cause de son hallucinant montage: des images ultra-rapides (trois ou quatre photogrammes) d'un mitrailleur se succèdent en rafale; seul le rictus de l'homme, solidement assis derrière son arme, est discernable. En face la manifestation dispersée se réduit à des cadavres, une femme dont les cheveux épars cachent le visage et un cheval, silhouettes immobiles, silhouettes sans noms. Pour le rassemblement de juillet, le cinéaste s'est inspiré d'une photographie fameuse, prise du haut d'un toit, mais il l'a interprétée à sa guise, ses figurants sont beaucoup plus nombreux que ne l'étaient les protestataires et, au lieu de fuir, logiquement, vers le fond, ils avancent au premier plan, offrant leurs poitrines aux mitrailleuses. Au peuple il manque toujours un corps ou plus exactement un corps mobile, capable de réagir au lieu de se laisser massacrer. Un passage caractéristique montre, en alternance, des marins armés et un orateur politique; rien ne bouge, la parole, sagement écoutée, demeure sans effet.

La construction d'une entité révolutionnaire est l'objet de la seconde moitié du film. Du côté de l'ancien régime s'impose une présence, une assise physique massive attestée par la reprise de formes identiques (ainsi le mitrailleur en février). Chez les bolcheviks intervient une lente mise en place dont on observe parfaitement le mécanisme dans le passage consacré à la défaite de Kornilov, le général qui tentait de prendre le pouvoir. La séquence est intentionnellement déroutante. Les militants sont arrêtés par la police, la répression se met en place, les dépôts d'armes sont fermés. Pourtant voici une troupe armée qui avance; l'association d'ouvriers en blouses, de femmes reconnaissables à leurs jupes, de soldats ne laisse pas de doute : il s'agit de révolutionnaires. La contradiction est forte ; elle s'explique a posteriori quand, de manière insistante, dans un montage rapide mais très clair, des mains s'emparent de fusils. L'image du début est inversée, les armes ne se déplacent pas seules: le peuple les saisit parce que, cessant d'être un groupe amorphe, il est devenu un corps actif qui se constitue en classe; il est désormais le prolétariat. Dans tout le film Lénine ne fait que trois brèves apparitions. Il montre une voie, mais c'est la masse politisée qui, au pas de charge, se saisit du pouvoir.

\section{La fin des corps?}

Les corps politiques n'ont pas de visage. L'affirmation risque de surprendre, je la précise en opposant le corps politique au personnage politique. De Lénine en octobre à Mussolini dernier acte, de Rosa Luxembourg 
à JFK et Nixon, le cinéma a largement mis en scène les grandes figures de l'histoire. Pour y parvenir, des acteurs se sont donné une allure compatible avec l'image peu précise que le public s'était forgée de la personne dont on leur retrace l'existence, et la conviction dont ils ont su faire preuve, qu'ils ont imprimée sur leurs visages, a rendu vraisemblable une très approximative ressemblance. Une silhouette d'acteur, familière à force de revenir sur l'écran, suffit pour rendre crédible le portrait d'un leader assez vaguement connu du public. Si le personnage exprime des opinions à propos des affaires publiques, de l'autorité, des conflits passés ou présents, c'est le comédien, seul, qui les rend acceptables ou odieuses; on est dans l'ordre du spectacle, non de la politique, parce que ce type de cinéma, privilégiant la performance actorielle et la présence physique de l'acteur, mise sur l'aisance avec laquelle il habite l'écran.

Les corps politiques se manifestent dans un tout autre contexte. Aucun long métrage ne peut tenir avec un protagoniste acéphale; les traits du héros, parfois dissimulés au début d'un film, sortent très vite de l'ombre. En revanche, les êtres collectifs sont nécessairement dépourvus de figure: une communauté humaine n'a pas de faciès, sa physionomie se révèle à travers ses actes et ses comportements. À l'écran, le corps multiple est capable d'impressionner par son ampleur, voire de convaincre par son élan, mais il n'offre pas de prise au spectateur, il ne lui permet pas de tenter une identification, ni même de se faire une place au sein du groupe. J'emprunte à Nicole Brenez, en les infléchissant, deux expressions qui conviennent aux foules cinématographiques. Elle parle de «corps critiques [...], corps sans modèle»." $\mathrm{II}$ cinéma, les masses se démènent parce que la vie le leur impose, non parce qu'elles appliquent une convention. Payées pour une figuration, elles obéissent aux ordres du metteur en scène. Ou bien, filmées dans le cours de leurs activités normales, elles n’ont ni le désir ni le temps de prendre une pause. Le corps collectif dévoile, dans les films, l'artifice auquel se plie le corps de l'acteur, il offre ainsi une prise critique au sens où il rappelle qu'il n'y a pas de jeu, même de jeu de scène, sans observance de règles.

La norme, dans la fiction cinématographique, est la mise en vedette de quelques personnes qui, entre elles, organisent des échanges formalisés et très peu socialisés. Le cinéma pourtant, dans son âge d'or, remplissait les salles d'auditoires anonymes et muets, il participait, avec l'usine,

II. N. Brenez, De la figure en général et du corps en particulier. L'invention figurative au cinéma, Bruxelles, De Boeck, 1998, p.37-38. 
le train, la caserne, les cités dortoirs, à l'encadrement des individus et à leur rassemblement en vastes ensembles dépersonnalisés. Pendant la durée du spectacle le public, discipliné, s'attachait à des ombres dont il admirait la liberté d'allure, la maîtrise et la souplesse corporelle. Épisodiquement, brièvement, d'autres silhouettes traversaient l'écran; la foule ne se montrait pas indéfiniment docile, les masses mouvantes que nous venons de croiser étaient, pour une large part, agitées par l'histoire. Le cinéma porte la trace d'une uniformisation des corps, du prix dont elle a été payée, des échecs qu'elle a subis.

Les corps filmiques sont aujourd'hui sans défaut, nés d'une épure, stockés dans une réserve virtuelle. Des prétextes techniques semblent justifier leur triomphe: ils obéissent mieux, ils coûtent moins cher que ceux des acteurs. Ne sont-ils rien d'autre que des commodités financières? N'offrent-ils pas, avant tout, une image publicitaire, l'anatomie sans défaut que chacun de nous est invité à se construire? Le $\mathrm{Xx}^{\mathrm{e}}$ siècle a été mobilisateur. Le XXI ${ }^{\mathrm{e}}$ sera peut-être uniformisateur et les images mobiles enseigneront à leurs spectateurs comment ils doivent consommer pour devenir semblables aux corps idéals de l'univers numérisé. 



\section{::: \\ Vérité du désir, vulnérabilité des corps: Boys Don't Cry}

Dans son ouvrage Vie précaire. Les pouvoirs du deuil et de la violence après le II septembre $200 \mathrm{I}^{\mathrm{I}}$, Judith Butler s'interroge:

La question qui me préoccupe, à la lumière des récents événements de violence à l'échelle mondiale, est celle-ci : qui importe en tant qu'humain? Quelle vie importe en tant que vie? Et, enfin, quelle vie est digne d'être pleurée? (p.20)

Son but est de réfléchir à la possibilité de réactions politiques - plutôt que violentes - à la perte. Elle s'intéresse en particulier à la façon dont, aux États-Unis, «la guerre à la terreur» s'est servie de l'autocensure des médias, du déni de la vulnérabilité, et d'une hiérarchisation de la douleur:

Quels mécanismes défensifs contre l'appréhension de la perte peut-on voir à l'œuvre dans la manière désinvolte que nous avons d'accepter les morts militaires avec un haussement d'épaules, une indignation moralisatrice ou encore une claire satisfaction vindicative? (p.32)

Elle ajoute :

Dans quelle mesure les peuples arabes, qui sont en majorité de confession islamique, ont-ils été repoussés en dehors de l'«humain» tel qu'il a été naturalisé, dans son moule occidental, par les œuvres humanistes contemporaines? Quels contours culturels de l'humain sont ici à l'œuvre ? (ibid.)

Si son but est de penser la spécificité de ce contexte politique et culturel, ses arguments se rapportent à des problématiques plus vastes touchant

I. J. Butler, Precarious Life. The Powers of Mourning and Violence, Londres - New York, Verso, 2004. Nous traduisons toutes les citations. 
au corps, à la vulnérabilité et à la douleur. Quelle que soit la manière dont il est conceptualisé d'une culture à l'autre, le corps est, pour Butler, le site de notre commune vulnérabilité humaine. Reconnaître celle-ci, ainsi que la manière dont le travail de deuil fait de nous des agents, permettrait de déplacer les contours culturels qui donnent forme à la violence guerrière contemporaine, et ainsi de pouvoir en combattre les visées déréalisantes. Pour Butler:

Le corps implique la mortalité, la vulnérabilité, la possibilité de devenir agent : la peau et la chair nous exposent au regard des autres, mais aussi au toucher, et à la violence, et nos corps nous font également courir le risque de devenir l'agent et l'instrument de telles forces. (p.26)

Autour du corps s'articulent des questions relatives à la nature et à l'impact de la violence, et il est urgent, si l'on souhaite faire face aux effets du recours systématique à l'action militaire et de l'augmentation des crimes contre les minorités, que ces questions soient mises au premier plan. Un refus de l'effet déréalisant de telles actions va de pair avec une prise de conscience de leur coût comme de la vulnérabilité humaine. Pour Butler, la douleur qu'occasionnent de telles pertes pourrait donner naissance à de nouvelles formes de responsabilité politique.

Cette réflexion sur la vulnérabilité et ce qui est digne d'être pleuré sera au cour de notre discussion du premier film de Kimberly Peirce, Boys Don't Cry (I999), et des questions relatives au corps qu'il soulève. Cette œuvre évoque des événements survenus au cour de l'Amérique et fut tourné avant les événements du II septembre 200I, dont Butler évoque les conséquences dans Vie précaire. Dans son second film, Stop Loss (2008), Peirce traite elle-même de la guerre d'Irak et de la désertion d'un soldat américain. Dès Boys Don't Cry, elle interroge l'humain et la déréalisation, la violence et la douleur, ainsi que le vécu d'une relation dissidente par rapport aux normes et aux diktats de l'hétérosexualité obligatoire. De son côté Butler, dans Vie précaire, reconnaît à quel point la régulation sociale des normes identitaires et la reconnaissance de la valeur d'une vie comme digne d'être pleurée sont utiles pour penser l'identité queer et gagnent ainsi à être rapportées aux réflexions philosophiques contemporaines sur l'identité :

Si quelqu'un est perdu, et qu'il n'accède pas au statut de personne, alors quelle est la perte, où survient-elle et quand le travail de deuil peut-il prendre place? Cette dernière question a sans aucun doute été posée dans les études sur l'identité lesbienne, gay et bisexuelle en rapport à la violence dirigée contre les minorités sexuelles; elle a été posée par les personnes 
transsexuelles alors qu'elles se trouvaient ostracisées, victimes de harcèlement et parfois de meurtre; elle a enfin été également posée par les personnes hermaphrodites, dont les années d'apprentissage sont si souvent marquées par des violences imposées à leur corps au nom d'une conception normative de l'humain, d'une conception normative de ce que doit être le corps d'un humain. (p.32-33)

Ses mots semblent également refléter les problématiques sous-tendant le premier film de Peirce sur le harcèlement et le meurtre d'un individu transgenre. Telle est bien la réussite de Boys Don't Cry : créer l'espace - tout mobile et éphémère soit-il - où pourra prendre place un deuil capable de s'opposer aux conceptions normatives de l'humain.

Boys Don't Cry, qui s'inspire d'une histoire vraie, dépeint les dernières semaines de la vie de Brandon Teena, assassiné dans la nuit du 3I décembre 1993 à Falls City dans le Nebraska. Brandon avait été violé sept jours auparavant. Son identification en tant qu'homme et le succès avec lequel il s'était fait passer pour tel à Falls City furent abruptement occultés par la catégorisation, la stigmatisation et la mortification violentes de son corps par deux hommes avec lesquels il s'était d'abord lié d'amitié. Le film a rapidement fait l'objet de discussions détaillées dans les revues de cinéma, et notamment d'un dossier publié par la revue britannique Screen, qui proposait de très riches analyses des questions de genre et de sexualité explorées par le film, tout en attirant l'attention sur d'autres pistes de réflexion encore peu étudiées - notamment le traitement des questions de race. ${ }^{2}$ Ces discussions m'ont paru ne pas suffisamment mettre en évidence le nœud formé des notions de défense et de déréalisation d'une part, et de vulnérabilité du corps d'autre part. J'étudierai ainsi Boys Don't Cry comme un film révélant le fonctionnement de l'imagination dans la construction de l'intégrité corporelle et dans l'évolution des relations de désir avec l'autre. Dans la partie centrale de l'intrigue - l'histoire d'amour entre Brandon et sa petite amie Lana Tisdel - le film attire notre attention sur l'instabilité corporelle et sa régulation par les fictions normatives de l'hétérosexualité obligatoire. Les frontières du corps et ses relations avec l'autre sont construites et médiatisées par l'imagination. Toutefois, à mesure que le film progresse, il s'avère que les fictions protectrices qui alimentent le désir sont réduites à l'impuissance lorsqu'elles se trouvent confrontées à la fragilité du corps. La grande radicalité du film est ainsi d'élaborer une politique de la vulnérabilité, et cette réussite

2. Voir J. Stacey et S. Street, Queer Screen. A Screen Reader, Londres, Routledge, 2007. 
gagne à être lue à la lumière des réflexions de Butler dans Vie précaire. Il est également possible que Boys Don't Cry, si l'on s'aventure au-delà de Butler et sur un terrain plus délicat, ouvre des pistes pouvant nous mener à une pensée de la charge érotique de la vulnérabilité, lorsque le film nous montre Brandon comme adorable, et, en vertu de cette faiblesse même, digne d'être pleuré. Telles sont les questions que le film pose alors qu'il s'approprie la vraie vie de Brandon Teena pour en faire l'objet d'un deuil public, et repense ainsi le genre de la biographie cinématographique ou celui du docudrama en élégie.

La vie et la mort tragique de Brandon Teena ont acquis un statut de légende contemporaine dans la presse américaine; ils ont donné lieu à la réalisation du documentaire The Brandon Teena Story (Susan Muska et Greta Olafsdóttir, 1998), et ont servi de point de ralliement politique pour les activistes transsexuels. Peirce a entendu parler de l'affaire pour la première fois en lisant un article dans le magazine Village Voice en 1994. Elle admet être tombée amoureuse de Brandon et s'être ainsi engagée dans le projet - qui allait durer cinq ans - de filmer son histoire. Lors de ses recherches préparatoires, Peirce a vécu dans le Nebraska, côtoyé les adolescents de la région et interviewé les survivants de l'affaire - Lana Tisdel et JoAnn Brandon, la mère de Brandon - ainsi que la police. Elle a lu des retranscriptions du procès qui a suivi le meurtre et élaboré une chronologie retraçant l'un après l'autre les événements de l'affaire, à partir de la naissance de Brandon. Le travail qu'elle a entrepris pour évaluer les éléments d'une vie, d'un crime et d'un procès a nourri le film qu'elle en a tiré. Si l'on compare Boys Don't Cry avec The Brandon Teena Story, le documentaire de Muska et Olafdottir qui montre les véritables lieux des faits ainsi que de nombreuses photographies de Brandon et des autres protagonistes, des entretiens avec les survivants du crime filmés chez eux comme avec les assassins de Brandon en prison (John Lotter et Marvin Tom Nissen), il est clair que Boys Don't Cry s'est profondément imprégné de l'atmosphère de l'histoire et de l'endroit où elle s'est déroulée. En passant au format du long métrage de fiction, Peirce a également fait preuve d'une attention remarquable aux détails des voix et des inflexions, au style vestimentaire et au contexte en général.

On a dit de Boys Don't Cry qu'il s'agissait d'une histoire vraie: Fox Searchlights, dans l'improbable descriptif proposé lors de la promotion 
du film, parle d'une «histoire vraie sur l'espoir, la peur et le courage d'être soi-même». Cette accroche paraît particulièrement mal choisie lorsqu'on lui oppose les faits de l'histoire de Brandon. ${ }^{3}$

Brandon Teena est né Teena Renae Brandon en décembre 1972. Sa mère JoAnn Brandon avait seize ans et avait déjà une petite fille de trois ans; son père avait trouvé la mort dans un accident de voiture alors que JoAnn n'était enceinte que d'un mois. Élevé comme une petite fille à Lincoln dans le Nebraska, Brandon eut une enfance instable et fut victime d'abus sexuels. Adolescent, il remit en question sa sexualité et son identité en tant que femme. Le genre de l'objet de son désir resta stable: Brandon eut plusieurs petites amies successives, des filles de la région dont les plus jeunes entraient dans l'adolescence et les plus âgées avaient une vingtaine d'années. Beaucoup offrirent des témoignages d'amour à Brandon après sa mort. Brandon refusait cependant de se voir attribuer une identité lesbienne; on a rapporté qu'il aurait déclaré être une fille mais avoir tous les sentiments et toutes les intentions d'un homme. Dès l'âge de dix-huit ans, Brandon se travestit, s'identifiant apparemment à un transsexuel avant opération. Témoignent de la confusion cognitive que suscita son identité les fluctuations constantes entre pronoms masculins et féminins dans les récits de son histoire par les médias, ainsi que la fascination malsaine dont fit l'objet la vérité de son corps sexué. Brandon lui-même emprunta une série de noms et endossa un certain nombre de fausses identités: Billy Brinson, Teena Rae Brandon et Brandon Teena. On a rapporté cette succession d'identités à ses activités de petit délinquant en joignant déviances sociale et sexuelle.

Ces faits, et les détails concrets recueillis par Kimberly Peirce forment la substance de Boys Don't Cry. Le succès du film tient à la manière dont elle a su transformer ce matériau en histoire d'amour et en légende d'une part, et en enquête sur la vulnérabilité et la commémoration d'autre part. Dans la revue Sight and Sound, le journaliste Danny Leigh demande à Peirce: «N'avez-vous pas eu peur que le public ne souhaite que la frontière entre fait et fiction soit plus claire?»*; cela ne semble pas avoir préoccupé Peirce. On peut dire que Boys Don't Cry, dans la mesure où sa réalisatrice a refusé de tracer une frontière claire entre histoire et fiction, témoigne d'une conscience métafictionnelle du statut des films qui

3. A. Jones, All She Wanted. The True Story of Brandon Teena, New York, Pocket Books, 1996.

4. D. Leigh, «Boy wonder», Sight and Sound, vol. IO, n ${ }^{\circ}$, mars 2000, p. I8-20 et particulièrement p. I8. 
touchent à la représentation de l'histoire. Certes, le caractère récent des événements narrés par le film suggère qu'il pourrait être erroné de considérer Boys Don't Cry comme un film historique; cependant, comme le rappelle Vivian Sobchack, «le rapport entre l'événement et sa représentation, l'immédiateté et la médiation, tend de plus en plus vers la simultanéité ». 5 Dans son article «The modernist event», Hayden White attire notre attention sur le fait que la représentation réaliste d'événements historiques est de plus en plus souvent traitée dans les ouvres postmodernes, précisément par l'abandon de l'opposition entre fait et fiction. Il remarque :

C'est cette dimension du modernisme qui informe la création de nouveaux genres, sous forme écrite comme visuelle, de représentation post-moderne, para-historique, que l'on a appelés successivement «docudrama», «fact/ fiction», «infotainment» [littéralement «infodivertissement»], «fiction du fait», «métafiction historique », et ainsi de suite. ${ }^{6}$

Dans cette optique, on peut voir Boys Don't Cry comme un film qui, par le contexte culturel dominant dans lequel il s'inscrit, et conformément aux fantasmes de ses protagonistes, épouse lui-même une forme de regard autocritique sur le caractère inextricable de la relation entre fait et fiction. Le film traverse ainsi les barrières génériques entre fiction et documentaire lorsque, dans sa propre diégèse, il retourne avec insistance à la question de la vérité comme à celle de la fiction.

À cet égard - et parce qu'il a été réalisé avant le II-Septembre -, on peut considérer Boys Don't Cry comme le précurseur d'une tendance qui domine le cinéma indépendant américain pendant les premières années du $\mathrm{XXI}^{\mathrm{e}}$ siècle. On peut le comparer au film Monster de Patty Jenkins (2003), qui s'inspire de l' «histoire vraie» d'Aileen Wuornos, une prostituée de Daytona Beach qui devint tueuse en série. Dans son exploration de l'amour entre Aileen et sa petite amie Shelby, ce film fait écho à la romance queer de Boys Don't Cry. Le rôle d'Aileen permet également à Charlize Theron de se soumettre à une métamorphose corporelle qui représente un défi créatif comparable à celui relevé par Hillary Swank dans son incarnation transgenre de Brandon dans Boys Don't Cry. Dans les termes de White, et au-delà des frontières de genre, Boys Don't Cry présente aussi d'étranges affinités avec des explorations de la vérité et de

5. V. Sobchack, «Introduction. History happens", The Persistence of History, V. Sobchack éd., New York - Londres, Routledge, I996, p. I-I4 et particulièrement p. 5.

6. H. White, «The modernist event", ibid., p. I7-38 et particulièrement p. I8. 
la fiction aussi dérangeantes que le Capturing the Friedmans d'Andrew Jarecki (2003) ou le Last Days de Gus Van Sant (2005).

Peirce s'interroge ainsi dans Sight and Sound: "Comment pourriezvous jamais dire à un public ce qui est ou n'est pas vrai ? " Elle poursuit: «Je dirais que c'est émotionnellement vrai. » «Je voulais entrer en contact avec Brandon et son désir, explique-t-elle. Car le désir est la seule vérité - si quelqu'un entre dans la pièce et que vous le désiriez, cela au moins est vrai. ${ }^{7}$ Dans ces mots comme dans les sensuelles chansons de country de la bande-son et les plans baignés de désir s'arrêtant avec insistance sur les échanges entre Brandon et ses petites amies, Peirce évoque un ancrage de la vérité dans l'expérience corporelle: non pas la vérité du sexe ou du genre, que l'histoire de Brandon conteste, mais la vérité du désir, c'està-dire d'une passion pour un corps vulnérable.

Dans sa présentation de l'histoire de Brandon Teena, Peirce énonce une vérité tout en prétendant mentir: celle du désir et de la vulnérabilité dans une biographie qui s'éloigne de la vérité de la vie de Brandon. À propos du Décalogue 8 de Krzysztof Kieslowski (1989) et du commandement «Tu ne commettras point de faux témoignage contre ton prochain», Slavoj Zizek écrit :

La «vérité» de ce commandement concerne la tension proprement dialectique entre dire la vérité et mentir: on peut mentir en prétendant dire la vérité (c'est ce que font les personnalités obsessionnelles lorsque, dans des énoncés parfaitement exacts sur le plan factuel, ils cachent ou renient leur désir), et on peut dire la vérité en prétendant mentir (la procédure hystérique, ou lorsqu'un simple lapsus trahit le véritable désir du sujet). ${ }^{8}$

C'est dans les passages de Boys Don't Cry qui semblent les plus éloignés de la vérité que les vérités qu'expose le film sont mises en évidence de la façon la plus criante. Zizek poursuit:

Commettre un faux témoignage contre son prochain n'est pas avant tout une question d'exactitude factuelle, mais du désir qui alimente ma position d'énonciation lorsque je dis la vérité (ou lorsque je mens) : ainsi, si je dénonce la femme de mon voisin à son mari en l'accusant d'adultère et par là même (peut-être) je détruis leur vie, cette accusation, même si elle est «vraie» factuellement, est fausse si et dans la mesure où elle est alimentée par mon désir pour elle, par ma «convoitise de la femme de mon prochain». Je le fais par jalousie, parce que ce n'est pas moi qu'elle a pris pour amant. (Ibid.)

7. D. Leigh, «Boy wonder», art. cité, p. I8.

8. S. Zizek, The Fright of Real Tears. Krzysztof Kieslowski between Theory and Post-theory, Londres, BFI Publishing, 200I, p.II7. 
Peirce recourt à la narration de l'histoire de Brandon, et du désir comme vérité, pour alimenter la vérité de son film. Celle-ci réside dans son désir pour Brandon, qui l'incite à pleurer la mort de ce dernier et à proclamer sa vie comme digne d'être pleurée.

Brandon, tel qu'il est imaginé par Peirce, est construit comme une figure qui brouille les frontières entre vérité et fiction: dans sa performance transgenre, dans les crimes auxquels il prend part volontairement ou involontairement, et, plus richement encore, dans la façon dont il réalise une série de fantasmes hétérosexuels romantiques. Le questionnement de Pierce sur la vie affective de Brandon, sur le fantasme intersubjectif qu'il produit et que sa petite amie Lana alimente, permet au film de prendre acte, à un niveau plus complexe, de certaines vérités sur la relation amoureuse. Le film dissipe la véracité et suspend la vérité de manière à pouvoir mener ce questionnement sur les structures du fantasme. Cette exploration n'opacifie en rien l'économie politique du film, mais au contraire participe pleinement de son analyse de l'hétérosexualité obligatoire, des morphologies bi-genres normatives des corps, et de la transphobie ou de l'homophobie. Les passages où le film s'éloigne du réalisme - la représentation onirique de l'autoroute américaine contre le ciel bleu électrique et ses motifs de néons et de reflets de phares entrelacés, le retour élégiaque aux «yeux les plus bleus du Texas» de la chanson - produisent eux aussi un effet de commémoration poétique de Brandon.

Je me souviens de la première fois que j'ai entendu parler de Brandon: c'était dans un article du New Yorker en I995. Je fus fascinée par les questions que soulevait l'article à propos de ses petites amies. Comment ces filles vivaient-elles leur amour pour Brandon? Que connaissaient-elles, que demandaient-elles au sujet de la vérité ou de la fiction de son genre ou de sa sexualité? Que disaient leurs relations amoureuses de leur identification hétérosexuelle ou homosexuelle? Boys Don't Cry maintient rétrospectivement l'image fantasmée de Brandon telle qu'elle a été diffusée par ces anciennes petites amies. Dans le prologue du film, une séquence à l'atmosphère onirique semble tout droit sortie de la plus classique des comédies romantiques. On y voit Brandon se faire passer pour un garçon pour la première fois. Il progresse vers les lumières scintillantes et l'espace circulaire d'une piste de patin à roulettes où il a rendez-vous avec une jeune 
fille blonde nommée Nicole. Nous les voyons patiner ensemble. Nicole dit à Brandon qu'il doit venir d' "un bel endroit» et le plan suivant capture la radieuse série de reflets bleus et scintillants que projette une boule lumineuse de discothèque. Brandon se conforme en tous points au modèle de petit ami que donnent à voir de tels films. Il raccompagne Nicole chez elle; il flirte avec Candace, une mère célibataire de Falls City; il vole une bague à Lana et la laisse posée délicatement en équilibre sur l'oreille d'une vache en peluche sur le lit de la jeune fille. Brandon est de ces romantiques qui brisent les cœurs, et, d'un bout à l'autre de ces premières scènes, Peirce le positionne comme un sujet masculin pris entre les regards successifs des femmes qu'il désire. Une étonnante réussite de Boys Don't Cry, dans ces épisodes où le fantasme de l'identité masculine de Brandon est impeccablement entretenu, est bel et bien de ré-enchanter l'hétérosexualité.

Dans son incarnation du parfait petit ami, on voit Brandon donner corps à la figure fantasmée de l'homme. Le film semble ici rejoindre les réflexions de Zizek sur le fantasme et son rôle dans les relations amoureuses. Dans Enjoy your symptom! Zizek écrit:

Dans le réseau des relations intersubjectives, chacun d'entre nous est identifié à, catégorisé comme, un certain espace fantasmé dans la structure symbolique de l'autre. La psychanalyse maintient ici l'exact opposé de l'opinion ordinaire et banale selon laquelle les figures fantasmées ne sont rien d'autre que des figures déformées, recomposées ou concoctées de quelque autre manière à partir de modèles «réels", d'êtres de chair et de sang que nous avons rencontrés dans notre expérience. Nous ne pouvons entrer en contact avec ces «êtres de chair et de sang» que dans la mesure où nous sommes capables de les identifier à une certaine place dans notre espace symbolique du fantasme, ou, pour le dire de façon plus pathétique, seulement dans la mesure où ils peuvent remplir une place préétablie dans notre rêve - nous tombons amoureux d'une femme parce que ses caractéristiques lui permettent de coïncider avec notre figure fantasmée de la Femme. ${ }^{9}$

Dans les premières parties du film, Peirce nous montre comment Brandon, dans ses poursuites romantiques, tente de coïncider avec la figure masculine fantasmée par sa petite amie. Zizek poursuit dans The Plague of Fantasies:

Tout contact avec un autre «réel», de chair et de sang, tout plaisir sexuel que nous prenons à toucher un autre être humain, n'est pas quelque chose

9. S. Zizek, Enjoy Your Symptom! Jacques Lacan in Hollywood and Out, New York - Londres, Routledge, I992, p.5-6. 
d'évident mais de traumatique par sa nature même, et ne peut être entretenu que dans la mesure où cet autre rentre dans le cadre fantasmatique du sujet. ${ }^{10}$

Boys Don't Cry illustre la manière dont le fantasme entretient la relation amoureuse, soulignant ainsi le traumatisme inhérent à toute relation à l'autre et les façons dont le fantasme travaille à le cacher et le nier.

Le film nous montre comment le fantasme de masculinité idéale de Brandon (qui se montre chevaleresque, beau parleur, attentif) alimente sa relation avec Lana. Ce fantasme de l'être masculin parfait est, de façon plus troublante, précisément ce qui menace les autres hommes dans le film, les amis, rivaux et assassins de Brandon, John Lotter et Thomas Nissen, qui sont bien loin de s'approcher de l'idéal romantique et érotique de Lana. À travers les tentatives de Lotter et de Nissen pour détruire le fantasme qu'a conçu Brandon de sa propre masculinité, le film va plus loin que Zizek pour imaginer comment, dans des contextes sociaux et politiques différents, certaines relations amoureuses sont plus susceptibles de perdurer que d'autres. Le fantasme de masculinité de Brandon nie la disjonction entre son genre (masculin) et son corps sexué (féminin). Le film entreprend d'interroger les manières par lesquelles la réalisation de tels fantasmes est rendue impossible ou prohibée au sein du modèle de l'hétérosexualité obligatoire. Dans ce contexte politique et social, la relation amoureuse est ironiquement menaçante parce qu'elle s'approche d'un idéal de romance hétérosexuelle et qu'elle remet ainsi en question les notions figées de vérité du sexe et du genre. Ces questions sont soumises à un riche examen critique dans une scène que je souhaite examiner de plus près.

La scène intervient dans le dernier tiers du film et se passe la nuit du viol de Brandon par John Lotter et Thomas Nissen. Lana rentre chez elle pour trouver sa mère et les membres de sa famille confrontés à la preuve que Brandon est une femme : le journal évoque sa comparution devant la cour de justice sous le nom de «Teena Brandon». Cette preuve juridique va à l'encontre de leur croyance familière que Brandon est un homme. La scène est marquée par une forte transition en termes de style visuel. Elle s'ouvre dans les espaces blancs de la maison familiale; la caméra à la main suit la dispute et la colère qui se propagent parmi les personnages. On a comparé le style visuel de Peirce à celui de John Cassavetes dans

IO. S. Zizek, The Plague of Fantasies, Londres, Verso, 1997, p. 65. 
Une femme sous influence ( $A$ Woman Under the Influence, 1974). Rejoignant ce type de réalisme exigeant, cette partie du film montre les personnages en train de tenter d'établir la distinction entre vérité et fiction. John Lotter demande à Brandon à plusieurs reprises: «Est-ce que t'es une fille, oui ou non? » Lana interrompt cet interrogatoire et s'interpose entre John et Brandon.

Le changement de rythme et d'esthétique s'opère à l'instigation de Lana, lorsqu'elle et Brandon se réfugient dans l'intimité de sa chambre. Dans cet espace, Lana va tenter de garantir la pérennité du fantasme qui alimente sa relation à Brandon. Alors qu'il s'apprête à déboutonner son pantalon pour lui révéler la vérité de son corps, elle lui dit: "Non, non, non. Rhabille-toi. Ne me montre rien. Réfléchis. Je sais que tu es un mec. » C'est la réalité que Lana peut construire avec Brandon. Elle s'assied sur son lit, le regarde de ses grands yeux bleus; sa tête se découpe contre la lumière. Alors qu'il commence à lui expliquer son identité par les mots: "Je suis né avec ce truc bizarre...», elle attaque son propre récit de la vérité en disant: «Moi aussi je fais des tas de trucs vraiment bizarres. » La douceur de ses mots et son calme œuvrent à nier la violence de la scène précédente. Elle dit à Brandon de ne pas avoir peur et choisit plutôt de l'attirer dans l'espace fantasmatique de l'autre côté de la fenêtre en lui disant: «Regarde comme c'est beau dehors.»

Le film passe à l'un de ces plans caractéristiques, où se juxtaposent, en une série d'ellipses temporelles, des images de l'autoroute dans la lumière bleu pâle de l'aube naissante. La répétition du motif du «bel endroit» et le retour du bleu scintillant nous rappellent la scène de patin à roulettes au début du film, la première incursion du film dans le fantasme de masculinité de Brandon. La caméra se déplace vers le haut pour s'arrêter sur les nuages qui passent à l'horizon. Avec pour toile de fond cette image intersubjective du rêve - l'espace filmique du paysage américain -, Lana vient au secours du défaillant fantasme viril de Brandon, de son incarnation de sujet mâle mise en danger. Le style visuel du film se fait différent lors de ces moments, où Lana parvient à établir un contact avec Brandon à travers leur commun fantasme de relation hétérosexuelle. Le film établit une coupure littérale avec l'espace domestique de la maison de Lana, espace auquel elle-même comme Brandon ont tenté d'échapper. Elle déclare: "Je vais leur dire ce qu'on sait tous les deux être vrai.» Leurs deux visages sont filmés en très gros plan lorsqu'elle fait cette promesse. Brandon se tourne vers Lana, qui sourit; la mise au point se fait sur celle-ci tandis que l'image de Brandon se brouille légèrement. Le film 
se recentre sur Lana qui témoigne de la vérité du désir, celle de son fantasme: "Maman. Je l'ai vu en chair et en os. " J'l'ai vu. Je sais que c'est un homme.» Mais alors que le film s'éloigne de l'espace symbiotique de la chambre de Lana et de son fantasme préservé avec Brandon, le charme qu'elle a jeté est rompu. Le dialogue qui suit nous ramène à la quête de la vérité. La mère de Lana rétorque: "Je veux la vérité. Qu'est-ce que t'es, bon Dieu?» John Lotter, qui tient Brandon à la gorge, lui enjoint: «C'est vrai, mec. Allez, dis-nous.»

Cette scène, ainsi que les transitions qu'on peut y observer, pourrait à mon sens signaler la complexité du film dans son retour aux questions relatives à la vérité. Le fantasme de Lana, une fois qu'elle se heurte à la quête de vérité de sa famille, ne peut plus la protéger ou protéger Brandon du traumatisme de leur contact avec le monde extérieur. Le film nous montre même que les dénis de Lana et son entretien du fantasme sont potentiellement dangereux. Sa relation à John Lotter, par exemple, témoigne de son déni de cette menace: «John, dit-elle, personne va tuer personne, OK? » À propos de la véritable Lana Tisdel, Peirce remarque dans Sight and Sound: "Elle a fait l'erreur de jugement de ne pas se dissocier de John et Tom, et Brandon se retrouve mort. $\rangle^{\text {II }}$ Toutefois, bien qu'elle reconnaisse le caractère dangereux de son déni, je pense qu'en dernière analyse le film se range aux côtés de Lana.

Dans sa façon de remanier et d'embellir esthétiquement une «histoire vraie ", Peirce elle-même, comme Lana, choisit de souscrire à une vision fantasmée qui occulte certaines vérités de manière à maintenir et alimenter une vérité du désir. C'est l'acte d'amour du film, et il s'avère que c'est également un acte de commémoration. Le film donne à voir les dénis de Lana, et pourtant, par son insistance sur Brandon, n'échappe pas lui-même à ses propres dénis. De cette manière, le film nous montre tout à la fois comment nous nous aimons les uns les autres et déplore la manière dont un tel amour est toujours mortellement dangereux quand il remet en question l'hétérosexualité obligatoire. Pour Peirce, l'histoire vraie fera toujours fusionner histoire et fiction. À travers leur interrelation, Boys Don't Cry peut encore témoigner d'une réalité sociale et politique de l'homophobie et, de façon plus émouvante encore, d'une vérité du désir. Il reste à voir quelle place occupe ici la vulnérabilité dans un amour si tragique.

I I. D. Leigh, «Boy wonder», art. cité, p. 20. 
J'ai montré la manière dont le film s'éloigne de la réalité dans sa mise en scène de l'amour de Brandon et Lana. Il parvient à mettre leurs fantasmes en relation avec une fascination plus large pour les atteintes au corps et la vulnérabilité. Boys Don't Cry s'intéresse aux défenses contre l'angoisse de la perte, mais il se préoccupe aussi, viscéralement, du dommage physique qu'occasionnent ces pertes. Dans sa manière de filmer le viol et le meurtre de Brandon Teena, Peirce met clairement en évidence leur sauvagerie et leur horreur. Si j'ai mentionné Cassavetes, et que Peirce elle-même parle de l'influence de Gus Van Sant sur ses choix de réalisatrice, la fascination dont témoigne son film pour le corps dans des états extrêmes et vulnérables peut appeler une comparaison avec d'autres réalisateurs mentionnés dans cet ouvrage, par exemple Bruno Dumont et les frères Dardenne. Peirce partage leur intérêt pour le corps comme canal et comme écran.

Les problématiques touchant à la représentation du corps qui ont jusqu'ici largement dominé l'analyse de Boys Don't Cry ont été articulées à des questions de sexualité, d'identité transgenre et de morphologie corporelle. De tels niveaux d'analyse dérivent de l'impossibilité de répondre à l'agressive question de John Lotter: «Est-ce que t'es une fille, oui ou non? » La quête réductrice et essentialiste qu'il entreprend avec Tom Nissen, pour découvrir, par l'examen et la violation de son corps, la vérité de l'identité de Brandon, offre un contraste saisissant avec la reconnaissance (queer) par le film que l'identité de genre et la morphologie corporelle reflètent une superposition d'identifications et résultent d'une construction performative. Comme l'a souligné Butler dans son travail depuis Trouble dans le genre ${ }^{\mathrm{I2}}$, les identifications transgenres dérangent les modèles sociaux qui constituent le fondement de l'hétérosexualité obligatoire. Brandon, le personnage de Peirce, qui fait l'expérience, pour reprendre ses propres mots, d'une «crise d'identité sexuelle» en s'identifiant à un homme malgré sa morphologie corporelle apparemment féminine, cherche à maintenir une incarnation intelligible de masculinité qui ne soit pas une mascarade, mais l'expression de son identité véritable. Comme il le dit, avec Lana, «enfin tout semblait être dans l'ordre des choses». Dans les mots de Michelle Aaron, «la véritable identité de Brandon,

I2. J. Butler, Gender Trouble. Feminism and the Subversion of Identity, New York - Londres, Routledge, 1990. 
c'est-à-dire son identité transgenre, n'est jamais dérobée au regard du spectateur ». ${ }^{13}$ C'est la vérité de l'identité de Brandon telle que le film la maintient. Et les spectateurs sont invités à constater le travail nécessaire au succès de cette métamorphose identitaire tandis que Brandon est confronté à l'incongruité de ses seins, qu'il bande, et de son sang menstruel. C'est bel et bien une contestation de la construction normative de l'identité corporelle que le film élabore, si l'on se place à un certain niveau de lecture, politique. Reste cependant à voir de quelle manière la fascination plus profonde du film pour les dommages au corps met en perspective cette prise de position.

Dans ses réflexions sur la question des vies dignes d'être pleurées, et sur la violence physique, Butler fait spécifiquement référence à la communauté gay, lesbienne et transgenre. Il semble également être reconnu que de telles vies sont peut-être plus vulnérables et plus susceptibles d'être en butte à la violence des crimes contre les minorités, comme le viol et le meurtre de Brandon Teena semblent en effet clairement le souligner. Le film identifie l'homophobie de ses personnages lorsque Tom dit à John, la nuit du meurtre, qu'ils ne vont faire que «s'occuper de deux gouines». Il fait ici référence à Brandon et au personnage de Candace, transposition de Lisa Lambert, qui fut également tuée à bout portant. Le film revendique certainement le statut des vies queer comme dignes d'être pleurées, et par là même conteste l'homophobie et la transphobie.

Judith Halberstam a cependant fait remarquer que le film Boys Don't Cry représente de façon fallacieuse le crime de John Lotter et de Tom Nissen puisqu'il choisit de taire le meurtre d'une troisième personne qui se trouvait ce soir-là dans la maison de Lisa Lambert. Halberstam évoque la manière qu'a le film de "passer sous silence l'histoire de l'autre victime qui mourut aux côtés de Brandon Teena et de Lisa Lambert. Il s'agissait de Philip De Vine, un handicapé d'origine afro-américaine ( ibid., p. 28I). Comme d'autres, elle critique Peirce pour avoir omis de mentionner cet homicide. Une certaine hiérarchie de perspectives semble voir le jour là où Boys Don't Cry, qui met en cause l'homophobie et la transphobie, met au premier plan l'amour de Lana et Brandon et fait ainsi de ce dernier un martyr transgenre tout en occultant d'autres crimes haineux commis dans la maison de Lisa Lambert. Halberstam évoque les liens de l'un des tueurs à une organisation prônant la suprématie de la race blanche. Les argu-

I3. M. Aaron, «Pass/fail», Queer Screen, J. Stacey et S. Street, ouvr. cité, 2007, p. 259-264 et particulièrement p. 260 . 
ments convaincants qu'elle fait valoir pour dénoncer le racisme - volontaire ou involontaire - du film, ainsi que ceux présentés par Brody (p. 289295), révèlent les dangers de toute hiérarchisation de la victimisation. Il est possible que Peirce ait tort d'ôter Philip De Vine du récit dans Boys Don't Cry. Son erreur tient peut-être à une tentative de dissocier la discrimination et la haine envers les groupes minoritaires selon le genre ou la sexualité de celle dirigée contre les autres minorités. Par-delà l'histoire «vraie» de Brandon Teena et sa représentation «falsifiée» à l'écran, la vérité des motivations, les complexités de la haine et du désir, le désordre du réel vont au-delà des dynamiques binaires du cinéma narratif, quelles que soient sa richesse et sa force émotionnelle. Montrer le meurtre de Philip De Vine permettrait de suggérer que la vie de Brandon est vulnérable et digne d'être pleurée non seulement du fait de son identification transgenre politiquement chargée, mais aussi parce que, comme d'autres individus «différents», il figure un «dehors» aux catégories identitaires normatives. Ce serait faire de lui la victime d'un crime haineux à motivation large nous amenant ainsi à examiner les catégories de l'humain en général.

En dépit de ces omissions, je pense que le film s'efforce d'attirer l'attention sur de telles catégories, et en particulier, à travers son souci de représenter la vulnérabilité de Brandon, de montrer son corps comme vulnérable face aux atteintes physiques. C'est bien là que réside la complexité de ce thème dans le film: celui-ci s'expose à certains risques en confirmant le statut de victime d'un héros transgenre, mais demeure néanmoins éthiquement convaincant dans sa manière d'utiliser la vulnérabilité comme un moyen de contester la violence. Dès le début du film, une série de blessures physiques marque les mouvements de Brandon pendant les dernières semaines de sa vie. La nuit où il rencontre Candace, il reçoit des coups lors d'une altercation dans un bar, et la caméra s'attarde sur les bleus de son visage. Avec Lana, Candace, John Lotter et Tom Nissen, Brandon va s'adonner au sport local, qui consiste à s'attacher à l'arrière d'une voiture en un avatar de ski nautique, sur les routes poussiéreuses du Nebraska. Si le film semble fasciné par la vélocité avec laquelle il «skie» derrière la voiture, il capture aussi avec minutie ses chutes, lorsqu'il glisse au sol, se lève, tombe à nouveau, et ainsi de suite. Les contours de son corps et sa relation au monde sont à plusieurs reprises déterminés par un impact brutal. Lana lui demande pourquoi il laisse John l'attacher à l'arrière du camion et le traîner comme on traînerait un chien. Le courage et les bravades de Brandon semblent à Lana proches d'une violence automutilatoire. De tels éléments peuvent perturber une 
perspective transgenre, puisque le spectateur peut en conclure que ce corps molesté est imparfaitement mâle. Mais, comme le spectateur s'en aperçoit rapidement, le film récuse efficacement toute association figée entre la vulnérabilité et le féminin en tant que tel. Ce n'est pas parce que Brandon n'est pas un homme, un «vrai», qu'il est montré comme vulnérable. Ironiquement, sa vulnérabilité même, sa peau meurtrie et marquée de cicatrices établissent un rapprochement avec un autre homme, Tom Nissen, dont les expériences d'automutilation sont mises en avant dans le récit. Tom dit en effet que se couper lui permet de «revenir à la réalité». La violence que déplore le film - le viol et le meurtre de Brandon - est très certainement mise en œuvre au nom d'une hégémonie blanche et mâle, mais elle est vue comme le produit de l'impuissance littérale et figurée de John et de Tom, comme faisant partie de leurs propres tentatives pour renflouer une virilité rudement malmenée par le désir de Lana pour Brandon. John et Tom, qui parce qu'ils ont fait de la prison peuvent être vus comme abîmés et dangereux, sont eux-mêmes stigmatisés; le danger qu'ils présentent provient bien de leurs tentatives de désavouer les blessures dont ils ont souffert. On les voit, comme en écho aux mots de Butler cités au début de cette contribution, se transformer en instruments de violence dès lors qu'ils nient leur propre vulnérabilité. ${ }^{14}$

Boys Don't Cry s'intéresse plus spécifiquement à la vulnérabilité de Brandon, ce qui est particulièrement tangible dans les scènes postérieures au viol, et dans les attentions féminines dont Brandon fait l'objet. Dans l'une d'entre elles, une infirmière coupe le pansement qui lui bandait la poitrine avec tendresse, tentant de l'apaiser, sa main gantée de latex posée sur la joue meurtrie. Une autre scène distille plus encore cette fascination pour la vulnérabilité : c'est celle où Lana et Brandon font l'amour dans une grange, peu de temps après le viol. La scène a le rythme lent et l'aura envoûtante d'un rêve ou de la réalisation d'un vœu. Lana est d'une douceur extrême avec Brandon; elle le tient dans ses bras, pose sa tête contre ses seins. Ses doigts délicats lui caressent les cheveux alors qu'ils évoquent l'enfance de Brandon. Cette séquence évocatrice d'une figure de pietà ainsi que la scène de sexe qui la suit - mais n'est pas montrée à l'écran - ont malgré leur aura d'irréalité, suscité des objections.

I4. The Brandon Teena Story attire l'attention d'une façon différente sur la vulnérabilité de John Lotter, en donnant les émouvants témoignages d'un travailleur social et d'un parent adoptif. Le documentaire s'efforce de montrer chacun de ses sujets comme partiellement innocent et vulnérable grâce à des photographies en gros plan qui les représentent enfants. 
La véritable Lana Tisdell, qui a poursuivi sans succès les producteurs du film, soutient que son personnage n'a pas fait l'amour à Brandon après avoir appris qu'il était une femme. La critique Judith Halberstam trouve la scène problématique pour d'autres raisons: elle se demande pourquoi Brandon voudrait faire l'amour si peu de temps après son viol. Plus généralement, cette objection porte sur la perte de l'identité transgenre de Brandon dans cette scène: «Soudain, et de manière catastrophique, Peirce prive son personnage de son regard transgenre et convertit celui-ci en un regard lesbien, et donc en un regard féminin. $\gg^{15} \mathrm{Il}$ n'est peut-être pas tout à fait clair que Brandon s'identifie alors à une femme, ou que son rapport sexuel avec Lana soit identifié comme lesbien. La tendresse dont elle fait preuve envers lui semble plutôt exacerbée par la fragilité nouvelle de son corps et par le terrible pathos qui entoure sa vulnérabilité physique. Lana, qui a reçu plus qu'elle n'a donné du plaisir dans les rapports sexuels qui ont précédé, va à présent faire l'amour à Brandon. Elle lui dit: "Je ne sais pas si je vais savoir comment faire», apparemment en référence à son manque d'expérience avec les femmes, ou peut-être tout simplement à son manque d'expérience à donner du plaisir à Brandon. Cet autre, aimé et blessé, est désormais difficile à toucher, et on voit le désir qu'éprouve Lana. Le pathos de sa vulnérabilité est une composante étrange de leur amour puisqu'il participe de l'attention que le film porte à son sujet.

Il est important de ne pas oublier que Boys Don't Cry est un film sur les morts. C'est un film qui honore la mémoire du véritable Brandon Teena et qui explore la troublante réanimation cinématographique de son corps. Julianne Pidduck observe avec pertinence que «la puissance émotionnelle et l'abord problématique de Boys tiennent à la tension entre la mythologie dynamique du road movie et la réalité persistante de la mort de Brandon Teena» (ibid., p. 265). La réalité de cette mort et les souffrances véritables de Brandon Teena sous-tendent nécessairement le romantisme du film et sa fascination pour la vulnérabilité. La mort tragique et prématurée de Brandon, et le pathos dont elle colore son histoire sont eux-mêmes à l'œuvre dans la représentation de Brandon comme sujet désiré et désirant.

I5. J. Halberstam, "The transgender gaze in Boy's Don't Cry», Queer Screen, J. Stacey et S. Street, ouvr. cité, p. 278-282 et particulièrement p. 280. 
Dans son essai «Loving a disappearing image », Laura Marks ${ }^{16}$ interroge la relation entre la mort et l'art cinématographique. Elle établit un rapprochement entre les images ayant vocation à disparaitre - le Celluloïd et les images de cassette vidéo qui périssent littéralement avec le temps et les images présentes et rétrospectives de la mort et des morts. Elle nous rappelle que «l'histoire du cinéma [...] est dès l'origine un acte mélancolique; car, même en présence de l'image dans sa plénitude, nous sommes conscients qu'elle disparait sous nos yeux» (p. 93). La reconnaissance de la fragilité du médium et de son support va de pair avec la conscience de la pertinence, du caractère adéquat du film comme instrument de deuil et de commémoration. Selon elle, «les films et vidéos indépendants, expérimentaux ou rares ont déjà une aura propre, comme le savent tous ceux qui ont jamais tenté de remplacer des films familiaux perdus » (p.94). Boys Don't Cry, dont l'esthétique ménage une place importante au détail quotidien fourni par les photographies encore existantes de Brandon Teena et par des séquences montrant ses amis et sa famille, tente de faire sienne une telle aura. La reconstitution soignée du cadre des événements réels et l'attention minutieuse aux moindres détails - inflexions d'une voix, style physique, décoration personnelle - témoignent de cette tentative de rendre hommage à Brandon, de le traiter comme précieux, et de trouver des moyens tout à la fois délicats et éphémères de commémorer son décès.

L'un des objectifs de Marks dans «Loving a disappearing image » est d'élaborer une théorie «d'un regard d'amour et de perte» (p.9I). Elle s'oppose à la pathologisation de la mélancolie et se tourne vers des conceptions nouvelles - amoureuses - du deuil perpétuel. «Je suis convaincue, affirme-t-elle, que la mélancolie que suscite une image mourante peut générer non de la crainte mais une attention aimante» (p. IO6). Elle s'interroge : «Y a-t-il une façon d'imaginer un acte de deuil perpétuel qui soit en même temps un acte d'amour?» (p. IO7). Et finit par déclarer: «Entrer en contact avec une image qui disparaît invite à une forme de compassion et d'amour infini qui peut également être une manière d'entrer en contact avec les gens et avec la mort» (p. IO9). Ces mots sont à rapprocher des réflexions de Butler sur la vulnérabilité, la douleur et la responsabilité politique. Ces deux théoriciennes font appel à une conceptualisation du corps comme essentiellement vulnérable, meurtri, mortel, comme source de compassion et de responsabilité politique, soit les réactions mêmes que

I6. L. Marks, Touch. Sensuous Theory and Multisensory Media, Minneapolis, University of Minnesota Press, 2002, p. 9I-IIO. 
Peirce tente de provoquer par son évocation mélancolique, romantique même, de la vie et de la mort de Brandon Teena.

Jacques Derrida affirme à propos du toucher et de la photographie, et en référence à La Chambre claire:

Quand Barthes donne une telle portée au toucher dans l'expérience photographique, c'est dans la mesure où ce dont on est privé, justement, aussi bien dans la spectralité que dans le regard porté vers les images, le cinéma, la télévision, c'est bien la sensibilité tactile. Le désir de toucher, l'effet ou l'affect tactile, se voit alors appelé avec violence par la frustration même, appelé à revenir, comme un revenant, dans les lieux hantés par son absence. ${ }^{17}$

La photographie, les photographies, le film convoquent tous un regard d'amour et de perte, un puissant désir de toucher stimulé par la privation inhérente au médium. Le toucher est un sens que le cinéma ne peut aborder que prosthétiquement ou par procuration; le désir de toucher est toujours frustré. D'où ce privilège ironique du cinéma, qui peut montrer cet avatar de l'intouchable: le corps sensible et animé de l'autre une fois mort. Le cinéma nous montre des images qui ne peuvent être touchées, et qui sont émouvantes, touchantes, pour cette raison même. Lorsqu'elles font renaître la vie qui n'est plus de l'autre aimé et perdu, les images cinématographiques sont doublement spectrales.

En parlant des morts, Derrida nous donne à voir le manque de réciprocité dans notre relation à ceux que nous avons perdus. Il écrit: «Et je ne peux pas m'acquitter, je ne peux ni rendre ni échanger à cause de cette absence de l'autre que je ne saurais regarder dans les yeux » (ibid., p. I37). Il est impossible de toucher l'autre, de l'atteindre; en dehors de l'échange et des interactions humaines, l'autre qui est mort nous ouvre à l'altérité, à une infinité qui elle-même reste hors de notre portée: "L'autre qui est mort, a été quelqu'un pour qui un monde, c'est-à-dire une infinité possible ou une indéfinité possible d'expériences, étaient ouvertes. C'est une ouverture » (p. I38). Ce qui conduit Derrida à poursuivre ainsi sa réflexion : "Le mot de "réel" dans ce contexte-là, signifie l'irréductible singularité de l'autre en tant qu'elle ouvre un monde» (p. I39). Les développements de Derrida peuvent être rapportés à ceux d'Elaine Scarry qui évoque, à propos du corps en proie à la douleur physique, «la fracture absolue entre l'appréhension de sa propre réalité et la réalité des autres personnes»:

I7. J. Derrida, B. Stiegler, Échographies. De la télévision, Paris, Galilée-INA, I996, p. I29. 
[...] quand on entend parler de la douleur physique d'une autre personne, les événements qui prennent place à l'intérieur du corps de cette personne peuvent sembler avoir le même caractère lointain que quelque fait profondément souterrain, appartenant à une géographie invisible qui, toute inquiétante qu'elle puisse paraitre, n’a aucune réalité.$^{18}$

Avec Boys Don't Cry, Peirce compose une élégie pour l'autre qui est mort, tout en donnant une représentation de la vie d'un individu qu'infiniment peu de ses spectateurs auront jamais connu ou touché en réalité. Le film est une histoire vraie, même lorsqu'il s'éloigne à certains moments des faits de la vie qu'il représente. Son approche du réel tient finalement à sa reconnaissance de son sujet comme impossible à atteindre ou à toucher, à sa reconnaissance de la réalité de l'autre comme ce qui échappe à notre portée. En montrant les souffrances de Brandon, il évoque la réalité de sa vie et de sa mort comme, en dernière analyse, définitivement hors de notre portée. Peirce fait un recours fréquent aux capacités palliatives du cinéma pour montrer l'histoire d'une vie telle qu'elle est portée par le fantasme, ici le fantasme de la parfaite incarnation du sujet mâle et de la perpétuation d'une mascarade d'hétérosexualité que Brandon et Lana tentent ensemble. Le corps est produit performativement, connu et nourri par l'invocation d'une série d'actes normatifs. Et pourtant, c'est dans son attention aux dommages au corps résultant de performances défaillantes que le film forme son hommage le plus touchant à Brandon Teena. En nous rappelant tout ce que nous ne pouvons pas toucher, tout ce que nous ne pouvons pas connaître de sa douleur et de sa mortification, le film fait de la vulnérabilité du corps de l'autre, d'un corps apparemment hors des catégories de l'humain, son instrument pour faire naître la compassion et un amour tardif, peiné et rétrospectif pour son sujet. C'est un tel deuil perpétuel, nourri par un regard d'amour et de perte, qui permet au film de toucher au plus près une vérité du désir et, peut-être, d'espérer intervenir en faveur du changement politique et social.

I8. E. Scarry, The Body in Pain, Oxford - New York, Oxford University Press, 1985, p.3-4. 


\section{::: \\ Le corps-récit: Julian Hernandez et Carlos Reygadas}

Dans cette contribution nous étudierons les usages du corps chez deux cinéastes mexicains contemporains, Carlos Reygadas (Japón, 2002; Bataille dans le ciel, 2005) et Julian Hernandez (A Thousand Clouds of Peace, 2003; Broken Sky, 2006 ${ }^{1}$. Chez ces deux auteurs, fût-ce de façon bien différente, le corps filmé apparaît avant tout comme puissante métaphore, reléguant au second plan l'histoire du film. Déplaçant l'intrigue, le corps filmé en occupe la place et comme tout l'espace, s'imposant ainsi comme le trajet essentiel à l'avènement des significations de l'œuvre.

En tant qu'appareillage et modalité essentiels à la représentation depuis la fin du XIX ${ }^{\mathrm{c}}$ siècle, le cinéma est constitutif du regard moderne, non seulement comme mode d'enregistrement (caméra) mais aussi comme ail du public, c'est-à-dire comme source et expression de son psychisme. La subjectivité et l'imaginaire sont façonnés par le cinéma, chez ceux qui ont l'habitude de cette expérience. Nous définissons ainsi le cinéma comme appareil sémiotique et espace représentatif, à la fois formé par et formant les cultures. ${ }^{2}$

Au cinéma, le corps peut aisément être pris dans un jeu d'images plus ou moins choquantes et ainsi perturber l'ordre symbolique via le fantasme et l'imaginaire. Dans un film, un corps n'est pas qu'un corps; il est aussi marqueur de significations symboliques et sociales. L'organisation libidinale du corps dépend de, et en retour est produit par, nombre

I. Mil Nubes de paz cercan el cielo, amor, jamás acabarás de ser amor (A Thousand Clouds of Peace); Cielo Dividido (Broken Sky).

2. Voir B. Nichols, Ideology and the Image, Bloomington, Indiana University Press, I98I; T. de Lauretis, Alicia ya no, Madrid, Catedra, 1992. 
d'instances de médiation. Il est site concret de la signification et de ses processus. Cependant le corps cinématographique décuple la puissance symbolique du cinéma du fait de la construction de l'image par le regard cinématographique : emplacement de la caméra, relation au paysage, proportions, etc. Nos perceptions des corps sont toujours médiées par des réseaux de gestes et d'intentions qui les rendent visibles et signifiants. En ce sens, le corps humain est toujours non naturel. Slavoj Zizek ${ }^{3}$ suggère que la psychanalyse offre la première véritable méthode de compréhension de cette corporalité non naturelle, celle d'un corps doté d'une opaque et effrayante intériorité. Si nous pensons que le corps au cinéma reflète toujours plus que lui-même en ce qu'il est le site de significations plurielles et le médium - ainsi que le producteur - de représentations socioculturelles, alors il nous incombe d'en révéler le sens foncièrement multiple. Qui plus est, les représentations d'appartenance ethnique, de genre et de classes sociales sont "parlées" par le corps cinématographique, et ce, de diverses façons, subtiles ou non. Cela ne s'opère pas seulement, ni même principalement, à travers la diégèse du film, mais aussi par la singularité du langage cinématographique grâce auquel ces corps parlants peuvent être construits et déployés.

Selon les études cinématographiques féministes, depuis Laura Mulvey et après Christian $\mathrm{Metz}^{4}$, le corps (féminin) est le locus du désir (masculin). De plus, le désir est le produit non seulement des impulsions sexuelles mais aussi, et surtout, du regard qui rend ces films prégnants. Au cinéma, l'on peut sentir et lire la complexité du désir, ses anxiétés et ses symptômes, son caractère fantasmatique. La subjectivité moderne et l'imaginaire contemporain sont tous deux formés et déterminés par le cinéma et les images médias au sens large. Le présent essai étudiera comment certains films produisent un corps esthétique (c'est-à-dire correspondant à des canons ou à des règles plastiques plus ou moins explicites), mais aussi comment les normes esthétiques se trouvent elles-mêmes redéterminées par des questions de race et de genres. La blancheur, comme produit de la domination de Hollywood dans la culture populaire mondiale, fait partie de ce que l'on peut appeler, avec Annette Kuhn, le «modèle

3. S. Zizek, Enjoy your symptom! Jacques Lacan in Hollywood and Out, New York, Routledge, 1992.

4. L. Mulvey, "Visual pleasure and narrative cinema", Issues in Feminist Film Criticism, P. Erens éd., Bloomington, Indiana University Press, 1975; C. Metz, El significante imaginario. Psicoanálisis y cine, Barcelone, Gustavo Gilli, I979. 
institutionnel de représentation ${ }^{5}{ }^{5}$ Dans les pages qui suivent nous étudierons la construction de l'image du corps au cinéma comme mode de communication de signifiés, donc rompant le flux de la narration, la rendant secondaire, voire insignifiante. Nous analyserons également comment certains films mexicains parviennent à résister aux lois de la représentation corporelle.

Notre choix s'est porté sur Hernandez et Reygadas parce que leurs films ont été considérés comme subversifs par la critique et qu'ils ont été produits hors des circuits traditionnels, apparaissant en cela comme de vrais films d'auteur en marge de l'industrie du cinéma commercial. Ces deux ouvres portent un regard critique sur la société mexicaine et sur l'ordre social en général, utilisant à cette fin le thème de la sexualité.

\section{Politique de la nation androgyne}

Broken Sky, de Hernandez, est construit autour d'une histoire simple. L'ambition de l'auteur est de faire sentir au spectateur ce que ressent le corps homosexuel lorsqu'il est amoureux. Le corps pervers (homosexuel) est représenté dans ce film comme ayant le droit d'exister. Apparemment simple, sans déterminants moraux ou sociaux, le drame conte la quête de complétude d'un désir androgyne. Hernandez crée un monde utopique où le bizarre est néanmoins bien présent, et menace même la possibilité paradisiaque d'un autre ordre, l'ordre de l'autre.

Le film se déroule à l'université de Mexico, l'UNAM. Deux jeunes hommes, Gerardo (Miguel Angel Hoppe) et Jonas (Fernando Arroyo), deviennent amants et commencent une relation très passionnée. Mais Jonas ne peut s'empêcher de penser à un autre garçon; ce qui plonge Gerardo en dépression jusqu'à ce qu'il soit amoureusement secouru par un employé de l'UNAM (Alejandro Rojo), qui l'admirait en réalité depuis longtemps. C'est alors au tour de Jonas de regretter Gerardo. Ce dernier revient vers Jonas, tous deux sachant qu'ils étaient faits l'un pour l'autre, l'histoire se terminant ainsi «bien» selon l'expression consacrée. Le réalisateur exprime les passions contraires animant Gerardo par de magnifiques images, et le mène - avec le spectateur - vers une vision extatique de l'amour (gay).

5. A. Kuhn, The Power of the Image. Essays on Representation and Sexuality, Londres, Routledge - Kegan Paul, 1985. 
Le précédent film de Hernandez, qui était aussi son premier, $A$ Thousand Clouds of Peace, racontait la même histoire mais dans le contexte du prolétariat mexicain, précarisé et homophobe. Le second film, Broken Sky, devait être placé dans une «île» hors contexte - le campus de l'université de Mexico - afin de se développer en un espace préservé du danger et «esthétique». Les protagonistes, de jeunes étudiants, viennent de la moyenne bourgeoisie et sont libérés du stress de la vie quotidienne. Le film traite de l'obsession de l'amour perdu comme d'un leitmotiv. Dans A Thousand Clouds of Peace, la tragédie amoureuse demeure irrésolue, et l'émotion qui prévaut est la souffrance. Dans le second, nous assistons à un happy end comme à la promesse d'un amour partagé.

Broken Sky est en ce sens la quête du couple parfait. Le film ouvre sur une citation du script de Marguerite Duras pour Hiroshima mon amour d'Alain Resnais (1959):

Cela viendra lorsque nous ne saurons plus quel nom donner à ce qui nous unit. Ce nom sera effacé petit à petit de nos mémoires. Et bientôt, il disparaitra complètement. ${ }^{6}$

Une scène faiblement éclairée au flash s'ouvre sur un corps mâle extatique, purement satisfait. Broken Sky file l'illusion du couple parfait, quasi céleste. Une voix off, intérieure et comme sans âge, insiste: "Je pense à toi le matin, l'après-midi et le soir..., toujours à toi...» On peut entendre le bruit d'un feu de cheminée. Cette séquence semble ainsi une préfiguration du concept de nation androgyne, lieu où le genre n'est plus le marqueur central et où l'égalité est donc possible. Plus encore: un lieu où l'amour serait le seul but de l'humanité. C'est là l'un des énoncés principaux du film de Hernandez. Les corps y sont moyens de communication. Quasi aucun mot n'est prononcé ni requis. Regards, caresses, travellings circulaires suffisent au récit qui semble se muer en une danse chorégraphiée. L'amour semble être une force essentiellement corporelle, une griserie. Confirmant l'intensité de ce qui est ressenti par les amants, Hernandez place des paroles didactiques du professeur de philosophie sur Aristophane et le mythe androgyne. Ce dernier intervient lorsque Gerardo se remémore l'extraordinaire puissance de la passion romantique qui l'unit à son amant. L'explication du professeur est réduite à sa plus simple expression, mais elle suffit à nous faire comprendre le principe philosophique de l'imagination de Hernandez dans ce film. Il y développe un langage

6. Hiroshima Mon Amour, New York, Grove Press, I96I, p. 77. 
et un récit cinématographiques quasi silencieux aux effets étonnants. On pense au monde d'ambiguïté et de contentement sexuels du poème de Swinburne Fragoletta:

O Love! What shall be said of thee?

The son of grief begot of joy?

Being sightless, wilt thou be

Maiden or boy?7

Une pulsion primaire provient du locus utopique de l'hermaphrodite et de l'androgynie, non pas comme perversion mais comme condition idéale d'une humanité en quête d'égalité. En termes socioculturels, cette pulsion est à l'œuvre dans certaines œuvres depuis le XIX ${ }^{\mathrm{c}}$ siècle, interrogeant sans relâche la structure morale de l'ordre capitaliste et patriarcal. Les protagonistes principaux de ce film sont mus par de tels idéaux. Ils ne se perçoivent pas eux-mêmes comme différents, anormaux ou marginaux. Ils ne souffrent pas d'être homosexuels, mais seulement de leur désir d'amour. Ils n'existent que pour eux-mêmes. Un corps misanthrope apparait ici, comme miroir du moi, miroir qui n'est là que pour refléter des subjectivités en mal de romance. La perversion, une fois de plus, est absente de ces corps. À la place, une perception fraîche et candide du monde est donnée à voir.

Cependant, dans cette sorte de bulle paradisiaque, le bizarre apparait. Le ciel est brisé lorsque Jonas trahit l'amour idéal, laissant un troisième homme s'immiscer et le recouvrir de sa passion. Cet «autre» fantasmatique occulte la complétude que les deux jeunes amants avait réussi à créer. Tout d'abord par un regard, puis par un placement des corps, l'ombre de cet autre amenant désolation plutôt que joie et bonheur. Ce lancinant et secret désir de Jonas pour l'autre corps est d'emblée visible pour Gerardo. Le film nous le montre dans des scènes où l'autre corps remplace celui de Gerardo de façon fantasmatique, caressé et embrassé en rêve. Mais la menace de l'étrange altérité est supprimée - réprimée par le happy end où le cinéaste choisit de représenter l'amour androgyne, l'ordre androgyne, comme rien d'autre qu'une utopie.

7. A. C. Swinburne, The Poems of Algernon Charles Swinburne, vol. I, Londres, Chatto \& Windus, I904. p. 82. Voir J. Simmons, «Algernon Charles Swinburne and the philosophy of androgyny, hermaphrodeity, and Victorian sexual mores ", The Victorian Web, Literature, History and Culture in the Age of Victoria, [en ligne], [URL: http://www. victorianweb.org/authors/swinburne/simmonsi2.html], consulté le Ir mars 20 IO. 


\section{Corps impossibles}

Reygadas est un cinéaste bien singulier. Après avoir étudié le droit à l'université, il décide de devenir réalisateur et reçoit un accueil international très favorable pour son premier film, Jàpon. Bien qu'il prétende ne pas faire de sociologie dans ses films, il y observe suffisamment de gestes de (dé-)codification des représentations sociales mexicaines pour que l'on puisse les étudier sous cet angle. La nation, le caractère religieux de la culture populaire mexicaine, les relations ethniques et de classes semblent être au centre d'un récit par ailleurs confus. Si nous pouvons considérer l'homosexualité - en tant que subversion de l'ordre de genre - comme étant au centre des significations de Broken Sky, dans Bataille dans le ciel, la disruption de l'ordre symbolique est rendue possible par un corps de classe ethniquement marqué. Minimaliste en termes de dialogues - ils sont peu fréquents et passablement étranges -, le film se fonde sur la puissance et l'impact du visuel. Ici, le contenu subversif vient de la représentation cinématographique de ce que l'on pourrait appeler les corps impossibles: des corps négligés, jamais visibles, inexistants sur grand écran. Des corps qui sont le contraire du modèle de beauté normative prévalant, et dont la représentation, lorsqu'ils se prêtent à des actes sexuels explicites, devient grotesque.

Déjà dans Jàpon, Reygadas avait eu recours à une scène sexuelle très commentée entre une vielle femme indigène et un homme entre deux âges. Cette volonté de représenter des corps d'ordinaire non représentés, ainsi que leurs relations, est encore plus forte dans Bataille dans le ciel. Des images choquantes structurées autour de la transgression de frontières raciales et de classes contiennent, dans leur esthétisme, le flux du récit. Cela se voit d'ailleurs à l'incapacité des critiques latino-américains de nommer ce qui a lieu, désignant Marcos comme un «métisse», ou «le gros chicanos », ou encore l'«indigène ». Mais qui est Marcos? Il est fait ici usage des corps comme d'un provoquant prétexte à la description d'un monde déséquilibré, un monde divisé par les classes sociales et le racisme.

Le réalisateur déclare que, en réalisant Bataille dans le ciel, il voulait:

[...] faire un film sur le conflit intérieur d'un homme, dans le contexte d'une ville et d'un certain milieu social. On pourrait considérer que le lieu du tournage fait un peu cliché ou piège à touristes, avec sa basilique, sa cathédrale, son métro, son stade... Hitchcock prétendait que pour filmer correctement les Pays-Bas, il fallait en filmer les clichés, comme les mou- 


\section{Le corps-récit}

lins à vent, les parapluies, etc. J'ai filmé Mexico de cette façon. Il y a du coup quelque chose de très fortement mexicain dans mon film, mais d'un autre côté, la situation en est très universelle et ouverte à interprétation. ${ }^{8}$

Le Mexique est en réalité représenté par des clichés qui finissent par devenir quelque chose d'autre du fait de leur extrême abstraction. Le drapeau mexicain, l'ambiance renfermée du métro et de ses couloirs, les foules de gens visiblement représentatifs, le pèlerinage à la basilique de la Guadalupe, principal site du culte populaire de la Vierge, façonnent un monde fait de lignes culturelles pour le moins marquées. Tout cela est relié à l'autre forme par laquelle le cinéaste dépeint le vrai monde mexicain: le vrai peuple, les vrais Mexicains. Mais faire figurer des corps réels fit polémique. La critique y vit une rupture bienvenue avec la façon hollywodienne de styliser les corps selon un idéal de perfection abstrait, mais aussi une grotesque provocation pénible à regarder.

Recherchant le point de tension maximale entre l'imaginaire cinématographique type et des effets de réel, fût-ce à travers ce qui peut être considéré comme une stratégie de la provocation visuelle ${ }^{9}$, Reygadas prétend qu'il s'était fait un principe de ne pas faire appel à des acteurs professionnels. Il ne voulait pas d'experts représentant quelqu'un précisément. Au contraire il s'était dit intéressé par l'énergie qu'irradient les «vraies gens». Il voulait rendre cette énergie signifiante et se montrait très critique des standards de beauté qui régissent la culture visuelle de masse, et encore plus de l'invisibilité, dans le cinéma mexicain, des «métisses», comme il les appelle, indiquant que $80 \%$ de la population mexicaine n'est ni blanche ni blonde, bien que $95 \%$ de l'imagerie culturelle le prétende en le montrant.

Une telle recherche de visibilité cinématographique des vraies gens relève d'une intention de déconstruire les modèles dominants de représentation du corps. De même, en montrant une relation sexuelle peu commune entre un homme non attirant et sa patronne, le but du réalisateur est d'exhiber les structures socioculturelles dominantes. Ce faisant, il instaure une transgression non seulement dans la relation sexuelle maître / serviteur ou patron/employé, mais aussi entre corps marqués ethniquement. Ces derniers sont cruciaux dans l'histoire. Ce sont des

8. La Jornada, Mexico, 17 septembre 2005. Les citations sont traduites par l'auteur.

9. "Érotisme n'est peut-être pas le bon mot concernant Bataille dans le ciel. Les scènes de sexe [...] échappent au réalisme fantasmagorique de la pornographie et au romantisme fantastique des scènes d'amour hollywodienne ", C. Campbell, "Review of Battle in Heaven», Cinematical, [en ligne], [URL: http://www.cinematical.com/2006/02/18/ review-battle-in-heaven], consulté le is avril 20 IO. 
corps parlants. Ils réalisent la subversion simplement en étant filmés, par la façon dont ils le sont. Le réalisateur nous met face à des gestes sans raison ni motif. La force de l'image est créée par les corps eux-mêmes et la façon dont ils sont présentés. Le drame est contenu dans le corps statique et ses gestes. Reygadas déclare:

Nous apprenons beaucoup via leurs corps [...]. Il y a toujours des masques; chacun est un acteur à un moment donné. Les corps sont objectifs, et c'est pourquoi je les filme longuement, même lorsqu'ils sont immobiles. Je trouve que les corps sont magnifiques dans ce film. ${ }^{10}$

Le film conte l'histoire de Marcos (Marcos Hernandez), le chauffeur d'un général, et de sa femme (Bertha Ruiz) dont on ne connait pas le nom, qui vend des montres et des bonbons dans le métro. Ils ont kidnappé l'enfant de leur voisin, qui vient à mourir accidentellement. Marcos, responsable de la levée des couleurs, chaque matin, sur la place centrale, est hanté par ce décès. Il se confesse à Ana (Anapola Mushkadiz), la fille du général, à qui il sert également de chauffeur. Ana se prostitue pour se divertir. Elle s'offre à Marcos à la fois pour son plaisir et pour le sien. À la fin du film, Marcos la tue puis participe au pèlerinage en l'honneur de la Vierge de Guadalupe dont lui a parlé sa femme, bien que nous sachions qu'il considère ces processions comme une ineptie. À la fin, totalement seul et désespéré, il s'en remet à la Vierge, mais aucune rédemption n'est plus possible pour lui.

Marcos et sa femme luttent pour survivre et éprouvent la dureté de l'existence, ce qui est communiqué par des regards échangés et par de courts dialogues. Ana, elle, est une riche, blonde et très belle jeune femme. Marcos travaille pour sa famille depuis de nombreuses années. À plusieurs moments nous pouvons sentir comment Marcos subit la discrimination raciale. Il est maltraité dans le métro et dans la rue lorsqu'il conduit. Ana est elle-même raciste dans la façon dont elle parle des employés de maison de Jaime, son petit ami. Mais envers Marcos, son attitude est ambiguë. Elle connaît et aime Marcos, en ce que l'amour, ici, signifie domination.

La toute première scène du film montre une fellation pratiquée sur Marcos par Ana, scène on ne peut plus explicite et pourtant non sexuelle. Il a été dit du film qu'il montrait des corps obèses et grotesques en train d'avoir des relations sexuelles, qu'il était un film érotique. Mais est-ce vrai-

IO. «El sexo entre cuerpos perfectos, farsa del cine comercial», La Jornada, Mexico, I7 mai 2005 . 
ment le cas? Reygadas filme-t-il vraiment des scènes sexuelles? Et dans la négative, que filme-t-il donc de la sorte? Que dit le film qui ne peut être mis en mots? Quelle est cette "objectivité » que Reygadas assigne aux corps? N'est-elle pas celle des marqueurs sociaux et symboliques?

Il n'y a rien d'explicite dans ce film, et encore moins de scènes de sexe explicites, même si elles sont directes et peu compliquées. La subtilité tiendrait plutôt à accoupler la «belle» Ana (jeune, mince, puissante et riche) et le «grotesque» Marcos (gros, indigène, de couleur et pauvre). Dans les termes de Juan Eduardo Murillo:

L'aspect fondamental de cette scène c'est le «et». Le gros chicanos «et» la jolie fille. Il y a quelque chose d'inhabituel et de pervers dans cette relation, quelque chose qui n'appartient à aucun des protagonistes, qui relève d'une image mentale, d'un échange, d'un cadeau entre eux, en un mot, l'Argent..."

L'argent est montré comme ce qui traverse toutes les relations contemporaines, comme le moteur véritable du désir, mais aussi de la domination et de la subordination. Pourtant le film ne fait jamais directement allusion au pouvoir de l'argent et à la corruption sociale. Selon Murillo, il se passe dans le film de Reygadas quelque chose d'analogue à ce que Deleuze a vu dans le Six fois deux de Jean-Luc Godard et Anne-Marie Miéville (réalisé en 1976 pour la télévision) : un certain bégaiement fait du film un étranger dans sa propre langue. Dans Bataille dans le ciel, «des Mexicains doivent être sous-titrés pour des Mexicains, lesquels deviennent étrangers à leur propre représentation, à leur propre image» (ibid.).

Le film comprend quatre scènes érotiques. La même, dédoublée, ouvre et ferme le film: c'est celle de la fellation. Toutefois, un changement peut être observé. La face silencieuse et hiératique de Marcos d'une part et les larmes d'Ana d'autre part n'apparaissent plus dans la dernière scène. À la place, on voit un Marcos souriant qui déclare son amour à Ana, et elle le recevant du regard. Ces scènes interdisent au spectateur tout point de vue pornographique: elles sont trop clairement, trop évidemment non naturelles. Leur répétition et le déplacement de signification induit par le changement d'expression des personnages ferment le cycle symbolique.

La seconde scène de sexe, celle entre Marcos et son épouse, empêche également le plaisir voyeuriste, non pas à cause des corps obèses, mais

I I. J. E. Murillo, «Batalla en el cielo. Recodos y tartamudeos», La Fuga. Revista de cine, [en ligne], [URL: http://lafuga.cl/batalla_en_el_cielo/I37], consulté le I5 avril 20 IO. 
parce qu'une représentation d'une peinture de la Vierge pleurant son fils mort dans le style de Giovanni Bellini est placée sur le mur dominant l'espace, convertissant l'acte sexuel en scène sacrificielle, non sans une certaine tendresse.

La séquence montrant Ana et Marcos en train de faire l'amour est un moyen pour le réalisateur de mettre en image ce que le récit ne dit pas: le gros corps de Marcos est statique au-dessous de celui, mouvant, d'Ana. Le plaisir se lit sur le visage comme dans l'attitude et les gestes de cette dernière. Lorsque Marcos commence à lui toucher les seins, elle lui dit d'arrêter, soulignant ainsi qu'elle se réserve la position active et dominante. La caméra quitte la pièce, nous laissant apercevoir la vie de tous les jours, puis revient. Un extraordinaire plan en plongée nous donne à voir l'image des corps d'Ana et de Marcos après l'amour. Ils demeurent dans une douce immobilité. Soudainement, par l'image comme par la musique qui semble funéraire, leurs corps se transforment en deux cadavres. La mort fait son apparition subtilement, la mort de l'être-ensemble de ces deux corps. Leurs sexes sont ensuite filmés en gros plan, comme s'ils fixaient la caméra ou lui parlaient. Reygadas produit ici l'une des images les plus signifiantes du film, par laquelle le spectateur se voit rappeler la présence réelle des corps symboliques.

Le récit proprement dit est secondaire pour Reygadas. Son objectif est de susciter une perception aiguë de lieux, culturels et émotionnels, ainsi que de l'épineuse dynamique de classe. Par un travail sur le son et de longs plans ininterrompus, Reygadas parvient à créer la sensation d'un monde existant hors cadre. À propos de la fameuse scène de fellation, il remarque qu'elle contient plus de mystère qu'elle ne déploie d'érotisme ou de pornographie, un mystère qui suscite la curiosité, celle-là même de Reygadas:

Ce n'est pas un film pornographique. Dans un film pornographique une fellation est là pour exciter le spectateur. Dans la scène d'ouverture de mon film, on voit le visage d'un homme, son corps, puis la fille qui regarde vers la caméra, en pleurant. Ce que je voulais, c'était produire une image qui va au-delà d'un simple acte sexuel. Ce n'est pas quelque chose de naturel, mais plutôt, en tant qu'observateur, une sorte de mystère. ${ }^{12}$

Mais même si nous acceptons ce mystère comme ce qui appelle et dirige le regard du film lui-même, n'en demeure pas moins la problématique question du corps comme objectivité. Qu'est-ce qui transparait si direc-

I2. J.-J. Olivares, «Entrevista a Carlos Reygadas», La Jornada, 8 octobre 2005. 
tement et en même temps mystérieusement de ces corps? Marcos luimême n'est-il pas porteur du mystère pour le spectateur? La façon dont Reygadas montre ces «vrais» corps dans leurs «vraies» vies n'est-elle pas, en fin de compte, vide d'une "réelle» humanité? Le film peut-il porter un regard sur les pauvres et les dominés qui ne soit pas animé d'un désir de sublimation ou de dédain, et qui ainsi les rende humains? Reygadas tente-t-il d'agencer une métaphore critique de la culture raciale mexicaine en se servant des corps? Ou finit-il par réinscrire la «mexicanité» dans la vision racialiste qu'il essaye précisément de critiquer?

La caméra suit le regard de Marcos, nous faisant partager son isolement. Mais elle suit également Marcos comme corps objectif. Cette ambiguîté demeure toujours à l'état de tension irrésolue entre deux forces à l'œuvre dans l'image: tantôt la caméra demeure à distance de Marcos, l'observant comme s'il était un insecte, de manière entomologiste; tantôt elle devient subjective et nous présente le monde depuis son point de vue, tel un flou, un mystère, qui revient pour nous atteindre. ${ }^{13}$ Cette ambivalence produit une relation lâche aux personnages et rend le regard du film distant en favorisant l'observation de l'autre comme anormal, plutôt qu'une observation avec ou depuis l'autre. Reygadas ne perpétue-t-il pas alors une construction exotique - et érotique - de l'autre telle qu'elle apparaît dans certaines critiques du film:

Mais le pays que célèbre le festival n'est pas celui des sombreros et de la baie d'Acapulco. C'est le Mexique immémorial de la cruauté et du sang, de la célébration du sexe et du sacrifice de la chair humaine, de la collusion carnavalesque entre la vie et la mort. Le Mexique des dieux de la religion et des dieux du néolibéralisme, qui se disputent, sous l'ombre sacrificielle du Crucifié, le privilège de la férocité. Le Mexique tel que Ripstein, Buñuel et Eisenstein l'ont filmé, et tel que ce dernier l'a défini au plan à la fois esthétique et moral: "Dans ses deux aspects, espagnol et aztèque, la simplicité du grandiose y côtoie l'impétuosité du baroque. »14

Le film de Reygadas n'affirme-t-il pas, au lieu de la déconstruire, la politique des corps hégémoniques? Maintenir des fractures internes et des divisions sociales comme une doxa naturalisée, tout en la rendant "mystérieuse ", n'est-ce pas reproduire l'incompréhensible altérité? Les réponses sont multiples et variées, et le film est suffisamment ambigu et

I3. Voir M. Dargis, «Displaying a keen eye even for the repellent», The New Tork Times, I7 février 2006.

I4. J. Mandelbaum, dans Le Monde, I7 mai 2005. 
provocant pour soutenir des interprétations radicalement différentes. Il a été censuré pour le public mexicain, les scènes de sexe étant floutées - ce qui n'est qu'un exemple de l'effet attendu des corps cinématographiques sur le public. Mais tandis que le cinéma, loin de rester confiné dans un univers académique, se déploie dans le «vrai monde» et ses enjeux politiques, nous nous devons de mentionner un autre aspect de ce film. Bertha Ruiz, la femme qui joue l'épouse de Marcos, déclare:

Cette expérience a changé ma vie parce que j’y ai réussi ce que je n'avais jamais réussi avant: m'accepter telle que je suis. Lorsque je me suis vue nue en face de la caméra, j'ai compris que je ne montrais pas mon corps mais mon âme. ${ }^{\mathrm{Is}}$

Paradoxalement, le corps nu, dans sa matérialité, transfiguré par son devenir cinématographique, est devenu pour elle le chemin vers son corps intérieur, cet étrange dedans qui nous rend si peu naturels.

D’une certaine façon, le cinéma propose toujours des corps impossibles, si beaux, tellement plus désirables que nos corps réels. Mais bien que ces corps hyper-désirables et improbables soient la norme dans la plupart des films, il est d'autres corps impossibles: ceux qui ne peuvent être représentés, parce qu'ils n'apparaissent jamais sur Cellulö̈d. Ainsi avons-nous parlé ici de ceux qui sont irreprésentables du fait de l'hétéronormativité sexuelle ou de la normativité de classe ou encore de race. En insistant sur ces films dans le cinéma mexicain contemporain, nous avons suggéré que les corps au cinéma sont les signifiants d'un ordre symbolique, mais aussi le site de possibles transgressions de cet ordre.

Les corps ne sont jamais que des corps à l'écran. Ils constituent le site où les relations socioculturelles et symboliques sont représentées et contestées. La présence et la représentation du bizarre prennent diverses formes, dans divers contextes. Dans Broken Sky, une nation androgyne idyllique est préfigurée comme le souhait utopique de supprimer le bizarre ou l'étrange. Dans Bataille dans le ciel, ce dernier est l'apparence corporelle que prennent les corps des classes et des races négligées. La sexualité est un puissant moyen de décrire la menace tapie dans l'altérité raciale et culturelle.

15. La Jornada, 23 octobre 2005. 


\section{Table}

Introduction. Corps-cinéma

Jérôme Game

\section{FLUX}

Des corps effacés par le flux: Hou Hsiao-hsien, Ozu et Wong Kar-wai

Vincent Amiel

D’un corps chez Yoshida Kijū. Le cinéma: du mouvement au déclenchement

Jean-Michel Durafour

La forme et l'informe: de la dissolution du corps à l'écran Martine Beugnet

\section{VIRTUEL}

L'image ralentie: de la caméra-œil au bullet-time Elie During

Entre cinéma et photographie: quelques variations de vitesse de l'image contemporaine 


\section{RÉCITS}

Du visage au dos: en relisant Levinas avec les frères Dardenne Sarah Cooper

Le territoire de l'extase : le corps et le paysage dans l'œuvre de Bruno Dumont

Ludovic Cortade

Images sans organes / récit sans télos: Carlos Reygadas

et Gus Van Sant

Jérôme Game

\section{POLITIQUES}

Insurrections de l'amour en contexte matérialiste :

Garrel, Costa, Waked

Nicole Brenez

Des corps sans visages: ce que le cinéma fait avec les foules

Pierre Sorlin

Vérité du désir, vulnérabilité des corps: Boys Don't Cry

Emma Wilson

Le corps-récit: Julian Hernandez et Carlos Reygadas 


\section{Titres récents, dans la même collection}

Jean Tardieu.

Des livres et des voix

Sous la direction de Jean-Yves Debreuille

2010

Langston Hughes.

Poète jazz, poète blues

Frédéric Sylvanise

2009

Apprendre à porter sa vue au loin.

Hommage à Michèle Duchet

Textes réunis par Sylviane Albertan-Coppola

2009

Rêver d'Orient, connaître l'Orient

Sous la direction d'Isabelle Gadoin

et Marie-Élise Palmier-Chatelain

2008

Bernard Noël: le corps du verbe

Sous la direction de Fabio Scotto

2008

Poésie vacante.

Nerval, Mallarmé, Laforgue

Sous la direction de Henri Scepi 2008

Ceci n'est pas une tragédie.

L'écriture de David Markson

Sous la direction de Françoise Palleau-Papin

2007

De Drake à Chatwin:

rhétoriques de la découverte

Sous la direction de Frédéric Regard

2007
Denis Roche:

l'un écrit, l'autre photographie

Sous la direction de Luigi Magno 2007

La lumière noire d'Elsa Triolet

Alain Trouvé 2006

Gilbert \& George / El

Sous la direction d'Isabelle Baudino et Marie Gautheron 2005

Aragon, Le Fou d'Elsa:

un poème à thèses

Hervé Bismuth

2004

Balzac, suite et fin

Franc Schuerewegen

2004

Euvres majeures, œuvres mineures?

Sousladirection deCatherineVolpilhac-Auger

2004

Ponge, résolument

sous la direction de Jean-Marie Gleize

2004

L'Orient des femmes

Sous la direction de

Marie-Élise Palmier-Chatelain

et Pauline Lavagne d'Ortigue

2002 
Cet ouvrage, composé en Galliard, a été mis en page par les soins du service des éditions de l'École normale supérieure de Lyon.

Il a été reproduit sur papier Olin naturel.

Dépôt légal : juillet 2010

Réimpression en janvier 2013 par l'imprimerie Graphiscann Numéro d'imprimeur :

IMPRIMÉ EN FRANCE 